$1 / 8^{\circ}$ FINAL REPORT

LBL-8616 GSRMP-2 UC-37

\title{
INSTRUMENTS FOR SUBSURFACE MONITORING OF GEOTHERMAL SUBSIDENCE
}

\section{MASTER}

\author{
J. E. O'Rourke and B. B. Ranson \\ Woodward-Clyde Consultants
}

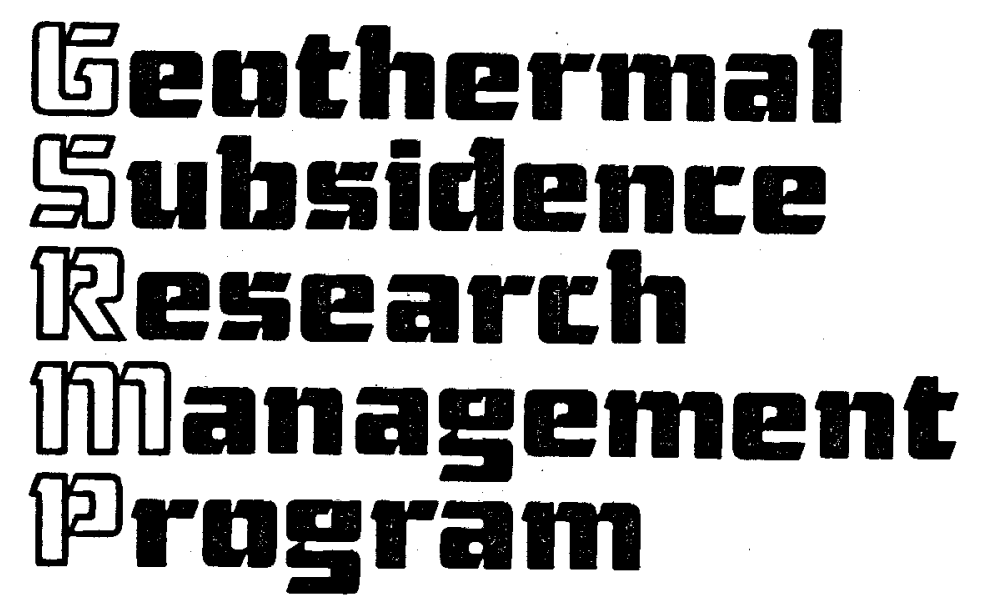

July 1979

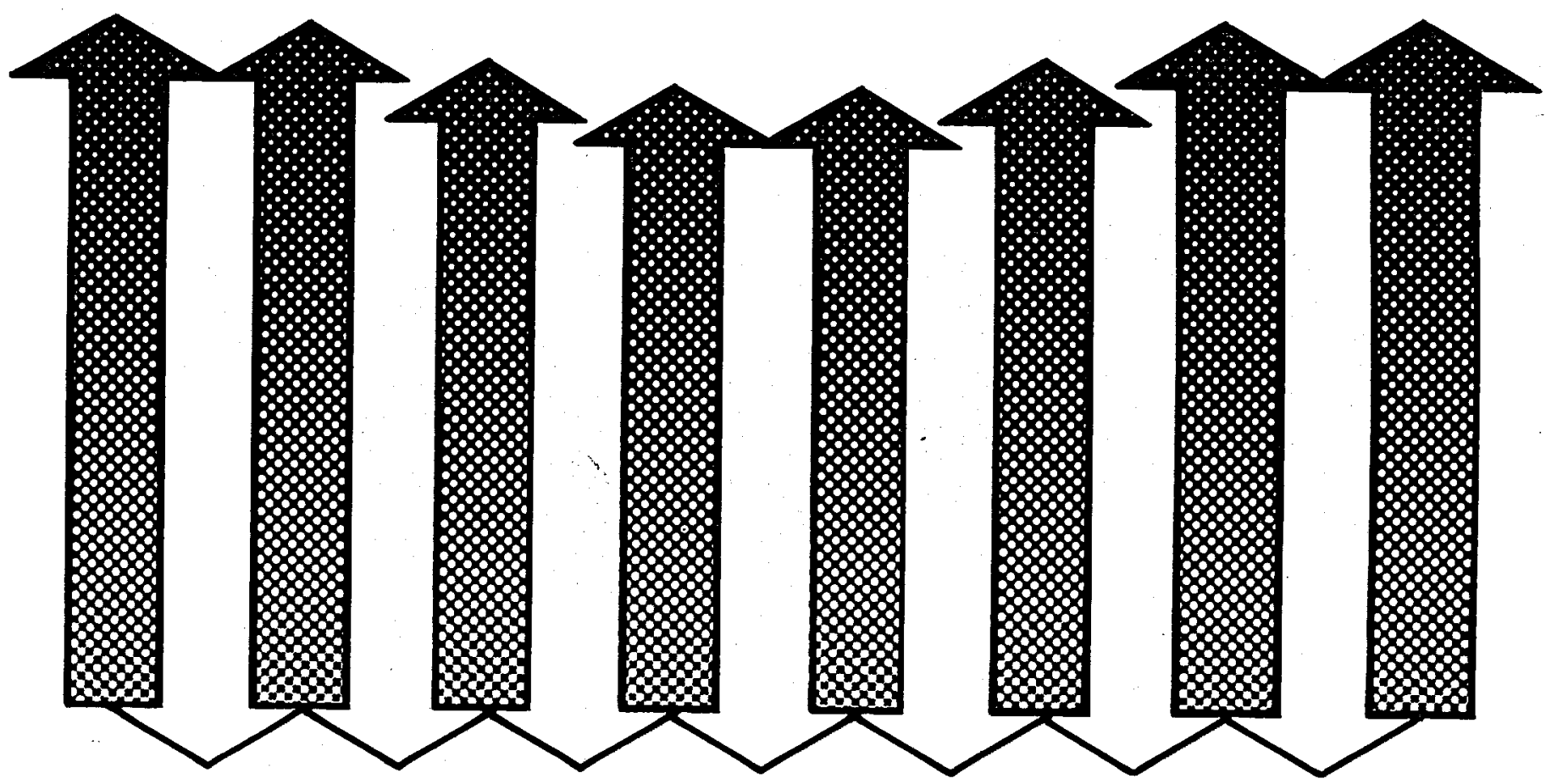

Earth Sciences Division

Lawrence Berkeley Laboratory

University of California

DISTRIEUTION OF THIS OSCUBRENT IS UHLINATITEO

Prepared for the U.S. Department of Energy under Contract W-7405-ENG-48 
This book was prepared as an account of work sponsored by an agency of the United States Government. Neither the United States Government nor any agency thereof, nor any of their employees, makes any warranty, express or implied, or assumes any legal liability or responsibility for the accuracy, completeness, or usefulness of any information, apparatus, product, or process disclosed, or represents that its use would not infringe privately owned rights. Reference herein to any specific commercial product, process, or service by trade name, trademark, manufacturer, or otherwise, does not necessarily constitute or imply its endorsement, recommendation, or favoring by the United States Government or any agency thereof. The views and opinions of authors expressed herein do not necessarily state or reflect those of the United States Government or any agency thereof.

\author{
Printed in the United States of America \\ Available from \\ National Technical Information Service \\ U.S. Department of Commerce \\ 5285 Port Royal Road \\ Springfield, VA 22161 \\ Price Code: A07
}




\section{DISCLAIMER}

This report was prepared as an account of work sponsored by an agency of the United States Government. Neither the United States Government nor any agency Thereof, nor any of their employees, makes any warranty, express or implied, or assumes any legal liability or responsibility for the accuracy, completeness, or usefulness of any information, apparatus, product, or process disclosed, or represents that its use would not infringe privately owned rights. Reference herein to any specific commercial product, process, or service by trade name, trademark, manufacturer, or otherwise does not necessarily constitute or imply its endorsement, recommendation, or favoring by the United States Government or any agency thereof. The views and opinions of authors expressed herein do not necessarily state or reflect those of the United States Government or any agency thereof. 


\section{DISCLAIMER}

Portions of this document may be illegible in electronic image products. Images are produced from the best available original document. 


\section{INSTRUMENTS FOR SUBSURFACE MONITORING OF GEOTHERMAL SUBSIDENCE}

This book was prepared as an account of work sponsored by an agency of the United States Gowemment. Neither the United States Government nor any soncy thereof, nor any of their omployees, makes any Cor

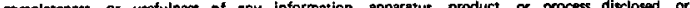
represents the its use would not intringe privately oumed rights. Referenos herein to any specific y.

not necesserily constiture or imply its endorsement, recommendation, of favoring by the United

States Government or any agency thereof. The views and opinions of authors expressed herein do not

necessarily state or reflect those of the United Stotes Government or any mency thereot.
Prepared for

Lawrence Berkeley Laboratory

University of California Berkeley, California 94720 under P. O. 2663402

By

J. E. O'Rourke and B. B. Ranson Woodward-Clyde Consultants Three Embarcadero Center San Francisco, California

July, 1979 
Reference to specific brands, equipment, or trade names in this report is made to facilitate understanding and does not imply endorsement by Lawrence Berkeley Laboratory.

The views and conclusions contained in this document are those of the authors and should not be interpreted as necessarily representing the official policies or recommendations of the Department of Energy or of the U.S. Government. 
This report was prepared by Woodward-Clyde Consultants, Western Region, San Francisco, under Lawrence Berkeley Laboratories (LBL) Purchase Order No. 2663702 and includes resul ts of research done between August 1977 and July, 1979. The subject research is pursuant to the LBL contract with the U. S. Department of Energy, Division of Geothermal Energy, for Geothermal Subsidence Research. It was administered under the technical direction of Messrs. Terry Simkin, John Noble and Dr. Werner Schwarz of LBL. Mr. H. A. Todd was the contract administrator for LBL.

The authors wish to acknowledge the program direction and constructive review of this study by Dr. Uirich Luscher, Woodward-Clyde Consultants' Principal-in-Charge for this project. We also wish to thank Ms. Mary Gillam, Mr. Robert L. Ray, Mr. Edward Fischer and Dr. Jerome Singer for their technical ass istance and review in their respective disciplines: geothermal subsidence processes, metaliurgy, insulation and refrigeration, and electrical engineering. We are also gra teful to Dr. R. L. McNeill and Mr. J. H. Wilson for their reviews.

This report and the four preceding interim reports greatly benefited from the yeoman assistance of Bayani DeLeon and Dante Tolosa, draftsmen and Anita Davy, Jackie Georges, Rodger Pitcairn and Lisa Rigler, technical typists.

This work was supported by the U. S. Department of Energy under Contract No. W-7405-ENG-48. 


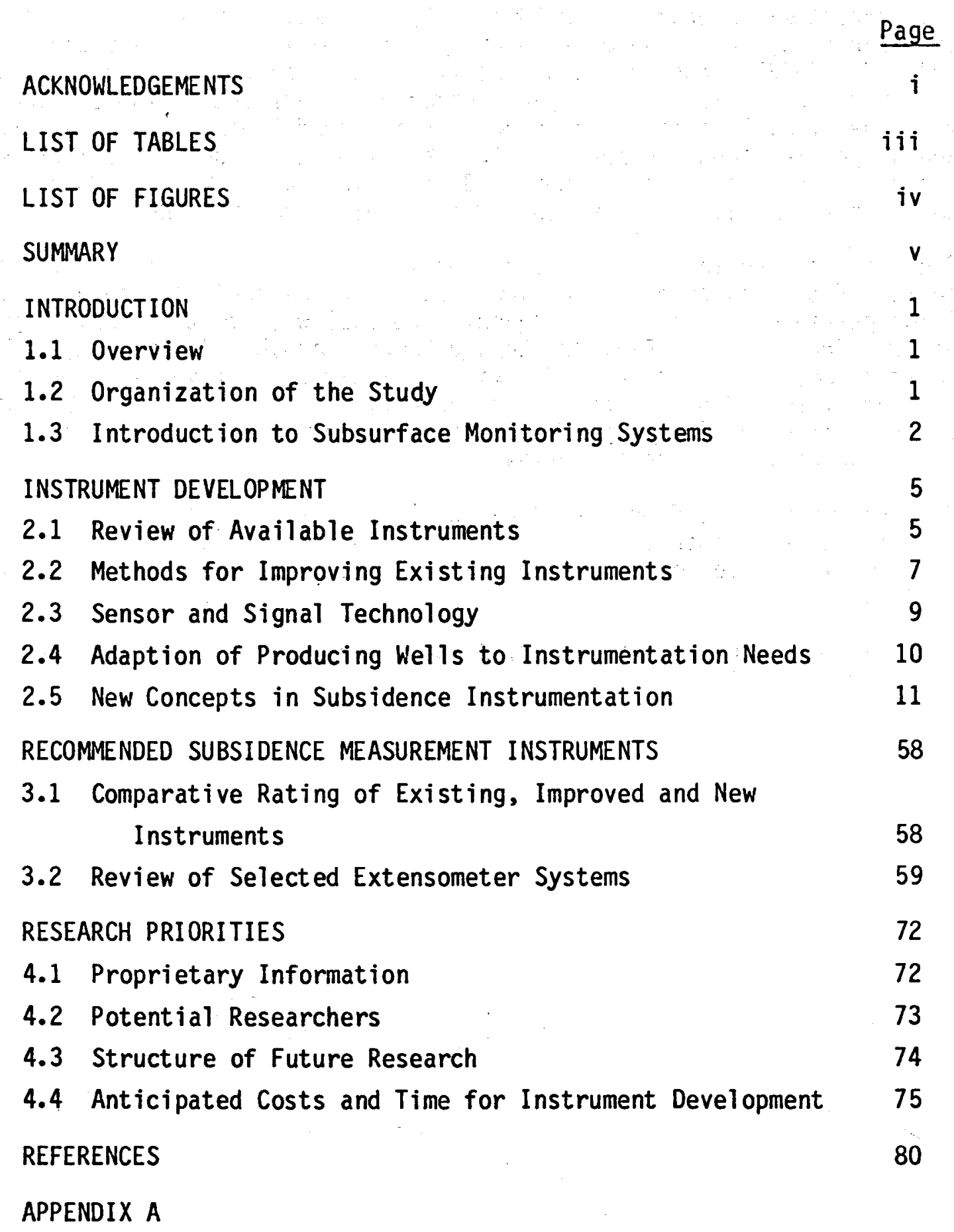

2.0 INSTRUMENT DEVELOPMENT

2.1 Review of Available Instruments 7

2.3 Sensor and Signal Technology 9

2.4 Adaption of Producing Wells to Instrumentation Needs 10

3.0 RECOMMENDED SUBSIDENCE MEASUREMENT INSTRUMENTS 58

3.1 Comparative Rating of Existing, Improved and New Instruments $\quad 58$

1.0 INTRODUCTION $\quad 1$

1.1 Overview 1

1.2 Organization of the Study 1 
TABLE 2.1 - COMPARISON OF GEOTHERMAL SUBSIDENCE AREAS 13

TABLE 2.2 - COMPARISON OF GROUNDWATER SUBSIDENCE AREAS 14

TABLE 2.3 - COMPARISON OF MAJOR OIL AND GAS SUBSIDENCE AREAS $\quad 15$

TABLE 2.4 - REPRESENTATIVE GEOTHERMAL RESERVOIR TEMPERATURES 16

TABLE 2.5 - PRODUCTION-INDUCED CHANGES IN THE AVERAGE TEMPERATURE OF GEOTHERMAL RESERVOIRS 17

TABLE 2.6 - GEOTHERMAL RESERVOIR PRESSURE CHANGES 18

TABLE 2.7 - CHEMICAL COMPOSITION OF GEOTHERMAL WATERS, in $\mathrm{mg} / 1 \quad 19$

TABLE 2.8 - SPECIFICATIONS FOR MONITORING SUBSURFACE VERTICAL DISPLACEMENTS 20

TABLE 2.9 - SPECIFICATIONS FOR MONITORING SUBSURFACE HORIZONTAL DISPLACEMENTS

TABLE 2.10 - RELATION BETWEEN SUBSIDENCE PARAMETERS AND WELL CASING PROTRUSION

TABLE 2.11 - CAPABILITIES OF EXISTING SUBSIDENCE MONITORING INSTRUMENTS

TABLE 2.12 - PROMISING INSTRUMENTS FOR GEOTHERMAL SUBSIDENCE MONITORING

TABLE 2.13 - INSULATION SUITABLE FOR GEOTHERMAL TEMPERATURES 28

TABLE 2.14 - THERMAL EXPANSION CHARACTERISTICS OF SELECTED MATERIALS

TABLE 2.15 - EFFECT OF TEMPERATURE ON ELASTIC MODULUS AND ELECTRICAL RESISTANCE

TABLE 2.16 - TEMPERATURE LIMITS OF MECHANICAL SEALS

TABLE 2.17 - SOME RECOMMENDED MATERIALS AND TECHNIQUES FOR GEOTHERMAL INSTRUMENTATION

TABLE 2.18 - PROMISING IMPROVED INSTRUMENTS

TABLE 2.19 - TRANSDUCER APPLICATION FOR GEOTHERMAL SUBSIDENCE 34

TABLE 2.20 - PERFORMANCE OF CIRCUIT ELEMENTS AT $300^{\circ} \mathrm{C} \quad 36$

TABLE 2.21 - SELECTED RECORDER SYSTEMS 38

TABLE 3.1 - MODIFIED RATING SYSTEM $\quad 65$

TABLE 3.2 - INSTRUMENT SYSTEM RATING MATRIX 66

TABLE 3.3 - GENERAL SPECIFICATIONS FOR SELECTED INSTRUMENT SYSTEMS 
Page

Figure 1.1 - PROGRAM FOR INSTRUMENT DEVELOPMENT (CATEGORY 3) 3

Figure 1.2 - THREE BASIC TYPES OF EXTENSOMETERS 4

Figure 2.1 - BASIC ELEMENTS OF POSSIBLE MECHANICAL HEAT PUMP 39

Figure 2.2 - CASCADING 3-STAGE THERMOELECTRIC REFRIGERATOR 40

Figure 2.3 - RELATION BETWEEN LENGTH INCREASE AND TEMPERATURE FOR CARBON STEEL AND INVAR

Figure 2.4 - USE OF THREE SENSOR PROBE TO DETERMINE DISTANCE BETWEEN DOWNHOLE MARKERS

42

Figure 2.5 - REED SWITCH SENSOR $\quad 43$

Figure 2.6 - SOME POSSIBLE SIGNAL CONDITIONING SYSTEMS

Figure 2.7 - A POSSIBLE ABOVE-GROUND COMPUTER STATION 45

Figure 2.8 - SLIP COUPLINGS FOR SUBSIDENCE MONITORING 46

Figure 2.9 - POSSIBLE CORRUGATION ARRANGEMENTS 48

Figure 2.10 - ACCESS GLAND FOR SETTLEMENT PROBE 49

Figure 2.11 - AUXILIARY CASING INSTALLATION 50

Figure 2.12 - MULTI-SENSOR REED SWITCH PROBE 51

Figure 2.13 - AUXILIARY MAGNA-CASING FOR REED SWITCH PROBE $\quad 52$

Figure 2.14 - VIBRATING WIRE METHOD 53

Figure 2.15 - PROXIMITY SENSOR PROBE METHOD $\quad 54$

Figure 2.16 - WEIGAND EFFECT SETTLEMENT DEVICE $\quad 55$

Figure 2.17 - DOUBLE FLUID SETTLEMENT DEVICE METHOD 56

Figure 3.1 - TRIPLE SENSOR INDUCTION PROBE METHOD 68

Figure 3.2 - TRIPLE SENSOR REED SWITCH PROBE METHOD 69

Figure 3.3 - TRIPLE SENSOR OSCILLATOR-TYPE MAGNET DETECTOR METHOD $\quad 70$

Figure 3.4 - TRIPLE SENSOR GAMMA RAY DETECTOR METHOD 71

Figure 4.1 - RECOMMENDED MANAGEMENT STRUCTURE FOR SUBSIDENCE
TOOL RESEARCH

Figure 4.2 - ALTERNATE MANAGEMENT STRUCTURE FOR SUBSIDENCE
TOOL RESEARCH

Figure 4.3 - OUTLINE OF PROPOSED GEOTHERMAL SUBSIDENCE RESEARCH (CATEGORY 3) 


\section{SUMMARY}

This report summarizes research carried out at Woodward-Clyde Consultants between September 1977 and May 1979. In this study the requirements for a subsurface geothermal subsidence instrument were reviewed. Available instruments for monitoring subsurface displacements, both vertical and horizontal, were studied and the most capable instruments identified. Techniques and materials for improving existing or developing new instruments were evaluated. Elements of sensor and signal technology with potential for high temperature monitoring of subsidence were identified. Drawing from these studies, methods to adapt production wells for monitoring were proposed and several new instrumentation systems were conceptually designed. Finally, four instrumentation systems were selected for future development.

These systems are:

- TRIPLE SENSOR INDUCTION SENSOR PROBE (with casing collar markers)

- TRIPLE SENSOR GAMMA RAY DETECTOR PROBE (with radioactive markers)

- TRIPLE SENSOR REED SWITCH PROBE (with magnet markers)

- TRIPLE SENSUR OSCILLATOR-TYPE MAGNET DETECTOR PROBE (with magnet markers)

All are designed for use in well casing incorporating slip couplings or bellows sections, although the gamma ray detector probe may al so be used in unlined holes. These systems all measure vertical movement. Instruments to measure horizontal displacement due to geothermal subsidence were studied and the required instrument performance was judged to be beyond the state-of-the-art. Thus, no conceptual designs for instruments to monitor horizontal movement are included in this report.

It is proposed that these four systems be laboratory tested to identify the most promising system. A full scale prototype of that system may then be constructed and field tested. Expected cost of this development is $\$ 1,040,000$ over a 3 year period. 
1.0 INTRODUCTION 


\subsection{INTRODUCTION}

The measurement of subsidence in geothermal areas can contribute to an understanding of the processes occurring in a geothermal reservoir area during production. Subsidence data can be used to build mathematical models, to assess the value of various production schemes such as water injection, and to predict future movements that may affect land use and particularly that might endanger wells and plant equipment. This study is concerned with identifying capable available instruments or recommending and specifying developmental work necessary for new instruments to monitor subsurface movements due to geothermal production.

The geothermal environment is hostile, and conventional equipment used for oil, gas and groundwater wells will not function in it. The challenge of this project was to develop a subsidence monitoring instrument that will survive the geothermal environment and that will provide accurate results. In the $300^{\circ}$ hot saline water of geothermal wells, rubber and plastics disintegrate, electronic components fail, corrosion and abrasion attack all but the most resistant steels, and severe scaling fouls moving parts. Conventional techniques have been carried to the limit for use in hot oil wells. In general they cannot be pushed further for geothermal use. Thus, off-the-shelf instruments do not exist for geothermal use. However, as we indicate throughout this report, technology in high temperature electronics and high-temperature corrosion-resistant materials is rapidly improving as a result of research on jet engine components, space vehicles, geothermal instrumentation, microwave transmission, and other areas. We have surveyed this emerging technology and have proposed four subsidence-monitoring instrument systems that incorporate many of these new developments and that we recommend for geothermal subsidence monitoring needs. The instruments and the development process are discussed below.

\subsection{OVERVIEW}

This report surmarizes a research project carried out at Woodward-Clyde Consultants from September 1977 to May 1979. It is part of a comprehensive study of geothermal subsidence, sponsored by the Department of Energy (D.0.E.) and administered by Lawrence Berkeley Laboratory. The D.0.E. project encompasses many aspects of geothermal subsidence, such as a review of case histories and an analysis of the economic impact of subsidence. The purpose of Category 3 of the overall LBL study is to develop a capability to measure vertical subsidence between two points in a geologic formation. The resulting instrument system must withstand the geothermal environment and must provide accurate data. This study of subsurface monitoring is the first part of a three-part project, as shown in Figure 1.1. The report represents the completion of Part 1, Conceptual Designs for Subsurface Monitoring of Geothermal Subsidence. The decision to undertake Parts 2 and 3 has not yet been made. A panel of industry representatives, reservoir engineers, and geothermal experts met in October 1978 to assess the feasibility of the proposed instrument development and to advise the D.O.E. Whether the probability of geothermal subsidence and associated problems was high enough to justify anticipated devel opment costs. Feedback from that workshop has been incorporated into the text of this report and is presented in Appendix $A$. 
This report provides a brief summary of research reported in four previous reports. It also contains a synthesis of that material, and concludes with recommendations for future research. The four interim reports, references WCC (1977a), WCC (1978a), WCC (1978b) and WCC (1978C), are included in this report as appendices, bound separately as Volume 2.

\subsection{ORGANIZATION OF THE STUDY}

The study of instruments for subsurface monitoring of geothermal subsidence began with a concerted effort to define the problem and measurement goals. The study then proceeded with a review of available instruments (WCC, 1977a), an examination of methods and materials that could be used to improve existing instruments or devel op new ones (WCC, 1978a), a review of sensor and signal technology to determine what instrumentation might be useful for new instruments or to modify existing ones (WCC, 1978D), and finally, the conceptualization of several new instruments which incorporate some of the favorable features of the technology studied and may be successful for geothermal work (WCC, 1978C).

All of these studies are brought together in this final report (Section 2). The candidate instruments are comparatively rated, and the most promising instruments are identified for future development (Section 3). Research needs and priorities for development of the instruments are discussed in Section 4.

\subsection{INTRODUCTION TO SUBSURFACE MONITORING SYSTEMS}

This report discusses instrumentation systems that may be used to monitor ground movements in and above geothermal reservoirs. The basic components of instrumentation systems that monitor vertical displacements (extensometers) and of those that detect horizontal movement (inclinometers) include (1) a Dorehole, (2) markers, (3) a sensing device and read-out, (4) a logging system, and (5) an orientation device (inclinometers only). The perfomance of each component of the instrument system will affect the quality of the data, as discussed in the report and in Appendix $A$.

Sketches of three common types of extensometers are shown in Figure 1.2 . Wire- and rod-type extensometers compare the known length of the wire or rod to the vertical distance between the downhole anchor and the surface. One type of probe extensometer measures the distance between a downhole marker and the surface using a distance-graduated cable. Another type compares the probe length to the spacing between adjacent downhole markers.

Inclinometers are usually probes. The angle of tilt of the probe is measured at successive depths. This value is then converted by integration to horizontal offset. Movements due to subsidence are determined for both extensometers and inclinometers by comparing data taken at different times.

The major portion of this report concerns extensometer development. Inclinometers were not studied after Interim Report 1 in view of the greater need for vertical subsidence monitoring instruments, and because we concluded that the technology needed to adapt inclinometer monitoring schemes to geothermal environments was beyond the present state-of-theart. 


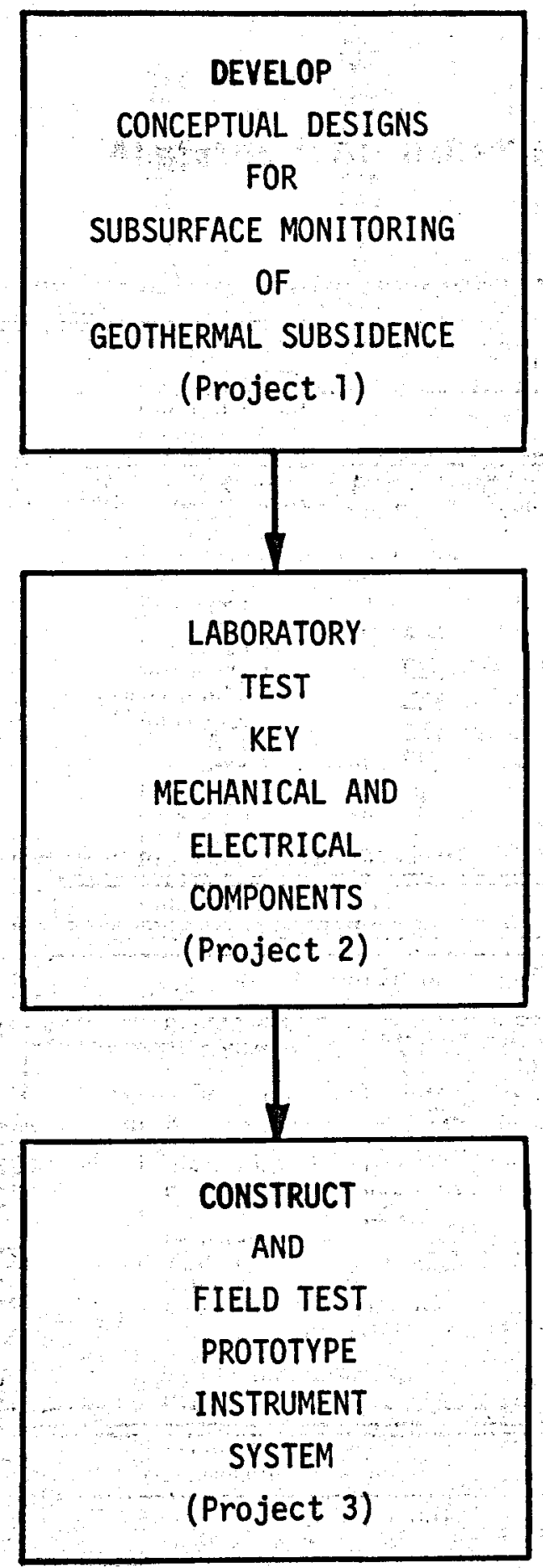

Figure 1.1 - PROGRAM FOR INSTRUMENT DEVELOPMENT (CATEGORY 3) 


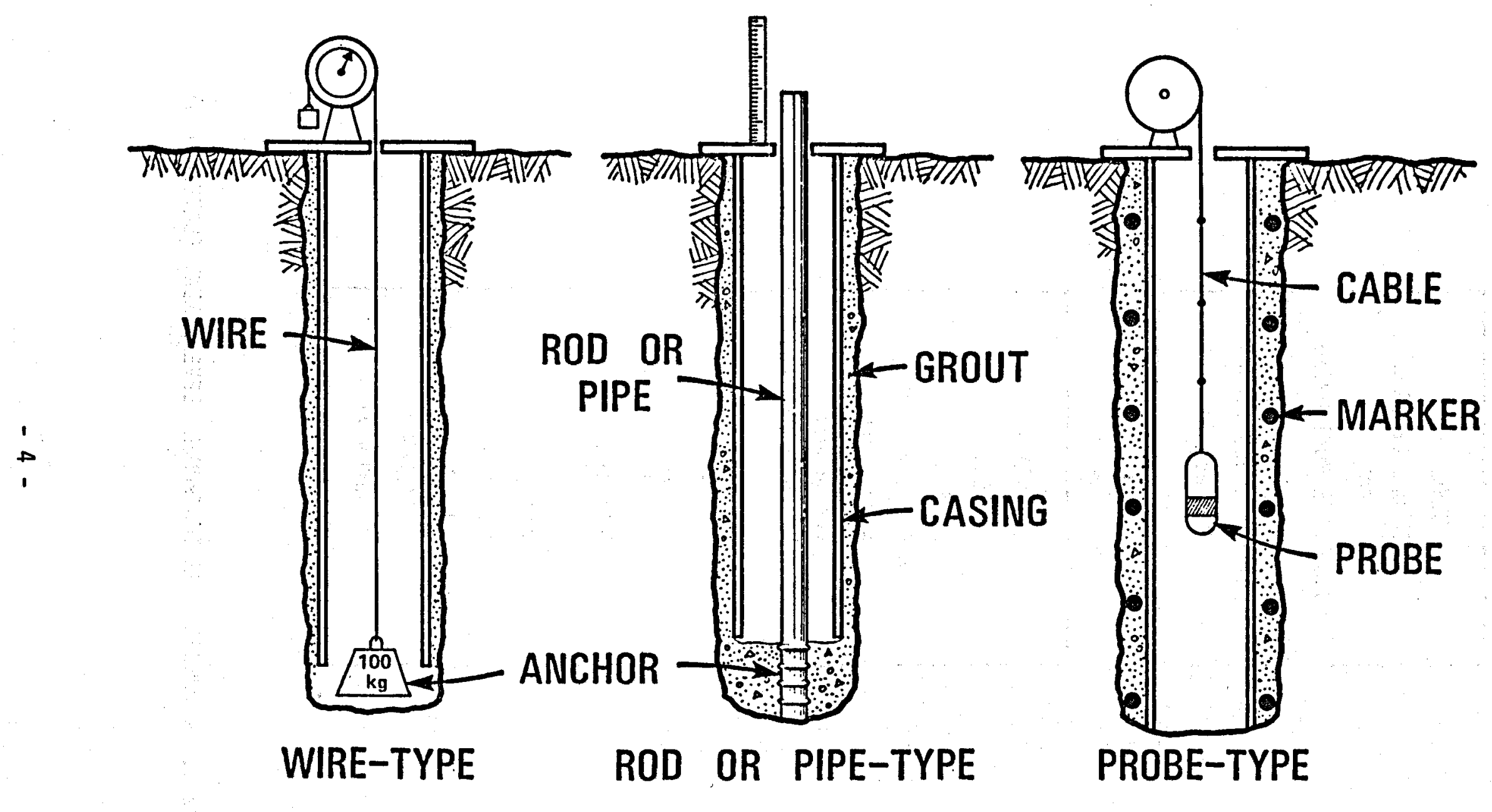

Figure 1.2 - THREE BASIC TYPES OF EXTENSOMETERS 
Woodward.Clyde Consultants

2.0 INSTRUMENT DEVELOPMENT 


\subsection{INSTRUMENT DEVELOPMENT}

This section provides an overview of the various studies that made up this project.

\subsection{REVIEW OF AVAILABLE INSTRUMENTS}

\subsubsection{Measurement Requirements}

The first step in this study was to define what the instrument must do and what hazards it must cope with (WCC, 1977a). He began by considering what measurements the instrument must make, the probable magnitude of those movements and what accuracy, monitoring frequency, and spacing between points would be useful to hydrologists, modelers, and civil engineers. Our review drew on avallable information on geothermal fields and production techniques (Table 2.1), supplemented by subsidence data from water (Table 2.2) and hydrocarbon (Table 2.3) withdrawal areas. We al so examined the special environmental problems, particularly high temperature (Tables 2.4 and 2.5), high pressure (Table 2.6), and corrosive, scaling fluids (Table 2.7 ), that a geothermal environment presents. The requirements imposed by the environmental problems and measurement needs were summarized in a set of specifications, which can be used to evaluate existing, modified or new instruments for subsidence monitoring; refer to Tables 2.8 and 2.9 .

Reinjection of waste brine will probably be practiced in most geothermal areas, with the benefit that the magnitude of subsidence is minimized. Consequentiy, the specifications in Tables 2.8 and 2.9 may in fact be minimum standards if reservoir engineers are to derive useful data on compaction processes from the field measurements (refer to Appendix A-3).

\subsubsection{Role of the Borehole}

To date, all reported subsurface strain measurements have been made in cased wells. We first evaluated whether a borehole is necessary at all. Gravity measurements appear to be the only geophysical method that can detect compaction at depth without a borehole. Unfortunately, this method is not likely to be useful because the neeeded input on fluid witndrawal and reservoir dimensions are hard to determine. Also while this method may provide average compaction, it does not provide a subsidence profile with depth (WCC, 1977a).

For measurement in cased wells, the effect of the casing on the local subisdence pattern and its measurement was examined. There are three basic problems. (1) Slip may occur between the ground and the well so that the commonly used technique of monitoring casing movement may not be meaningful. Slip, as indicated by well protrusion from the ground, has been noted in Mexico City, Mexico; the Po Delta, Italy; the Santa Clara Valley, California; and other fluid withdrawal areas (Table 2.10).

(2) Where a good bond exists between the casing and ground, the casing 
will draw loads onto itself in proportion to its modulus and may locally alter the settlement pattern. (3) In areas of large subsidence movements, a good casing/ground bond may result in compression large enough to fail the casing and render the well unsuitable for monitoring. Well failures due to subsidence have been observed at Wilmington, California, Lake Maracaibo, Venezuela and other fluid withdrawal areas.

In view of the three monitoring problems cited above, we then looked at geothermal well completions to evaluate what types of geothermal wells may be susceptible to monitoring problems. Above the production zone, all U.S. geothermal wells are cased and cemented. In the Gulf coast areas, subsidence due to non-geothermal causes may cause casing slip or buckling in strata above the production zone. In other U.S. geothermal areas, casing performance above the production zone is not expected to be a problem. Within the producing zone, the completion method and associated potential measurement problems vary according to the type of geothermal resource. Vapor-dominated wells are unlined in the production zone, so casing compression is not a factor. Liquid-dominated wells generally use a slotted liner, perhaps with a gravel-pack. In these wells movements are expected to be small because of the anticipated use of brine reinjection for this type of field. Slip between casing and the ground may be a problem, particularly where no gravel packing is used. In geopressured wells, the production zone is cased, cemented and perforated. Large movements are expected which may threaten the installation due to casing collapse.

For the conditions where casing slip or collapse threatens the accuracy or stability of the monitoring installation, it is necessary to introduce flexibility into the casing. This may be done by the use of slip couplings or bellows sections. (See Section 3 for discussion of these techniques.)

\subsubsection{Available Extensometers and Inclinometers}

Displacement-measuring instruments used for geotechnical, geophysical, and subsidence monitoring applications were examined with respect to availability, performance characteristics, and installation and operating requi rements (Table 2.11). The instruments were then rated for their ability to make the measurement (axial or normal displacement) with the desired range and accuracy, and for their ability to cope with the unique temperature, corrosion, scaling and depth problems associated with geothermal environments (WCC,1977a). None of the available instruments were found suitable wi thout extensive modification for geothermal subsidence monitoring. The most promising of existing instruments are presented in Table 2.12 . 


\subsection{METHODS FOR IMPROVING EXISTING INSTRUMENTS}

\subsubsection{Corrosion Control}

Corrosion may reduce the service life of monitoring equipment and impair its operation. Permanently instal led instruments will experience the most severe conditions. A combination of corrosion-resistant techniques will be required. The best approach is to select an inherently corrosion resistant material. For long-term application in a geothermal environment, super-alloys such as Hastelloy-C and Inconel 625 will probably be the best choice. Austenitic stainless steels such as AL-6X and Type 316 will be adequa te for short immersion times. Cathodic protection by current impression may be a useful tool to protect long-term installations if current requirements are reasonable. The performance of most metals can be enhanced by the use of coatings and paints. However, the hazaras associated with coating/paint failure, particularly in permanent installations, seriously mitigates their usefulness in the harsh geothemal environment. Addition of corrosion/inhibitors to the geothermal fluid will probably reduce corrosion rates. The risk is that scaling or clogging of the producing formation may result (WCC, 1978a).

\subsubsection{Scaling Control}

In geothermal well instrumentation, problems may arise due to the build-up of silica $\left(\mathrm{SiO}_{2}\right)$ or calcite $\left(\mathrm{CaCO}_{3}\right)$ scale. The problems for instrumentation may include blockage or narrowing of the well, sealing of moving parts to the casing and blockage of well casing grooves. The control of geothermal scale has not been widely reported, and to date has largely been confined to removal and prevention after scaling was observed at damaging levels. Maintenance of a backpressure sufficient to prevent flashing appears to be the most successful approach. Since data on scale control are limited, the best approach is to design equipment to operate under conditions of moderate scaling and to accommodate periodic cleaning in the upper portions of the well (WCC, 1978a).

\subsubsection{Temperature Control - Insulation and Cooling}

Most instrumentation does not operate effectively at geothermal temperatures. One solution is to provide insulation and cooling for sensitive components. Vacuum-type insulation appears to be the most effective for geothemal conditions (Table 2.13). Dewar flasks are not as effective as multilayer foil insulation. However, multilayer insulation may pose some problems in the design of the supports to prevent crushing of the insulation. For a $1 \mathrm{~m}$ long, $100 \mathrm{~mm} \mathrm{0.D.} \mathrm{instrument} \mathrm{chamber,} \mathrm{maintained} \mathrm{at}$ $100^{\circ} \mathrm{C}$, heat flow was calculated to be 10 watts al lowing for heat leaks through support and electrical connections (WCC, 1978a).

For removal of this heat influx over a 10 hour to 100 hour logging cycle, several cooling systems were evaluated (WCC, 1978a). Two types of heat pumps, a mechanical heat pump that uses water as a refrigerant (Figure 2.1) and a themoelectric heat pump that uses nigh temperature thermoelectric materials ( $\mathrm{Pb}-\mathrm{Te}, \mathrm{Ge}-\mathrm{Te}, \mathrm{Ag}-\mathrm{Sb}$, or $\mathrm{Sn}-\mathrm{Te}$; Figure 2.2 ), may be able to 
provide the necessary cooling. Both reject heat to the well at temperatures in excess of ambient $\left(300^{\circ} \mathrm{C}\right)$. While these cooling devices have some drawbacks (scaling of moving parts for the mechanical heat pump, high current for the thermoelectric heat pump), maintenance of a constant temperature of $100^{\circ} \mathrm{C}$ would minimize many temperature-induced problems in electronic components.

\subsubsection{Temperature Control - Material Selection}

The most serious temperature effects on measurement of subsidence are probably length change, modulus change and electrical resistance change with temperature (WCC, 1978a). (Performance of electrical components is discussed in Section 2.3). In the design of geothermal subsidence monitoring equipment for use at $300^{\circ} \mathrm{C}$, the engineer must assess and attempt to minimize the potential effects of temperature on the response of the materials in the instrument. Not only must the probe body be temperaturecompensated to reduce length changes, but al so its component instrumentation, such as strain gauges, cantilevers, and pick-up coils, must be compensated in various ways to reduce errors due to changes in length, elastic modulus, and electrical resistance.

Many of the problems with length changes can be lessened by using controlled-expansion alloys such as $42 \% \mathrm{Ni}-\mathrm{Fe}$ (Table 2.14), by constructing the instrument using materials with equal expansion coefficients, and by using unstrained sensors near strained sensors to monitor temperature effects on signal levels. Note that Invar is not a low expansion alloy at geothermal temperatures (Figure 2.3). Similariy, a relatively new alloy, Super-Invar, looks promising for temperatures below $200^{\circ} \mathrm{C}$, but its thermal coefficient of expansion increases rapidly and non-linearly above that temperature (see Section 6.1 in Appendix A).

Elastic modulus changes can be reduced oy using controlled-modulus al loys such as Elvinar, Iso-elastic and $\mathrm{Ni}$-Span-C (Table 2.15a).

Electrical resistance changes can be reduced by using alloys with nearzero coefficients of resistance change (Table 2.15b). An unstrained gauge near a strained gauge can be used to compensate for short term (reversible) and long term (due to annealing) changes in resistance. Heat treatment helps reduce long term resistance changes.

\subsubsection{Other Materials and Operation Problems}

Other materials and operational problems associated with geothermal instrumentation were briefly assessed. Mechanical seal design is a major problem (Table 2.16; WCC, 1978a). Metal-to-metal seals are the best for geothermal use, but sometimes cannot be used because of electrical requirements. A second problem is limitations of electrical cables (WCC, 1978a). Nevertheless, the manufacture of an armored, insulated cable for geothermal environments appears to be within current technology. However, such cable will have to be custom-made. It will be costly and may require frequent repairs or replacement due to the harsh environment. Friction, tool bounce and cable stretch, and inclinometer orientation are depth- 
related problems (WCC, 1978a). No easy solution exists for the problem of friction of permanentiy installed extensometer rod/wire/pipe against the side of the borehole. Very straight holes may minimize frictional contacts, but may be hard to achieve in geothermal areas. The use of a three (or more) sensor probe which detects downhole markers significantly reduces the depth measuring problems associated with tool bounce and cable stretch (Figure 2.4). The use of gyroscopes appears to be the most promising solution for inclinometer orientation to replace the grooved casing and tracking wheels that orient conventional geotechnical inclinometers used for horizontal movement measurements.

\subsubsection{Recommended Materials and Techniques}

A summary of materials and techniques that can be used to improve existing equipment and design new ones is presented in Table 2.17. The most promising existing instruments, identified earlier, were re-rated, assuming that the recommended modifications were incorporated into the instrument. The instruments that received high ratings in this evaluation are presented in Table 2.18 .

\subsection{SENSOR AND SIGNAL TECHNOLOGY}

A number of instrumentation components were examined for their applicability to geothermal subsidence monitoring.

\subsubsection{Transducers}

A few transducers may be capable of providing accurate data at geothermal temperatures and may survive rough field conditions (Table 2.19; WCC, 1978b). Reed switch sensors offer the most promise because they require a minimum of downhole electronics and have a digital, "on-off" signal (Figure 2.5). Three gauges offer partial capabilities for geothermal measurement but require some high temperature signal conditioning:

(1) electrical resistance strain gauges and (2) LVDTs are available in high temperature packages but need high temperature signal conditioning to get a good signal to the surface, and (3) vibrating wire strain gauges provide a frequency signal only minimally subject to deterioration over long leads but need temperature compensation and sophisticated surface signal conditioning to excite and pick-up the wire vibration frequency.

\subsubsection{Signal Conditioning Equipment}

Figure 2.6 shows some possible signal conditioning systems (see also WCC, 1978b). High temperature components are available to construct signal conditioners for downhole operation (Table 2.20 and Section 6.2 in Appendix A). Analog-to-digital (A-D) conversion is desirable to minimize signal degradation over long lead wires for analog-type signals. However, the accuracy of the $A-D$ conversion must be evaluated, even with high temperature components, over the the $20^{\circ} \mathrm{C}$ to $300^{\circ} \mathrm{C}$ temperature range. 


\subsubsection{Data Transmission Equipment}

Hard wire telemetry using multiplexing techniques can reduce cable requirements (WCC, 1978b). Accuracy of the transmitted data will be dependent on the sampling rate and the high temperature performance of the multiplexing equipment. The benefits of reduced cable replacement costs and improved performance of mono-conductor cable (see Appendix A-6.2.3) will have to be balanced against the problems of the more complex electronics associated with telemetry use.

\subsubsection{Data Processing Equipment}

A variety of data processing systems were reviewed (Table 2.21; WCC, 1978b). Simple signal conditioning equipment interfaced to a strip chart recorder or event recorder is probably adequate for initial instrument testing. Magnetic tape recording or more sophisticated data acquisition systems (Figure 2.7 ) may merit consideration after the instrument is debugged.

\subsection{ADAPTION OF PRODUCING WELLS TO INSTRUMENTATION NEEDS}

It is probably economical to use producing wells for monitoring since no (or minimal) additional expense is incurrea for drilling. Also, if just a few monitoring-only wells are installed, the zone of maximum compaction may be missed if unusual subsidence patterns develop, as at Wairakei. The adaption of production wells to monitoring will permit a broader area of the geothermal field to be studied.

In some geothermal wells (in particular vapor-dominated wells), the producing zone is unlined. The use of radioactive bullet markers combined with a suitable gamma-ray detector probe was determined to be a potentially workable system for unlined wells. Radioactive bullets may also find use in cased wells where movements of the slotted liner or casing are not expected to be representative of formation movements. (Refer to Section 2.1.2.) Cased or lined wells with good casing/formation adhesion offer many options for monitoring. However, there is a need to introduce flexibility into conventional well casing to key it to the adjacent ground and prevent casing failures in zones of large movements. The use of slip couplings (Figure $2.8 \mathrm{a}$ and $\mathrm{b}$ ) and the use of Dellows sections (Figure 2.9) along the length of the casing were considered in Section 2.1.2. Bellows sections may be installed at regular intervals using an oil industry hydraulic corrugating tool (modified casing expander) once the casing is in place. However, bellows sections may enhance corrosion and promote collapse due to stress concentrations. Slip couplings, although used in the oil industry, are not yet available for geothermal use (see Appendix A-4.0).

To minimize interference with fluid production, two techniques were proposed. An access gland to allow entry of a settlement probe while maintaining flow, pressure and temperature in a producing well is shown on Figure 2.10. Access glands are used in the oil industry, and special designs have been developed for geothermal logging (Veneruso, 1979). Even 
with an access gland, it may still be necessary to shut in the well if the flowing fluid causes the probe to bounce as it is lowered. This approach is used in the oil industry. The access gland serves to maintain well temperature and pressure in that case.

If it proves undesirable to use the production casing for monitoring; small (25-50 mm) diameter auxiliary casing is proposed. This casing, strapped outside the production casing and installed with it, has no flow and allows monitoring without interference with geothemal filuid production (see Figure 2.11). However, its use may complicate driliting and cementing operations and may be very costly.

\subsection{NEW CONCEPTS IN SUBSIDENCE INSTRUMENTATION}

A number of new instrumentation concepts were proposed. Although many of the new concepts draw on techniques and materials discussed in earlier reports, each of the monitoring methods and well modifications presented includes new features to improve the ease and/or accuracy of subsidence measurement.

\subsubsection{New Concept for Monitoring in Production Well Casing}

A multi-sensor reed switch settlement probe is proposed for use in cased production wells (Figure 2.12). The casing would incorporate magnet markers at 15-20 m intervals and slip couplings (or bellows sections) for flexibility. The instrument system provides direct readout to the nearest $25 \mathrm{~mm}$ by using a series of closely spaced reed switches at each end of the probe. With the addition of a precision electronic timer, distances can be measured to $0.1 \mathrm{~mm}$, although irregular probe speed may not allow the full accuracy to be realized in the field.

\subsubsection{New Concepts for Monitoring in Auxiliary Casing}

Five monitoring methods using probes and one fluid settlement device were developed in concept for use in auxiliary casing. Key features of these instruments are as follows:

- Single Sensor Reed Switch Probes - - This probe uses casing with strain-free liner segments so in-situ calioration is obtained without the use of a 3 -sensor probe (Figure 2.13).

- Triple Sensor Oscillator-Type Magnet Detector -- More sensitive than a reed switch, it may have a better chance of detecting magnetic markers at $300^{\circ} \mathrm{C}$, at which sensitivities of all magnet detectors decrease. (See Section 3, Figure 3.3).

- Single Sensor Vibrating Wire Probe -- Permanently installed vibrating wires are monitored by a portable probe. This system monitors strain without depth or distance measurement, so results are unaffected by tool bounce or cable stretch (Figure 2.14). 
- Single Sensor Proximity Detector Probe -- The probe counts closely spaced corrugations on auxiliary casing between adjacent couplings. In-situ calibration is provided by strain-free liner segments. The system gives direct readout of subsidence to the nearest $12 \mathrm{~mm}$ (Figure 2.5).

- Triple Sensor Weigand Device Probe -- Another type of magnet detector, it is available in commercial modules for use to $250^{\circ} \mathrm{C}$ (Figure 2.16).

- Double Fluid Settlement Device -- The surface-monitored pressure of a slug of mercury as it moves through the system displacing nitrogen is proportional to the distance between adjacent reference points. No downhole probes, wires, or rods are needed (Figure 2.17).

\subsubsection{Comparative Rating of New Concepts}

The seven new instrument methods were comparatively rated. Several of the instruments were suitable for use in a variety of the proposed casings, but were discussed in reference to one casing type only. To take into account the effect of casing, each instrument was rated for several casing types, where appropriate. The most promising new instrument concepts meriting further consideration for development were identified as:

- Multi-Sensor Reed Switch Probe (for use in modified production casing).

- Multi-Sensor Reed Switch Probe (for use in simple auxiliary casing).

- 3 Sensor Oscillator-Type Magnet Detector Probe (for use in modified production casing). 


\section{TABLE 2.1}

\section{COMPARISON OF GEOTHERMAL SUBSIDENCE AREAS}

(From Atherton and Others, 1976)

$\begin{array}{cl}\text { Wafrakei } & \text { Broadlands } \\ \text { New Zealand } & \text { New Zealand }\end{array}$

Kawerau New Zealand

Hew Zea New Zealand

SURFACE DEFORMATION

Subsidence

Area Affected
Maximum Subsidence
Subsidence Rate

$65 \mathrm{~km}^{2}(1956-1970)$

4.7m (1956-1974)

Max. 40-45 cm/yr (1967-1974)

Horizontal Movement

Maximum Movement
Rate

\section{Associated Faulting}

Time of Principal Occurrence

RESERVOIR MATERIALS

Type, Age, Consolidation

Porosity

Permeability

Compaction Behavior

OVERBURDEN

Type, Competence, Deformation Properties

REGIONAL GEOLOGIC STRUCTURE

Folding, Faulting, Regional Stress

PRODUCTION

Depth
Fluld Type
Temperature
Production Rate
Total Fluid Withdrawal
Maximum Pressure Decline
Subsidence: Head Decline

Fiuld Type

\begin{abstract}
$0.8 m(1956-1974)$
$\operatorname{Max} .-10 \mathrm{~cm} / \mathrm{yr}(1970)$

None

1953-1976 +

Pumice breccia with sands tone and minor silts tone, medial rhyolite sill, Pleis tocene

Extremely variable due to cementation

< 1-30 mD (Intergranular) $\geq 10$ (fracture)

Maxinum subsidence outside area of greatest production. Local sills of volcanic rock may prevent transfer of load to underlying reservoir
\end{abstract}

Tuffaceous shale and sandstone with interbedded tuff and conglomerate, Pleis tocene

Sediments drape-folded over basement highs, normal

faulting with small component of right-lateral movement, Indicates tensile stress

\section{0-1360m}

Hot whter and flashed

steam

Maximum $260^{\circ} \mathrm{C}$

$1.3 \times 10^{7} \mathrm{~kg} /$ day (1975)

$930 \times 10^{9} \mathrm{~kg}(1956-1974)$

$25 \mathrm{~kg} / \mathrm{cm}^{2}(1956-1974)$

$.02(1956-1974)$
$73 \mathrm{~km}^{2}(1969-1975)$

$0.175 \mathrm{~m}(1969-1975)$

$7.5 \mathrm{~cm} / \mathrm{yr}(1969-1972)$

$0.12 m(1969-1975)$

Avg. $2 \mathrm{~cm} / \mathrm{yr}$ (1969-1975)

None

$1966-1976+$

Pumice breccia and tuff, Pleistocene, with medial siltstone and rhyolite aquitards, Pleis tocene

15-50\% (highest in punice breccta)

Moderately high

Chiefly siltstone, mudstone, and rhyolite with minor sands tone, tuff and alluvium. Pieis tocene

Normal faulting, with small component of right-lateral movement. indicates tensile stress

$430-1200 \mathrm{~m}$

Hot water and flashed steam

Maximum $3000^{\circ} \mathrm{Ct}$

Avg. $2.63 \times 10^{7} \mathrm{~kg} / \mathrm{day}$ (1973-1975)
$-1 \mathrm{~km}^{2}$

$0.028(1970-1971)$

Max. $2.8 \mathrm{~cm} / \mathrm{yr}$ (1970-1972)

Not measured

None

$1956-1976+$

Pumice breccia and ignimorite, with medial andesite sills. Pleistocene

Pleistocene rhyolite flows and Holocene (?) alluvium

Numerous normal faults, with smal component of right-lateral movement, Indicating tenstle stress

460-915m

Hot water and flashed steam

Maximum $270^{\circ} \mathrm{C}+$

$\operatorname{Max} .1 .6 \times 10^{7} \mathrm{~kg} /$ day $(1956-1970)$

+ Sources: Grindley, 1964, 1970; Stilwell and others. 1975. 
TABLE 2.2

COMPARISON OF GROUNDWATER SUBSIDENCE AREAS

(From Atherton et al, 1976)

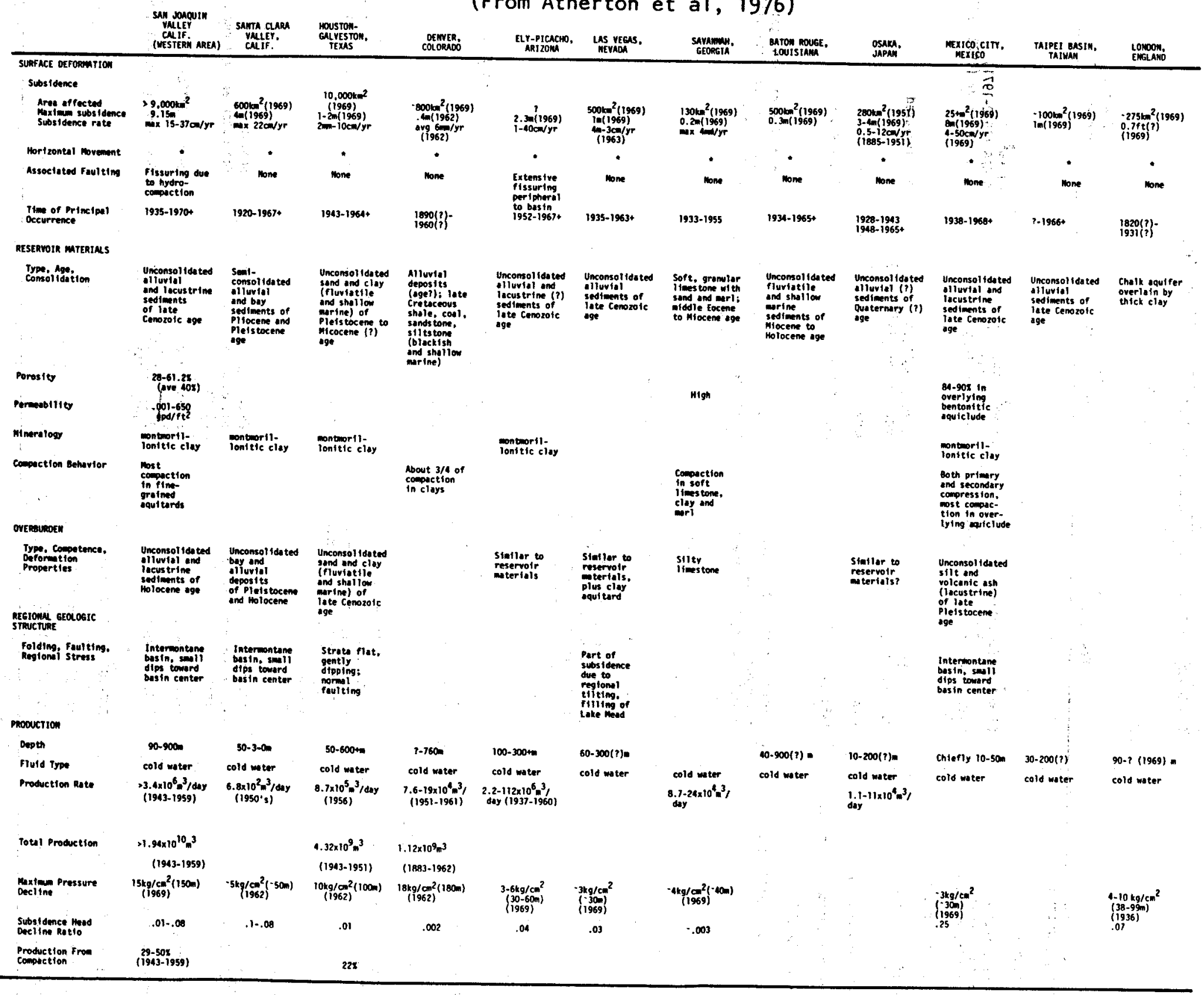


TABLE 2.3

COMPARISON OF MAJOR OIL AND GAS SUBSIDENCE AREAS

(From Atherton et al, 1976)

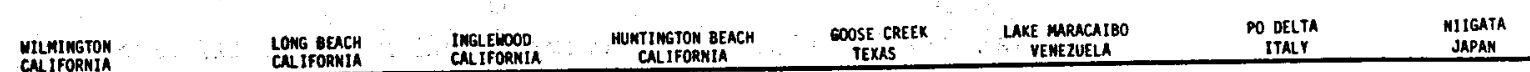

SURFACE DEFORMATION:

Subsidence

$\begin{array}{lc}\text { Subsidence } & \\ \text { Area Affected } & >65 \mathrm{~km}^{2}(1970) \\ \text { Maximim Subsidence } & 8.8 \mathrm{man}(1928-1970) \\ \text { Subsidence Rate } & 33-77 \mathrm{~cm} / \mathrm{yr} \\ (1947-1958 ;\end{array}$

Subsidence Rate $\quad \begin{gathered}33-77 \mathrm{~cm} / \mathrm{yr} \\ (1947-1958 ;\end{gathered}$

before injection)

Horizontal Movement

Maximum Rovenent

Rate

3. $\operatorname{mon}(1937-1970)$

culifornia

TEXRS

VENEZUELA

Associsted Faulting

$\begin{array}{lc}\text { Type } & \text { Low angle } \\ \text { Location } & \text { Central } \\ \text { Earthquake } & 6(1947-1961) \\ \text { Magnl tude } & 2.4-3.3\end{array}$

Time of Principat Occurrence

REsERvo:R MTERIaLs

Type, Ape,
Consolidation

1947-1958+ (now

$31 \mathrm{ka}^{2} \quad 11 \mathrm{~km}^{2} \quad 37 \mathrm{~km}^{2}$

$0.75 m(1925-1967)$

$1.73 m(1911-1963) \quad 1.22 m(1933-1965)$

vvg. $1.8 \mathrm{~cm} / \mathrm{yr} \quad$ avg. $3.3 \mathrm{~cm} / \mathrm{yr} \quad$ ovg $3.8 \mathrm{~cm} / \mathrm{yr}$

$10 \mathrm{~km}^{2}$

in(1918-1925)

400-700 $\mathrm{km}^{2}$

$.400 \mathrm{~km}^{2}$

$0.3 \cdot 3.3 n(1926-1954)$

$2 \pi$

$0.8 m(1900-1960)$

avg $14 \mathrm{~cm} / \mathrm{yr}$

$30 \mathrm{~cm} / \mathrm{yr}(1930 \cdot \mathrm{s})$

$1-3 \mathrm{~cm} / \mathrm{yr}(1890-1950)$
$\max 30 \mathrm{~cm} / \mathrm{yr}$

$40-50 \mathrm{~cm} / \mathrm{yr}$

$0.76 m(1934-1963)$

Unconsolldated to
semitconsolfdeted.
sand with inter.

added clay and

shale (marine).
Hiocene to

Pllocene Age

Porosity 20-35x

Parmesbiliter $\quad 100+1500$ mo

compaction Beharfor $60 x$ of compaction

fn sands, $40 x$ th
clays ond shales

OVERBUROEN

Type, Competence
Defornation Properties

Unconsolldated

shale, sand,

claystone. of

plipcene and
pleis tocene age

avg. $2.6 \mathrm{~cm} / \mathrm{yr}$

Wone N1gh angle, nomal Nore

Portpheral

Wigh angle, normal
Peripheral

None

None

(.

1925-1967 1911-1963 1933-1965

$1917-1926$

$1950-1960$

$1955-1960+$

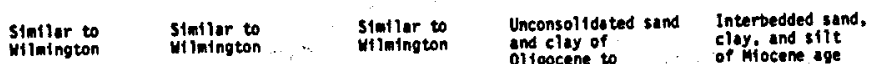

ollgocene to

Unconsolldated

celcareous sands

with interbedded

and lagoonal)

Deconsoll lated

sand, sands tone

with interbedded
clay, Cenozoic age Quaternary age

Compaction

roughly equal

in sands
clays

Cause of some

to development

is unknown

\begin{tabular}{|c|c|}
\hline $\begin{array}{l}\text { Simfler to } \\
\text { wilutington }\end{array}$ & $\begin{array}{l}\text { Similar to } \\
\text { Wilmington }\end{array}$ \\
\hline
\end{tabular}

Similar to
reservoir rock?

Similer to
reservoir rock?

Wiluington Wilmington wiluington reservolr rock

REGLONAL GEOLOGIC STRUCTURE

Folding, Foulting.

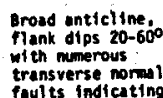

Tightly folded Anticline;

Anticline
fouiting?

Gentle dome in

younger and

younger sediments:
older deposits

older deposits
intensely faulted.

faults indicating fulting

conpressive stress

PRODUCTION

Depth

Fiuld type

$600-2300 m$

(most $600-1100 \mathrm{~m})$

ot1., water. and

wedtan $1690 \mathrm{~m}$

median $900 \mathrm{~m}$

median $930 m$

o11 und water oll; water and gas?

200-1400m

ot1. gas, and otl and water (?)

I(one well 700m)

$1-1000 \mathrm{~m}$

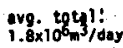

brine with dissolved brine with
methane

Production Rate

o11: $11-22 \times 10^{3} m^{3} / d a y$

Total Production

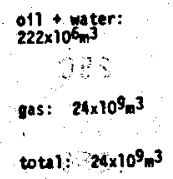

ofi(plus water?

1917-1926):

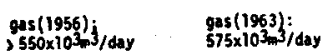

Totu)(1956), if Total (1956), if

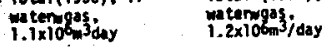

Moxtmum iressure Deciline in $77 \mathrm{~kg} / \mathrm{cm}^{2}(1100051)$

Substdence: Head Decitine

0.01

Production from compaction

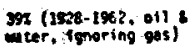

Notes:

1) Dates in parentheses indicate period of motsurement or year of noport

2) Densities used for conversions, at $250^{\circ} \mathrm{C}$ and 1 atminatural gas, $0.829 \mathrm{~kg} / \mathrm{m}^{3}$ : $011.858 \mathrm{~kg} / \mathrm{m}^{3}$; weter. $1000 \mathrm{~kg} / \mathrm{m}^{3}$

2. Not neasured

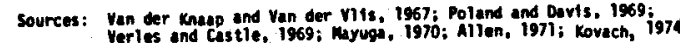


TABLE 2.4

REPRESENTATIVE GEOTHERMAL RESERVOIR TEMPERATURES

(Modified from Ellis, 1975; average and maximum temperatures in major fields around the world)

\begin{tabular}{|c|c|c|}
\hline SYSTEM & $\begin{array}{c}\text { Average } \\
\text { Temperature } \\
\left({ }^{C}\right)\end{array}$ & $\begin{array}{c}\text { Maximum } \\
\text { Tempgrature } \\
(\langle C)\end{array}$ \\
\hline $\begin{array}{l}\text { HYDROTHERMAL } \\
\text { CONVECTION SYSTEMS }\end{array}$ & & \\
\hline \multicolumn{3}{|l|}{ VAPOR-DOMINATED } \\
\hline $\begin{array}{l}\text { USA } \\
\text { The Geysers }\end{array}$ & 230 & 285 \\
\hline $\begin{array}{l}\text { ITALY } \\
\text { Larderello }\end{array}$ & 200 & 260 \\
\hline Mt. Amiata & 170 & 190 \\
\hline $\begin{array}{l}\text { JAPAN } \\
\text { Matsukawa. }\end{array}$ & 220 & 270 \\
\hline \multicolumn{3}{|l|}{ LIQUID-DOMINATED } \\
\hline $\begin{array}{l}\text { NEW ZEALAND } \\
\text { Wairakei }\end{array}$ & 230 & 260 \\
\hline Kawerau & 250 & 285 \\
\hline Broadlands & 255 & 300 \\
\hline $\begin{array}{l}\text { ICELAND } \\
\text { Namafjall }\end{array}$ & 250 & \\
\hline $\begin{array}{l}\text { USSR } \\
\text { Pauzhetsk }\end{array}$ & 185 & \\
\hline $\begin{array}{l}\text { USA } \\
\text { Niland } \\
\text { (Salton Sea) }\end{array}$ & $300+$ & \\
\hline $\begin{array}{r}\text { EL SALVADOR } \\
\text { Ahuachapan }\end{array}$ & 230 & 250 \\
\hline $\begin{array}{l}\text { JAPAN } \\
\text { Otake }\end{array}$ & 230 & 250 \\
\hline N. Hachimantai & --- & $>250$ \\
\hline Hatchobaru & 250 & 300 \\
\hline Ontkobe & $\cdots$ & 288 \\
\hline $\begin{array}{l}\text { PHILLIPINES } \\
\text { S. LUZON }\end{array}$ & $\cdots$ & \\
\hline $\begin{array}{l}\text { TURKEY } \\
\text { Kilzidere }\end{array}$ & 190 & 220 \\
\hline $\begin{array}{l}\text { CHILE } \\
\text { EI Tatio }\end{array}$ & 230 & 260 \\
\hline
\end{tabular}


TABLE 2.5

PRODUCTION-INDUCED CHANGES IN THE

AVERAGE TEMPERATURE OF GEOTHERMAL RESERVOIRS

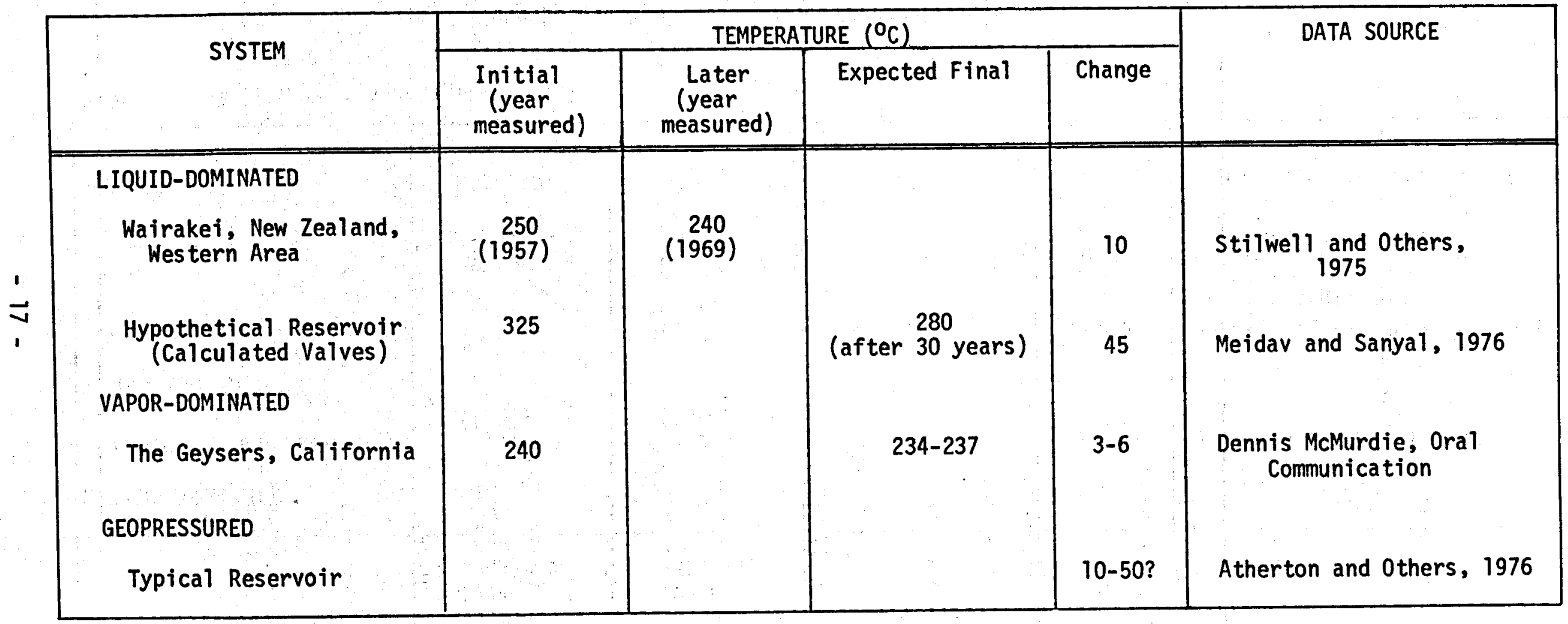

Modified after Atherton and Others (1976) 
TABLE 2.6 GEOTHERMAL RESERVOIR PRESSURE CHANGES

(a) PRODUCTION-INDUCED CHANGES IN AVERAGE GEOTHERMAL RESERVOIR PRESSURE

\begin{tabular}{|c|c|c|c|c|c|}
\hline SYSTEM & \multicolumn{4}{|c|}{ PRESSURE $\left(\mathrm{kg} / \mathrm{cm}^{2}\right)$} & \multirow[t]{2}{*}{ DATA SOURCE } \\
\hline 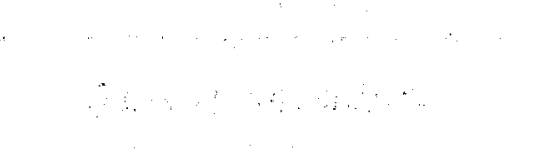 & $\begin{array}{l}\text { Initial } \\
\text { (year } \\
\text { measured) }\end{array}$ & $\begin{array}{l}\text { Later } \\
\text { (year } \\
\text { measured) }\end{array}$ & Expected Final & Change & \\
\hline \multicolumn{6}{|l|}{ LIQUID-DOMINATED } \\
\hline Wairakei, New Zealand & $\begin{array}{c}\sim 52 \\
(1957)\end{array}$ & $\begin{array}{c}\sim 27 \\
(1974)\end{array}$ & & $\sim 25$ & Stilwell and Others, 1975 \\
\hline VAPOR-DOMINATED & & & & & \\
\hline The Geysers, California & & $\begin{array}{l}32-35 \\
(1969)\end{array}$ & $\sim 6$ & $26-29$ & $\begin{array}{l}\text { Koenig, 1969; McMurdie, oral } \\
\text { communication }\end{array}$ \\
\hline$\because, \cdots$, & $\begin{array}{l}13.6 \\
(1957)\end{array}$ & $\begin{array}{l}10-0 \\
(1967)\end{array}$ & & $3-6$ & Ramey, 1968 \\
\hline GEOPRESSURED & $390-540$ & & 140 & $250-400$ & Papadopulos and Others, 1975 \\
\hline
\end{tabular}

(Modified after Atherton and Others, 1976)

(b) PROBABLE RANGE OF MAXIMUM PRESSURE CHANGES

\begin{tabular}{|lc|}
\hline $\begin{array}{l}\text { Geothermal Field Type } \\
\text { vapor-dominated systems }\end{array}$ & $\begin{array}{l}\text { Pressure Change } \\
\text { liquid-dominated systems }\end{array}$ \\
geopressured systems & $50-250 \mathrm{Kg} / \mathrm{m}^{2}$ \\
\hline
\end{tabular}

Note: Based on current generating requirements 
TABLE 2.7

CHEMICAL COMPOSITION OF GEOTHERMAL WATERS, in $\mathrm{mg} / 1$

(From Various Localities in the Western United States and Mexico; after Chen, 1975)

\begin{tabular}{|c|c|c|c|c|c|c|c|c|c|c|c|c|c|c|c|c|c|c|c|c|}
\hline Place & $\mathrm{pH}$ & $\begin{array}{c}\text { TDS mea- } \\
\text { sured }\end{array}$ & $\begin{array}{l}\text { Sus: } \\
\text { Solid }\end{array}$ & $\mathrm{Na}$ & K & $\mathrm{Ca}$ & $\mathrm{Mg}$ & $L i$ & $\mathrm{Cl}$ & $\mathrm{HCO}_{3}$ & $\mathrm{CO}_{3}$ & $\mathrm{SO}_{4}^{-}$ & B & $\mathbf{F}$ & $\begin{array}{l}\text { Sui- } \\
\text { flde }\end{array}$ & $\mathrm{NO}_{3}$ & $\mathrm{NH}_{4}(\mathrm{~N})$ & si & $\begin{array}{c}\text { Total } \\
\mathrm{Si}\end{array}$ & $\begin{array}{l}P 0 \\
(P)^{4}\end{array}$ \\
\hline $\begin{array}{l}\text { Brady Hot } \\
\text { Springs (Mev.) }\end{array}$ & 8.6 & 3130 & 6.0 & 1070 & 51 & 19.5 & 0.075 & 3.0 & 1610 & 10.0 & 0.15 & 242 & 8.2 & 3.1 & NA & 0.47 & 0.3 & 84 & 85 & 0.01 \\
\hline $\begin{array}{l}\text { Surprise } \\
\text { Valley (CA) }\end{array}$ & 8.8 & 1310 & 80 & 437 & 30 & 0.05 & 0.01 & 0.5 & 234 & 111 & 3.0 & 351 & 6.0 & 7.4 & $N A$ & 2.0 & 0.10 & 97 & 130 & 0.02 \\
\hline $\begin{array}{l}\text { Beowove } \\
\text { Hell } \# \text { (Nev.) }\end{array}$ & 9.66 & 1170 & 10.1 & 240 & 35 & 0.1 & 0.01 & 1.9 & 66 & 89.3 & 16.2 & 138 & 2.25 & 16 & NA & 16.8 & 0.3 & 230 & 234 & 0.02 \\
\hline $\begin{array}{l}\text { Beowowe } \\
\text { Hell } \# 2 \text { (Nev.) }\end{array}$ & 9.53 & 1620 & 2.8 & 250 & 35 & 0.1 & 0.01 & 1.9 & 65.5 & 117 & 15.8 & 210 & 2.22 & 17.8 & NA & 2.44 & 0.2 & 230 & 234 & 0.02 \\
\hline $\begin{array}{l}\text { Mabuska } \\
\text { Wel1 } \| \text { (Nev.) }\end{array}$ & 8.76 & 1050 & 0.5 & 310 & 16.5 & 12.8 & 0.1 & 0.3 & 50 & 37.5 & 0.80 & 610 & 0.96 & 7.25 & 0.36 & -- & 0.10 & 56 & 59 & ---- \\
\hline $\begin{array}{l}\text { Wabuska } \\
\text { Well } \# 2 \text { (Nev.) }\end{array}$ & 8.3 & 1100 & 0.5 & 340 & 16.5 & 12.8 & 0.1 & 0.3 & 47.5 & 49.0 & 0.39 & 590 & 1.08 & 7.30 & 0.33 & 0.33 & 0.10 & 53 & 53 & $\cdots$ \\
\hline $\begin{array}{l}\text { Steamboat } \\
\text { Springs (Nev.) }\end{array}$ & 8.5 & 1980 & 1.5 & 615 & 62.5 & 0.3 & 0.36 & 8.2 & 730 & 232 & 2.9 & 103 & 43.5 & 1.7 & 4.42 & 0.4 & 0.10 & 118 & 121 & $\cdots$ \\
\hline $\begin{array}{l}\text { Geysers } \\
5 \& 6(C A)\end{array}$ & 7.5 & 1210 & 1.1 & 0.25 & 0.05 & 0.35 & 0.03 & $\cdots$ & 13.5 & 199 & $\cdots$ & 138 & 456 & 0.2 & 0.9 & 0.83 & 122 & 0.4 & 0.5 & 0.003 \\
\hline $\begin{array}{l}\text { Geysers } \\
8 \quad(C A)\end{array}$ & 7.6 & 575 & 0.8 & 0.25 & 0.05 & 138 & 0.065 & --- & 1.0 & 116 & --- & 122 & 232 & 0.22 & 1.5 & 1.2 & 66.2 & 0.32 & 0.32 & $\cdots$ \\
\hline $\begin{array}{l}\text { Geysers } \\
9 \& 10 \text { (CA) }\end{array}$ & $=7.0$ & 164 & 0.7 & 0.25 & 0.05 & 1.85 & 0.03 & -.-- & 1.0 & 16.2 & $\cdots$ & 33 & 52 & 0.38 & 0.46 & 5.6 & 14.5 & 0.56 & 0.56 & ---- \\
\hline $\begin{array}{l}\text { Geysers } \\
11^{(C A)}\end{array}$ & 7.6 & 400 & 0.6 & 0.5 & 0.05 & 1.2 & 0.03 & --- & 1.0 & 176 & $-\cdots$ & 78 & 106 & 0.12 & 0.68 & 0.72 & 77.5 & 0.47 & 0.47 & $\cdots$ \\
\hline $\begin{array}{l}\text { Cerro Prieto } \\
\text { Mexico }(x)\end{array}$ & NA. & 22600 & 6.0 & 6700 & 890 & 600 & 13 & 13.0 & 11700 & NA & NA & 6.4 & 17 & 0.5 & NA & 34.4 & 15.6 & 156 & 250 & 0.04 \\
\hline $\begin{array}{l}\text { Cerro Prieto } \\
\text { Mexico }(Y)\end{array}$ & 7.0 & 77400 & 90 & 25600 & 4660 & 2000 & 234 . & 21.0 & 44400 & 97 & $\cdots$ & 260 & 50 & 3.4 & NA & 1.6 & 0.1 & 60 & 60 & 0.04 \\
\hline $\begin{array}{l}\text { Cerro Prieto } \\
\text { Mexico (W) }\end{array}$ & 5.2 & 660 & 2.3 & 5.0 & 1.0 & 3.1 & 0.028 & 0.01 & 8.9 & 20.1 & --- & 470 & 0.93 & --- & NA & 45.1 & 147 & 0.95 & 1.0 & --- \\
\hline
\end{tabular}


TABLE 2.8

SPECIFICATIONS FOR MONITORING SUBSURFACE VERTICAL DISPLACEMENTS

\begin{tabular}{|c|c|c|c|}
\hline $\begin{array}{l}\text { TYPE OF GEOTHERMAL } \\
\text { RESOURCE } \\
\begin{array}{l}\text { MEASUREMENT } \\
\text { CAPABILITY }\end{array}\end{array}$ & $\begin{array}{l}\text { VIPOR } \\
\text { DOMINATED }\end{array}$ & $\begin{array}{l}\text { LIQUID- } \\
\text { DOMINATED }\end{array}$ & $\begin{array}{l}\text { GEO- } \\
\text { PRESSURED }\end{array}$ \\
\hline $\begin{array}{l}\text { MAXIMUM DEPTH OF MEASURE- } \\
\text { MENT }\end{array}$ & $\begin{array}{cc}3 \mathrm{~km} \\
(6 \mathrm{~km})\end{array}$ & $\left(\begin{array}{ll}3 \mathrm{~km} \\
(6 \mathrm{~km})\end{array}\right.$ & $(6-7 \mathrm{~km})$ \\
\hline MAXIMUM TOTAL COMPACTION & $\begin{array}{l}0.3 \mathrm{~m} \\
(0.6 \mathrm{~m})\end{array}$ & $\left(\begin{array}{ll}4 & \mathrm{~m} \\
6 & \mathrm{~m}\end{array}\right)$ & $(5-7 \mathrm{~m})$ \\
\hline $\begin{array}{l}\text { DESIRED MAXIMUM VERTICAL } \\
\text { INTERVAL BETWEEN } \\
\text { MONITORING POINTS }\end{array}$ & $\begin{array}{l}30 \mathrm{~m} \text {, increas - } \\
\text { Ing to } 80 \mathrm{~m} \\
\text { outside reser- } \\
\text { voir }\end{array}$ & $\begin{array}{l}30 \mathrm{~m} \text {, increas- } \\
\text { ing to } 80 \mathrm{~m} \\
\text { outside reser- } \\
\text { voir }\end{array}$ & $\begin{array}{l}\text { (30 m, Increas - } \\
\text { Ing to } 80 \mathrm{~m} \\
\text { outs } 1 \text { de reser - } \\
\text { voir) }\end{array}$ \\
\hline $\begin{array}{l}\text { ACCURACY OF MEASUREMENT } \\
\text { OVER FULL DEPTH OF } \\
\text { INSTALLATION }\end{array}$ & $\pm 3 \mathrm{~mm}$ & $\pm 30 \mathrm{~mm}$ & $( \pm 30 \mathrm{~mm})$ \\
\hline $\begin{array}{l}\text { ACCURACY OF MEASUREMENT } \\
\text { OVER } 30 \mathrm{M} \text { INTERVAL } \\
\end{array}$ & $\pm 0.5 \mathrm{~mm}$ & $\pm 5 \mathrm{~mm}$ & $( \pm 5 \mathrm{~mm})$ \\
\hline $\begin{array}{l}\text { MINIMUM FREQUENCY } \\
\text { OF REAOINGS }\end{array}$ & $\begin{array}{l}\text { weekly for } \\
\text { lst month: } \\
\text { monthly for } \\
\text { lst years } \\
\text { then seml- } \\
\text { annualiy }\end{array}$ & $\begin{array}{l}\text { weekly for } \\
\text { lst month; } \\
\text { monthly for } \\
\text { lst year; } \\
\text { then semf- } \\
\text { annualiy }\end{array}$ & $\begin{array}{l}\text { (weekly for } \\
\text { lst month: } \\
\text { monthly for } \\
\text { ist year: } \\
\text { then semi- } \\
\text { annually) }\end{array}$ \\
\hline MONITORING PERIOD & $\begin{array}{c}15 \text { yrs } \\
(50 \text { yrs })\end{array}$ & $\begin{array}{l}15 \text { yrs } \\
(50 \text { yrs })\end{array}$ & (15 yrs) \\
\hline $\begin{array}{l}\text { TYPE OF GEOTHERMAL } \\
\text { ENVIRON- } \\
\text { MENTAL CAPABILITY }\end{array}$ & $\begin{array}{l}\text { VAPOR- } \\
\text { DOMINATED }\end{array}$ & $\begin{array}{l}\text { LIQUID- } \\
\text { DOMINATED }\end{array}$ & $\begin{array}{l}\text { GEO- } \\
\text { PRESSURED }\end{array}$ \\
\hline TEMPERATURE & $\left(285^{\circ} \mathrm{C}\right)$ & $\left(300^{\circ} \mathrm{C}\right)$ & $\left(375^{\circ} \mathrm{C}\right)$ \\
\hline PRESSURE & $35 \mathrm{~kg} / \mathrm{cm}^{2}$ & $\begin{array}{l}300 \mathrm{~kg} / \mathrm{cm}^{2} \\
\left(600 \mathrm{~kg} / \mathrm{cm}^{2}\right)\end{array}$ & $\begin{array}{c}(800-1,000 \\
\left.\mathrm{kg} / \mathrm{cm}^{2}\right)\end{array}$ \\
\hline SALINITY & $0.01 \%$ & $\begin{array}{l}3 \% \\
(20 \%)\end{array}$ & $\begin{array}{l}\text { (3x, increas. } \\
\text { ing to up to } \\
20 \% \text { above reser. } \\
\text { voir) }\end{array}$ \\
\hline DISSOLVED SOLIOS & $0.2 \%$ & $30 \%$ & (more than $30 \%)$ \\
\hline
\end{tabular}

Note: Long-term values in parentheses 
TABLE 2.9

SPECIFICATIONS FOR MONITORING

SUBSURFACE HORIZONTAL DISPLACEMENTS

\begin{tabular}{|c|c|}
\hline $\begin{array}{l}\text { MEASUREMENT } \\
\text { CAPABILITY }\end{array}$ & All Cases \\
\hline $\begin{array}{l}\text { MAXIMUM ANGULAR ROTATION } \\
\text { OVER DEPTH }\end{array}$ & $1^{0}$ \\
\hline $\begin{array}{l}\text { MAXIMUM LOCAL ANGULAR } \\
\text { ROTATION }\end{array}$ & $10 \mathrm{deg}$ \\
\hline $\begin{array}{l}\text { ACCURACY OF ANGULAR } \\
\text { ROTATION MEASUREMENT }\end{array}$ & $40 \mathrm{sec}$ \\
\hline 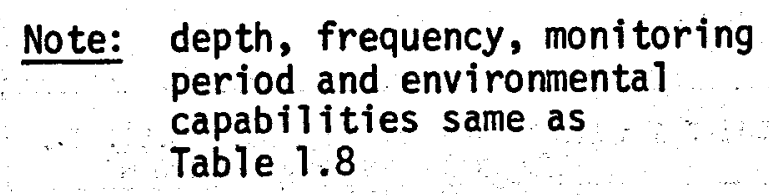 & \\
\hline
\end{tabular}


TABLE 2.10

RELATION BETWEEN SUBSIDENCE PARAMETERS AND WELL CASING PROTRUSION

(Selected cases from Poland \& Davis, 1969)

\begin{tabular}{|c|c|c|c|c|c|c|}
\hline LOCATION & GEOLOGY & $\begin{array}{c}\text { MAXIMUM } \\
\text { SUBSIDENCE }\end{array}$ & $\begin{array}{c}\text { TIME } \\
\text { DURATION } \\
\text { OF } \\
\text { SUBSIDENCE }\end{array}$ & $\begin{array}{l}\text { ANNUAL } \\
\text { RATE }\end{array}$ & $\begin{array}{c}\text { THICKNESS } \\
\text { OF } \\
\text { SUBSIDENCE } \\
\text { ZONE }\end{array}$ & $\begin{array}{c}\text { WELL } \\
\text { CASING } \\
\text { PROTRUSION }\end{array}$ \\
\hline Wilmigton, Calif. & $\begin{array}{l}\text { Sediments (Recent to } \\
\text { Miocene) } 6000 \mathrm{ft} \text { thick. } \\
\text { Over basement schist } \\
\text { (Pre-tertiary) } \\
\text { Oil Production zone } \\
\text { from 2500-6000 ft } \\
\text { depth. Shales, silt- } \\
\text { stone, sands \& sand- } \\
\text { stone. }\end{array}$ & $8.1 \mathrm{~m}$ & $1941-1962$ & $\begin{array}{l}0.3 \mathrm{to} \\
0.6 \mathrm{~m} / \mathrm{yr}\end{array}$ & $1050 \mathrm{~m}$ & $\begin{array}{l}\text { No - Good bond } \\
\text { with ground. } \\
\text { Note: casing } \\
\text { above subsi- } \\
\text { dence zone in } \\
\text { tension. }\end{array}$ \\
\hline $\begin{array}{l}\text { Lake Maracaibo, } \\
\text { Venezuela }\end{array}$ & $\begin{array}{l}\text { 0il produced from } \\
\text { interbedded sands, hard } \\
\text { clays, silts (Miocene) }\end{array}$ & $3.3 \mathrm{~m}$ & $\begin{array}{l}1926-1954 \\
\text { continued } \\
\text { after but } \\
\text { at lower } \\
\text { rate }\end{array}$ & & & $\begin{array}{l}\text { No - casing } \\
\text { failed at } \\
\text { depth in pro- } \\
\text { duction strata }\end{array}$ \\
\hline Po Delta, Italy. & $\begin{array}{l}1000 \mathrm{~m} \text { (up to } 2000 \mathrm{~m} \text { ) } \\
\text { of relatively unconsol- } \\
\text { idated Quaternary } \\
\text { deposits; marine; san- } \\
\text { dy, highly calcareous } \\
\text { with some clay layers; } \\
\text { contains at least five } \\
\text { methane-bearing hori- } \\
\text { zons. }\end{array}$ & & $\begin{array}{l}1890-1950 \\
1950-1956\end{array}$ & $\mid \begin{array}{l}1-3 \mathrm{mn} / \mathrm{yr} \\
0.3 \mathrm{~mm} / \mathrm{yr}\end{array}$ & & $\begin{array}{l}\text { Yes - } 714 \mathrm{~m} \\
\text { deep well } \\
\text { filled with } \\
\text { concrete }\end{array}$ \\
\hline Osaka, Japan & $\begin{array}{l}\text { Ground water aquifers } \\
\text { containing clay zones }\end{array}$ & $\left|\begin{array}{c}0.3 \text { to } 0.6 \mathrm{~m} \\
1.3 \mathrm{~m} \\
2 \mathrm{~m}\end{array}\right|$ & $\begin{array}{l}1885-1928 \\
1928-1943 \\
1942-1951\end{array}$ & $\mid \begin{array}{l}1.5-13 \mathrm{~mm} / \mathrm{yr} \\
70-120 \mathrm{~mm} / \mathrm{yr}\end{array}$ & $\mid \begin{array}{l}40-80 \mathrm{~m} \\
\text { some to } \\
180 \mathrm{~m} \text { depth }\end{array}$ & $\begin{array}{l}\text { Yes - wells \& } \\
\text { buildings } \\
\text { founded in rock } \\
\text { have risen } \\
\text { bbove ground } \\
\text { surface. }\end{array}$ \\
\hline Mexico City & $\begin{array}{l}\text { Sand \& Gravel w inter- } \\
\text { beds of clayey silt } \\
\text { (andesitic detrius) } \\
\text { from } 50-60 \mathrm{~m} \text { to } 500 \mathrm{~m} \\
\text { comprise groundwater } \\
\text { aquifer; overlain by } \\
\text { fine grained lake de- } \\
\text { posits; volcanic ash } \\
\text { water-transported } \\
\text { sediments }\end{array}$ & 4 to $7.5 \mathrm{~m}$ & $\begin{array}{l}\text { to } 1959 \\
1898-1938 \\
1938-1948 \\
1948-1952 \\
1952-\end{array}$ & $\mid \begin{array}{l}20 \mathrm{~mm} / \mathrm{yr} \\
150 \mathrm{~mm} / \mathrm{yr} \\
300-500 \mathrm{~mm} / \mathrm{yr} \\
250 \mathrm{~mm} / \mathrm{yr}\end{array}$ & $\begin{array}{l}\text { mostly top } \\
100 \mathrm{~m}\end{array}$ & $\begin{array}{l}\text { Protrusion of } \\
\text { wells common }\end{array}$ \\
\hline Las Vegas & $\begin{array}{l}\text { Groundwater aquifer: } \\
\text { alluvial deposits } \\
\text { (late Tertiary \& Quat- } \\
\text { ernary confined by blue } \\
\text { clay (lacustrine) }\end{array}$ & $\begin{array}{l}0.1 \mathrm{~m} \\
0.350 \mathrm{~m} \\
0.66 \mathrm{~m}\end{array}$ & $\begin{array}{l}1935-1941 \\
1935-1950 \\
1935-1963\end{array}$ & & & $\begin{array}{l}\text { Protrusion as } \\
\text { much as } 1 \mathrm{~m}\end{array}$ \\
\hline Santa Clara Valley & $\begin{array}{l}\text { Groundwater aquifer: } \\
\text { unconsolidated alluvial } \\
\text { \& bay deposits (clay, } \\
\text { silt, sand, gravel) } \\
\text { (Pleistocene \& recent) } \\
\text { over semi-consolidated } \\
\text { Santa Clara Formation } \\
\text { over consolidated bed- } \\
\text { rock }\end{array}$ & $\begin{array}{l}1.2 \mathrm{~m} \\
1.5 \mathrm{~m} \\
3.3 \mathrm{~m}\end{array}$ & $\begin{array}{l}1912-1933 \\
1934-1960 \\
1912-1963 \\
1960-1963\end{array}$ & $0.23 \mathrm{~m} / \mathrm{yr}$ & $\begin{array}{l}300 \mathrm{~m} \\
\text { deep }\end{array}$ & $\begin{array}{l}\text { Protrusion as } \\
\text { much as } 1 \mathrm{~m}\end{array}$ \\
\hline
\end{tabular}




\begin{tabular}{|c|c|c|c|c|c|c|c|c|c|c|c|c|}
\hline INSTRUIENT & AVAILABILITY & OPERATING PRINCIPLE & DEPTH RAGGE & $\begin{array}{l}\text { RAAGE OF VERTICAL } \\
\text { DISPLACEMEETT }\end{array}$ & $\begin{array}{l}\text { MANUFACTURER' } \\
\text { SENSITIVITY }\end{array}$ & ACCURACY & $\begin{array}{l}\text { MAXIMUM DOWHYHOLE } \\
\text { TIEPREATUE: } \\
\text { RATED OR (EST- } \\
\text { MATED }\end{array}$ & 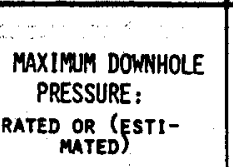 & $\begin{array}{l}\text { MATERIAL } \\
\text { COMPOSITION }\end{array}$ & $\begin{array}{l}\text { OPERATION AND INSTALLATION } \\
\text { (IMPORTANT FEATURES) }\end{array}$ & $\begin{array}{l}\text { MANTENAACE RE- } \\
\text { MUIREMENTS \& ESTI- } \\
\text { MATED SERVICE LIFE }\end{array}$ & $\begin{array}{l}\text { SELECTED } \\
\text { REFERENCES }\end{array}$ \\
\hline 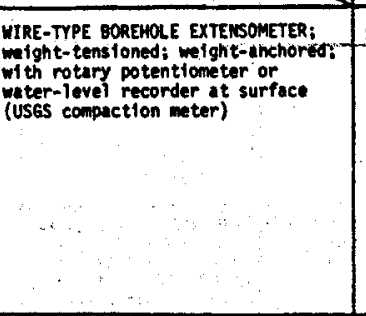 & \begin{tabular}{|l|} 
Shncoi user-fabricated \\
(A)
\end{tabular} & 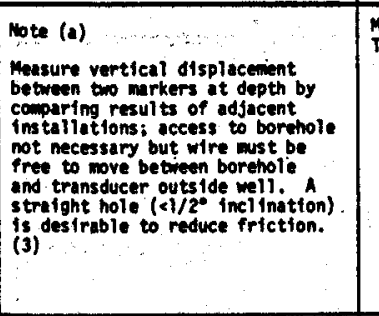 & Maximan: $6600 m$ i & 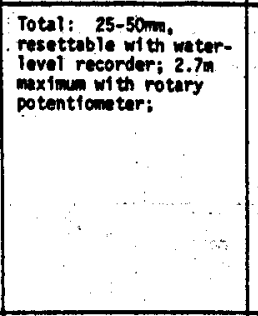 & $\begin{array}{l}50.03 \mathrm{~mm} \\
\end{array}$ & 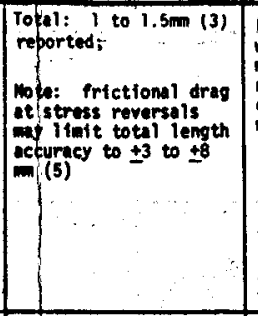 & 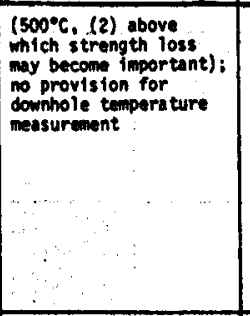 & mot applicable & 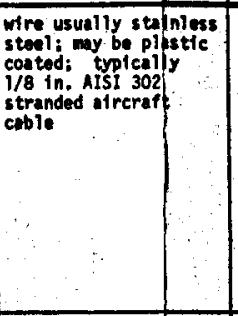 & 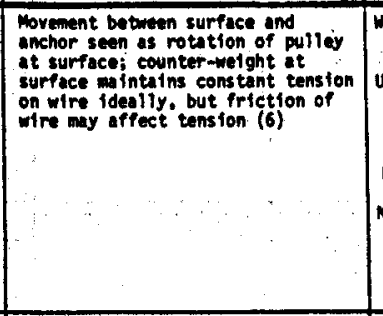 & 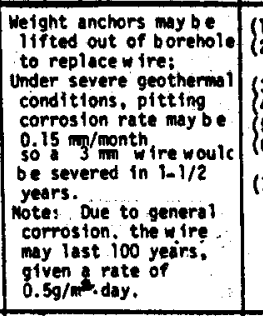 & 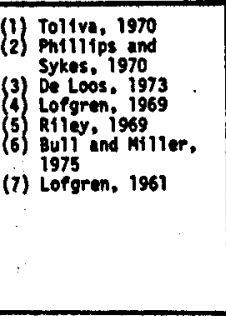 \\
\hline 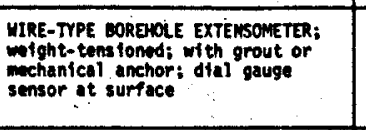 & 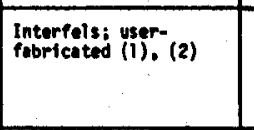 & ins above & Trpical: up to $100 \mathrm{~m}$ & 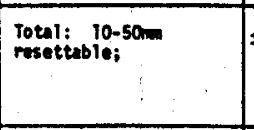 & 30,5 to $1.0 \mathrm{~m}$ & gall: 1.5 to $\mathrm{sm}$ & \begin{tabular}{|c|} 
as sobove \\
\end{tabular} & not applicible. & 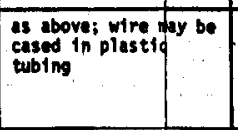 & 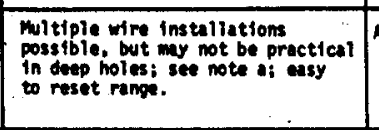 & 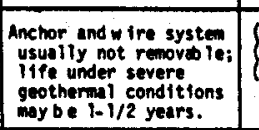 & 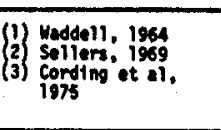 \\
\hline 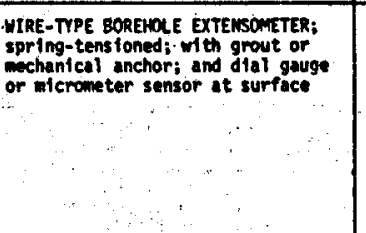 & $\begin{array}{l}\text { Terremetrics; Petor } \\
\text { sinth; serati }\end{array}$ & 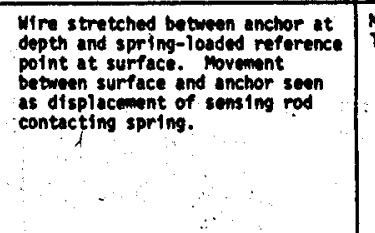 & $\begin{array}{l}\text { Maximan: } \\
\text { Typiteal: } \\
\text { T50-som: }\end{array}$ & 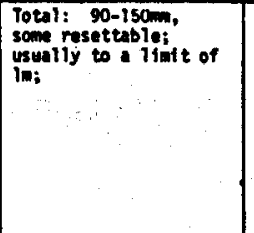 & $\begin{array}{c}0.03 \mathrm{~mm} \\
\vdots \\
\vdots \\
\vdots\end{array}$ & 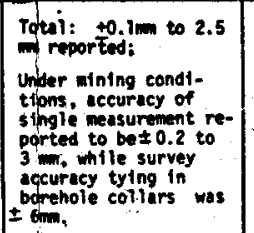 & os tovere. & not applictable & as soove & 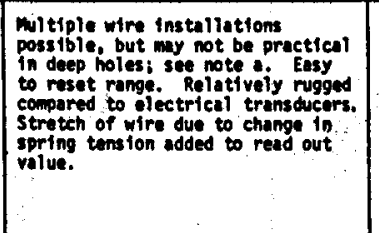 & as stovere & (1) Potts, 1964, \\
\hline 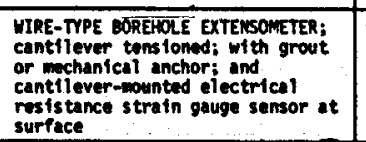 & Terrametrics & 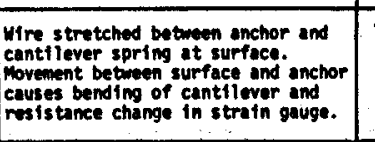 & Typteal: 150m & Total: $15 \mathrm{~m}$ rossttoble: & $\pm 0.01 \mathrm{~m}$ & 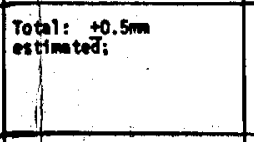 & as sobove & not applitecolis: & as sobve & 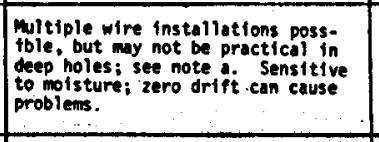 & 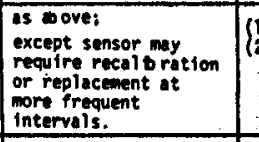 & 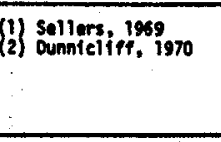 \\
\hline 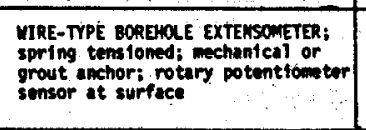 & Stinco & 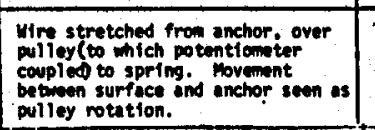 & Typtean: 100m assumed & $\begin{array}{l}\text { Tothl: som reast- } \\
\text { tutblo: }\end{array}$ & 5 & 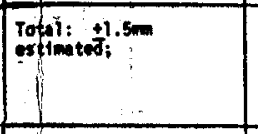 & as obove & not appilicable: & 33 cobove & as soove & os wover & (1) Dunntclifer, 1970 \\
\hline 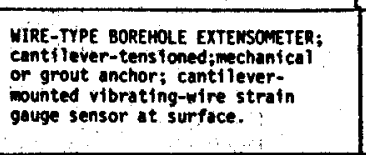 & \begin{tabular}{|l|} 
Telemenc \\
\end{tabular} & 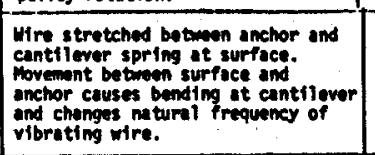 & Typiteal: $100 \mathrm{~m}$ & Total: 50 m & $\$ 0.01 \mathrm{~m}$ & 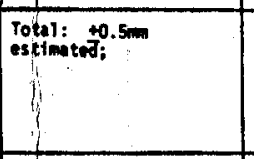 & as soove & not dopilicable & $\begin{array}{l}\text { sutniness staeel } \\
\text { wires }\end{array}$ & 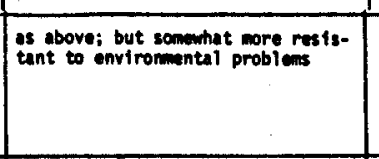 & as soovere & 1) Dunncliff, 1970 \\
\hline 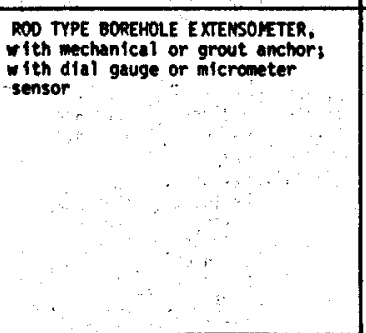 & 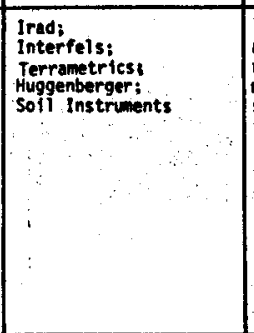 & 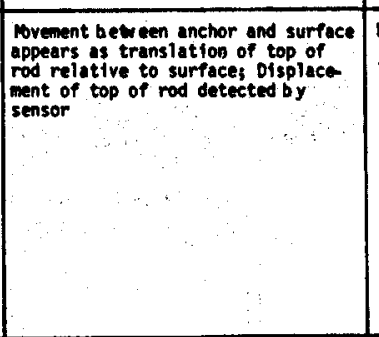 & 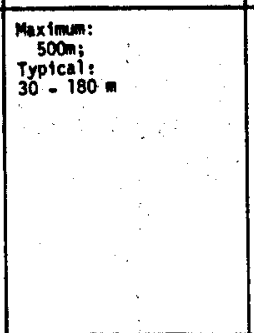 & 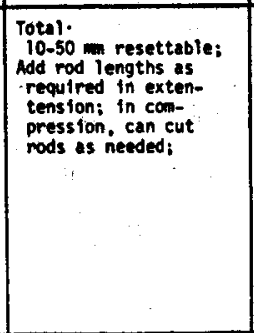 & $\pm 0.01 \mathrm{~m} \mathrm{to} \pm 0.03 \mathrm{~m}$ & 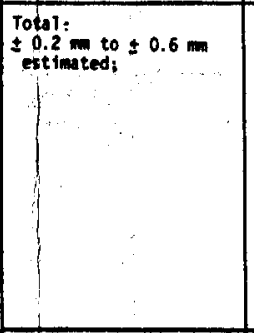 & as sove & not spplitcable & 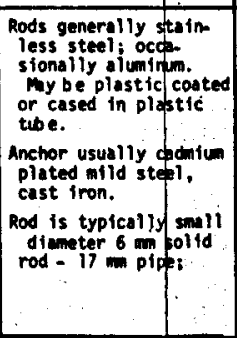 & 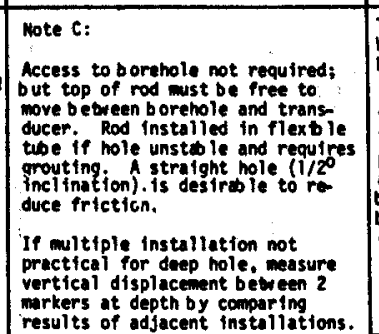 & 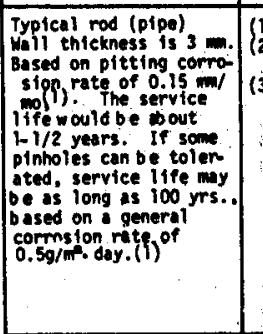 & 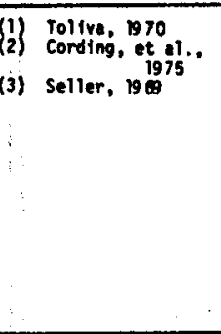 \\
\hline 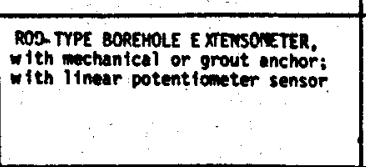 & 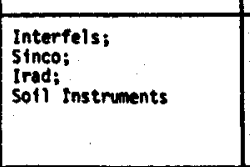 & os sovere & Trpical: $180 \mathrm{~m}$. & Total: $10-150 \mathrm{~m}$ & $\pm 0.01 \mathrm{~mm}$ to $0.1 \mathrm{~mm}$ & 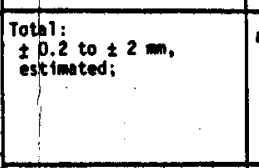 & los wove & not appl icable & as bove & \begin{tabular}{|l|} 
is sove \\
\end{tabular} & 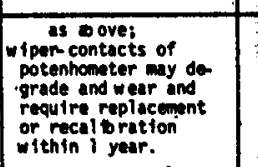 & \\
\hline 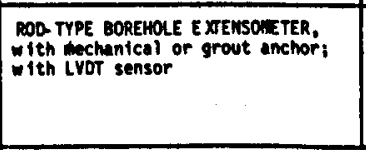 & Interfels & is sove & 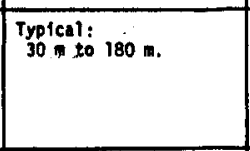 & Total: reseteble: & $\pm .01 \mathrm{~m} \mathrm{to} \pm 0.1 \mathrm{~m}$ & 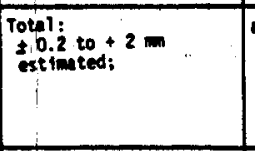 & es sove & not appllfeable & is oove & as sovere & 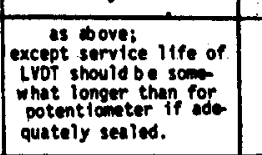 & \\
\hline 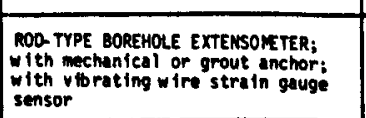 & Ma inak & as sove & 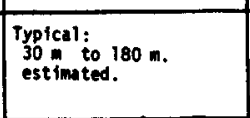 & 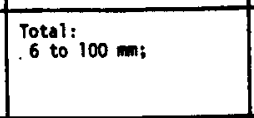 & $2.003 \mathrm{~m} \mathrm{tom} \pm .03 \mathrm{~mm}$ & 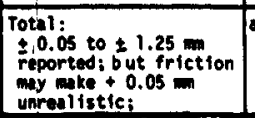 & as oove & \begin{tabular}{|l|} 
not applifcable \\
\end{tabular} & as bove & es wove & 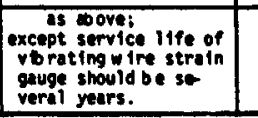 & \\
\hline
\end{tabular}


TABLE 2.11 CAPABILITIES OF EXISTING SUBSIDENCE MONITORINO INSTRUMENTS

\begin{tabular}{|c|c|c|c|c|c|c|c|c|c|c|c|c|c|}
\hline PROPERTIES & AVAILABILITY & OPERATING PRINCIPLE & DEPTH RANGE. & $\begin{array}{l}\text { RAAGE OF VERTICAL } \\
\text { DISPLACERESTT }\end{array}$ & $\begin{array}{l}\text { RANUFACTURER'S } \\
\text { SENSITIVITY }\end{array}$ & & JRACY & 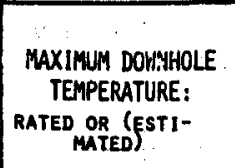 & $\begin{array}{l}\text { MXXIMUM DOWHHOLE } \\
\text { PESSUR: } \\
\text { RATED OR } \\
\text { MRTESTST- }\end{array}$ & $\begin{array}{l}\text { MATERIAL } \\
\text { COMPOSITION }\end{array}$ & $\begin{array}{l}\text { OPERATION AND INSTALLATION } \\
\text { (IMPORTANT GEATURES) }\end{array}$ & \begin{tabular}{|l|} 
MALNTENANCE RE- \\
OUIREMENTS \& ESTI- \\
MATED SERVICE LIFE
\end{tabular} & $\begin{array}{l}\text { SELECTED } \\
\text { REFEREMCES }\end{array}$ \\
\hline 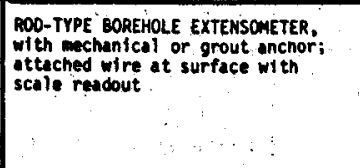 & User Fabricatted (1) & 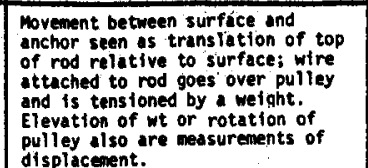 & Typical: $100 \mathrm{~m}$ & $1 \mathrm{~m}$. & $\pm 2.5 \mathrm{~mm}$ & Total: & estimated & is sobove & not applicable & as abovere & \begin{tabular}{|l|l|} 
as above \\
\end{tabular} & \begin{tabular}{|l|} 
as above \\
\end{tabular} & 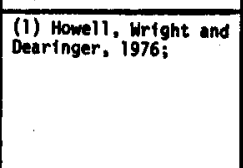 \\
\hline 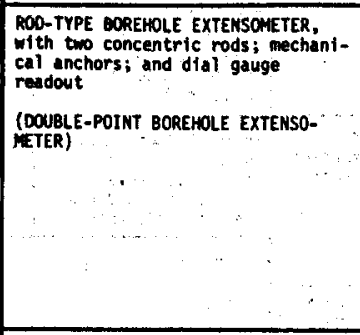 & 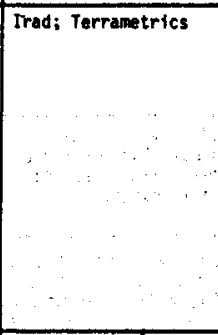 & 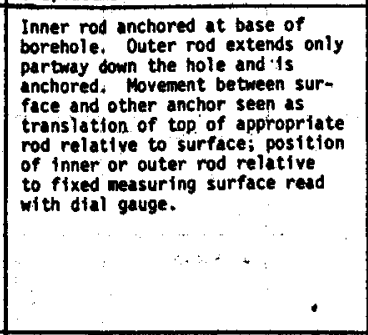 & $\mid$ & 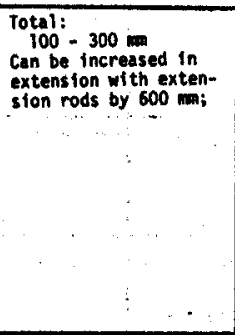 & $\begin{array}{l} \pm 0.01 \mathrm{~mm} \\
\end{array}$ & $\begin{array}{l}\text { Tototo: } \\
\pm 0.5 . \\
\pm .5\end{array}$ & nestimated & Oas bobve & not spplicabie & 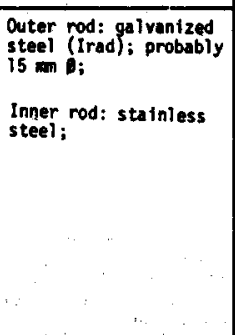 & 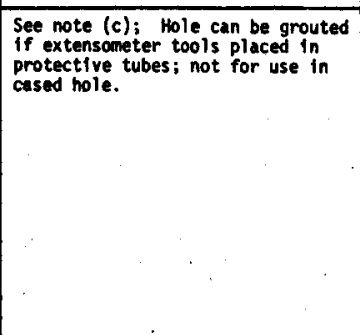 & 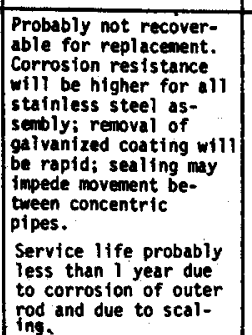 & \\
\hline 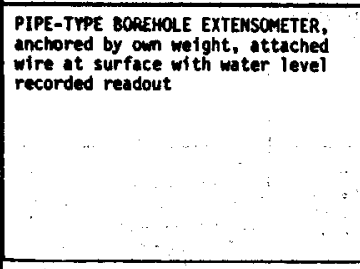 & $\begin{array}{l}\text { User Fobrtcated (1). } \\
\text { (2). (3) }\end{array}$ & 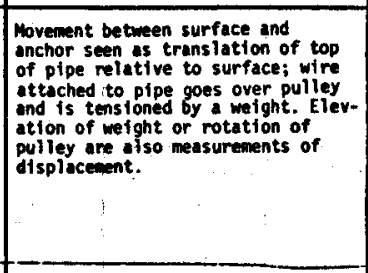 & 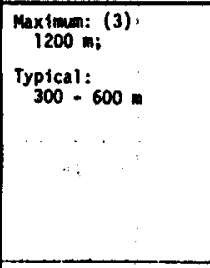 & 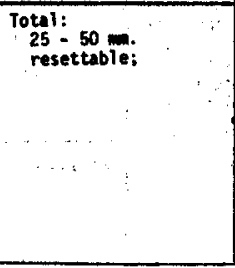 & 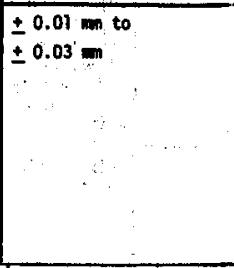 & 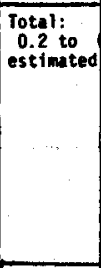 & & as above & not applicable & 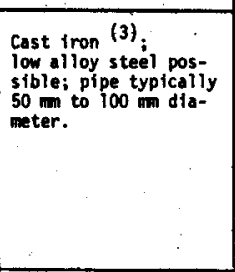 & 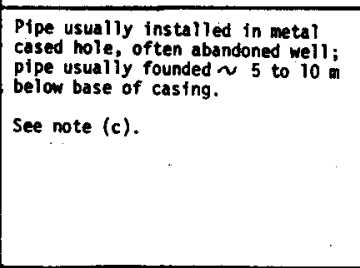 & 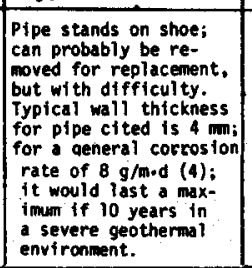 & 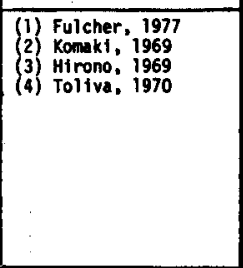 \\
\hline 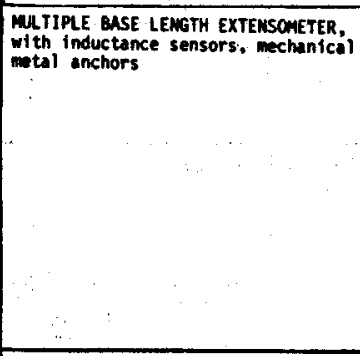 & Telemenc & 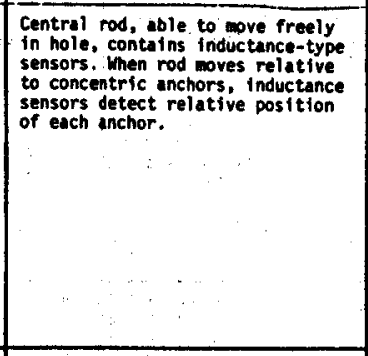 & 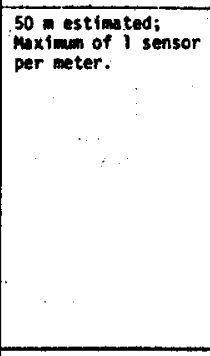 & naxian: & $0.01 \mathrm{~m}$ & Total and & $\begin{array}{l}\text { Interval: } \\
\text { clatined; }\end{array}$ & $50^{\circ} \mathrm{C}$ & 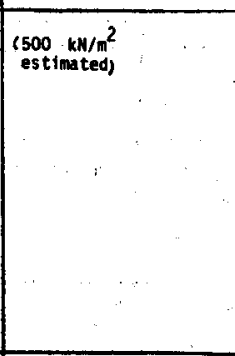 & $\begin{array}{l}\text { Probobaly stuinless } \\
\text { stees }\end{array}$ & 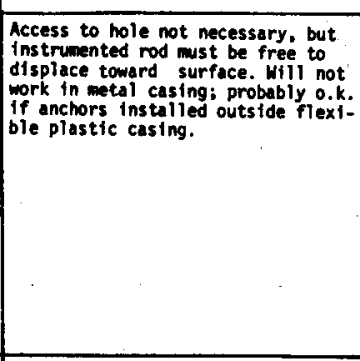 & 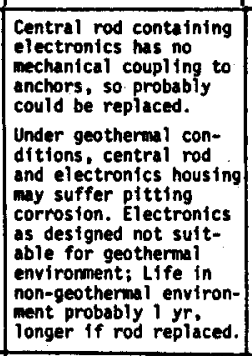 & \\
\hline 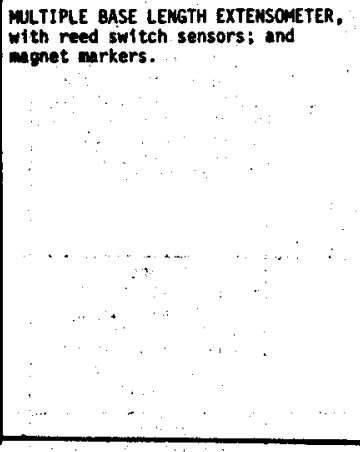 & 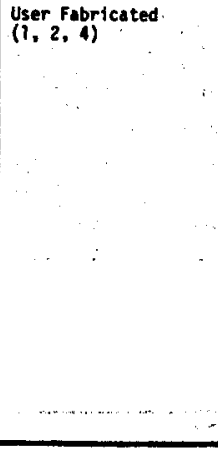 & 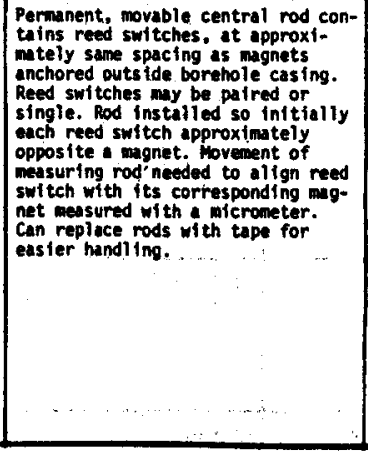 & $30-70 m$ & 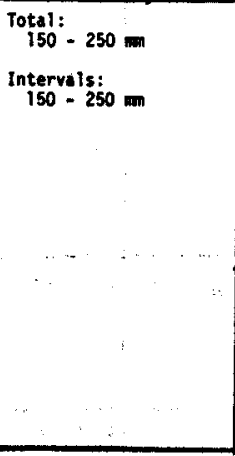 & $\pm 0.02 \mathrm{~mm}$ to & 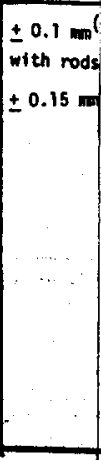 & 12) ${ }_{\text {reported }}$ & 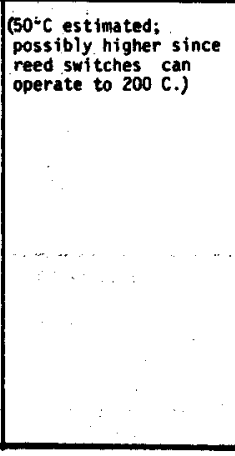 & 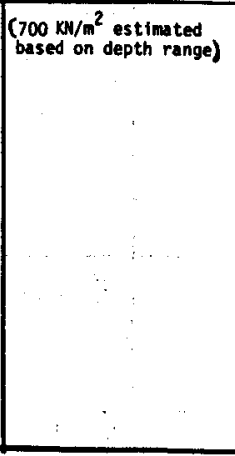 & 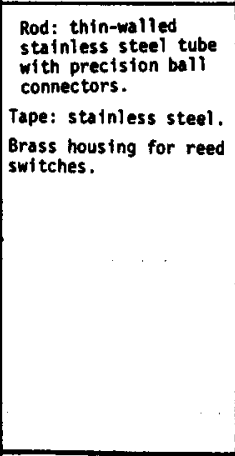 & 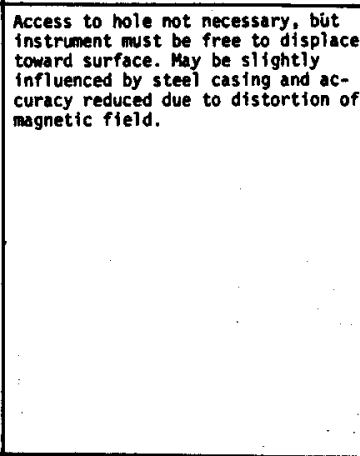 & 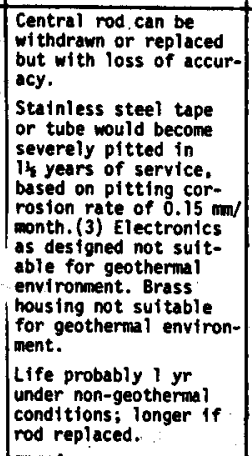 & 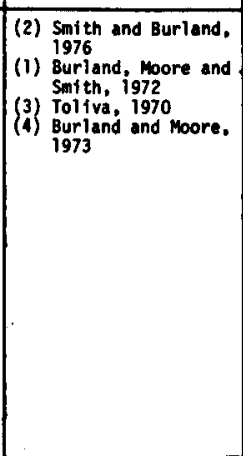 \\
\hline
\end{tabular}




\begin{tabular}{|c|c|c|c|c|c|c|c|c|c|c|c|c|c|}
\hline PROPERTIES & AVAILABILITY & OPERATING PRINCIPLE & DEPTH RAMGE & $\begin{array}{l}\text { RABGE OF VERTICAL } \\
\text { DISPLACERENT }\end{array}$ & $\begin{array}{l}\text { MAAUFACTURER'S } \\
\text { SENSITIVIT' }\end{array}$ & ACCURACY & 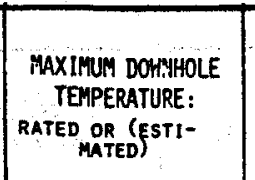 & 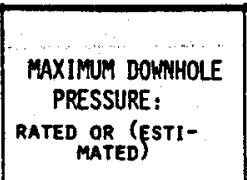 & $\begin{array}{l}\text { MATERIAL: } \\
\text { COMPOSITIOA }\end{array}$ & & $\begin{array}{l}\text { OPERAT FoN and INSTALLATION } \\
\text { (IMPOB́tant fEATURES) }\end{array}$ & \begin{tabular}{|l|} 
MAINTENAMCE RE- \\
OUIREMENTS \& ESTI- \\
MATED SERVICE LIFE
\end{tabular} & $\begin{array}{l}\text { SELECTED } \\
\text { REEEREMCES }\end{array}$ \\
\hline 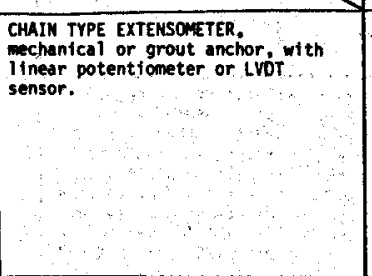 & $\begin{array}{l}\text { solit instruments; } \\
\text { Interfels }\end{array}$ & 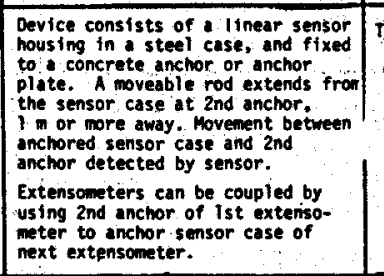 & 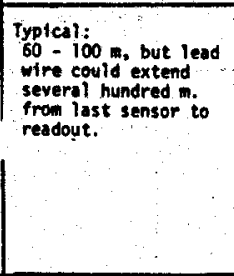 & 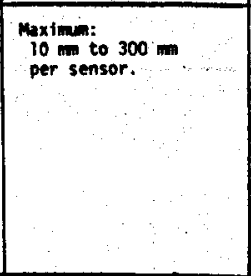 & $=0.1 .0 \mathrm{~mm}$ & 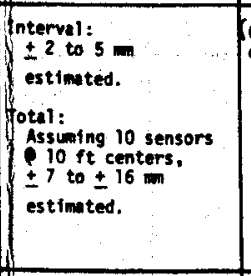 & 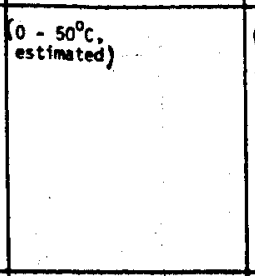 & 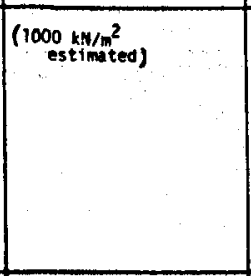 & \begin{tabular}{|l}
$\begin{array}{l}\text { Probobaty stati" } \\
\text { steel }\end{array}$ \\
\end{tabular} & & 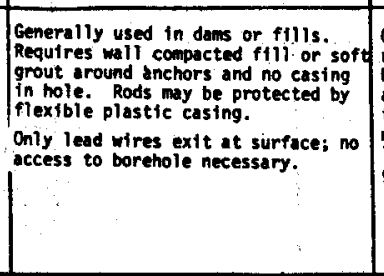 & 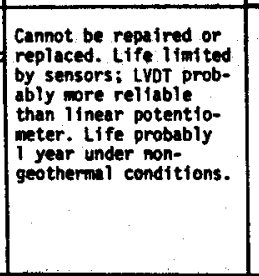 & \\
\hline 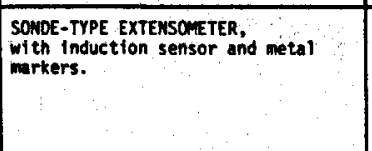 & 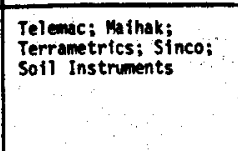 & 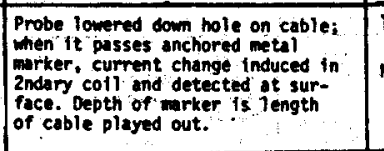 & 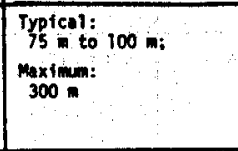 & 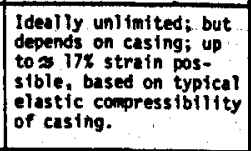 & $0.1 \mathrm{~m} \mathrm{to} \pm 1.5 \mathrm{~m}$ & 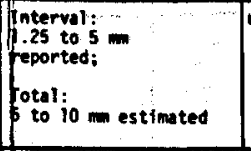 & up to $110^{\circ} \mathrm{C}$ & 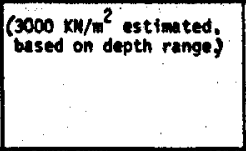 & 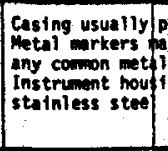 & $\begin{array}{l}\text { plastic. } \\
\text { yy be } \\
\text { ling } \\
\end{array}$ & 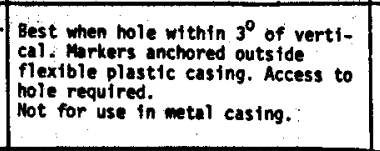 & 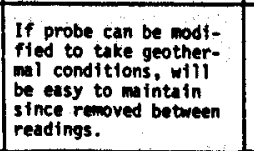 & \\
\hline 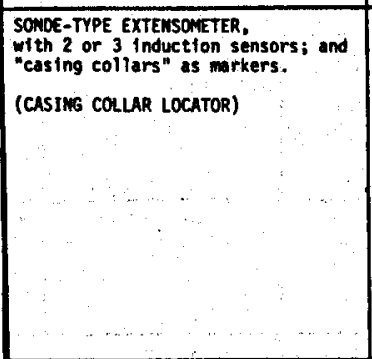 & 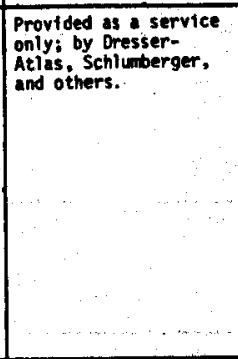 & 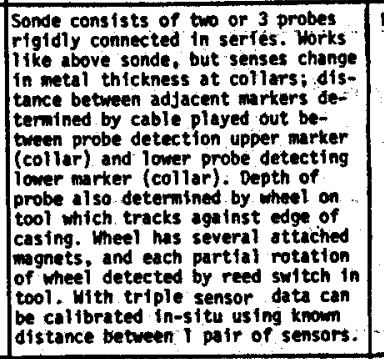 & $\operatorname{maximn:}$ & 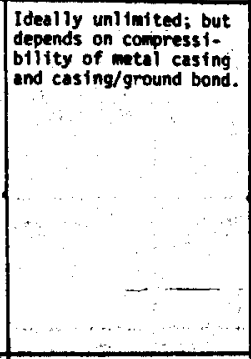 & 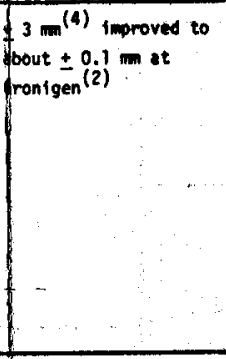 & 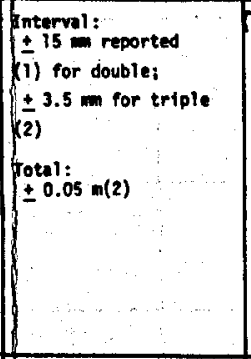 & 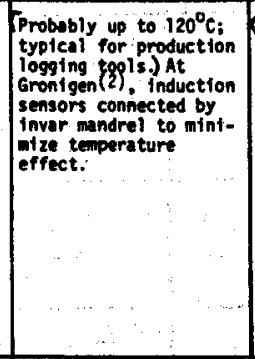 & 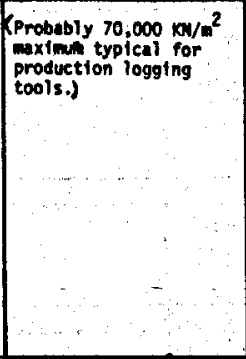 & 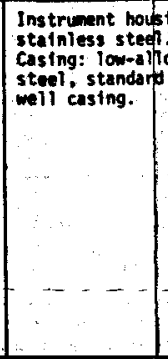 & & 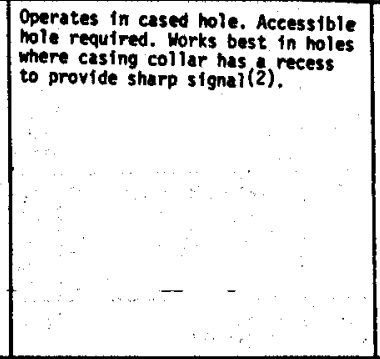 & \begin{tabular}{|l|l|} 
as above \\
\end{tabular} & 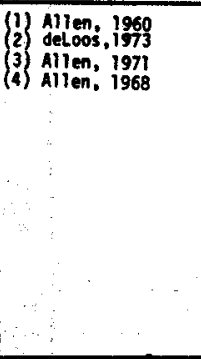 \\
\hline 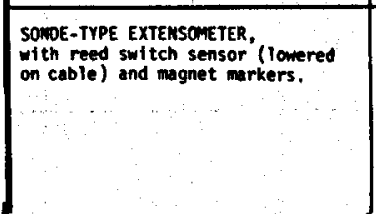 & $\begin{array}{l}\text { Soll Instruments; } \\
\text { Terra Technology; } \\
\text { ELE }\end{array}$ & 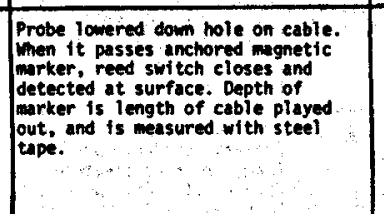 & $\begin{array}{l}\text { Trpoten!: } \\
\text { 100 : }\end{array}$ & 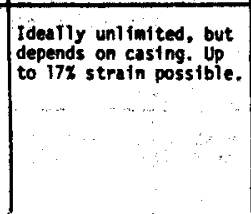 & $1-2 m^{(1)}$ & 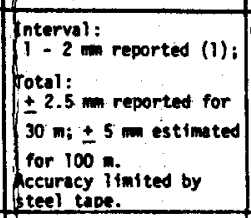 & 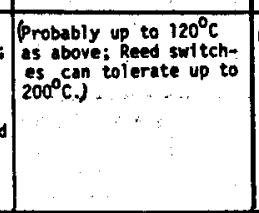 & 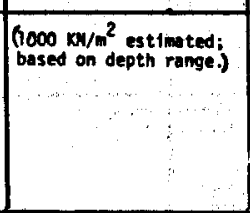 & 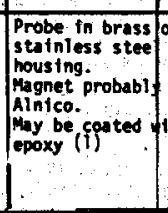 & & 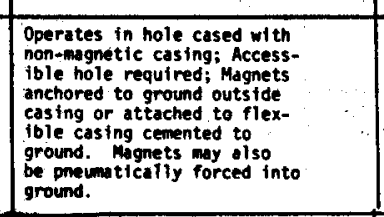 & as sobove & 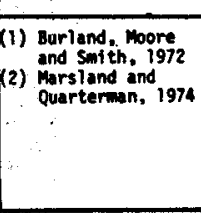 \\
\hline 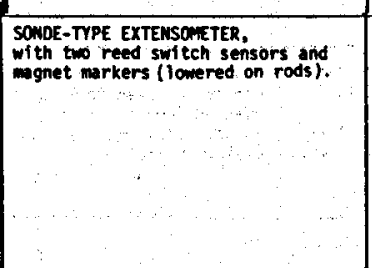 & soil instruments. & 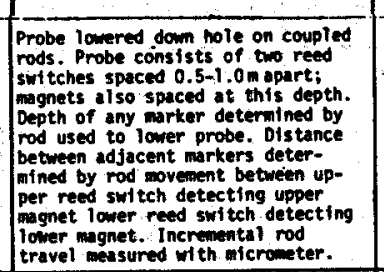 & 30 mestimated & $\begin{array}{l}\text { Tottl: } \\
\text { as dovere } \\
\text { interval: } \\
150-250 \mathrm{~m}\end{array}$ & $0.05=$ & 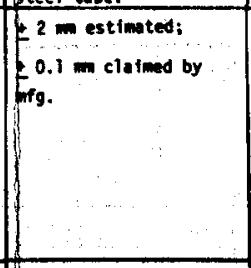 & 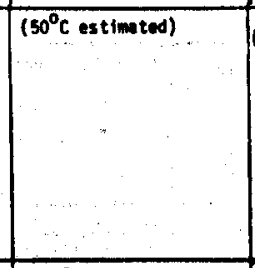 & 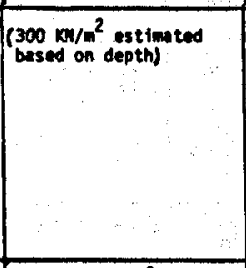 & 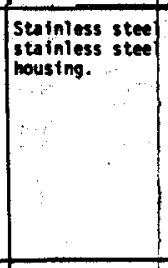 & rodsi & 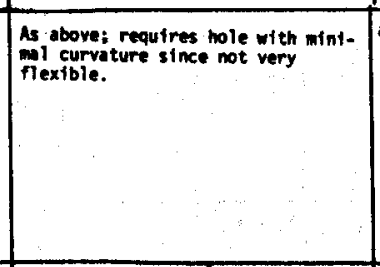 & os sobve & \\
\hline 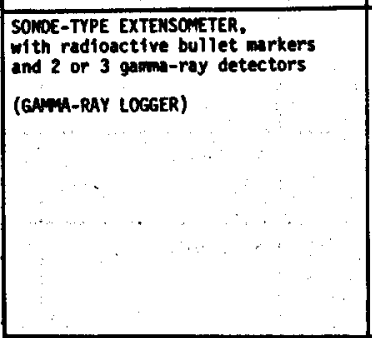 & 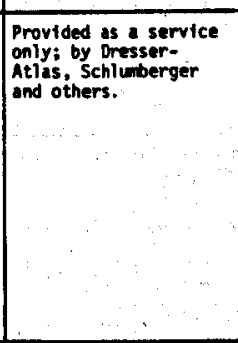 & 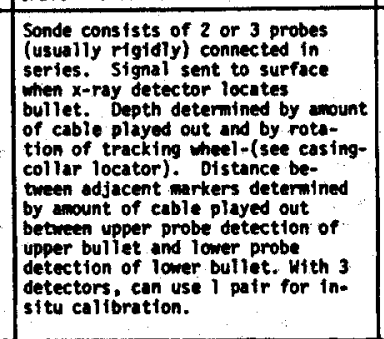 & $1500=$ & $\begin{array}{l}\text { Unnilitited because } \\
\text { bullets outstide casing } \\
\text { in formetion. }\end{array}$ & $0.1 \mathrm{~mm}$ & 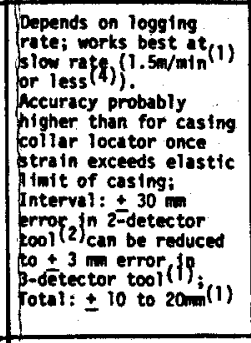 & 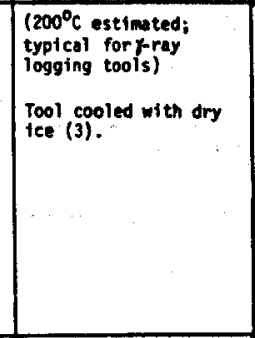 & 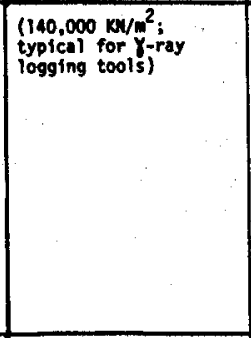 & 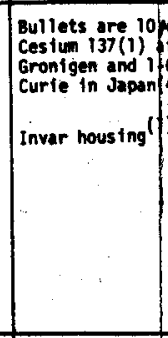 & $\begin{array}{l}\text { Curte } \\
6 \\
6 \\
6\end{array}$ & 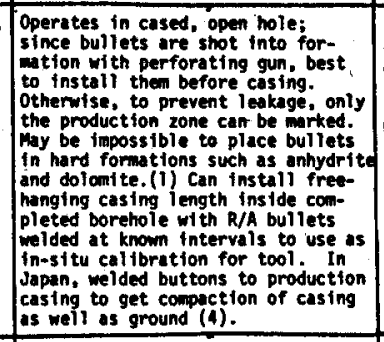 & 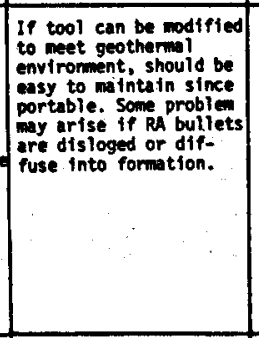 & 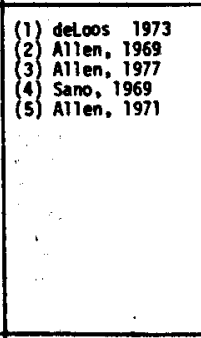 \\
\hline 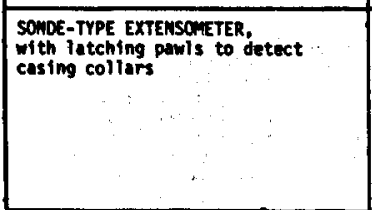 & 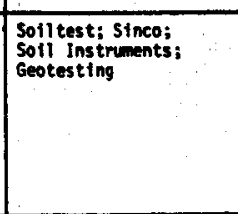 & 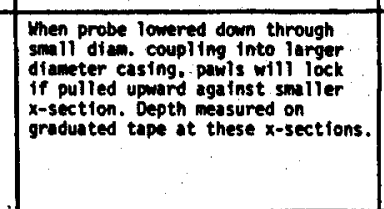 & \begin{tabular}{|l} 
Typical: \\
ioo an
\end{tabular} & 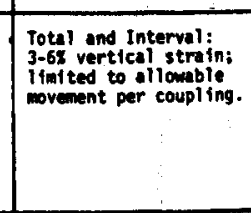 & $\neq 1$ to $\pm 3 \mathrm{~m}$ & 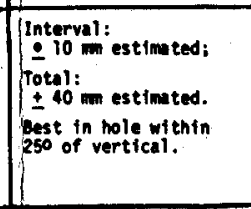 & (50 $0^{\circ} \mathrm{C}$ estimuted) & 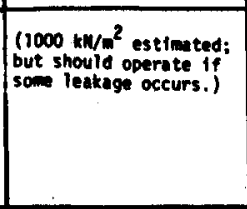 & 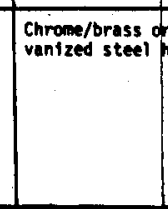 & pousting & 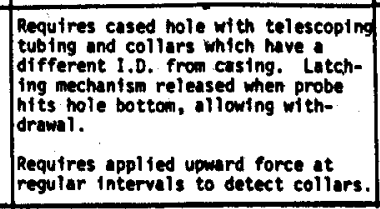 & 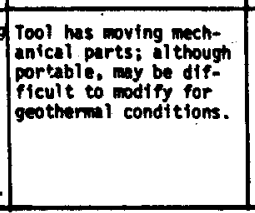 & \\
\hline
\end{tabular}




\begin{tabular}{|c|c|c|c|c|c|c|c|c|c|c|c|c|}
\hline IMSTRUEFTT PRogerties & Avallabilitit & OPERATIMG PRINCIPLE & DEPTH RANGE & 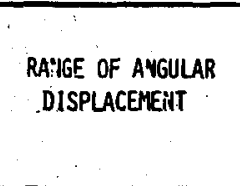 & 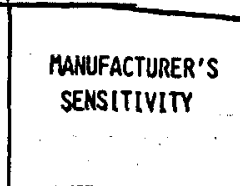 & Accuracy & 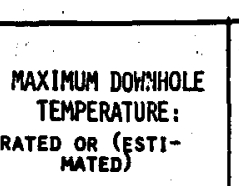 & 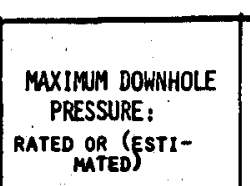 & $\begin{array}{l}\text { Rateriat } \\
\text { corposition }\end{array}$ & 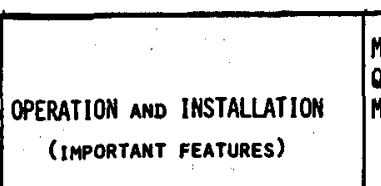 & 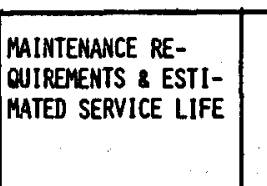 & $\begin{array}{l}\text { SELECEED } \\
\text { REFERECCSS }\end{array}$ \\
\hline 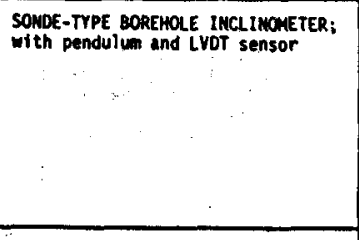 & 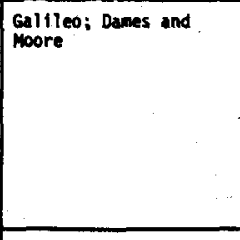 & 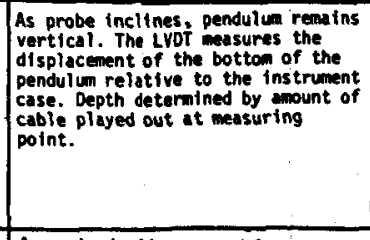 & 300 n estimaters & $12^{4+9}$ & $\pm 1 \min$ & 10 on ostinted & 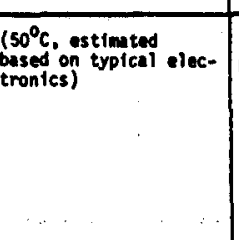 & 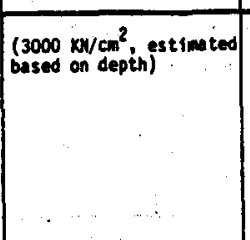 & 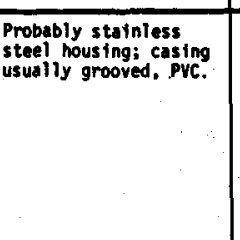 & 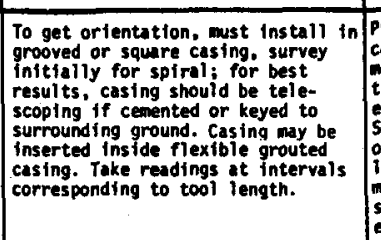 & 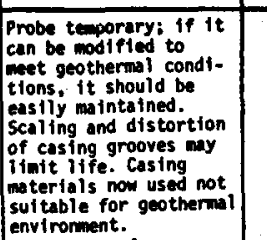 & \\
\hline 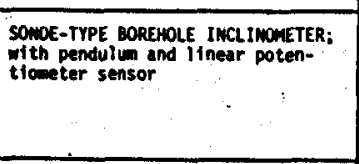 & sinico & 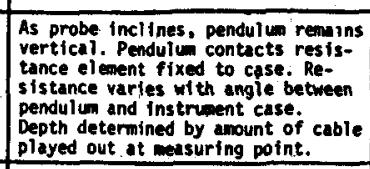 & 300. & 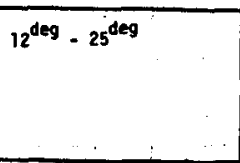 & $\pm 3 \min$ & $200 \sin$ & (ass soovere). & $1400 \mathrm{Nuv \textrm {C } ^ { 2 }}$ & 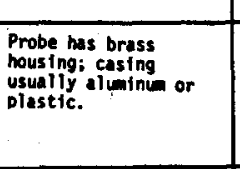 & is sobve & is soove & Wilison, 1962 \\
\hline 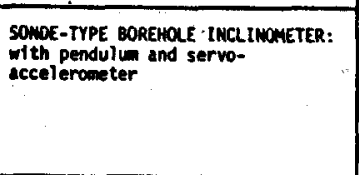 & 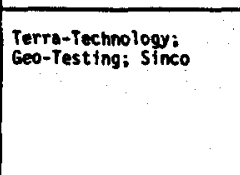 & 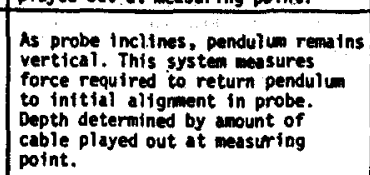 & 3000 & 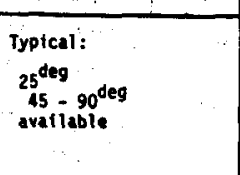 & $\sum 10 \mathrm{sec}$ & \pm 1 an resportes (1) & 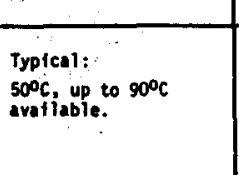 & 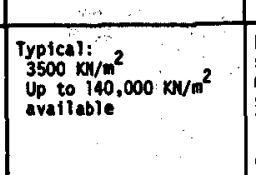 & 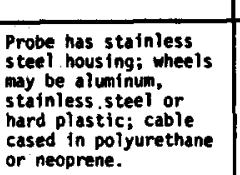 & is sobue & as sovere & 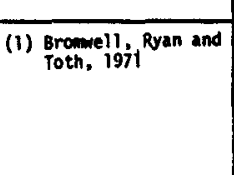 \\
\hline 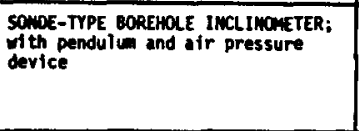 & Terratec, devel lopmente & 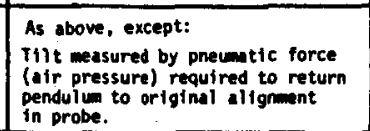 & 1000 estimestes & & $\pm 35 \mathrm{sec}$ & 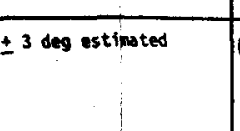 & 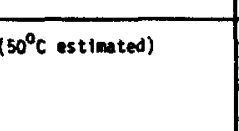 & \begin{tabular}{|c|}
$\left(1000 \mathrm{~km} / \mathrm{m}^{2}\right.$ estimstec) \\
\end{tabular} & 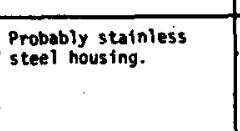 & sos bovere & as soover & Uumiteliff, 1970 \\
\hline 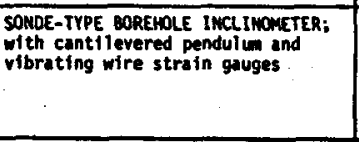 & 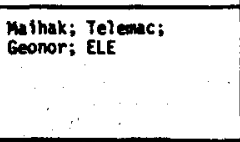 & 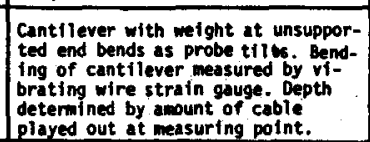 & 300 n estintinted & Tyitcal: & $230 \mathrm{sec}- \pm 2 \sin$ & \pm 3.004 tell repertest & 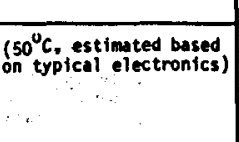 & 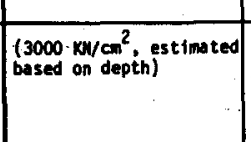 & 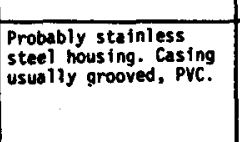 & as sovere & as abver & \\
\hline 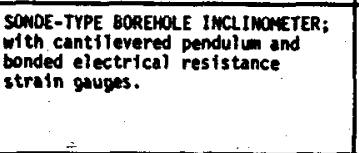 & 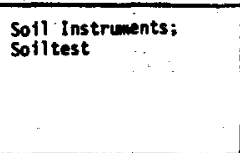 & 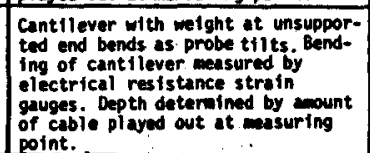 & 200. & 5 deeg to 30 des, & ₹ 15 sece to \pm 40 sec & 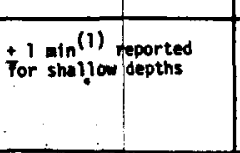 & (as sobver) & 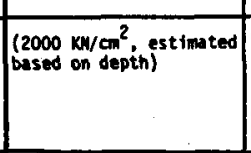 & 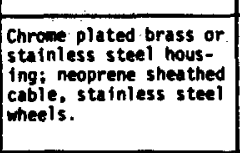 & ts s. sovere & as sovere & 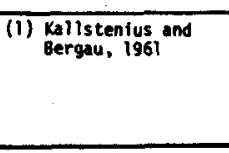 \\
\hline 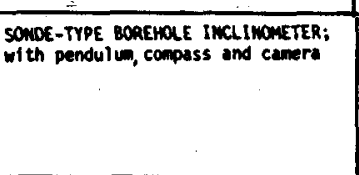 & 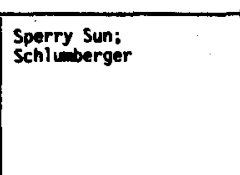 & 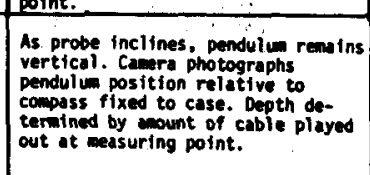 & 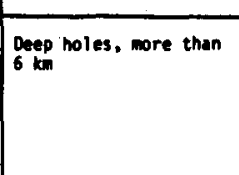 & $72^{\circ}$ to $130^{\circ}$ everilable & & & 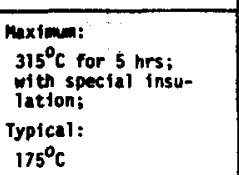 & $140,000 \mathrm{~mm} / \mathrm{m}^{2}$ & 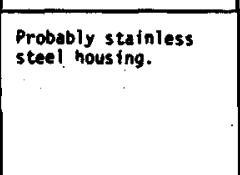 & 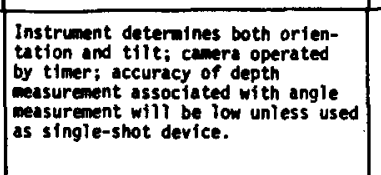 & 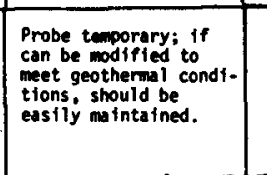 & \\
\hline 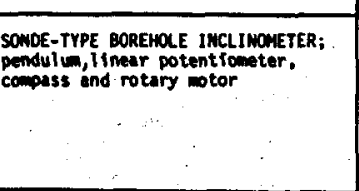 & Eestamen & 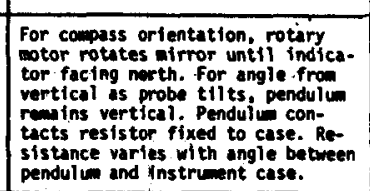 & $450 x-5000$ & 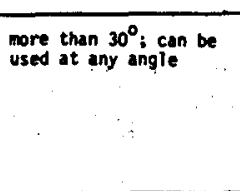 & 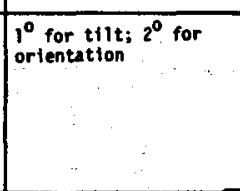 & & 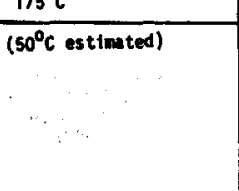 & $6350 \mathrm{NNV}^{2}$ & 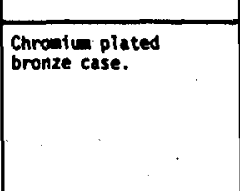 & 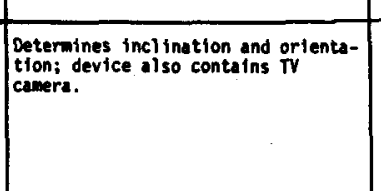 & as sover & \\
\hline 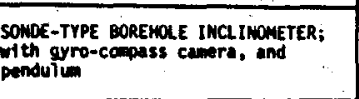 & 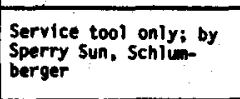 & 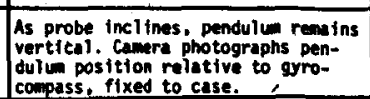 & 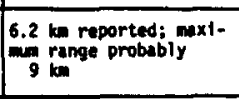 & $2^{0}$ to $00^{\circ}$ & & 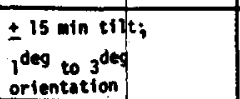 & 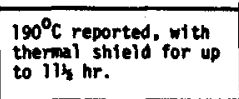 & 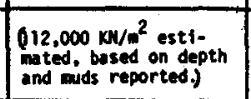 & Housing k-tonere & 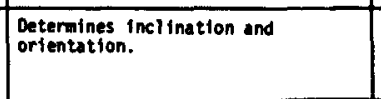 & as stove & OAl and cos Jun, 1971 \\
\hline 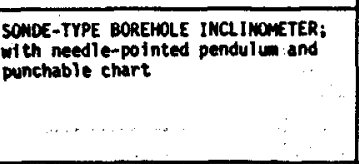 & Toreco & 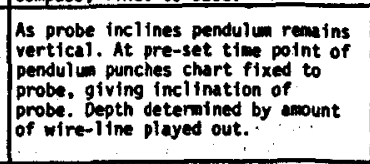 & 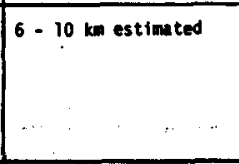 & $7,14,21$ def & 8 ain & 15 sin estitutes & 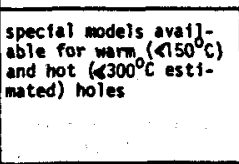 & 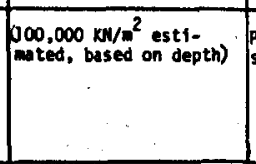 & 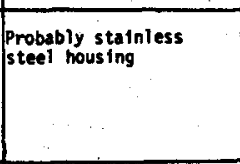 & 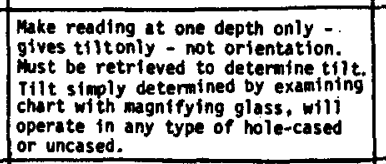 & as sovere & \\
\hline 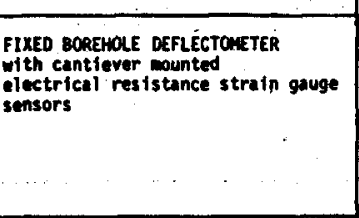 & \begin{tabular}{|l|l} 
Terramierics \\
\end{tabular} & 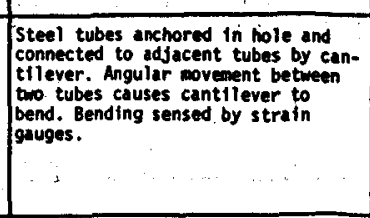 & 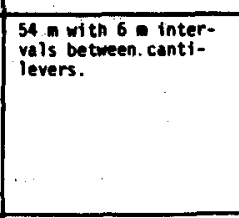 & & \pm 3 sec & $\pm 20 \mathrm{sec}$ & \begin{tabular}{|l|}
$50 \mathrm{C}$. \\
\end{tabular} & 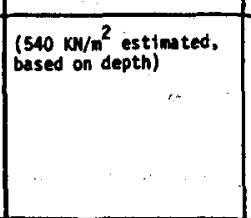 & 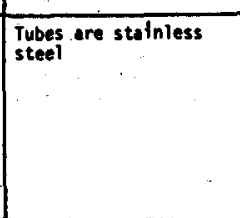 & 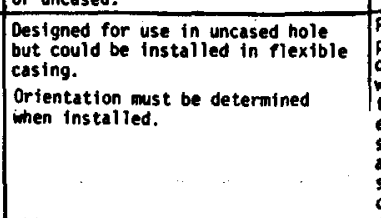 & 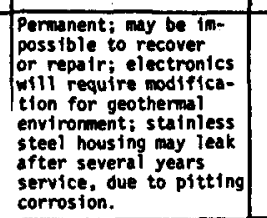 & \\
\hline 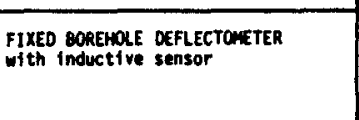 & \begin{tabular}{|l} 
Intererels \\
\end{tabular} & 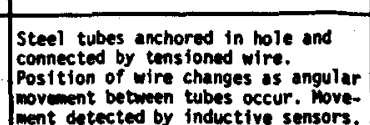 & 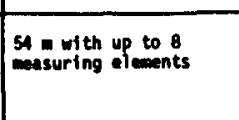 & 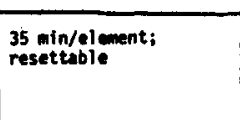 & \pm 2 to $\pm 20 \mathrm{sec}$ & II to 3 min estimuted & 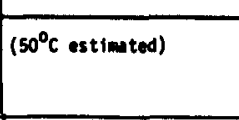 & 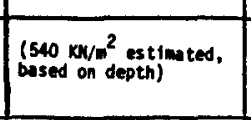 & 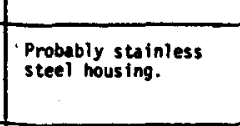 & as sobve & Ias sovere & \\
\hline 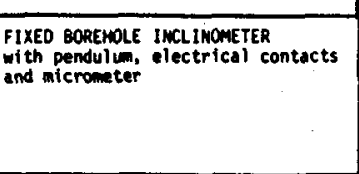 & S61 & 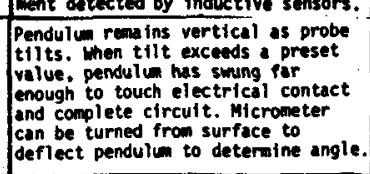 & 10 m estimuted & 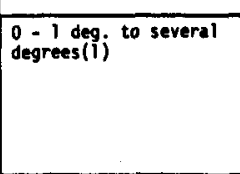 & \pm 6 ain to \pm 7 sec & $\begin{array}{l}2 \text { deg es } \\
\text { es }\end{array}$ & 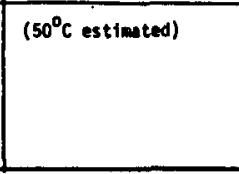 & 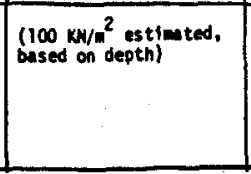 & 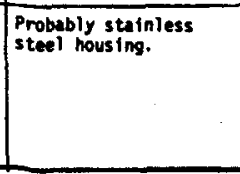 & 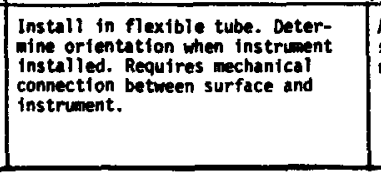 & 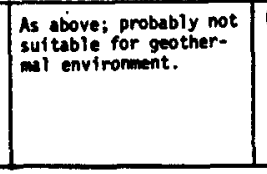 & 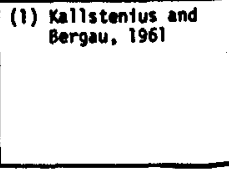 \\
\hline
\end{tabular}




\begin{tabular}{|c|c|c|c|}
\hline INSTRUMENT & 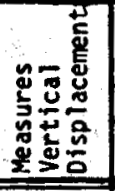 & 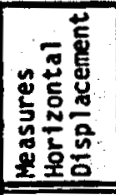 & DESCRIPTION OF INSTRUMENT \\
\hline $\begin{array}{l}\text { - Pipe Type Borehole Extensometer - Anchored by own Height, Attached } \\
\text { Wire at Surface with water level recorder readout. }\end{array}$ & $\sqrt{ }$ & & $\begin{array}{l}\text { A pipe inside a cased borehole is permanently anchared at the base of the } \\
\text { borehole and extends to the surface. The movement of the bottom of the } \\
\text { borehole relat tre to the surf ace is directiy determined from the movement } \\
\text { of the top of the plpe relative to a surface benchmark. }\end{array}$ \\
\hline $\begin{array}{l}\text { - Sonde-Type Borehole Extensometer - With } 2 \text { or } 3 \text { Induction Sensors and } \\
\text { "Casing Collars" as markers. } \\
\text { - Sonde-Type Borenole Extensometer with } 2 \text { or } 3 \text { Gamma-Ray Detectors and } \\
\text { Radioactive Bullet Markers. } \\
\text { - Sonde-Type Borehole Extensometer with Reed Switch Sensor (lowered on } \\
\text { cable) and Magnet Markers. }\end{array}$ & $\checkmark$ & & $\begin{array}{l}\text { A probe containing electronic sensors is lowered down a borehole. It detects } \\
\text { permanent markers placed at regular intervals along the borehole. The } \\
\text { markers may be magnets, casing collars or radioactive bullets. The distance } \\
\text { between markers may be determined by the amount of cabie played out or by } \\
\text { calibration of the strip chart record us ing the known spacing between probe } \\
\text { sensors if } 3 \text { or more sensors are used. The probe is retrieved after the } \\
\text { hole is surveyed. }\end{array}$ \\
\hline $\begin{array}{l}\text { - Sonde-Type Borehole Inclinometer with Pendulum and Servoaccelerometer } \\
\text { - Sonde-Type Borehole Inclinometer with Cantilevered Pendulum and } \\
\text { Vibrating Hire Strain Gauges } \\
\text { - Sonde-Type Borehole Inclinometer with Pendulum, Compass and Camera } \\
\text { - Sonde-Type Borehole Inclinometer with Pendulum and Punchable Chart } \\
\text { - Sonde-Type Borehole Inclinometer with Pendulum, Gyrocompass and } \\
\text { Camera }\end{array}$ & & $\begin{array}{c}2 \\
1 \\
1 \\
1 \\
1\end{array}$ & $\begin{array}{l}\text { A probe containing a electronic or mechanical sensor, whose signal is } \\
\text { proportional to the tiit of the probe, is lowered down a borehole. The } \\
\text { sensor may be a servaccelerometer, a strainn-gauged cant bevered pendulum } \\
\text { or a pendulum whose movement is recorded by a camera or punchable chart. } \\
\text { The depth of the probe is the length of cable played out. Horizontal } \\
\text { deflections. are calculated from the cont inuous tilt prof ile with depth. } \\
\text { The probe is retrieved after the hole is surveyed. }\end{array}$ \\
\hline $\begin{array}{l}\text { - Fixed Borehole Deflectometer with Cantilever Mounted Electrical } \\
\text { Resistance Strain Gauge Sensors }\end{array}$ & & $\checkmark$ & $\begin{array}{l}\text { A permanent instrument consisting of two tubes, hinged by } 2 \text { strain-gauged } \\
\text { cantilevers at the junct ion. Angular movement between the two tubes bends } \\
\text { the cant ilever. The strain in the cant ilever is measured electronically } \\
\text { from the surface. Horizontal deflect tons are calculated from the cont inuous } \\
\text { tilt profile provided by a series of these devices. }\end{array}$ \\
\hline
\end{tabular}

1 Mote: Refer to Interim Report $n$ for more information on these instruments 
TABLE. 2.13

INSULATION SUITABLE FOR GEOTHERMAL TEMPERATURES

(Malloy, 1969; Barron, 1966)

\begin{tabular}{|c|c|c|c|c|}
\hline & Insulation & $\begin{array}{l}\text { Range of } \mathrm{k} \\
\text { at } 93^{\circ} \mathrm{C} \\
\left(\mathrm{W} / \mathrm{m}^{\circ} \mathrm{C}\right) \\
\end{array}$ & $\begin{array}{l}\text { Range of } \mathrm{k} \\
\text { at } 315^{\circ} \mathrm{C} \\
\left(\mathrm{W} / \mathrm{m}^{\circ} \mathrm{C}\right) \\
\end{array}$ & Comments \\
\hline 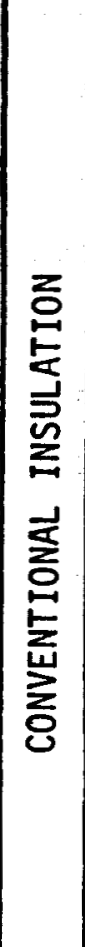 & $\begin{array}{l}\text { Asbestos Fibers } \\
\text { Calcium Silicate } \\
\text { Diatomaceous Silic } \\
\text { Cellular Glass } \\
\text { Glass Fibers } \\
\text { Magnesium Carbonate } \\
\text { Mineral Fiber } \\
\text { Expanded Perlite } \\
\text { Silica (and Alumina } \\
\text { Fibers }\end{array}$ & $\begin{array}{l}0.047-0.064 \\
0.047-0.065 \\
0.046-0.224 \\
0.060 \\
0.036-0.076 \\
0.050-0.065 \\
0.037-0.068 \\
0.045-0.051\end{array}$ & $\begin{array}{l}0.073-0.095 \\
0.073-0.088 \\
0.068-0.118 \\
0.109 \\
0.059 \\
0.065-0.097 \\
0.077 \\
0.051-0.087\end{array}$ & $\begin{array}{l}\text { With binders; without } \\
\text { binders, but woven, etc. } \\
\text { With binders and loose } \\
\text { With binders; without } \\
\text { binders but woven, etc. } \\
\text { With asbestos filler } \\
\text { With binders; without } \\
\text { binders but woven, etc; } \\
\text { loose } \\
\text { With binder, loose } \\
\text { Woven and other without } \\
\text { binders; loose }\end{array}$ \\
\hline 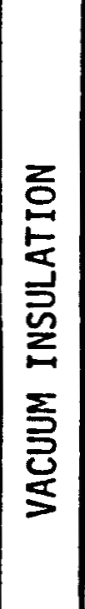 & $\begin{array}{l}\text { Multilayer Vacuum } \\
\text { Insulation }\end{array}$ & \multicolumn{2}{|c|}{$\begin{array}{l}0.0073 \text { between } 100^{\circ} \mathrm{C} \\
\text { and } 300^{\circ} \mathrm{C} \text { (calculated) } \\
0.0004 \text { between } 100^{\circ} \mathrm{C} \\
\text { and } 300^{\circ} \mathrm{C} \text { (calculated) }\end{array}$} & $\begin{array}{l}\text { Two concentric cylinders; } \\
\text { space between evacuated } \\
\text { to } 10^{-4} \mathrm{~mm} \mathrm{Hg;} \mathrm{100mm \phi ,} \mathrm{Im} \\
\text { long external dimensions; } \\
12.5 \mathrm{~mm} \text { gap between concentric } \\
\text { cylinders } \\
\text { Aluminum foil at } 2 \text { layers/ } \\
\text { mm; fiberglass spacer } \\
\text { material; } 10^{-4} \mathrm{~mm} \mathrm{Hg} \\
\text { pressure between foil } \\
\text { layers. }\end{array}$ \\
\hline
\end{tabular}

Note: Binders are inorganic and organic materials used to hold fibrous and granular insulation together. 
TABLE 2.14 THERMAL EXPANSION CHARACTERISTICS OF SELECTED MATERIALS

\begin{tabular}{|lc|}
\hline \multicolumn{1}{|c}{ Material } & $\begin{array}{c}\text { Coefficient of Expansion at } 20^{\circ} \mathrm{C} \\
\left(\mathrm{mm} / \mathrm{mm} / \mathrm{O}^{\circ}\right)\end{array}$ \\
\hline Aluminum & $20-22 \times 10^{-6}$ \\
Low Carbon Steel & $11-12 \times 10^{-6}$ \\
Invar & $1.4 \times 10^{-6}$ \\
Copper & $16 \times 10^{-6}$ \\
Concrete & $13 \times 10^{-6}$ \\
Vulcanized Rubber & $81 \times 10^{-6}$ \\
Nylon & $99 \times 10^{-6}$ \\
Glass (Silica) & $0.5 \times 10^{-6}$ \\
Calcite & $-6 \times 10^{-6}$ one axis; \\
& $25 \times 10^{-6}$ other axis \\
\hline
\end{tabular}

(after Van Vlack, 1964; INCO, 1968)

Average $\alpha_{L}$

For

Alloy Name \% Nickel Temperature Range

Invar

42-Nickel

Iron Alloy

49-Nicke1

Iron Alloy

$36 \quad 1.4-1.6 \times 10^{-6} \mathrm{~mm} / \mathrm{mm}^{\circ} \mathrm{C}$

40-42

$4.8-5.6 \times 10^{-6} \mathrm{~mm} / \mathrm{mm}^{\circ} \mathrm{C}$

$47-50$

$9.1-9.9 \times 10^{-6} \mathrm{~mm} / \mathrm{mm}^{\circ} \mathrm{C}$

Carbon Steel 0
$12.8 \times 10^{-6} \mathrm{~mm} / \mathrm{mm}^{\circ} \mathrm{C}$
Range of Temperatures Over Which $\alpha_{1}$ Nearly Constant

$-70^{\circ} \mathrm{C}$ to $120^{\circ} \mathrm{C}$ $40^{\circ} \mathrm{C}$ to $230^{\circ} \mathrm{C}$ $0^{\circ} \mathrm{C}$ to $-400^{\circ} \mathrm{C}$ gradually increases over range 0 to $300^{\circ} \mathrm{C}$

(Carpenter Steel Co., 1977; Inco, 1949) 


\section{(a) ELASTIC MODULUS}

The change in the modulus of elasticity due to temperature change is given by: $\Delta E=a_{M} \cdot E \cdot \Delta T$

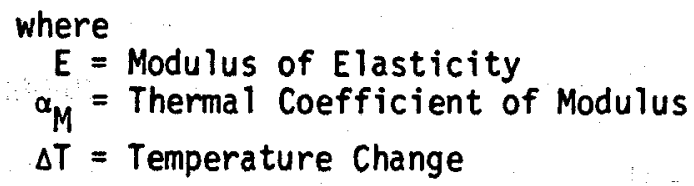

Typical values of the thermal coefficient of modulus $\alpha_{M}$ are given below.

\begin{tabular}{|ll|}
\hline \multicolumn{1}{|c|}{ Material } & \multicolumn{1}{c}{$\alpha_{M}\left(0-150^{\circ} \mathrm{C}\right)$} \\
\hline $\begin{array}{l}\text { Controlled Modulus Alloy } \\
\text { (Iso-elastic, Elinvar, Ni-Span-C) }\end{array}$ & -36 to $27 \times 10^{-6} /{ }^{\circ} \mathrm{C}$ \\
Spring Steel & $-342 \times 10^{-6},{ }^{\circ} \mathrm{C}$ \\
Type 316 Stainless Steel & $-400 \times 10^{-6},{ }^{\circ} \mathrm{C}$ \\
\hline
\end{tabular}

(Inco, 1963; John Chatilion \& Sons, 1978)

Note:

The controlled modulus alloys have a relatively small and constant thermal coefficient of modulus to $150^{\circ} \mathrm{C}$ (Inco, 1949). Age-hardening increases the temperature range (Inco, 1949), but data on the value of $\alpha_{M}$ above $150^{\circ} \mathrm{C}$ are not readily available.

(b) ELECTRICAL RESISTANCE

The change in electrical resistance due to temperature is given by: $\Delta R=\alpha_{R} \cdot \Delta T \cdot R$

$$
\begin{aligned}
\text { where } & \\
R & =\text { Electrical Resistance } \\
\alpha_{R} & =\text { Thermal Coefficient of Resistance } \\
\Delta T & =\text { Temperature Change }
\end{aligned}
$$

\begin{tabular}{|c|c|}
\hline Conductor & $\alpha_{R}\left(\Delta \Omega / \Omega-{ }^{\circ} \mathrm{C}\right.$ near $\left.20^{\circ} \mathrm{C}\right)$ \\
\hline Aluminum & 0.004 \\
\hline Carbon & -0.0003 \\
\hline Constantan $\begin{array}{r}(55 \% \mathrm{Cu} \\
45 \% \mathrm{Ni})\end{array}$ & o (average) \\
\hline Copper & 0.004 \\
\hline Manganin $\begin{array}{l}(84 \% \mathrm{CU} ; \\
12 \% \mathrm{Mn}, 4 \% \mathrm{Ni})\end{array}$ & \% (average) \\
\hline $\begin{array}{c}\text { Evanohm }(74 \% \mathrm{Ni} ; \\
20 \% \mathrm{Cr} ; 3 \% \mathrm{Al} \\
3 \% \mathrm{Fe})\end{array}$ & $\begin{array}{l}-0 \text { (average) }\left(\text { to } 250^{\circ} \mathrm{C}\right) \\
\text {; Inco, 1968) }\end{array}$ \\
\hline
\end{tabular}
Typical values of the thermal coefficient of electrical resistance $\alpha_{R}$
are given below. 
TABLE 2.16

TEMPERATURE LIMITS OF MECHANICAL SEALS

Temperature limits of common seals are:

$\begin{array}{ll}\text { Neoprene } & 135^{\circ} \mathrm{C} \\ \text { Viton } & 190^{\circ} \mathrm{C} \\ \text { PTFE } & 245^{\circ} \mathrm{C} \\ \text { Asbestos } & 345^{\circ} \mathrm{C} \\ \text { Nitinol with Composites } & 400^{\circ} \mathrm{C} \\ \text { Grafoil } & 400^{\circ} \mathrm{C} \\ \text { Metal to Metal } & 480^{\circ} \mathrm{C}\end{array}$

(Griffith, 1978; Baker, et al, 1975b) 
SOME RECOMMENDED MATERIALS AND TECHNIQUES

FOR GEOTHERMAL INSTRUMENTATION

ITEM

NO.

1

2

3

4

5

6

7
MATERIAL OR

TECHNIQUE

AL-6X or Type 316, Stainless Steel

Hastelloy-C or Inconel 625, Nickel Alloys

Cathodic Protection with impressed current

Chromium/Nickel or Aluminum Coatings

Inhibitors for $\mathrm{pH}$ control and oxygen removal; film-forming amine inhibitors

Design to minimize effect of scaling on instrument

Multi-layer foil vacuum insulation

Thermoelectric cooling or "special" mechanical heat pump cooling

40-50\% Nickel/Iron Alloy

Temperature-Invariant Reference Bar

Isoelastic, N-Span-C, Elinvar.

Evanohm, controlled resistance alloys

Use of two strain gauges-one strained and one unstrained

Heat treatment of strain gauges

Metal-to-metal seals

Metal sheathed, 4-wire, butt welded electrical cable with strain member

3 or more sensors per extensometer probe

Conversion to frequency or on/off signal or use of downhole recorders

Gyroscope
APPLICATION

To construct instrument housing for temporary probes.

To construct instrument housing for permanent downhole instruments.

To protect well casings used as part of subsidencemonitoring system; to protect permanent downhole instruments.

To extend life of metal parts exposed to well environment; use only with recognition of implications of coating falure

To reduce the rate of corrosion due to well fluids of of well casing and permanent downhole instruments.

To prevent probable build-up of calcite and silicá scale from rendering instrument inoperable.

To reduce heat flow into instrument capsule. Use inside pressure-resistant housing.

To remove heat from instrument capsule to maintain temperature at $100^{\circ} \mathrm{C}$ for a minimum of 72 hours; this allows for slow accurate logging with available electronics.

To construct internal instrument parts to reduce temperature effect on length.

To support internal instrument parts to reduce temperature effect on length.

To construct springs, cantilevers and tensioned members to reduce temperature effect on elastic properties and to reduce inelastic behavior at high temperature.

To construct precision resistors to minimize reversible electrical resistance change with temperature.

To compensate for temperature effects on resistance (both reversible and annealing-type changes) and on length.

To reduce cumulative resistance changes in strain gauges due to gradual annealing at geothermal temperatures.

To minimize leakage into the instrument at geothermal pressures and temperatures.

To design and construct electrical cable to withstand geothermal fluid pressures and temperatures.

To make measurement of distance between markers independent of cable stretch and minimally affected by tool bounce.

To eliminate inaccuracies due to signal loss between sensor and surface.

To orient inclinometer measurements in deep cased holes. 
TABLE 2.18

PROMISING IMPROVED INSTRUMENTS

\begin{tabular}{|c|c|}
\hline $\begin{array}{l}\text { PROMISING } \\
\text { IMPROVED } \\
\text { INSTRUMENTS }\end{array}$ & $\begin{array}{l}\text { APPLICABLE } \\
\text { IMPROVEMENTS } \\
\text { (parentheses indicate } \\
\text { improvement of lesser } \\
\text { importance) }\end{array}$ \\
\hline $\begin{array}{l}\text { SONDE TYPE BOREHOLE EXTENSOMETER } \\
\text { with } 2 \text { or } 3 \text { induction sensors } \\
\text { and casing collar markers }\end{array}$ & $\begin{array}{l}1,(3), 5,6,7,8,9,(10), 12, \\
15,16,(17), 18\end{array}$ \\
\hline $\begin{array}{l}\text { SONDE TYPE BOREHOLE EXTENSOMETER } \\
\text { with } 2 \text { or } 3 \text { gamma ray detectors } \\
\text { and radioactive bullet markers }\end{array}$ & $1,(3), 5,6,7,8,9,(10), 12$, \\
\hline $\begin{array}{l}\text { SONDE TYPE BOREHOLE EXTENSOMETER } \\
\text { with reed switch sensor and } \\
\text { magent markers }\end{array}$ & $\begin{array}{l}7,(3), 5,6,7,8,9,(10), 15, \\
16,17,18\end{array}$ \\
\hline $\begin{array}{l}\text { SONDE TYPE BOREHOLE INCLINOMETER } \\
\text { with servo-accelerometer }\end{array}$ & $\begin{array}{l}1,(3), 5,6,7,8,9,(10), 11, \\
12,15,16,18,19\end{array}$ \\
\hline $\begin{array}{l}\text { SONDE TYPE BOREHOLE INCLINOMETER } \\
\text { with cantilevered pendulum and } \\
\text { vibrating strain gauge sensors }\end{array}$ & $\begin{array}{l}1,(3), 5,6,7,8,9,(10), 11, \\
12,13,14,15,16,18,19\end{array}$ \\
\hline
\end{tabular}

${ }^{1}$ See Table 2.17 for 1 dentification of numbered improvements 
TABLE $\quad 2.19$

TRANSDUCER APPLICATION FOR GEOTHERMAL SUBSIDENCE

Sheet 1 of 2

\begin{tabular}{|c|c|c|}
\hline TRANSDUCER & RATING & COMENTS \\
\hline $\begin{array}{l}\text { Electrical Resistance } \\
\text { Strain Gauges } \\
\qquad\end{array}$ & $2-3$ & $\begin{array}{l}\text { Weldable strain gauges offer much promise because of good } \\
\text { stability at high temperatures. Bonded gauges are also } \\
\text { available for high temperature use, but are not as stable. } \\
\text { Both have successful field records. Downhole high } \\
\text { temperature signal conditioning wil be required to return } \\
\text { a good signal to the surface. Resistance and length } \\
\text { changes due to temperature will require compensation on } \\
\text { all resistance gauges. }\end{array}$ \\
\hline Potentiometers & 1 & $\begin{array}{l}\text { Suffering the same resistance problems as electrical } \\
\text { resistance strain gauges, potentiometers have additional } \\
\text { problems of friction, wiper contacts and current inavail- } \\
\text { ability for high temperature use. }\end{array}$ \\
\hline Capacitance Sensors & 1 & $\begin{array}{l}\text { Although they have good high temperature stability, they } \\
\text { are too fragile for field use. Also, moisture and signal } \\
\text { loss over long leads are problems. }\end{array}$ \\
\hline $\begin{array}{l}\text { Electromagnetic Vibrating } \\
\text { Wire Sensors }\end{array}$ & $2-3$ & $\begin{array}{l}\text { Their main asset is that the output signal is frequency } \\
\text { which is not sensitive to long. lead lengths. However, } \\
\text { the sensor is temperature sensitive and requires relative- } \\
\text { ly sophisticated signal conditioning including some } \\
\text { adjacent to the vibrating wire. It is not currently avail- } \\
\text { able in a high temperature package. Temperature compensa- } \\
\text { tion will be more complex than for resistance sensor. }\end{array}$ \\
\hline LVDTs and RVDTs & 2 & $\begin{array}{l}\text { LVTS are available in high temperature packages providing } \\
\text { the signal conditioning equipment is not at high tempera- } \\
\text { ture. LVDTs output a voltage signal and are thus sensitive } \\
\text { to long lead lengths. High temperature signal conditioning } \\
\text { for downhole use will be required to return a good signal. }\end{array}$ \\
\hline $\begin{array}{l}\text { Electrical Force Balance } \\
\text { Transducers }\end{array}$ & 2 & $\begin{array}{l}\text { Because they minimize vibrations, these have been the most } \\
\text { accurate sensors for tilt measurements. However, they are } \\
\text { not available in high temperature packages. Both the } \\
\text { restoring coil system and the proximity sensor would } \\
\text { require redesign to compensate for length, electrical } \\
\text { resistance and magnetic permeability changes due to tem- } \\
\text { perature. }\end{array}$ \\
\hline $\begin{array}{l}\text { Magnetic Induction } \\
\text { Sensors }\end{array}$ & 2 & $\begin{array}{l}\text { Their main asset is that they have been used in downhole } \\
\text { probes to make accurate measurements of settlement. They } \\
\text { are not currently available in high temperature packages. } \\
\text { Redesign would have to compensate for lead length, } \\
\text { electrical resistance and magnetic permeability changes } \\
\text { due to temperature. }\end{array}$ \\
\hline $\begin{array}{l}\text { Time Doma in } \\
\text { Reflectometers }\end{array}$ & 1 & $\begin{array}{l}\text { Although advantageous because no downhole electronics or } \\
\text { probes are required, this method is too inaccurate. Also } \\
\text { with numerous markers, it is hard to analyze. }\end{array}$ \\
\hline Reed Switch Sensors & 3 & $\begin{array}{l}\text { Very promising because only simple downhole electronics } \\
\text { would be required. The signal is off-on, another good } \\
\text { feature. The effects of temperature on the strength of } \\
\text { the downhole magnets and on the permeability of the reed } \\
\text { switch blades must be assessed. Corrosion of the magnets } \\
\text { may be a problem. }\end{array}$ \\
\hline $\begin{array}{l}\text { Optical Fiber } \\
\text { Transducers }\end{array}$ & 1 & $\begin{array}{l}\text { Problems arise because of fragility of fibers and depen- } \\
\text { dency on high-powered lasers. However, it may offer } \\
\text { promise at a later date due to resistance to hostile } \\
\text { environments and stray electromagnetic fields. }\end{array}$ \\
\hline
\end{tabular}

DEFINITION OF

RATING RATING CATEGORY

2 Some Promise

3 Very Promising 
TABLE 2.19

TRANSDUCER APPLICATION FOR GEOTHERMAL SUBSIDENCE (CONT'D)

Sheet 2 of 2

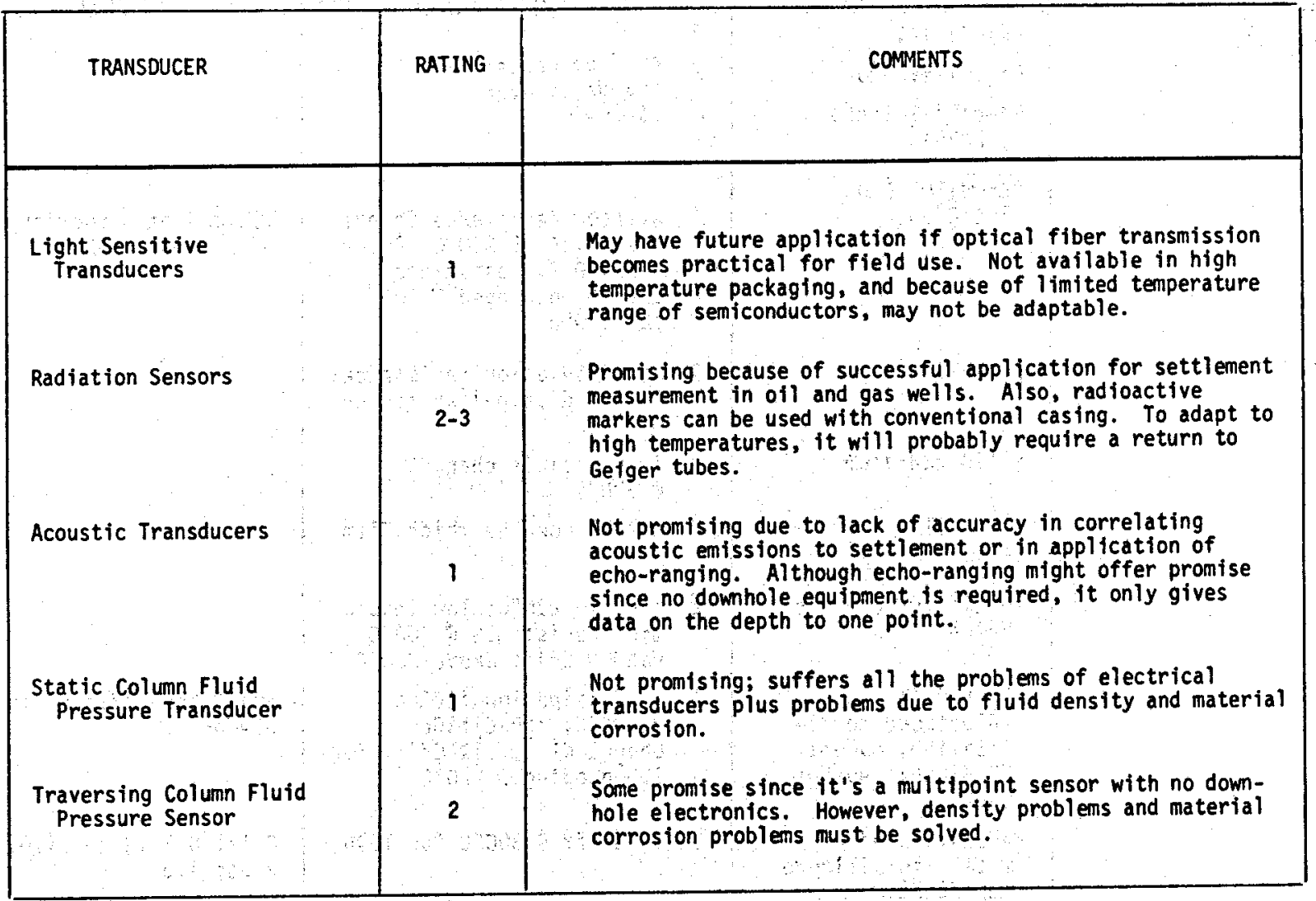


TABLE 2.20

PERFORMANCE OF CIRCUIT ELEMENTS AT $300^{\circ} \mathrm{C}$

Sheet 1 of 2

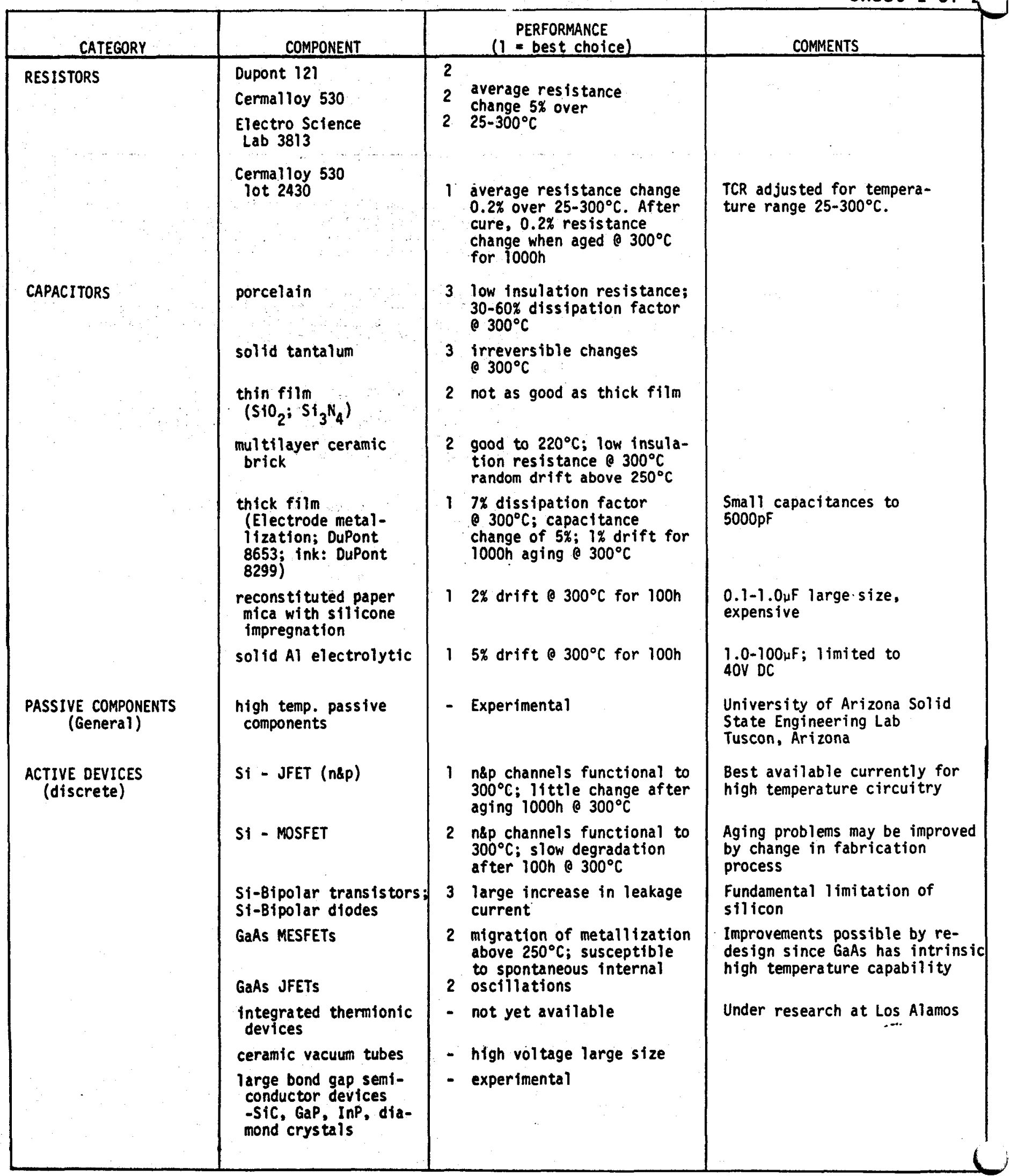


TABLE 2.20

PERFORMANCE OF CIRCUIT ELEMENTS AT $300^{\circ} \mathrm{C}$ (CONT'D)

Sheet 2 of 2

\begin{tabular}{|c|c|c|c|}
\hline CATEGORY & COMPONENT & $\begin{array}{c}\text { PERFORMANCE } \\
(1=\text { best choice })\end{array}$ & COMMENTS \\
\hline $\begin{array}{l}\text { ACTIVE DEVICES } \\
\text { (integrated circuits) } \\
\text { COMPLETE CIRCUITRY } \\
\text { BONDING }\end{array}$ & $\begin{array}{l}\text { integrated circuits } \\
\text { ultrahigh temperature } \\
\text { amplifier } \\
\text { Au-Au } \\
\text { Au-Al } \\
\text { Al wire bonded direct- } \\
\text { ly to Cermalloy } 4399 \\
\text { or DuPont } 9791 \text { Au thick } \\
\text { film conductor } \\
\text { Al-wire bonded directly } \\
\text { to tolerant Pt-Au thick } \\
\text { film conducted; Al bond- } \\
\text { ed ultrasonically } \\
\text { Al-wire bonded directly } \\
\text { to modified Au Dupont } \\
9910 \text { thick film } \\
\text { materials } \\
\text { Buffer: Ni or silicon } \\
\text { discs between Au thick } \\
\text { film and Al wire } \\
\text { Au ribbon; parallel gap } \\
\text { welded to Pt-Au con- } \\
\text { ducting layer } \\
\text { Au/Ge solder }\end{array}$ & 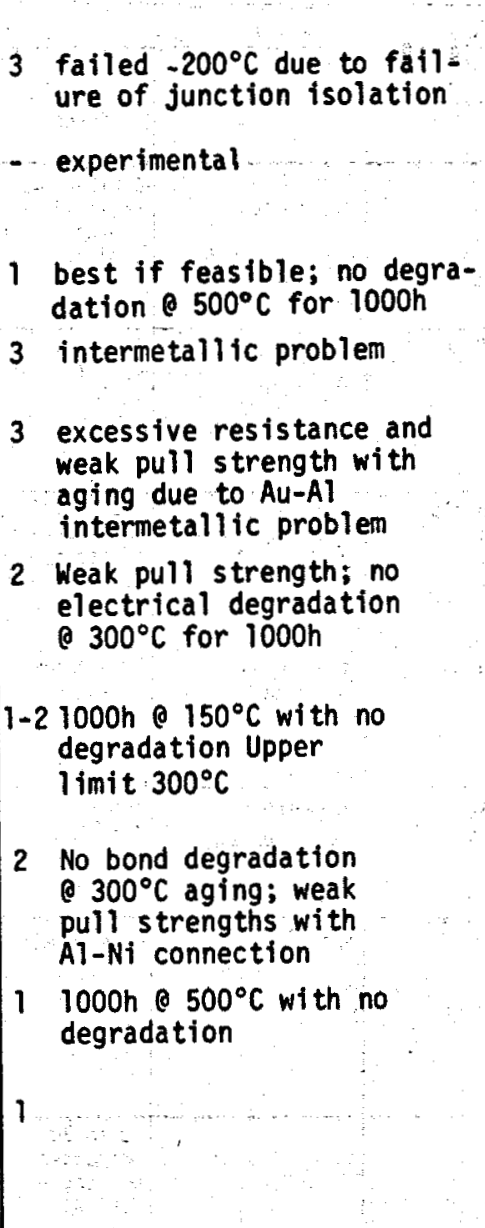 & $\begin{array}{l}\text { Improvements possible with } \\
\text { dielectric isolation } \\
\text { System Development Corp., } \\
\text { Santa Monica, California } \\
\text { Requires semi-conductor device } \\
\text { with Au metallization } \\
\text { Aging degradation produces high } \\
\text { reststance connection } \\
\qquad \\
\text { attach active devices in To } \\
\text { cans to printed circuit boards } \\
\text { and smali discrete components } \\
\text { to hybrid substrates } \\
\text { for Al wire systems } \\
\text { Add-on component bonding } \\
\text { and crossovers } \\
\text { Ans }\end{array}$ \\
\hline
\end{tabular}

(Palmer et al, 1978; McBrayer et al, 1977; Palmer, 1977; Baker, Campbell and Hughen, 1975) 


\section{T A B L E 2.21}

SELECTED RECORDER SYSTEMS

(For Above Ground Data Recording)

\begin{tabular}{|c|c|c|c|c|c|c|c|}
\hline $\begin{array}{l}\text { TYPE OF } \\
\text { RECOROER }\end{array}$ & APPLICATION & $\begin{array}{l}\text { MAXINEM } \\
\text { NO. OF } \\
\text { CHANNELS }\end{array}$ & $\begin{array}{l}\text { RANGE } \\
\text { CHART } \\
\text { SPEED }\end{array}$ & $\frac{\text { RECORDIN }}{\underbrace{\text { SPEED }}_{\text {WRITING }}}$ & $\begin{array}{l}\text { G RA TES } \\
\text { RATES FOR } \\
\text { EVENTS/POINTS }\end{array}$ & ACCURACY & $\begin{array}{l}\text { ADVANTAGES \& } \\
\text { DISADVANTAGES }\end{array}$ \\
\hline EVENT & $\begin{array}{l}\text { Yes-No or } \\
\text { On-Off events; } \\
\text { suitable for use } \\
\text { with reed switch. } \\
\text { contact closure } \\
\text { devices. }\end{array}$ & $\begin{array}{l}\text { Up to } 40 \\
\text { channels } \\
\text { typical; } \\
\text { Some to } 150 \\
\text { channels }\end{array}$ & $\begin{array}{l}6 \mathrm{~nm} / \mathrm{hr}- \\
200 \mathrm{~mm} / \mathrm{sec} \\
\text { Use } 30 \text { strokes } \\
\text { per inch of } \\
\text { chart }\end{array}$ & $\begin{array}{l}0.015-0.02 \\
\text { sec-fuli scale } \\
\text { typtcal; } 0.0010 \\
\text { available }\end{array}$ & $\begin{array}{l}\text { up to } 20 \\
\text { events } / \text { sec } \\
\max \end{array}$ & $\begin{array}{l}\text { system } \\
\text { dependence } \\
\text { approx } 0.25 \% \\
\text { to } 2 \%\end{array}$ & $\begin{array}{l}\text { Inexpensive; reliable. } \\
\text { Tedious to analvze the data. }\end{array}$ \\
\hline MULTIPOINT & $\begin{array}{l}\text { Generally used } \\
\text { for slowly- } \\
\text { changing indus- } \\
\text { trial operations; } \\
\text { many variables } \\
\text { vs. time }\end{array}$ & $\begin{array}{l}\text { Up to } 48 \\
\text { Channels }\end{array}$ & $\begin{array}{l}6 \mathrm{~mm} / \mathrm{hr}- \\
100 \mathrm{~mm} / \mathrm{min}\end{array}$ & $\begin{array}{l}0.3 \text { to } 7.0 \\
\text { sec fuli } \\
\text { scale } \\
\text { response }\end{array}$ & $\begin{array}{l}2 \text { secs/point } \\
\text { to } 16 \text { points/ } \\
\text { sec }\end{array}$ & $\begin{array}{l}0.25 \%+\text { of } \\
\text { full scale }\end{array}$ & $\begin{array}{l}\text { Very slow; not computer } \\
\text { compatible. }\end{array}$ \\
\hline $\begin{array}{l}\text { STRIP } \\
\text { CHART }\end{array}$ & $\begin{array}{l}\text { Varlable input- } \\
\text { such as current, } \\
\text { yoltage of strain } \\
\text { transducers vs. } \\
\text { time }\end{array}$ & $\begin{array}{l}1-4 \text { channels } \\
\text { typical: } \\
\text { Up to } 30 \\
\text { possible }\end{array}$ & $\begin{array}{l}4 \mathrm{~mm} / \mathrm{hr} \\
200 \mathrm{~mm} / \mathrm{sec} \\
\text { typical: }\end{array}$ & $\begin{array}{l}0.3 \text { to } 1.0 \text { sec- } \\
\text { full scale re- } \\
\text { sponse typical: } \\
0.12 \text { sec avail. }\end{array}$ & $\begin{array}{l}2-4 \mathrm{~Hz} \\
\text { typical: } \\
\text { Available } \\
\text { to } 125 \mathrm{~Hz}\end{array}$ & $\begin{array}{l}0.25 \% \text { to } \\
0.5 \% \text { full } \\
\text { scale } \\
\text { typical; } \\
1-2 \% \text { at high } \\
\text { frequency }\end{array}$ & $\begin{array}{l}\text { Reliable; cheap compared to } \\
\text { magnetic tape. Not computer } \\
\text { compatible; avaflable with } \\
\text { galvanometric or servo- } \\
\text { potentioneter mechanisms; } \\
\text { tedious to analyze }\end{array}$ \\
\hline $\begin{array}{l}\text { CIRCULAR } \\
\text { OR } \\
\text { ROTARY } \\
\text { CHART }\end{array}$ & $\begin{array}{l}\text { As above; usually } \\
\text { dedicated to } \\
\text { single variable } \\
\text { such as tempera- } \\
\text { ture, water level }\end{array}$ & $1-2$ tyoical & $\begin{array}{l}4 \mathrm{hr}-8 \text { day } \\
\text { rotation }\end{array}$ & $\begin{array}{c}\text { Very slow } \\
\vdots \\
\vdots\end{array}$ & per minute & $\begin{array}{l}\text { relative } \\
\text { to } 24 \text { hour } \\
\text { system }\end{array}$ & $\begin{array}{l}\text { Too slow for most appli- } \\
\text { cations; data not compu- } \\
\text { ter compatible. }\end{array}$ \\
\hline $\begin{array}{l}\text { OSCILLOGRAPH } \\
\text { RECORDER }\end{array}$ & $\begin{array}{l}\text { Rapid events, } \\
\text { such as } \\
\text { geophysical p } \\
\text { and s-wave } \\
\text { tests }\end{array}$ & $\begin{array}{l}\text { Up to } 16 \\
\text { Channels }\end{array}$ & $\begin{array}{l}0.5 \mathrm{~mm} / \mathrm{sec} \\
\text { to } 1.3 \mathrm{~m} / \mathrm{sec}\end{array}$ & up to $350 \mathrm{~m} / \mathrm{sec}$ & $\begin{array}{l}10-1000 \mathrm{~Hz} \\
\text { full scale; }\end{array}$ & $\begin{array}{l}5 \% \text { full } \\
\text { scale }\end{array}$ & $\begin{array}{l}\text { Not suitable for field use - } \\
\text { delicate, not easily portable }\end{array}$ \\
\hline $\begin{array}{l}\text { STORAGE } \\
\text { OSCILLOSCOPE }\end{array}$ & $\begin{array}{l}\text { Store events up } \\
\text { to } 450 \mathrm{KHZ} \text { : } \\
\text { Store size: } \\
\text { 1024 } \times 8 \text { bits } \\
\text { Permanent record } \\
\text { can be made on } \\
\text { strip chart } \\
\text { recorder }\end{array}$ & $1-2$ & $N / A$ & Very fast & Very fast & $\begin{array}{l}+3 \% \\
\text { Typical }\end{array}$ & $\begin{array}{l}\text { Data difficult to reduce. } \\
\text { Difficult to interface to a } \\
\text { computer. Excellent for } \\
\text { rapid data examination. }\end{array}$ \\
\hline $\begin{array}{l}\text { PAPER } \\
\text { TAPE } \\
\text { PERFORATOR }\end{array}$ & $\begin{array}{l}\text { Digital data } \\
\text { recording }\end{array}$ & $\begin{array}{c}1-2 \\
\text { Typical }\end{array}$ & $\rightarrow$ & $\begin{array}{l}10 \text { to } 30 \\
\text { characters } \\
\text { per second } \\
\text { Typical }\end{array}$ & $\begin{array}{l}\text { Encoding } \\
\text { dependent }\end{array}$ & $\begin{array}{l}\text { digital } \\
\text { dropout } \\
\text { rate may be } \\
\text { significant }\end{array}$ & $\begin{array}{l}\text { Cheaper than mag. tape at } \\
\text { low speed; as expensive at } \\
\text { high speed; moderate } y \text { ac- } \\
\text { curate; can feed into al- } \\
\text { most any teletype; inter- } \\
\text { faces to most computers; } \\
\text { not sensitive to dust \& } \\
\text { field conditions; possi- } \\
\text { ble to verify operation by } \\
\text { looking for certain punch } \\
\text { patterns. }\end{array}$ \\
\hline $\begin{array}{l}\text { MAGNETIC } \\
\text { TAPE }\end{array}$ & $\begin{array}{l}\text { For large quanti- } \\
\text { ties of data; } \\
\text { Also for future } \\
\text { analys is of data } \\
\text { by computer }\end{array}$ & $\begin{array}{l}6 \text { channels } \\
\text { with data } \\
\text { acquisition } \\
\text { system; } 7 \text { or } \\
9 \text { track tape } \\
\text { typical }\end{array}$ & $\begin{array}{l}23 \mathrm{~mm} / \mathrm{sec}- \\
6 \mathrm{~m} / \mathrm{sec} \\
\text { Each track } \\
\text { req's sig- } \\
\text { nal cond. }\end{array}$ & $\begin{array}{l}\text { Very fast: } \\
\text { compatible } \\
\text { with computers }\end{array}$ & & $\begin{array}{l}\text { Excellent; } \\
\text { dropout rate } \\
\text { for digital } \\
\text { data is } \\
\text { negligible }\end{array}$ & $\begin{array}{l}\text { Band width } 2 \mathrm{OHz} \text { to } 2 \mathrm{MHz} \text {; } \\
\text { faster, more info than paper } \\
\text { tape; cheaper models may not } \\
\text { interface with many computer } \\
\text { installations. Good way } \\
\text { to store data a long time; } \\
\text { Relatively expensive; sensi- } \\
\text { tive to dirt. }\end{array}$ \\
\hline $\begin{array}{l}\text { PHOTO- } \\
\text { GRAPHIC }\end{array}$ & $\begin{array}{l}\text { Use with CRT, } \\
\text { downhole instru- } \\
\text { ments requiring } \\
\text { 2-D records, } \\
\text { such as compasses } \\
\text { and pendulums }\end{array}$ & $\stackrel{1}{\text { Typical }}$ & $\begin{array}{l}\text { Relatively } \\
\text { slow } \\
\text { (typically) }\end{array}$ & & & $\begin{array}{l}\text { Can be very } \\
\text { useful for } \\
\text { extensive } \\
\text { data } \\
\text { compression }\end{array}$ & $\begin{array}{l}\text { Special purpose - data } \\
\text { difficuit to process. }\end{array}$ \\
\hline
\end{tabular}




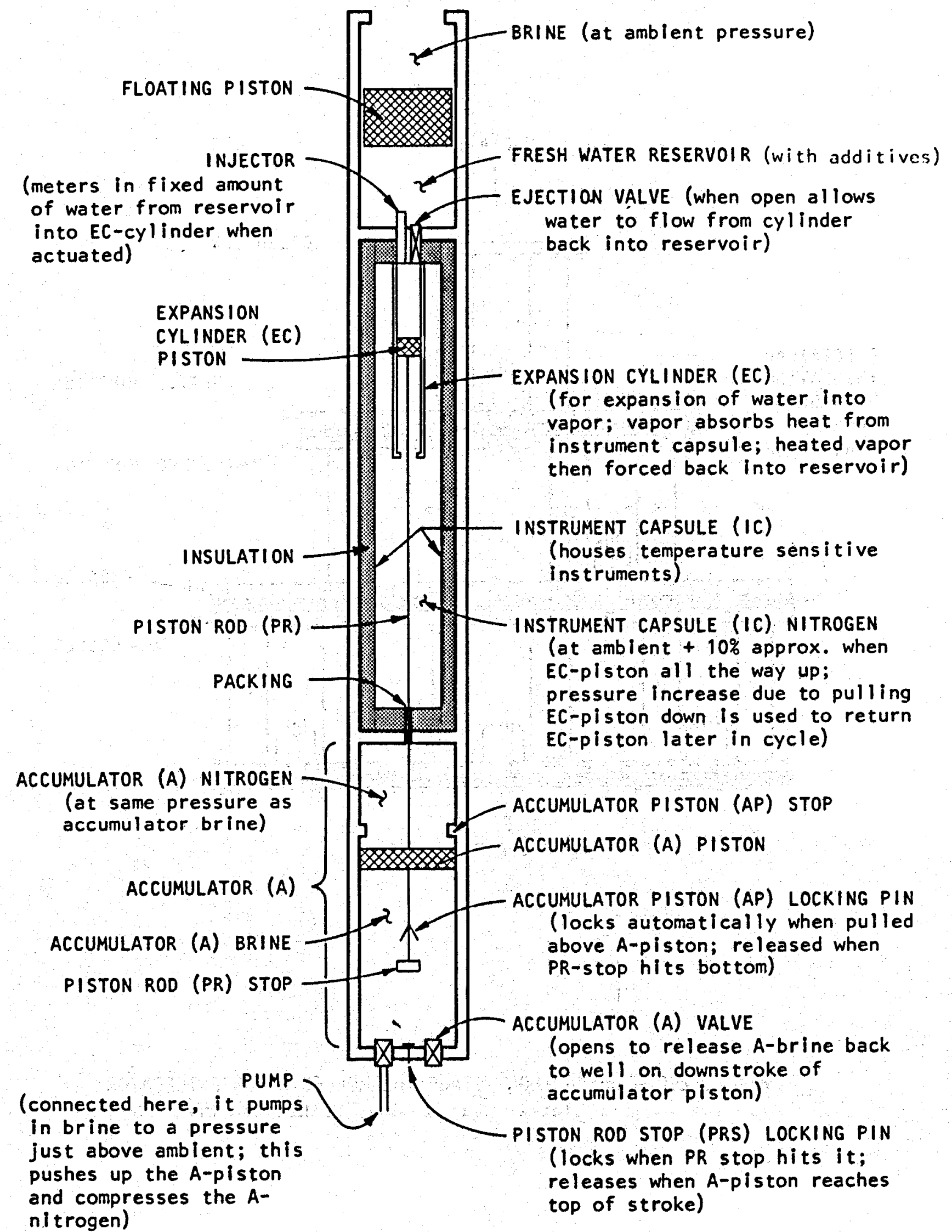

(meters in fixed amount of water from reservoir into EC-cylinder when actuated)

Figure 2.1 - BASIC ELEMENTS OF POSSIBLE MECHANICAL HEAT PUMP 


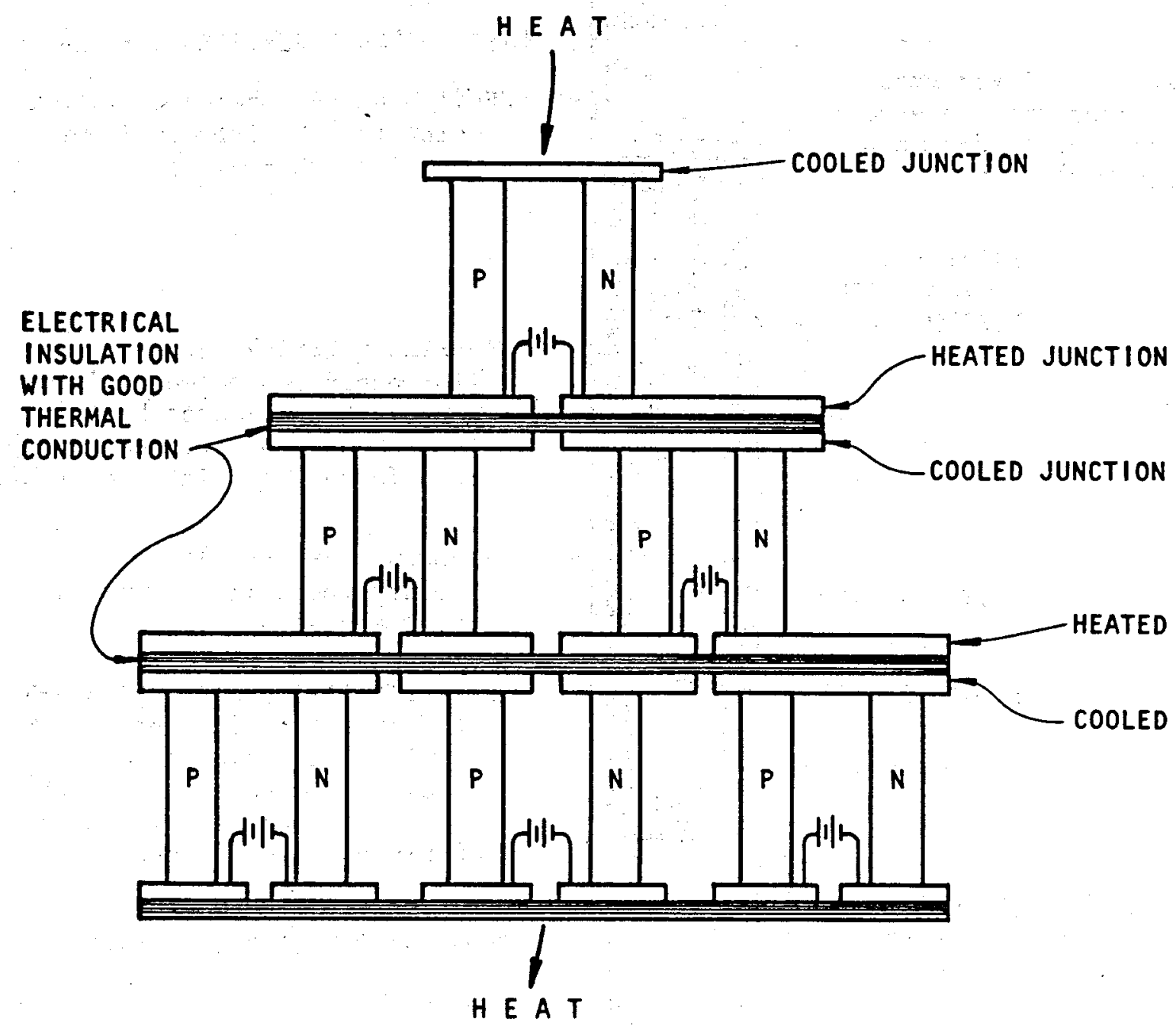

Figure 2.2 - CASCADING 3-STAGE THERMOELECTRIC REFRIGERATOR

(after Wolfe, 1962; Heikes and Ure, 1960) 


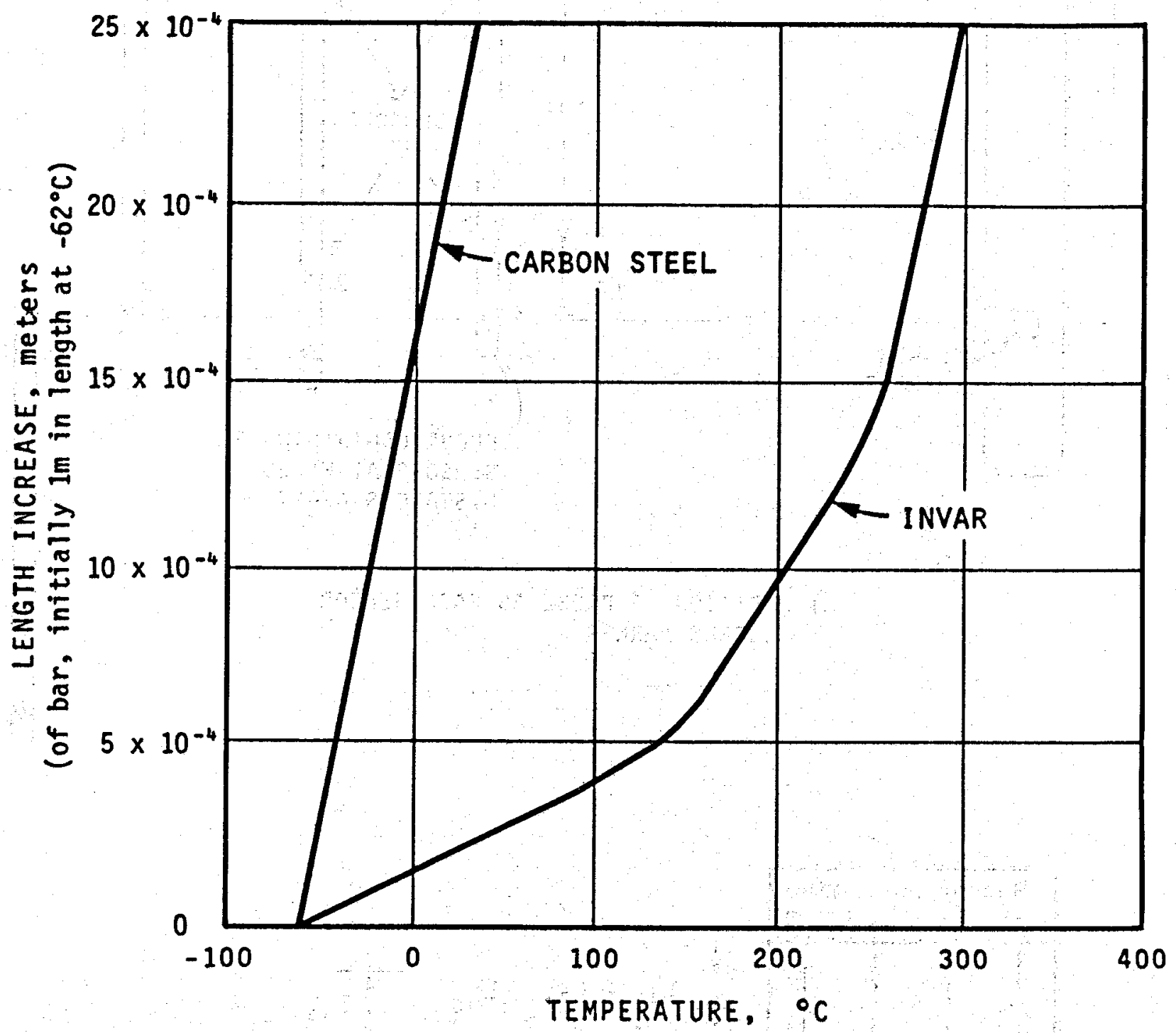

Figure 2.3 - RELATION BETWEEN LENGTH INCREASE AND TEMPERATURE FOR CARBON STEEL AND INVAR

(after Carpenter steel Division, 1976) 

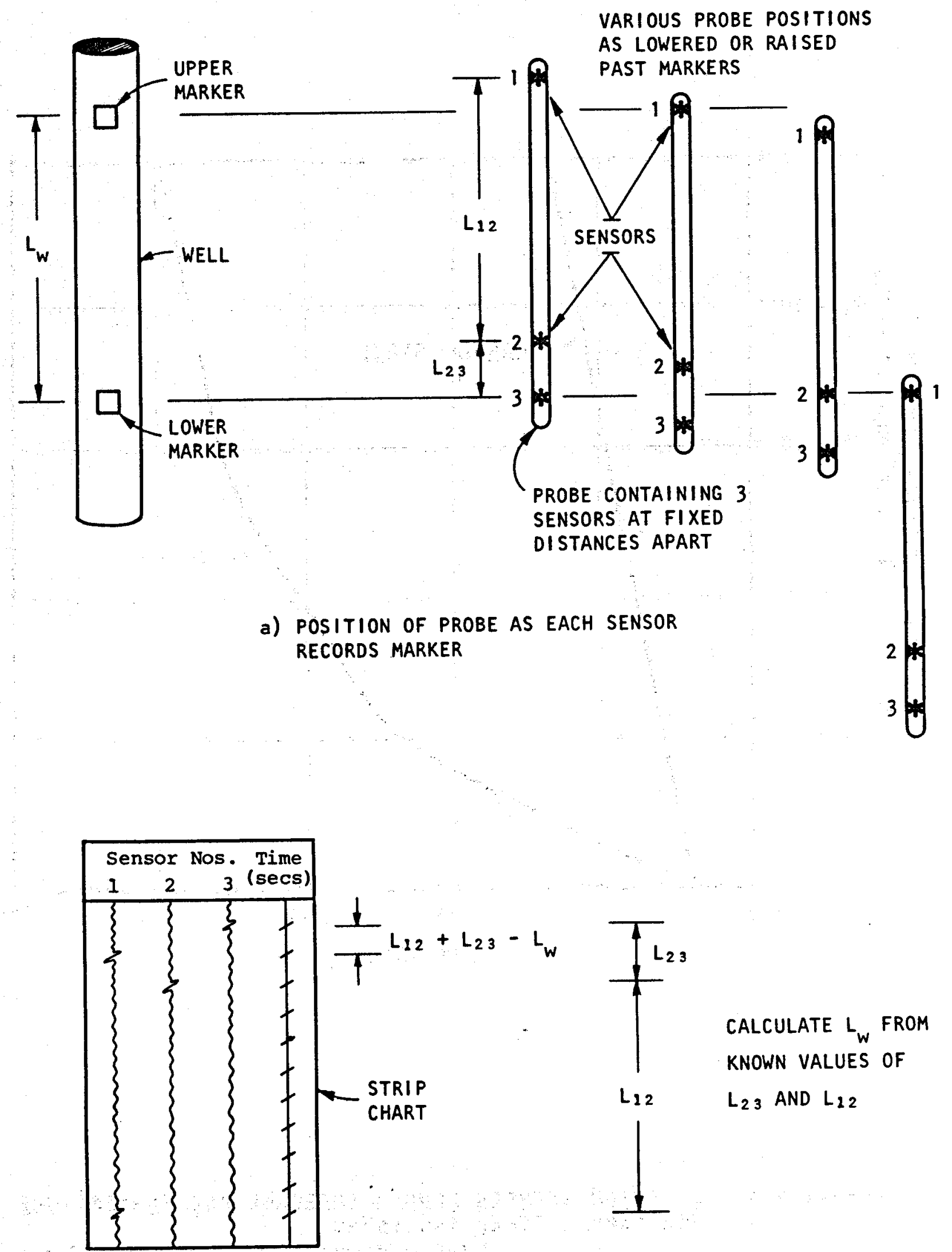

b) DETERMINATION OF DISTANCE BETWEEN MARKERS FROM STRIP CHART

Figure 2.4 - USE OF THREE SENSOR PROBE TO DETERMINE DISTANCE BETWEEN DOWNHOLE MARKERS 

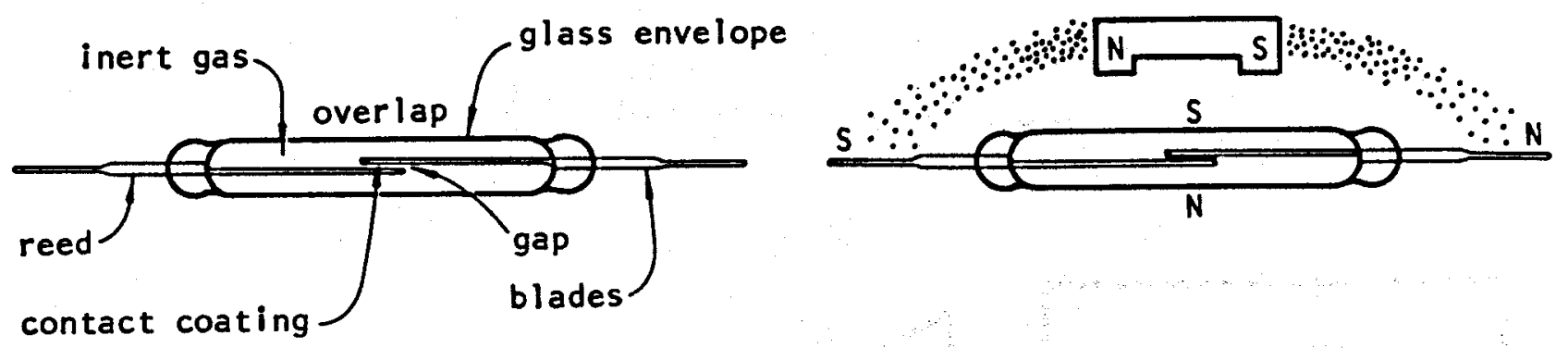

a) REED SWITCH COMPONENTS
(F-R Electronics, 1972)

b) NORMAL OPERATING POSITION OF SWITCH

(F-R Electronics, 1972)

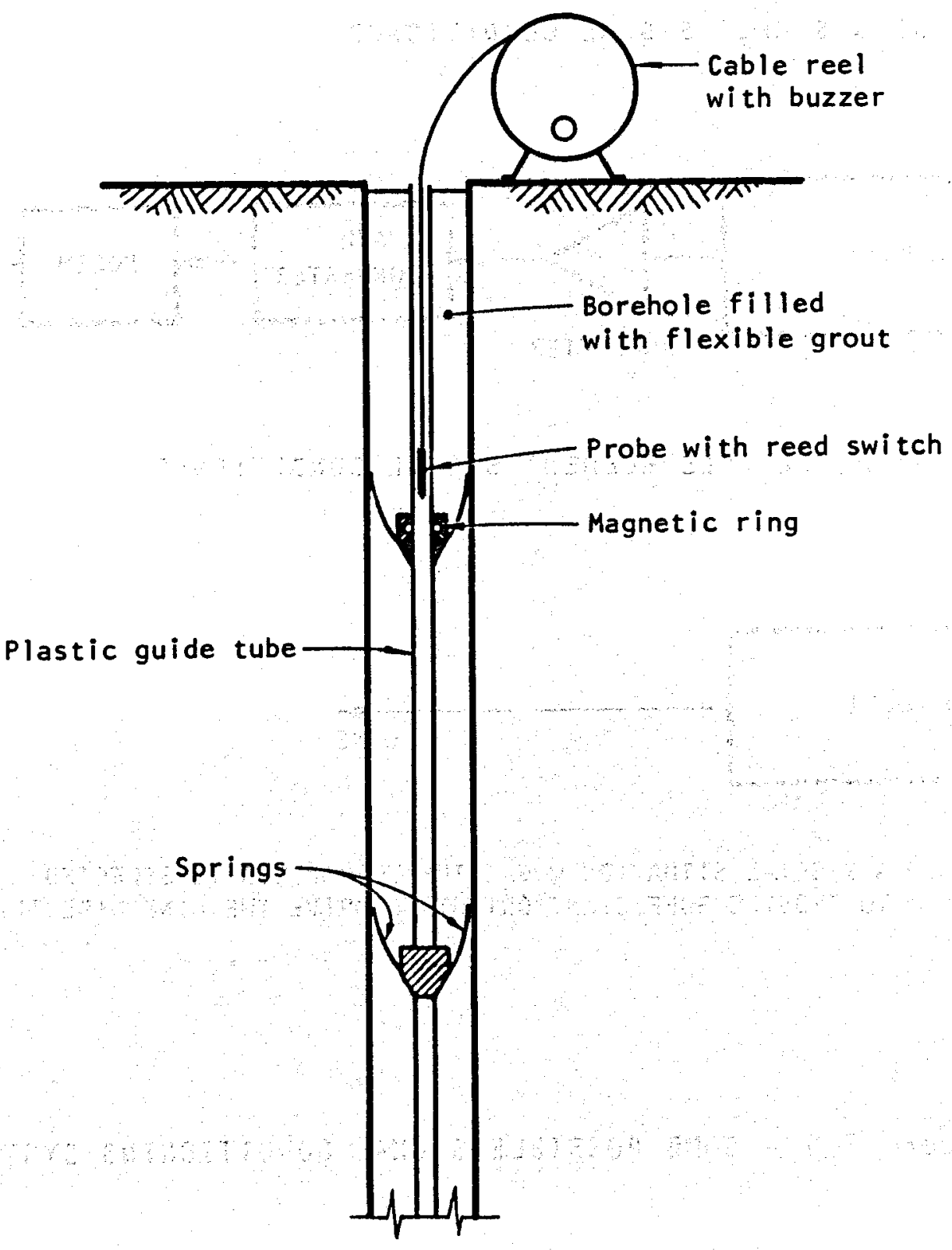

c) APPLICATION OF REED SWITCH IN GEOTECHNICAL ENGINEERING

Figure 2.5 - REED SWITCH SENSOR 


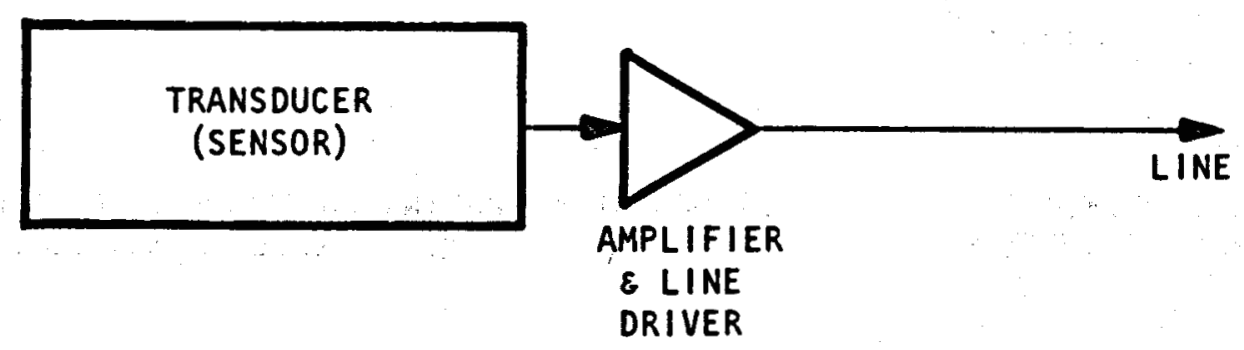

a) A SIMPLE SIGNAL CONDITIONER

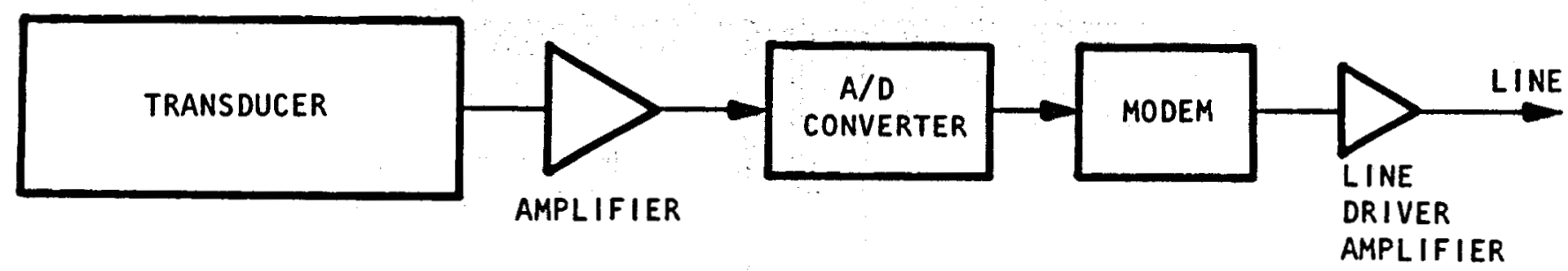

b) A MULTIPLE ELEMENT SIGNAL CONDITIONER

TRANSDUCER

c) A SPECIAL SITUATION WHERE THE TRANSDUCER IS SELECTED TO PROVIDE SUFFICIENT OUTPUT TO DRIVE THE LINE DIRECTLY

Figure 2.6 - SOME POSSIBLE SIGNAL CONDITIONING SYSTEMS 


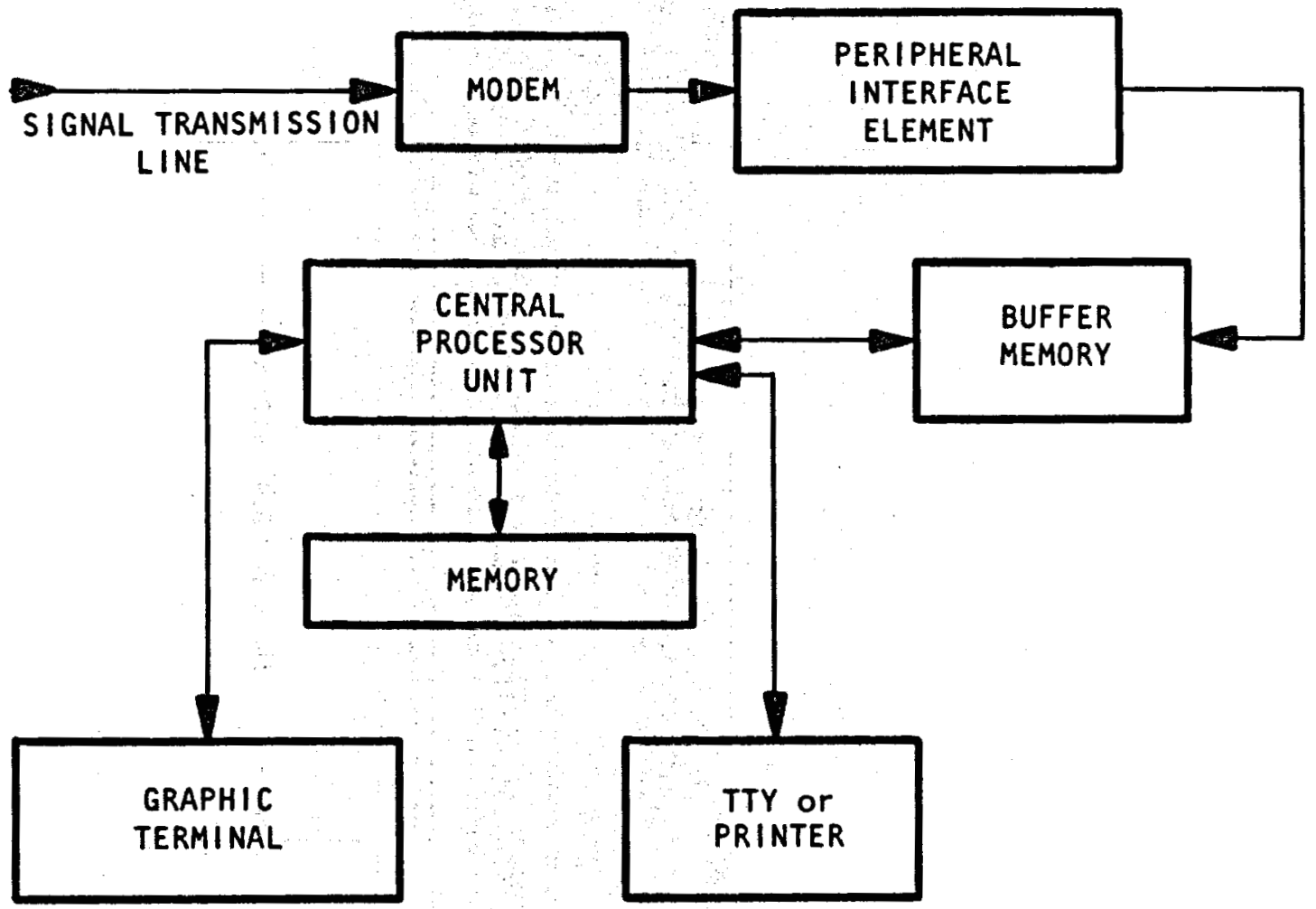

Figure 2.7 - A POSSIBLE ABOVE-GROUND COMPUTER STATION (Either a mini-computer or micro-computer system is applicable) 


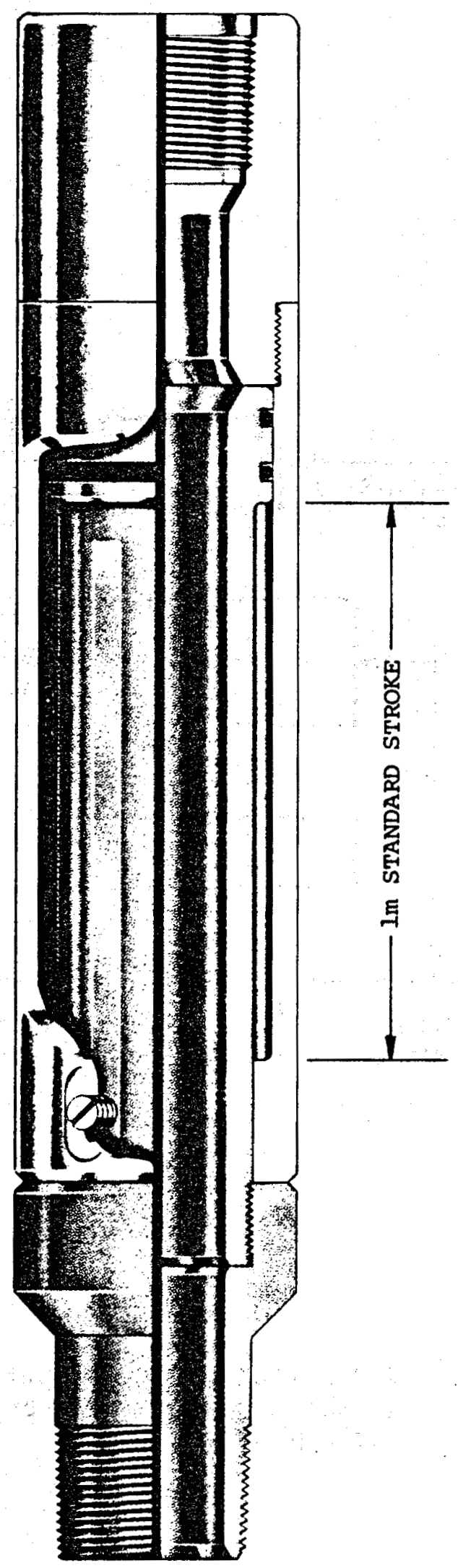

a) COMMERCIAL CASING EXPANSION JOINT

(after Lynes, 1978)

Figure 2.8 - SLIP COUPLINGS FOR SUBSIDENCE MONITORING 


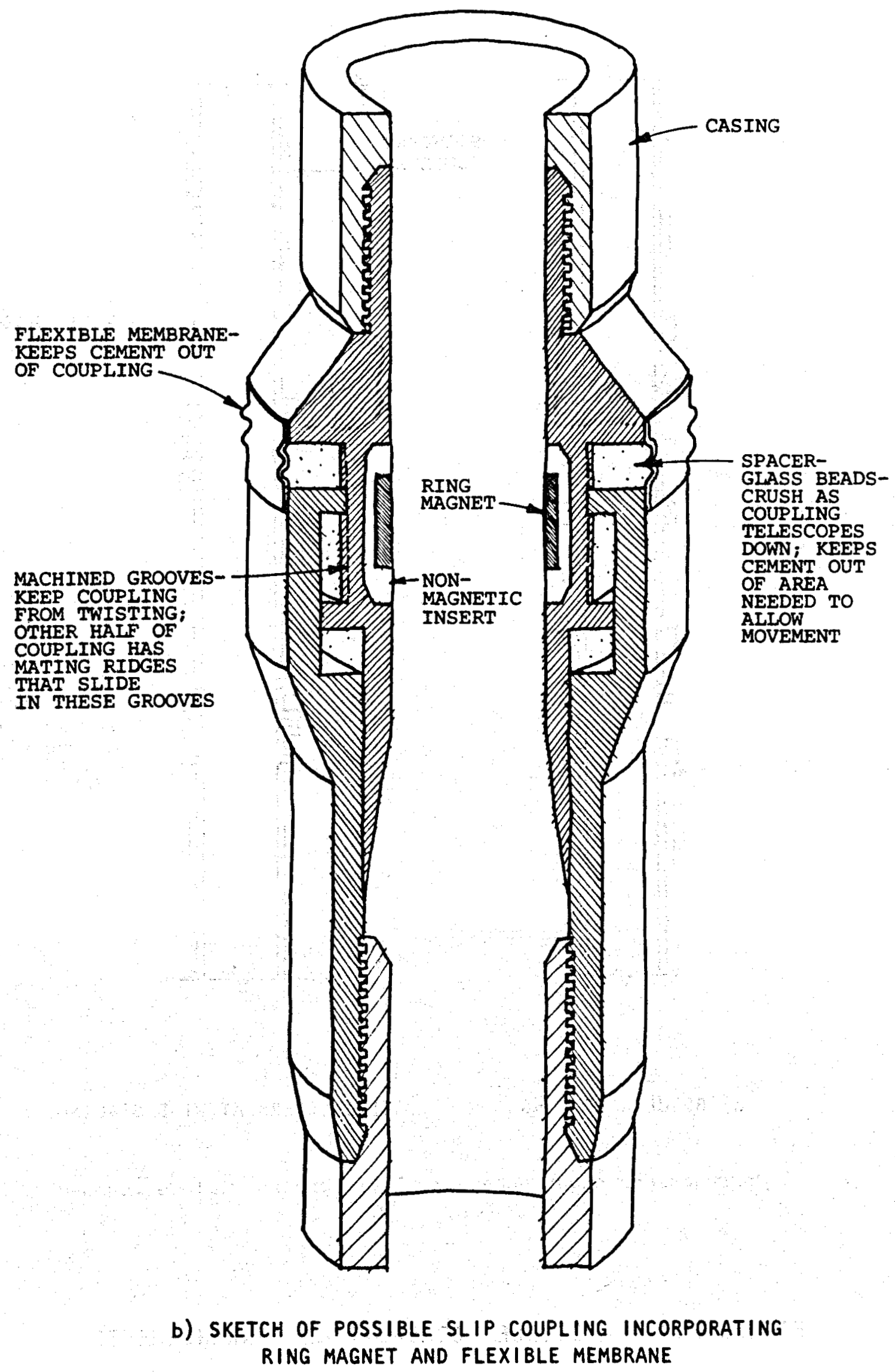

Figure 2.8 - SLIP COUPLINGS FOR SUBSIDENCE MONITORING (CONT'D) 


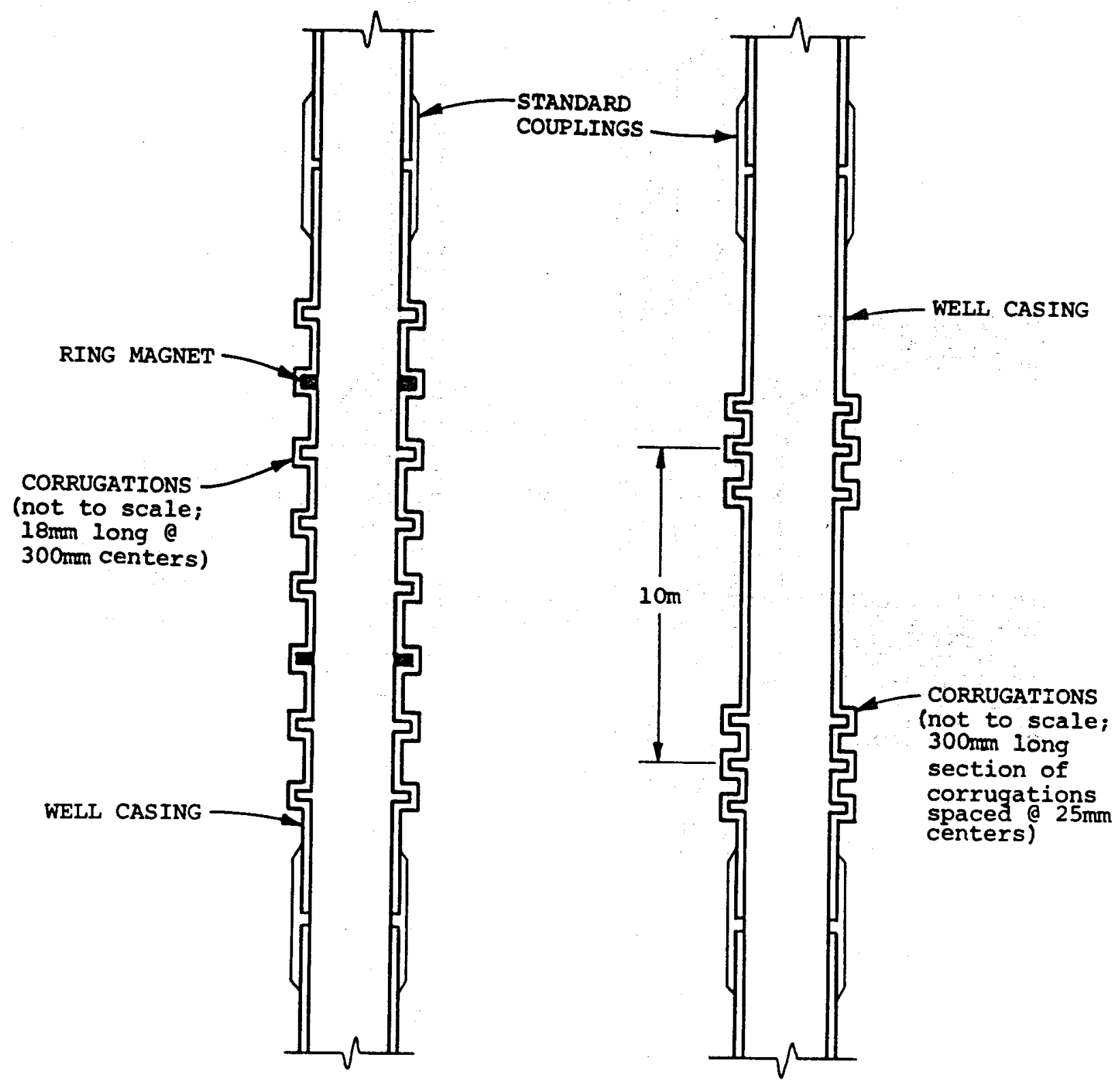
a) REGULAR SPACING
b) CLUSTERS AT WIDE SPACING

Note: Corrugations shown schematically; corners will be rounded

Figure 2.9 - POSSIBLE CORRUGATION ARRANGEMENTS 


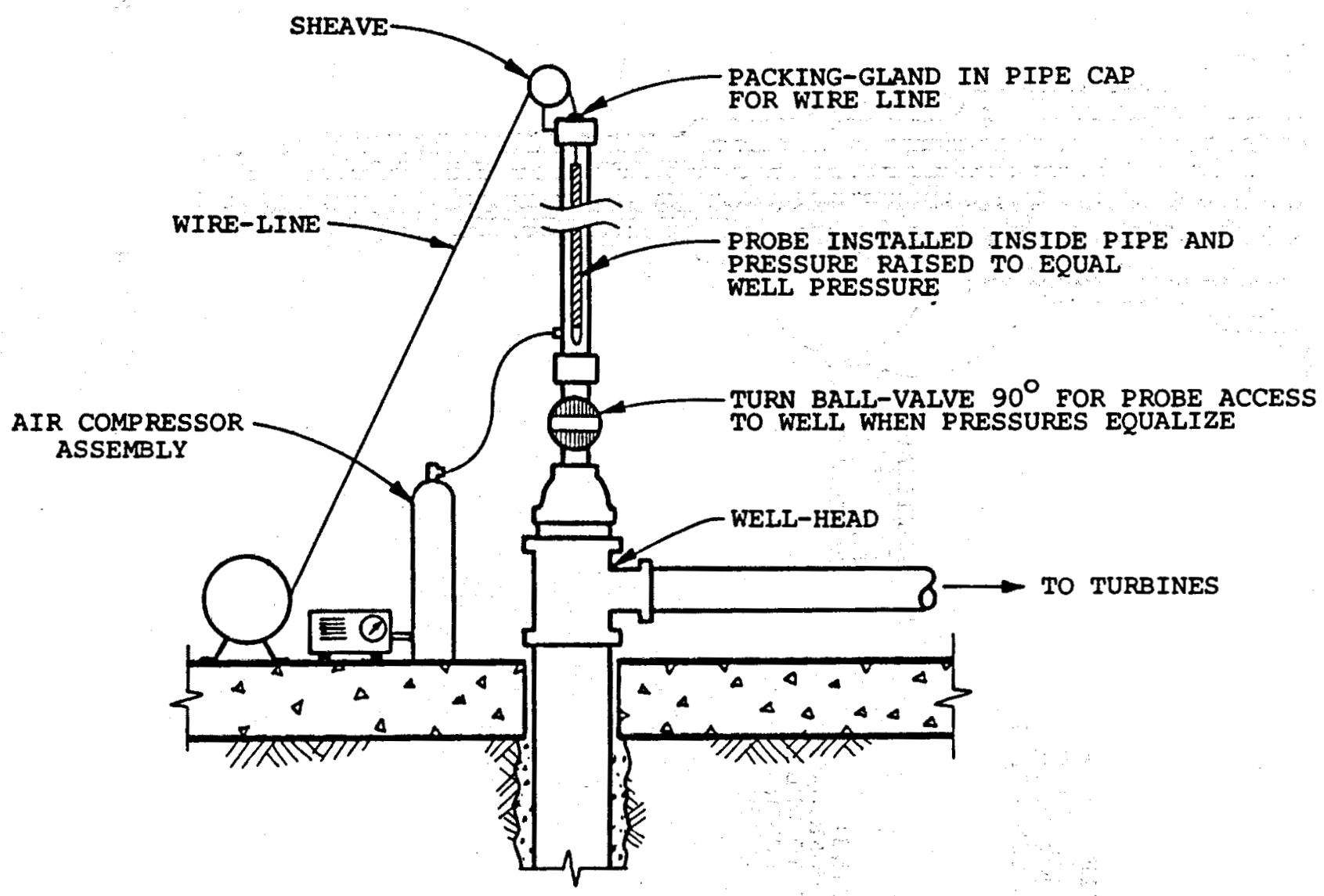

NOTE : ALL EQUIPMENT EXCEPT PROBE IS LEFT AT WELL HEAD.

Figure 2.10 - ACCESS GLAND FOR SETTLEMENT PROBE 


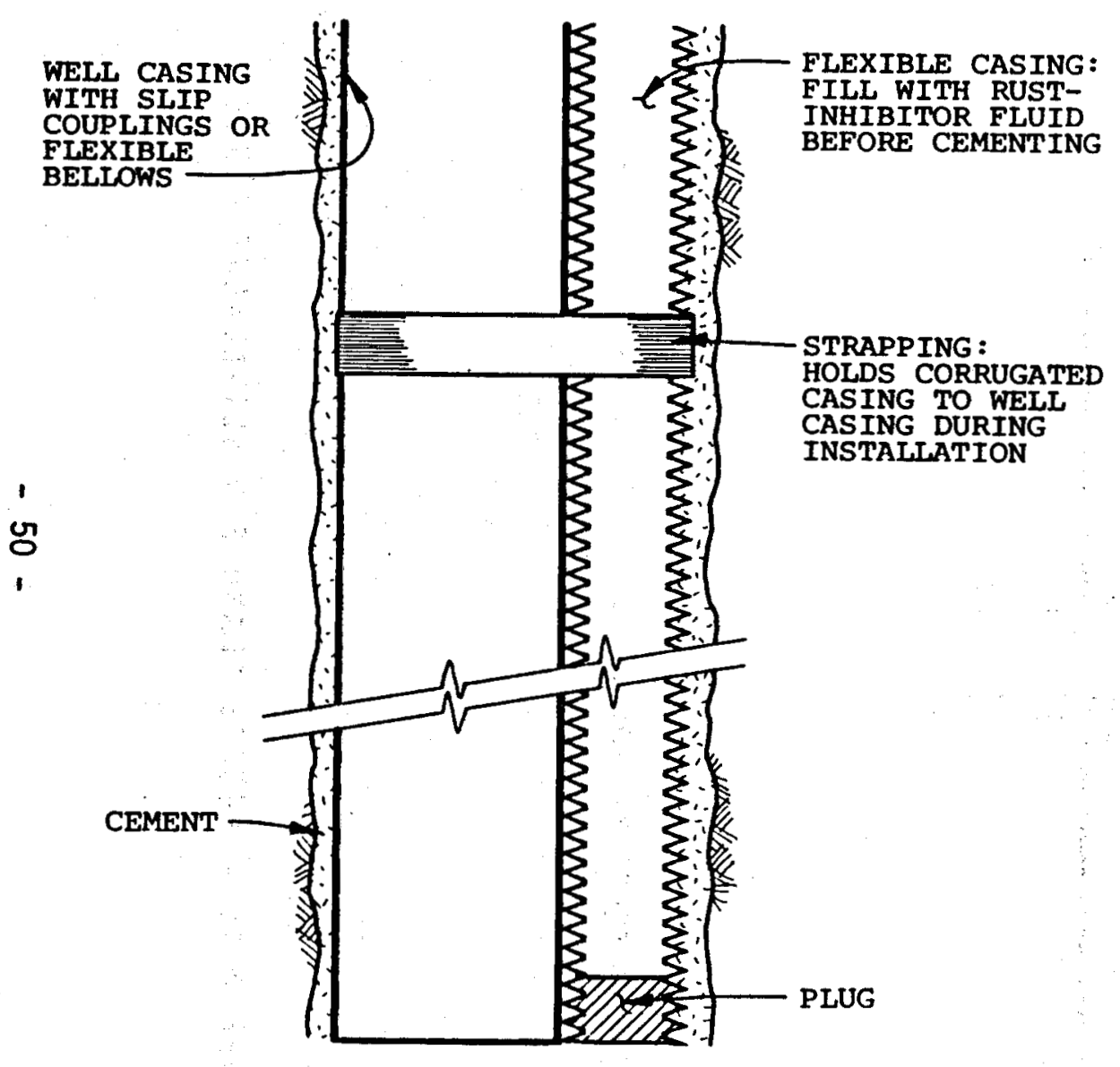

a) SCHEMATIC OF INSTALLATION

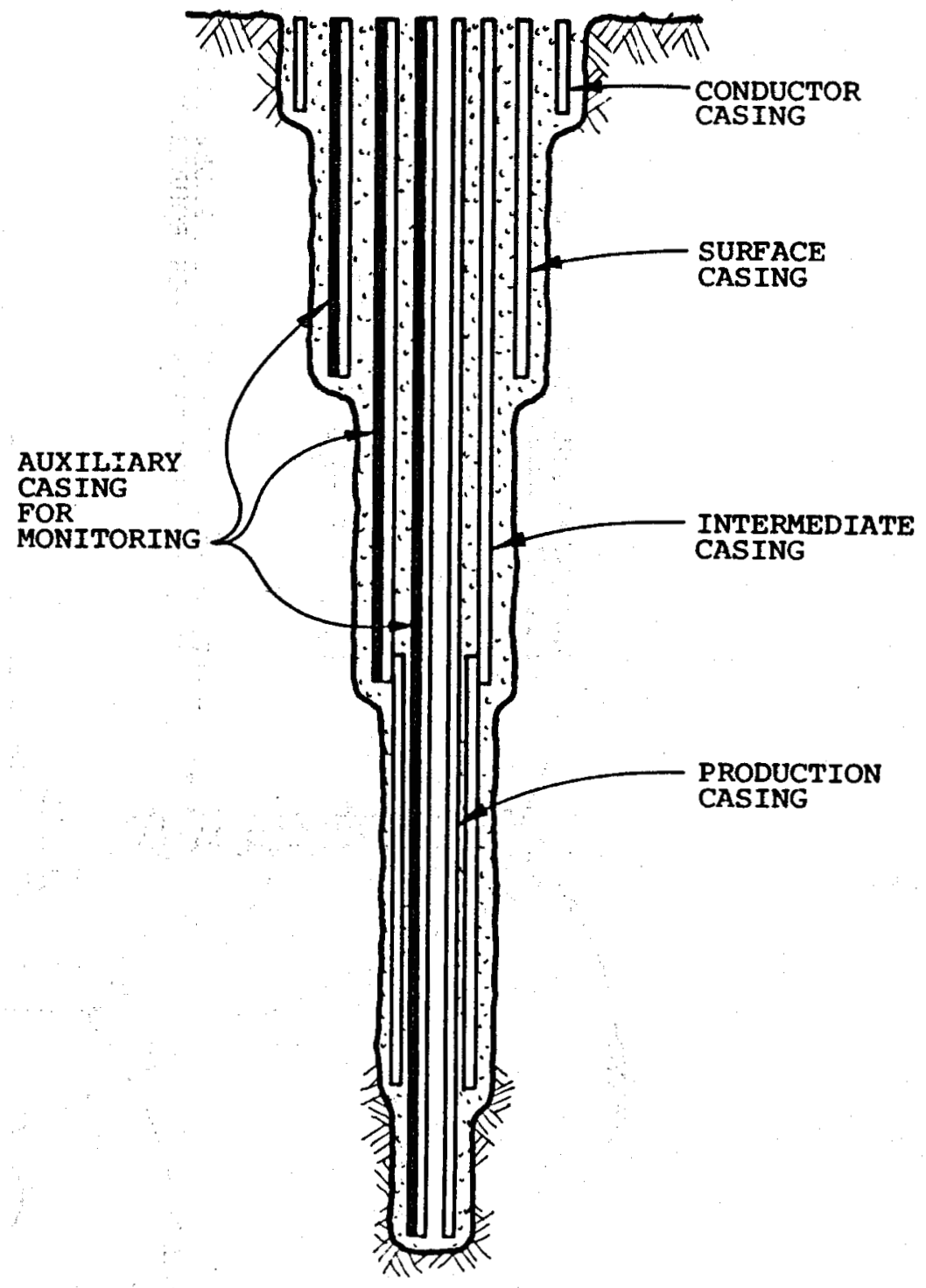

b) USE OF MULTIPLE AUXILIARY CASING 


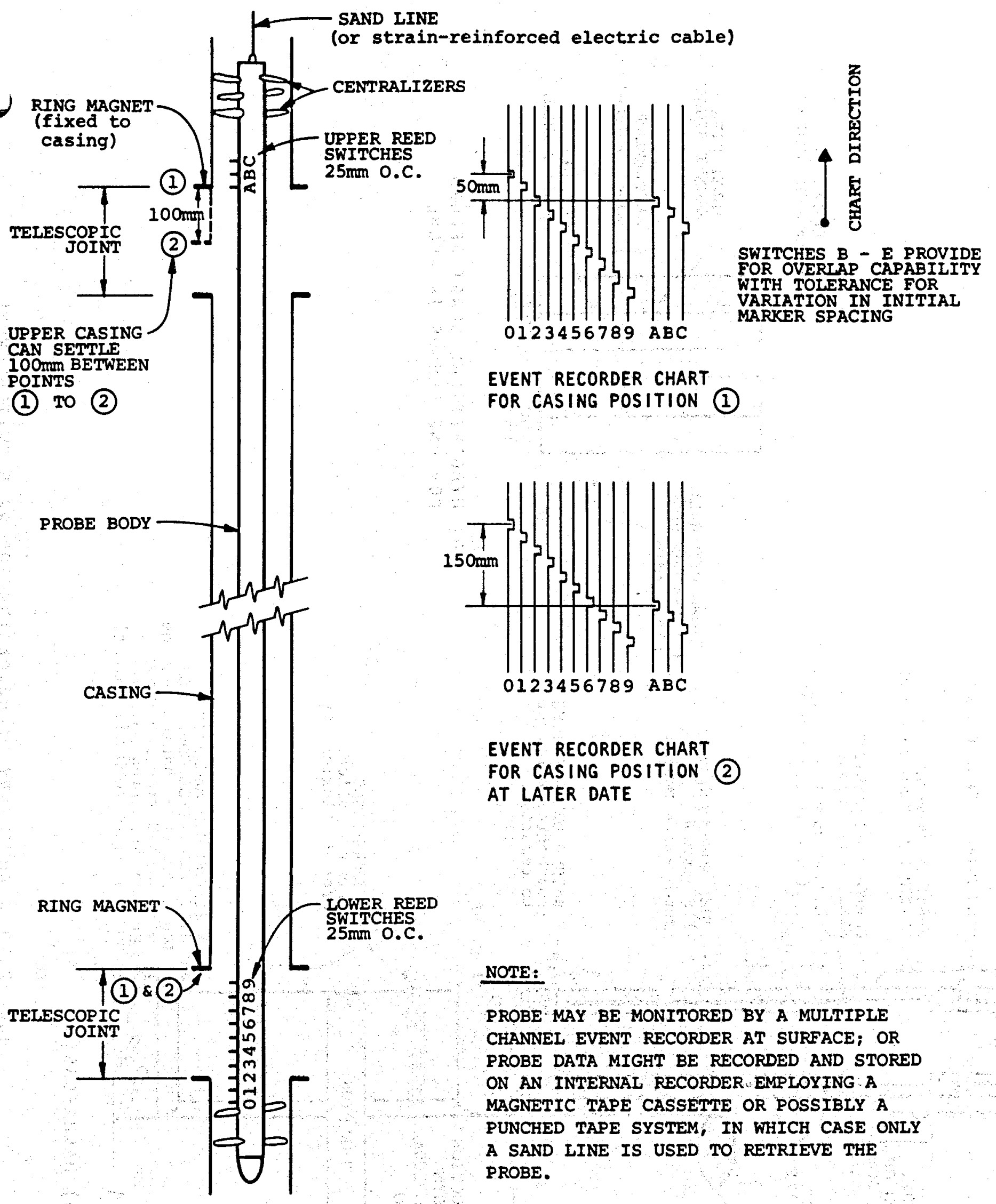

Figure 2.12 - MULTI-SENSOR REED SWITCH PROBE 


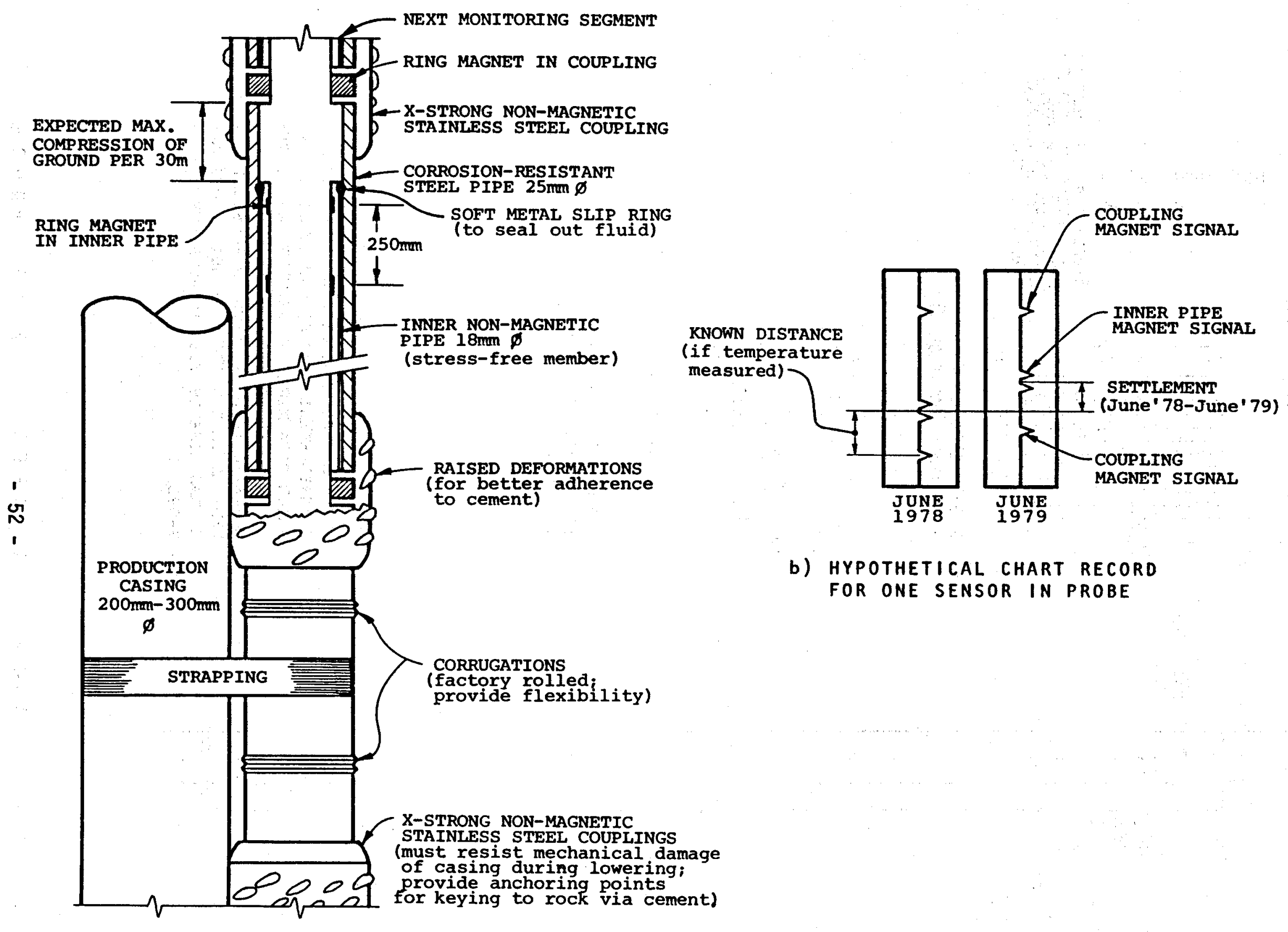

a) MAGNA-CASING 


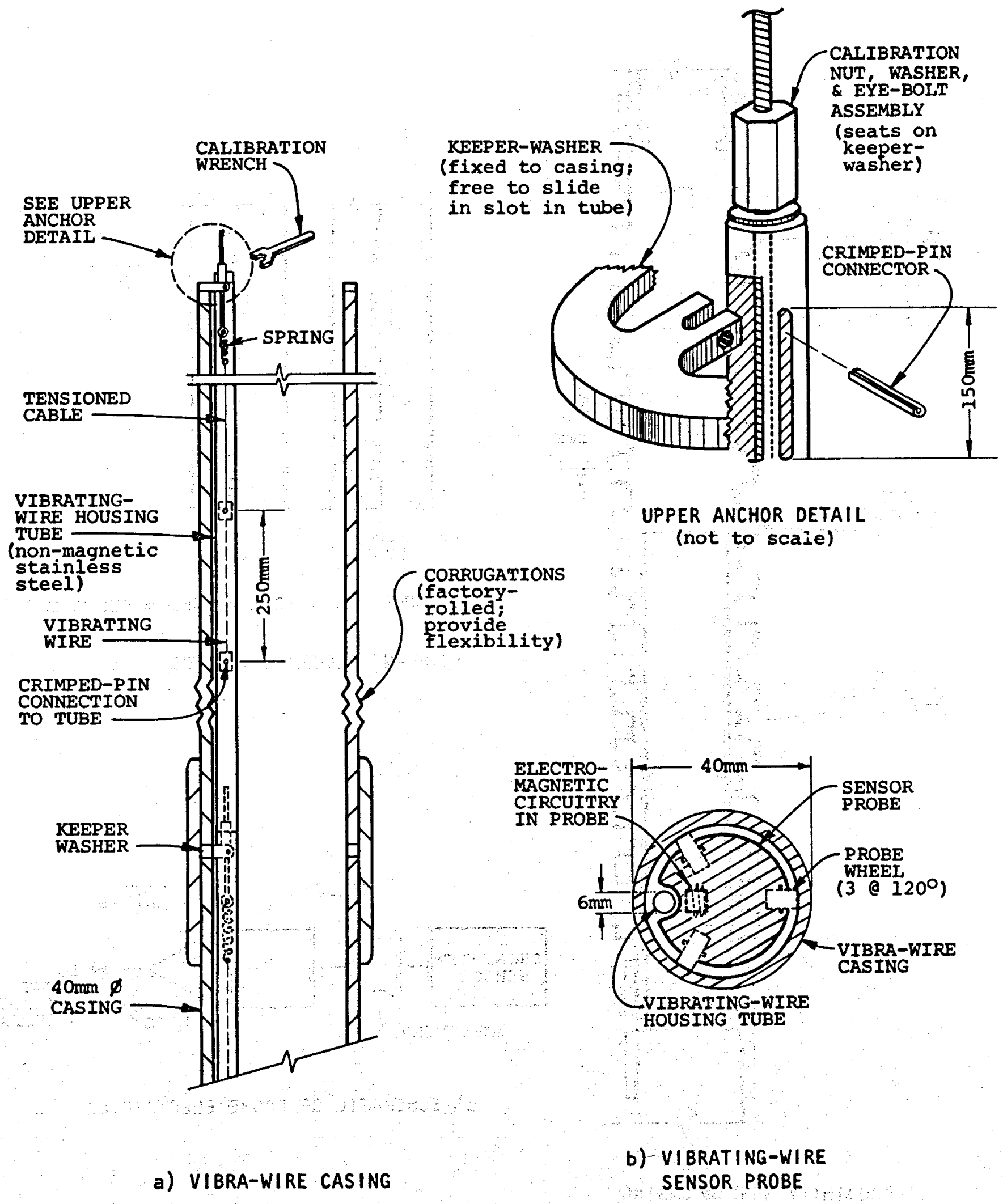

Figure $2.14-V$ I BRATING WIRE METHOD 


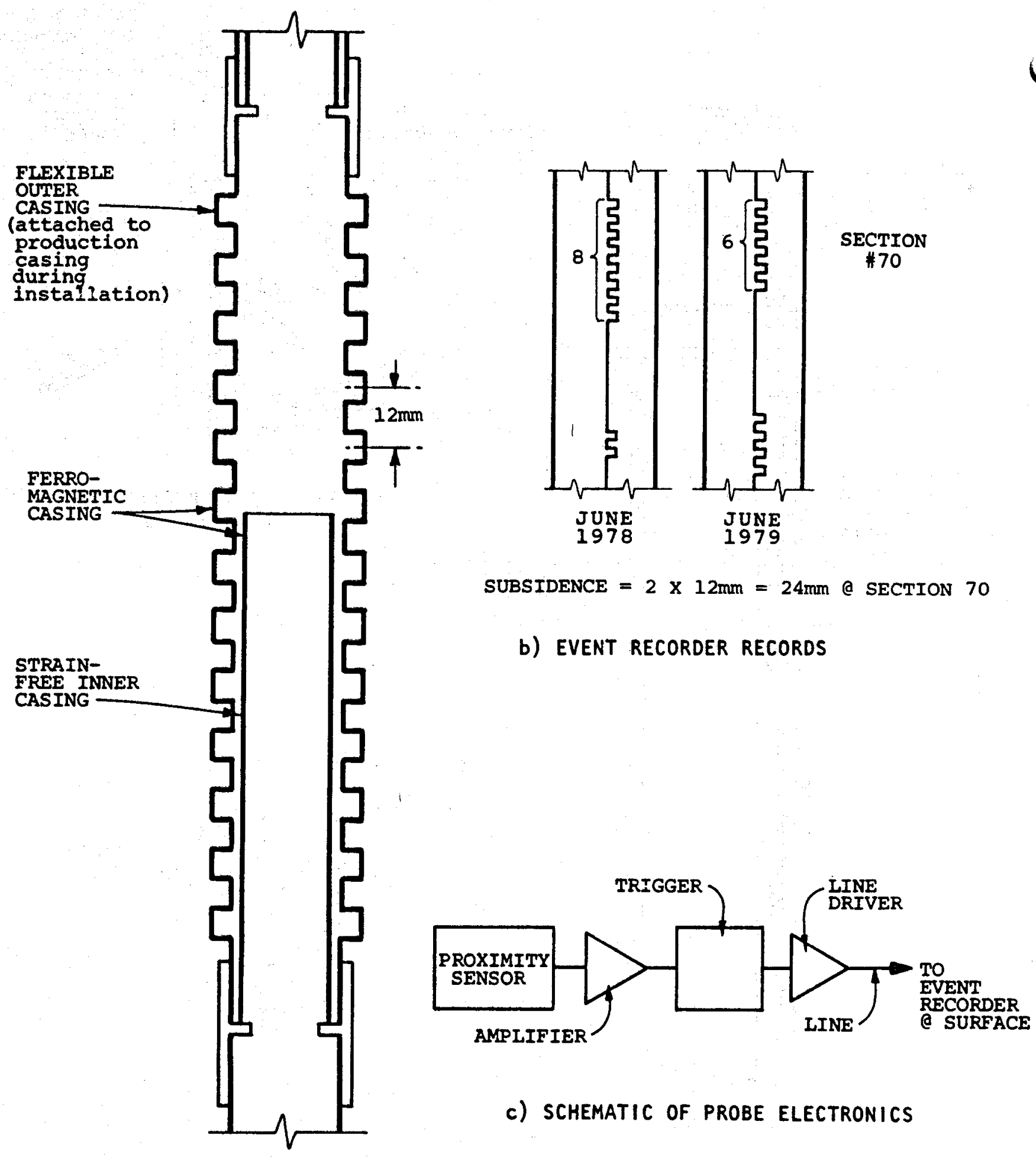

a) PROXIMITY SENSOR CASING

Figure 2.15 - PROXIMITY SENSOR PROBE METHOD 


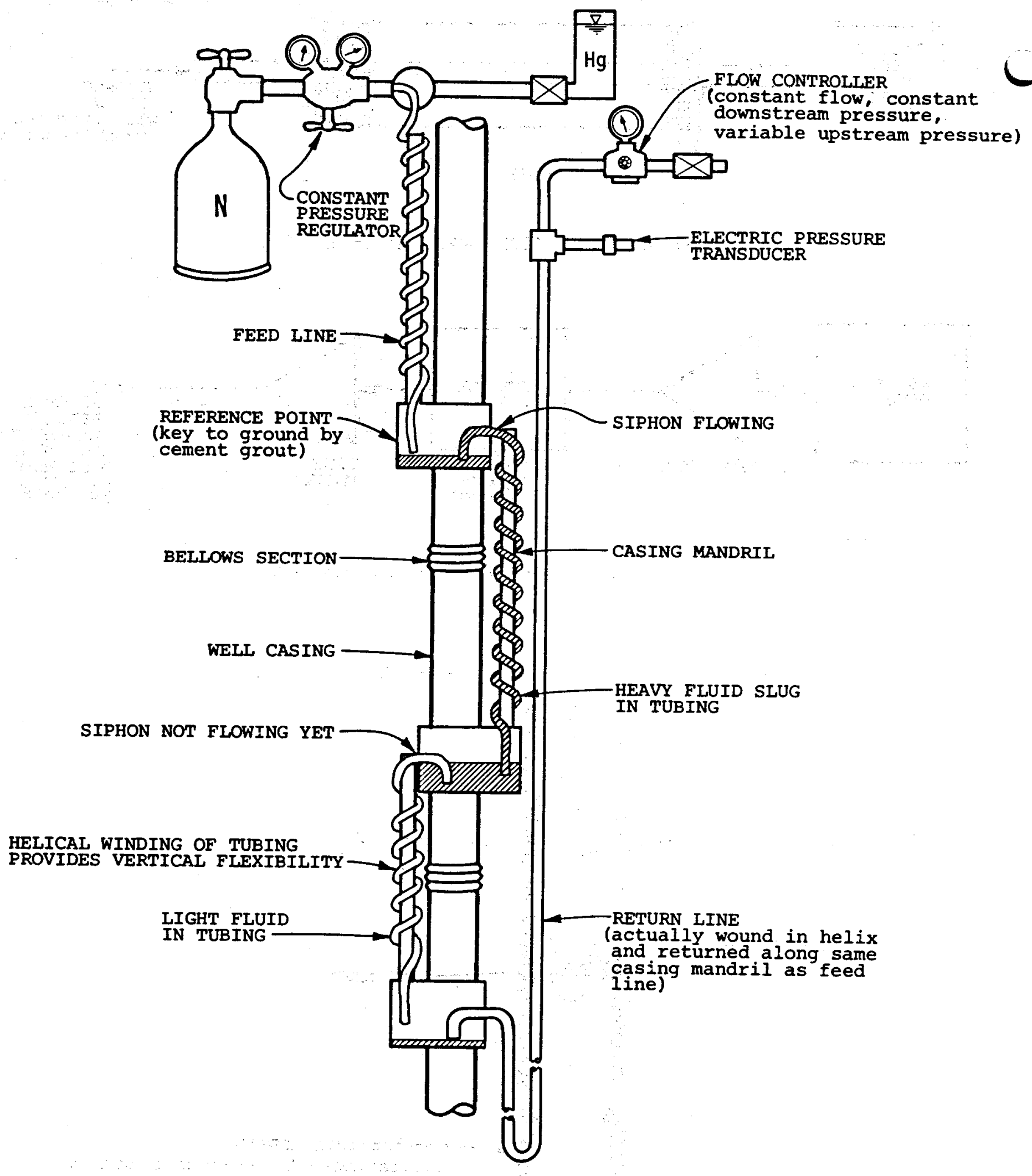

(a) SCHEMATIC OF DOUBLE FLUID SETTLEMENT DEVICE

Figure 2.17 - DOUBLE FLUID SETTLEMENT DEVICE METHOD 
(1)

(2)

(3)

(4)
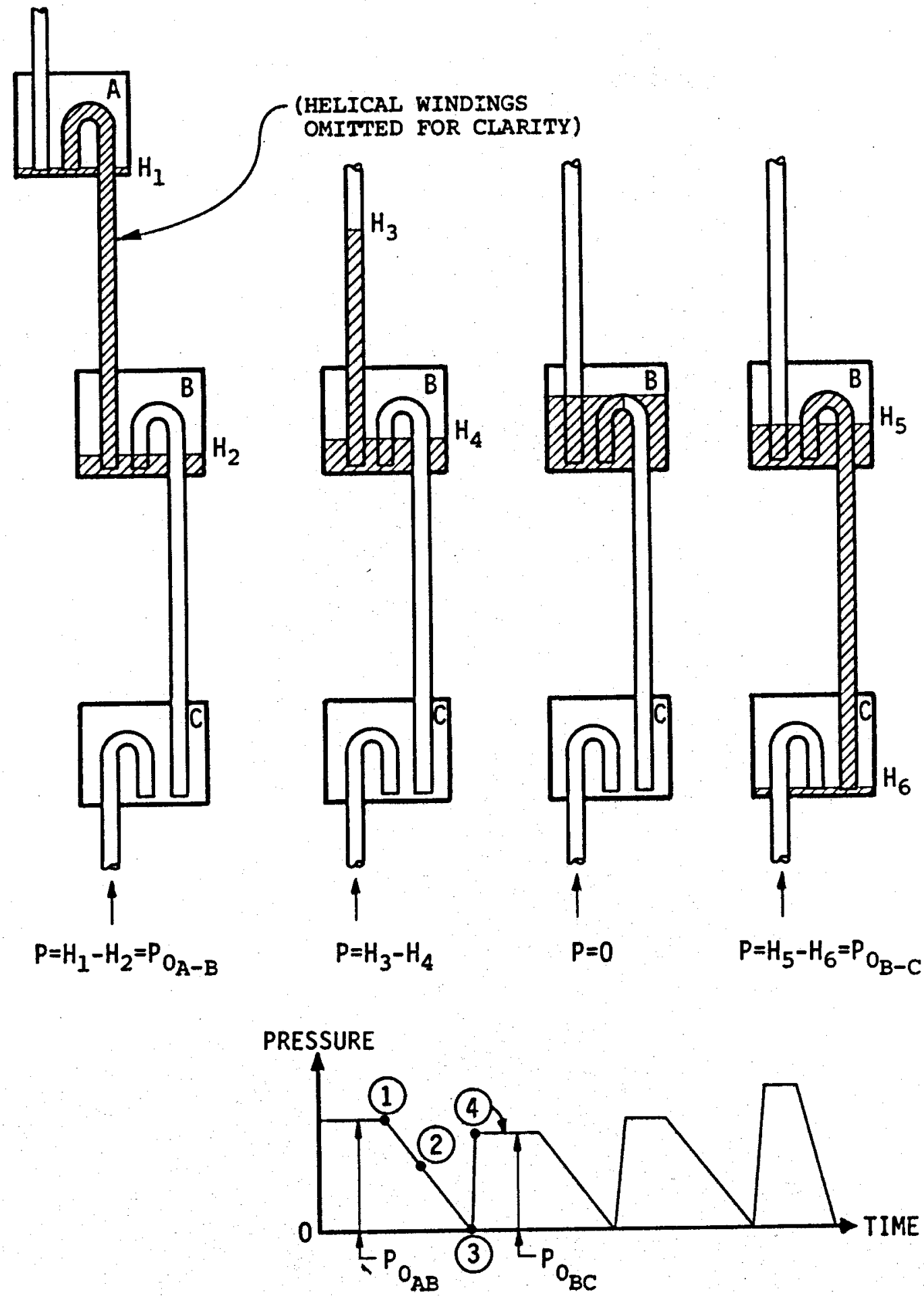

NOTE:

(b) SYSTEM OPERATION SHOWING READOUT

EACH INTERVAL BETWEEN REFERENCE POINTS HAS A UNIQUE VALUE OF PO INITIALLY; AS SUBSIDENCE OCCURS THE VALUE OF $P_{0}$ WILL DECREASE. THE AMOUNT OF SUBSIDENCE MAY BE DIRECTLY CALCULATED FROM THE CHANGE IN $P_{0}:$ SUBSIDENCE $=\Delta P_{0} /\left(\gamma_{\mathrm{HG}}-\gamma_{\mathrm{N}}\right)$

Figure 2.17 - DOUBLE FLUID SETTLEMENT DEVICE METHOD (CONT'D) 
Woodward.Clyde Consultants

3.0 RECOMMENDED SUBSIDENCE MEASUREMENT INSTRUMENTS 


\subsection{RECOMMENDED SUBSIDENCE MEASUREMENT INSTRUMENTS}

\subsection{COMPARATIVE RATING OF EXISTING, IMPROVED AND NEW INSTRUMENTS}

\subsubsection{Promising Extensometers for Further Development}

In the Interim Reports prepared for this project, numerous instruments were discussed. In each report, the most promising of the instruments considered were identified by use of a rating procedure. Developmental work and testing can proceed on only a few of these instruments. To identify those instrument systems most likely to monitor geothermal subsidence movements successfully, the top instruments from each of the interim reports are comparatively evaluated in this section. The rating system for this purpose is adapted mostly from the two rating systems al ready used in this study. In Interim Report No. 1, a rating system was developed to evaluate the instrument's ability to meet basic monitoring objectives (accuracy, depth, etc.) and basic environmental requirements (corrosion, scaling, etc.). 'The rating system in Interim Report No. 4 examined in more detail certain technical problems such as the modifications to well casing installation. The present, comprehensive rating system is shown in Table 3.1. The instruments are rated in Table 3.2. The extensometers identified as meriting further development are:

- TRIPLE SENSOR INDUCTION PROBE (with casing collar markers)

- TRIPLE SENSOR GAMMA RAY DECTECTOR PROBE (with radioactive markers)

- TRIPLE SENSOR REED SWITCH PROBE (wi th magnet markers)

- TRIPLE SENSOR OSCILLATOR-TYPE MAGNET DETECTOR PROBE (with magnet markers)

All are designed for use in cased wells and will function best if the well casing incorporates slip couplings or bellows sections. The gamma ray detector probe may also be used in unlined holes. Table 3.3 summarizes the basic specifications for these instruments.

\subsubsection{Promising Inclinometers for Future Development}

As work proceeded on this study, it became increasingly apparent that to reach the primary goal of this project, the development of an instrument to monitor vertical displacements, many problems had to be overcome. At the same time it became clear that the secondary aim, measurement of horizontal movements, was perhaps even more difficult to achieve. First, an inclinometer will experience the same temperature, corrosion, scaling and electronics problems that a settlement probe must cope with. It must be capable of measuring not only inclination, but also orientation and depth 
for accurate results. In fact, to ensure that horizontal measurements are repeatedly made at the same locations, the inclinometer may incorporate a probe-type settlement sensor to detect downhole markers. The depth of the horizontal measurement could then be determined by looking up the depths of the markers in the vertical displacement survey. Secondly, the horizontal movements due to subsidence are generally much smaller than vertical movements, requiring relatively more sensitive sensors. Because of these measurement problems and because the measurement of subsurface horizontal movements is not commonly performed in subsidence engineering studies, in this study extensometer development has been emphasized over inclinometer development.

Promising inclinometers incorporating mechanical and materials improvements were identified in Interim Report No. 2. Some of the electronics problems can be resolved using components and techniques discussed in Interim Report No. 3. These inclinometer systems:

- SONDE-TYPE BOREHOLE INCLINOMETER

(with servo-accelerometer and gyrocompass; modified)

- SONDE-TYPE BOREHOLE INCLINOMETER

(with cantilevered pendulum, vibrating wire strain gauge sensors, and gyrocompass; modified)

merit further development. However, we feel that such work should not begin until the major developmental problems in vertical subsidence monitor systems have beenr resolved. Consequently, these instruments are not further rated or discussed in this report.

\subsection{REVIEW OF SELECTED EXTENSOMETER SYSTEMS}

The rating procedures identified four instrument systems for further development. Recommendations for the developmental process are presented in Section 4.0. A discussion of the basic features of each of these candidate instrument systems is presented below.

\subsubsection{General Considerations}

All of the systems proposed for further development use probes containing three sensors that detect downhole markers. The following comments apply to all of them.

Sensor and Marker Spacing: In general it appears to be most convenient to incorporate the downhole markers into the casing couplings. The distance between couplings is typically 10-15 m; in design of the probe, the top and bottom sensors should be separated by the typical coupling spacing for a given geothermal field. The middle sensor should be one-third that distance from the top (or lower) sensor. The system may be designed so that the top (or lower) and middle sensors share a transmission channel since they will not intercept a marker simultaneously. 
Casing: of the four proposed systems, all except the radiation sensor system must operate in cased holes. For measurement purposes; the most accurate results will be obtained if the well casing is keyed to the producing formation. As noted previously we can then then monitor casing compression as a measure of formation compaction. This keying effect can be achieved with a well casing that is cemented, perforated as needed and incorporates slip couplings. Accurate results may al so be obtained using a gravel-packed slotted liner with slip couplings. In addition, in the Gulf Coast geopressured formations, the use of slip couplings will prevent loss of the installation if large compaction movements occur.

Unfortunately, slip couplings are not yet available for geothermal use. They should be considered a high priority development item. Without slip couplings, satisfactory compaction monitoring of somewhat lower sensitivity is still possible in liquid-dominated areas as long as the casing is cemented or gravel-packed. In these areas slip couplings may not be needed to prevent casing damage, since compaction is expected to be small due to reinjection of brine into the formation. Where slotted liners are used without a gravel pack, results may be less accurate. If the ground/liner adhesion is poor, it is still possible to measure the average compaction between the end of the last cemented string and the bottom of the hole. The use of at least slotted liner in the producing zone is desirable because markers can be conveniently and reliably incorporated into the couplings. For monitoring in unlined production zones, the only viable system appears to be the use of a triple sensor gamma-ray detector probe with radioactive markers shot into the formation.

In-Situ Calibration: The distance between the sensors is considered to be a know quantity for analysis purposes: Downhole temperature must be measured in the probe body so that the distance between sensors, measured at the surface, can be corrected for temperature-induced changes in probe length in the well. Once this value is determined, the distances between markers can be scaled from the strip chart record, as shown in Figure 2.4 of Section 2. This method makes the assumption that the probe velocity is constant between adjacent markers. This assumption seems to be acceptable.

Insulation and Refrigeration: One of the first research priorities will be to determine if insulation and refrigeration can be used to maintain a constant temperature of $100^{\circ} \mathrm{C}$ or less in the probe's instrument chamber. This would simplify circuit design of many signal conditioning equipment elements. However, the basic sensors, such as the coils of the induction sensor or the reed switches, still may have to be outside the insulation and exposed to $300^{\circ} \mathrm{C}$ in order to have adequate sensitivity.

\subsubsection{Triple Sensor Induction Sensor Probe}

This instrument uses a probe containing three induction sensors. The casing couplings serve as markers and are detected by the probe; refer to Figure 3.1a. Induction sensors are promising because marker degradation with time is not likely to be a problem and because design of the sensors appears feasible with high temperature materials and components. Casing 
collar locators, a similar instrument which operates on the induction principle using a permanent magnet in lieu of an exciter coil, have been used successfuily in several subsidence monitoring programs in oil and gas wells at lower temperatures (Allen,1969; deLoos, 1973):

The Sensors: Induction sensors used for geophysical logging typically consist of an exciter and a pick-up coil. The exciter coil induces eddy currents in the well casing. The eddy currents in turn induce an alternating voltage in the pick-up coil. The phase of this signal is shifted from that of the exciter coil. The phase shift is transmitted to the surface by appropriate signal conditioning equipment (Fig. 3.1b).

The coupling between the exciter and pick-up coils depends on the metal thickness, its magnetic permeability, its electrical resistivity and the gap between the sensor and the metal. Wi th suitable centering devices, the last factor is kept constant. A typical signal from a casing log is shown on Figure 3.1c.

The Markers: The casing couplings (collars) serve as markers. The increased thickness of metal at the coupling is detected by the induction sensor. At Gronigen (deLoos, 1973), it was found that certain coupling designs give sharper signals than others. For subsidence monitoring, slip couplings are reconmended. It is probable that the thickness of metal necessary to give such couplings adequate strength would al so provide a good signal though the signal shape may change as the coupling telescopes during subsidence.

Development Needs: Current induction sensors are not designed to operate at $300^{\circ} \mathrm{C}$. Because of the decreased magnetic permeability of coils and the inceased resistivity of the well casing at these temperatures, some consideration will have to be given to adequate coupling of the coils via the well casing wi thout direct coupling. Downhole signal conditioning typically includes an oscillator for the exciter coil and electronics to amplify and transmit the pick-up coil signal by modulating a suitable carrier. The signal conditioning equipment can probably be designed with high temperature components.

The signal conditioning equipment must be designed to provide a clear signal even if corrosion or scaling occurs. Induction sensors detect loss of metal as well as casing collars. Severe corrosion may make it difficult to identify the signal due to the casing collars, as shown in figure 3.1d. A signal conditioning system, which transmits a 0-1 signal where 1 represents an event above a certain threshold value, is probably not recommended here. The risk is that a deeply corroded zone might register as an event. A related problem is caused by scaling. A coating of low permeability high resistivity scale may reduce the coupling between the exciter and pick-up coils and lessen the sensitivity of the system. 


\subsubsection{Triple Sensor Reed Switch Probe}

This instrument system uses a probe containing three reed switch sensors. Magnet markers installed downhole are detected by the probe. Refer to Figure 3.2a. Reed switch sensors are promising because no downhole signal conditioning is required and the output is an "on-off" signal as a marker is crossed.

The Sensor: Reed switches are made of two thin reeds of high magnetic permeability in an evacuated glass housing ( $F$ igure $3.2 b$ ). In the presence of a magnetic field, the reeds contact and close an electrical circuit. The reed switches thus provide an on-off signal. No downhole signal conditioning is necessary. The position of the reed switch (open or closed) may be detected at the surface by current measurement. However, if stray currents are a problem, the 0.1 amp allowable current through a reed switch may be hard to detect. In that case reflectometry may be used by monitoring the phase change between open and closed positions of the switch. A temperature sensor must al so be included in the probe to determine its length.

The Markers: Rare earth or possibly Alnico magnets may be used. They require non-magnetic stainless steel (annealed) backing to concentrate flux in the center of the ring, minimizing flux through the well casing. The magnets may be factory installed in (siip) couplings (Fig. 3.2c). To reduce the reluctance between the magnet and the reed switch, expendable leaf springs and a core of soft iron may be incorporated into the probe (Figure 2.2d).

Development Requirements: The key development is to determine whether existing reed switches will close at $300^{\circ} \mathrm{C}$. If not, a suitable reed switch can probably be developed. Factors in the development process may include selection of a high permeability material suitable for reeds at $300^{\circ} \mathrm{C}$, selection of a non-magnetic housing with expansion characteristics compatible with the reed material, and design of a seal between the reeds and housing.

Rare earth magnets have adequate magnetic strength at this temperature. A suitable housing which can be welded to a well casing coupling must be designed which minimizes flux in the casing, protects the magnet from corrosion and from mechanical damage, and prevents corrosion between the magnet and $i$ ts housing and between the housing and the coupling materials.

\subsubsection{Triple Sensor Oscillator-Type Magnet Detector System}

This instrument system uses a probe containing three oscillator-type magnet detectors. Magnet markers installed downhole are detected by the probe. Refer to Figure 3.3a. This system offers promise because it is more sensitive than a reed switch and thus more likely to give a good signal at $300^{\circ} \mathrm{C}$, at which sensitivities of all magnetic systems are reduced. Its simple circuitry can probabiy be constructed from high temperature components and the frequency output is good for transmission over long electrical leads. 
The Sensors: An oscillator using a saturable core reactor can detect smat changes in a magnetic field. This sensor type is much more sensitive than a reed switch, increasing the probability that it will operate at $300^{\circ} \mathrm{C}$, at which all magnet detectors decrease in sensitivity.

The oscillator is formed from two transistors and an inductor with a high permeability core of $\mu$ metal (Figure $3.3 \mathrm{~b}$ ). The transistors come into play so that the frequency of the oscillator is proportional to the time required to saturate the core of the inductor. An external magnetic field will speed up or decrease the saturation rate and consequently a frequency change occurs. This frequency is directly related to the magnetic field strength along the inductor's longitudinal axis:

The Markers: Rare earth or possibly Alnico magnets incorporated into couplings are suitable, as discussed in Secton 3.2.3.

Development Requirements: Further research is required to assess the performance of $\mu$-metal at $300^{\circ} \mathrm{C}$. Newer alloys (such as HyMu-80) may be superior. Temperature alone will cause a frequency shift and its effect on the accuracy of marker detection must be assessed. The main components of the basic circuit and signal conditioning equipment can probably be developed from high temperature components, such as those listed in Table 2.20. To improve the sensitivity of the detector, the use of a soft iron probe core and leaf springs as discussed in Section 3.2 .3 should be evaluated.

The problems of magnetic marker design also require consideration, as discussed in Section 3.2 .3 .

\subsubsection{Triple-Sensor Gamma Ray Detector System}

This instrument system uses a probe containing three gamma ray detectors. Radioactive markers installed downhole are detected by the probe. Refer to Figure 3.4a. This system has been used by several researchers and in particular was refined to a high accuracy in the Netherlands (deloos, 1973) for use in gas wells. It offers promise because it may be used in both cased and unilined holes and because the radioactive markers require no special shielding, as do the magnets, for use in steel casing.

The Sensors: Conventional modern gamma ray detectors use scintillation counters with photomultiplier tubes or semiconductor crystals. These are unlikely to operate at geothermal temperatures. In fact, the refinements provided by such counters (i.e., an exact particle count) is unnecessary. A signal indicating the presence of a radioactive source is all that is needed. The Geiger-Muller (G-M) tube (Figure 3.4b), commonly used before 1950, may be adaptable to high temperature use. When a radioactive particle enters this device, the gas inside the tube is ionized. The ionized gas allows current to flow between a thin wire at the center of the tube and the outer shell under an applied voltage of around 800 volts. Current will continue to flow unless the discharge is suppressed or "quenched." Once quenched, the G-M tube is ready to detect the next gamma ray. Since, for settlement measurement, an exact count of the radiation particles is 
not needed, self-quenching types should be adequate. These contain a gas which is decomposed using energy that otherwise would have caused emission of electrons. The dimensions of the G-M tube should be selected so the tube intercepts enough $\gamma$-rays as each marker is passed to produce a clear signal.

The Markers: Radioactive bullets made of cobalt-60 (3-6 $\mu$ Curies; Sano, 1969) or Cesium 137 (100 uCuries; deLoos, 1973) have been shot into the formation around a cased well using a perforating gun. This technique is necessary if the hole is unlined. Radioactive bullet placement should be shallow to maximize signal strength, but deeper penetration will be needed in unlined holes and in boreholes with uncemented casing compared to cemented casing because of the possibility of wall slump. Radioactive markers have also been wel ded as "buttons" to casing (Sano, 1969).. In cased holes, the best approach may be to weld such buttons to the inside of casing couplings, prior to installation. Radioactive matierals require special handling and precautionary procedures for all field and shop personnel.

Development Requirement: The development of a G-M tube is probably more difficult than the development of the previously discussed sensors. In particular, ionizing and quenching gases must perform their functions over $20^{\circ}-300^{\circ} \mathrm{C}$. The associated signal conditioning equipment (Figure $3.4 \mathrm{C}$ ) can probably be designed from high temperature components.

The radioactive markers must be safe to handle in the field while providing an adequte signal. The radioactive material must be bound so that it resists corrosion and subsequent diffusion. In unlined holes, bullet penetration may be a problem. Consideration should be given to bullet configuration and gun design so that a uniform penetration can be achieved in all formations. The optimum penetration, adequate to embed the bullet securely while providing a clear signal, should also be assessed. 
TABLE 3.1

MODIFIED RATING SYSTEM

\begin{tabular}{|c|c|c|c|}
\hline \multirow[b]{2}{*}{ CATEGORY } & \multicolumn{3}{|c|}{ RATING } \\
\hline & 3 & 2 & 1 \\
\hline $\begin{array}{l}\text { WORKING } \\
\text { DEPTH }\end{array}$ & $\begin{array}{l}\text { Greater than or equal } \\
\text { to } 3 \mathrm{~km}\end{array}$ & $3 \mathrm{~km}$ to $300 \mathrm{~m}$ & less than $300 \mathrm{~m}$ \\
\hline $\begin{array}{l}\text { ABILITY } \\
\text { TO MAKE } \\
\text { INTERVAL } \\
\text { MEASURE- } \\
\text { MENT }\end{array}$ & $\begin{array}{l}\text { Many intervals in } \\
\text { one hole }\end{array}$ & $\begin{array}{l}2 \text { to } 5 \text { intervals } \\
\text { in one hole }\end{array}$ & $\begin{array}{l}\text { More than one } \\
\text { hole required }\end{array}$ \\
\hline $\begin{array}{c}\text { RELATIVE } \\
\text { SENSITIVITY } \\
\text { TO } \\
\text { GROUND } \\
\text { MOVEMENT }\end{array}$ & $\begin{array}{l}\text { Sensitive to movement } \\
\text { over short intervals } \\
\text { (100 } \mathrm{m} \text { or less) }\end{array}$ & $\begin{array}{l}\text { Sensitive to } \\
\text { movement between } \\
\text { widely spaced } \\
\text { reference } \\
\text { points }(>100-\mathrm{m})\end{array}$ & $\begin{array}{l}\text { Distance between } \\
\text { markers may not be } \\
\text { representative of } \\
\text { ground movement }\end{array}$ \\
\hline $\begin{array}{l}\text { NEED } \\
\text { FOR } \\
\text { CASING } \\
\text { MODIF- } \\
\text { ICATIONS }\end{array}$ & $\begin{array}{l}\text { Slip couplings or } \\
\text { bellows sections } \\
\text { only }\end{array}$ & Auxiliary casing & $\begin{array}{l}\text { Auxiliary casing } \\
\text { with special inserts }\end{array}$ \\
\hline $\begin{array}{l}\text { OPERATION } \\
\text { REQUIRE- } \\
\text { MENTS }\end{array}$ & $\begin{array}{l}\text { No access to } \\
\text { well required }\end{array}$ & $\begin{array}{l}\text { Requires special } \\
\text { fittings at } \\
\text { surface; some } \\
\text { interference } \\
\text { with fluid } \\
\text { production } \\
\text { during readout }\end{array}$ & $\begin{array}{l}\text { Substantial } \\
\text { interference with well } \\
\text { operation for readout; } \\
\text { well may have to be } \\
\text { taken out of production } \\
\text { for monitoring use }\end{array}$ \\
\hline $\begin{array}{c}\text { DEVELOPMENT } \\
\text { REQUIREMENTS } \\
\text { DUE TO } \\
\text { TEMPERATURE }\end{array}$ & $\begin{array}{l}\text { Current design } \\
\text { functions at } 300^{\circ} \mathrm{C}\end{array}$ & $\begin{array}{l}\text { High temperature } \\
\text { components avail- } \\
\text { able to construct } \\
\text { most of system; } \\
\text { Development of } \\
\text { remaining compo- } \\
\text { nents promising }\end{array}$ & $\begin{array}{l}\text { As } 2 \text {, but significant } \\
\text { developmenta } 1 \text { work } \\
\text { required for certain } \\
\text { components }\end{array}$ \\
\hline $\begin{array}{l}\text { SCALING } \\
\text { AND } \\
\text { CORROSION } \\
\text { POTENTIAL }\end{array}$ & $\begin{array}{l}\text { No mechanical parts } \\
\text { exposed. No parts } \\
\text { sensitive to corro- } \\
\text { ston downhole }\end{array}$ & $\begin{array}{l}\text { Wheels or moving } \\
\text { parts on tempo- } \\
\text { rary probes; } \\
\text { downhole magnets } \\
\text { used }\end{array}$ & $\begin{array}{l}\text { Permanent downhole } \\
\text { mechanical probe parts } \\
\text { or casing parts }\end{array}$ \\
\hline $\begin{array}{l}\text { DOWNHOLE } \\
\text { ELECTRONICS }\end{array}$ & None & Minimal & $\begin{array}{l}\text { Downhole signal } \\
\text { conditioning required }\end{array}$ \\
\hline
\end{tabular}


TABLE 3.2

INSTRUMENT SYSTEM RATING MATRIX

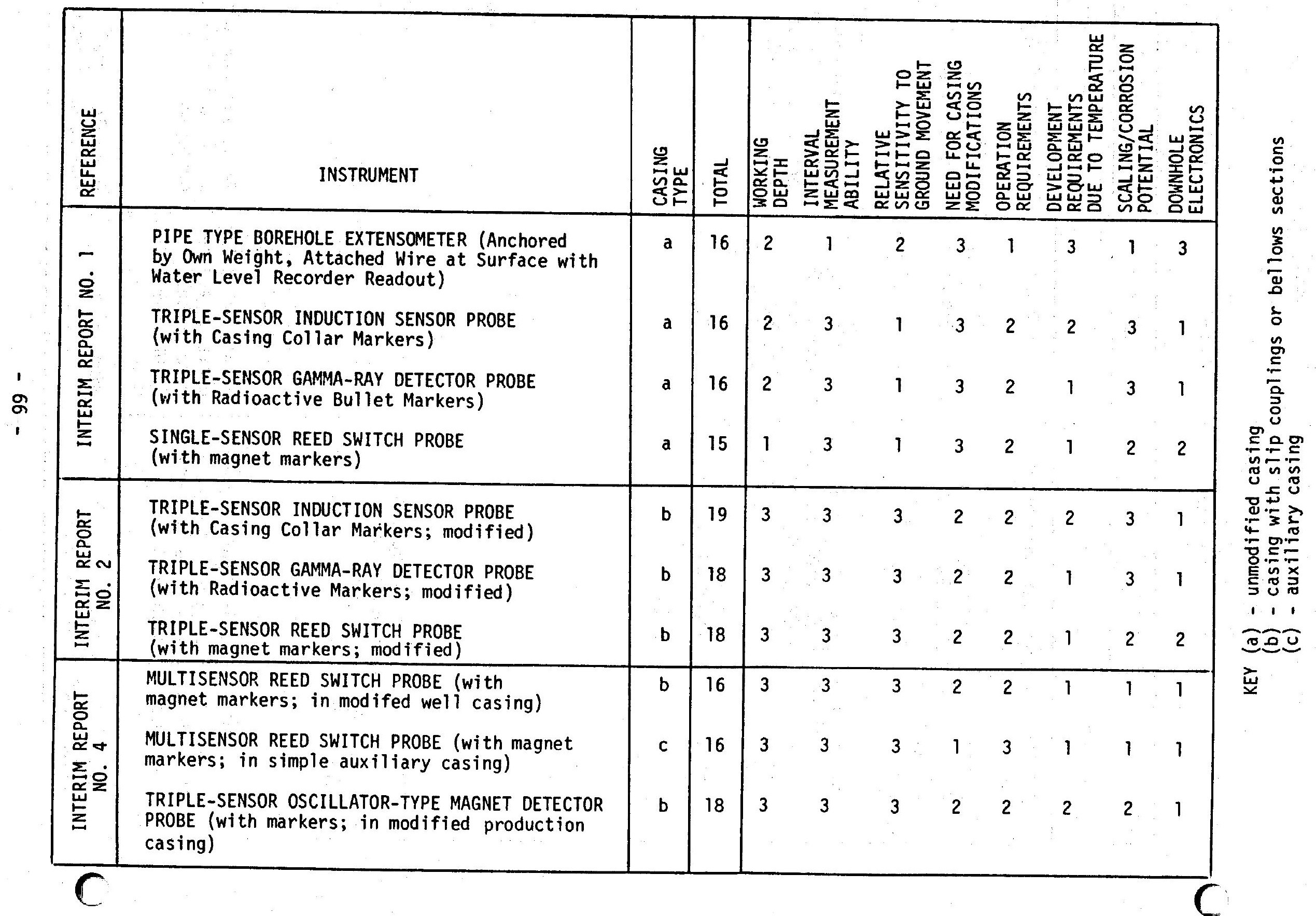


Woodward-Clyde Consultants

TABLE 3.3 - GENERAL SPECIFICATIONS FOR SELECTED INSTRUMENT SYSTEMS

\begin{tabular}{|c|c|c|c|c|}
\hline $\begin{array}{r}\text { INSTRUMENT } \\
\text { SYSTEM }\end{array}$ & $\begin{array}{l}\text { 3-Sensor } \\
\text { Induction } \\
\text { Probe }\end{array}$ & $\begin{array}{c}\text { 3-Sensor } \\
\gamma \text {-Ray Detector } \\
\text { Probe }\end{array}$ & $\begin{array}{c}\text { 3-Sensor } \\
\text { Reed Switch } \\
\text { Probe }\end{array}$ & $\begin{array}{c}\text { 3-Sensor } \\
\text { Oscillator-Type } \\
\text { Magnet Detector } \\
\text { Probe } \\
\end{array}$ \\
\hline Transducer & $\begin{array}{l}\text { Induction } \\
\text { Sensor }\end{array}$ & G-M tube & Reed Switch & $\begin{array}{l}\text { Oscillator-Type } \\
\text { Magnetometer }\end{array}$ \\
\hline $\begin{array}{l}\text { Location of } \\
\text { Transducer }\end{array}$ & $\begin{array}{l}3 \text { in Probe at } \\
0,10 \text { and } 15 \mathrm{~m} \\
\text { from end }\end{array}$ & $\begin{array}{l}3 \text { in Probe at } \\
0,10 \text { and } 15 \mathrm{~m} \\
\text { from end }\end{array}$ & $\begin{array}{l}3 \text { in Probe at } \\
0,10 \text { and } 15 \mathrm{~m} \\
\text { from end }\end{array}$ & $\begin{array}{l}3 \text { in Probe at } \\
0,10 \text { and } 15 \mathrm{~m} \\
\text { from end }\end{array}$ \\
\hline Marker & $\begin{array}{l}\text { Casing } \\
\text { Collars }\end{array}$ & $\begin{array}{l}\text { Radioactive } \\
\text { Bullets on } \\
\text { Collars or Shot } \\
\text { into Formation }\end{array}$ & $\begin{array}{l}\text { Magnets on } \\
\text { Casing Collars }\end{array}$ & $\begin{array}{l}\text { Magnets on } \\
\text { Casing Collars }\end{array}$ \\
\hline $\begin{array}{l}\text { Other } \\
\text { Ins truments } \\
\text { in Probe }\end{array}$ & $\begin{array}{l}\text { Temperature } \\
\text { Sensor }\end{array}$ & $\begin{array}{l}\text { Temperature } \\
\text { Sensor }\end{array}$ & $\begin{array}{l}\text { Temperature } \\
\text { Sensor }\end{array}$ & $\begin{array}{l}\text { Temperature } \\
\text { Sensor }\end{array}$ \\
\hline $\begin{array}{l}\text { Accuracy of } \\
\text { System } 1\end{array}$ & $\begin{array}{l} \pm 5 \mathrm{~mm} \text { over } \\
30 \mathrm{~m} \text { interval }\end{array}$ & $\begin{array}{l} \pm 5 \mathrm{~mm} \text { over } \\
30 \mathrm{~m} \text { interva } 1\end{array}$ & $\begin{array}{l} \pm 5 \mathrm{~mm} \text { over } \\
30 \mathrm{~m} \text { interval }\end{array}$ & $\begin{array}{l} \pm 5 \mathrm{~mm} \text { over } \\
30 \mathrm{~m} \text { interval }\end{array}$ \\
\hline $\begin{array}{l}\text { Temperature } \\
\text { Capability }\end{array}$ & $0-300^{\circ} \mathrm{C}$ & $0-300^{\circ} \mathrm{C}$ & $0-300^{\circ} \mathrm{C}$ & $0-300^{\circ} \mathrm{C}$ \\
\hline $\begin{array}{l}\text { Pressure } \\
\text { Capability }\end{array}$ & to $300 \mathrm{~kg} / \mathrm{cm}^{2}$ & to $300 \mathrm{~kg} / \mathrm{cm}^{2}$ & to $300 \mathrm{~kg} / \mathrm{cm}^{2}$ & to $300 \mathrm{~kg} / \mathrm{cm}^{2}$ \\
\hline
\end{tabular}

${ }^{1}$ For non-geopressured area use; these are recommended short-term goals. 


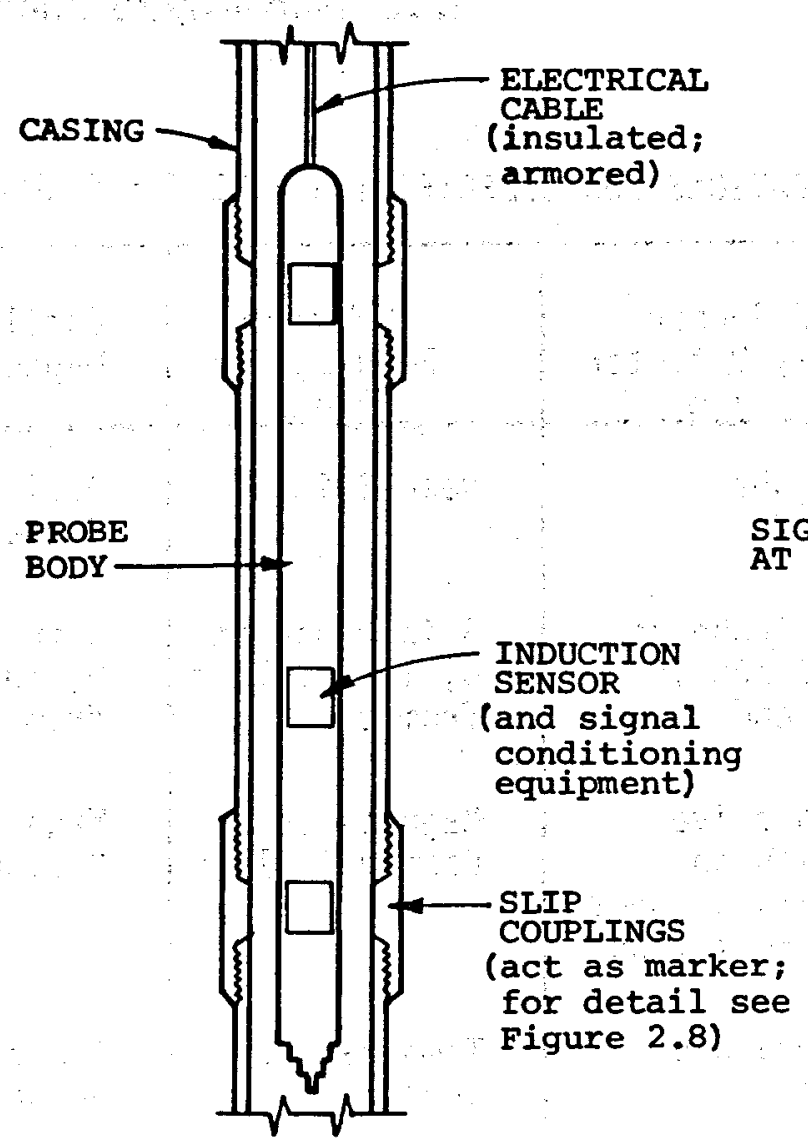

a) INDUCTION SENSOR PROBE SYSTEM

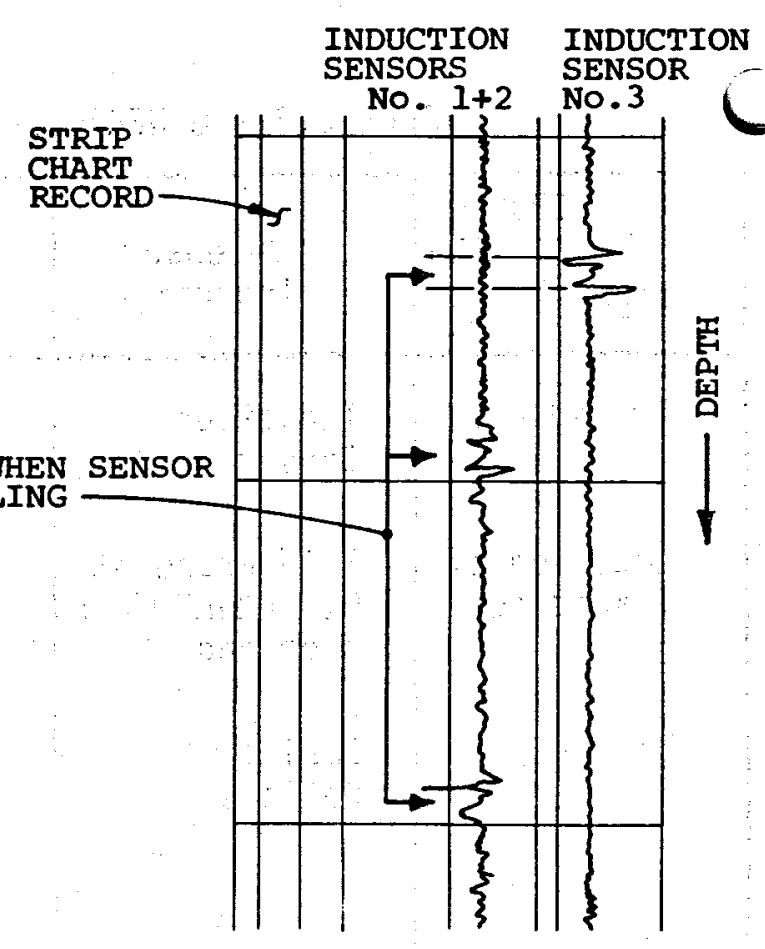

c) OUTPUT FROM TRIPLE SENSOR INDUCTION PROBE

(de Loos, 1973)
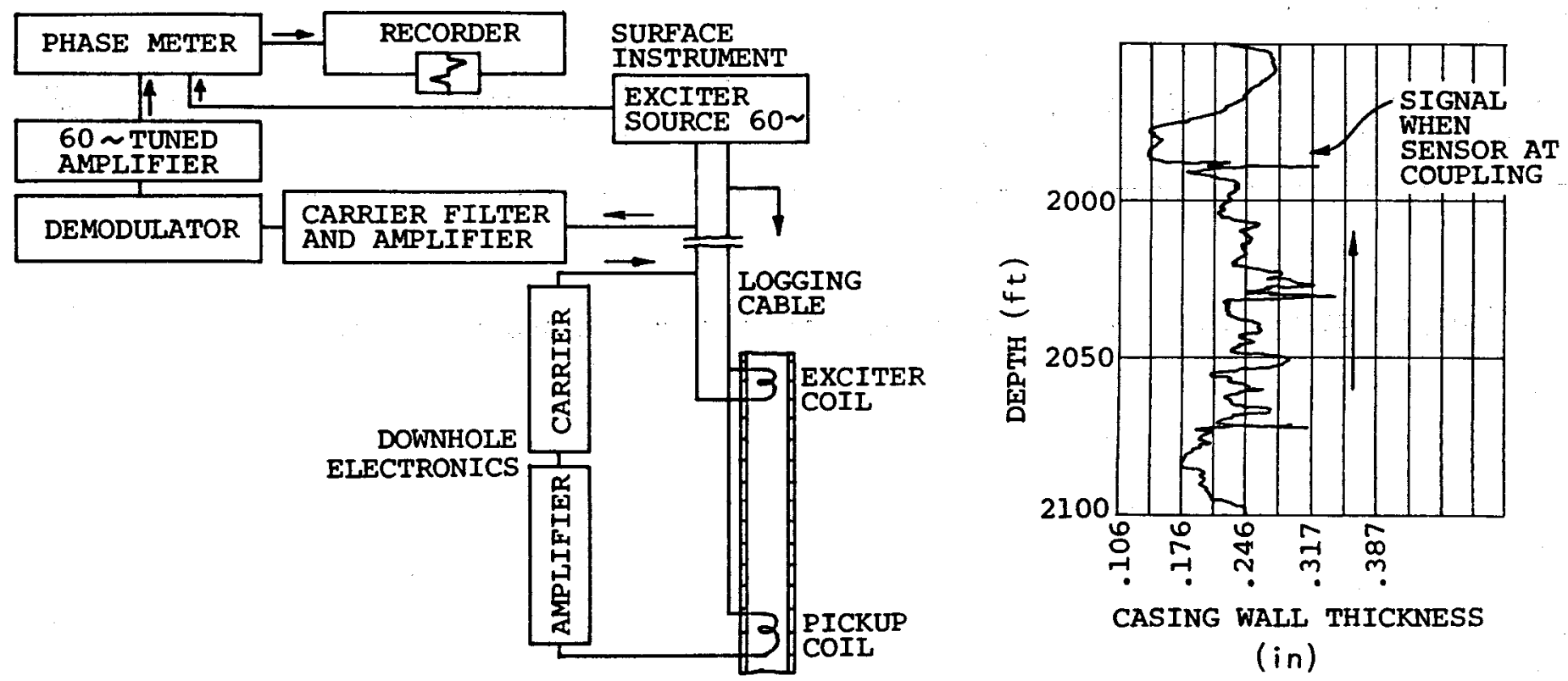

b) DIAGRAM OF INDUCTION SENSOR ELECTRONICS

(Edwards \& Stroud, 1964)

d) OUTPUT FROM SINGLE INDUCTION SENSOR IN CORRODED CASING (Edwards \& Stroud, 1964) 


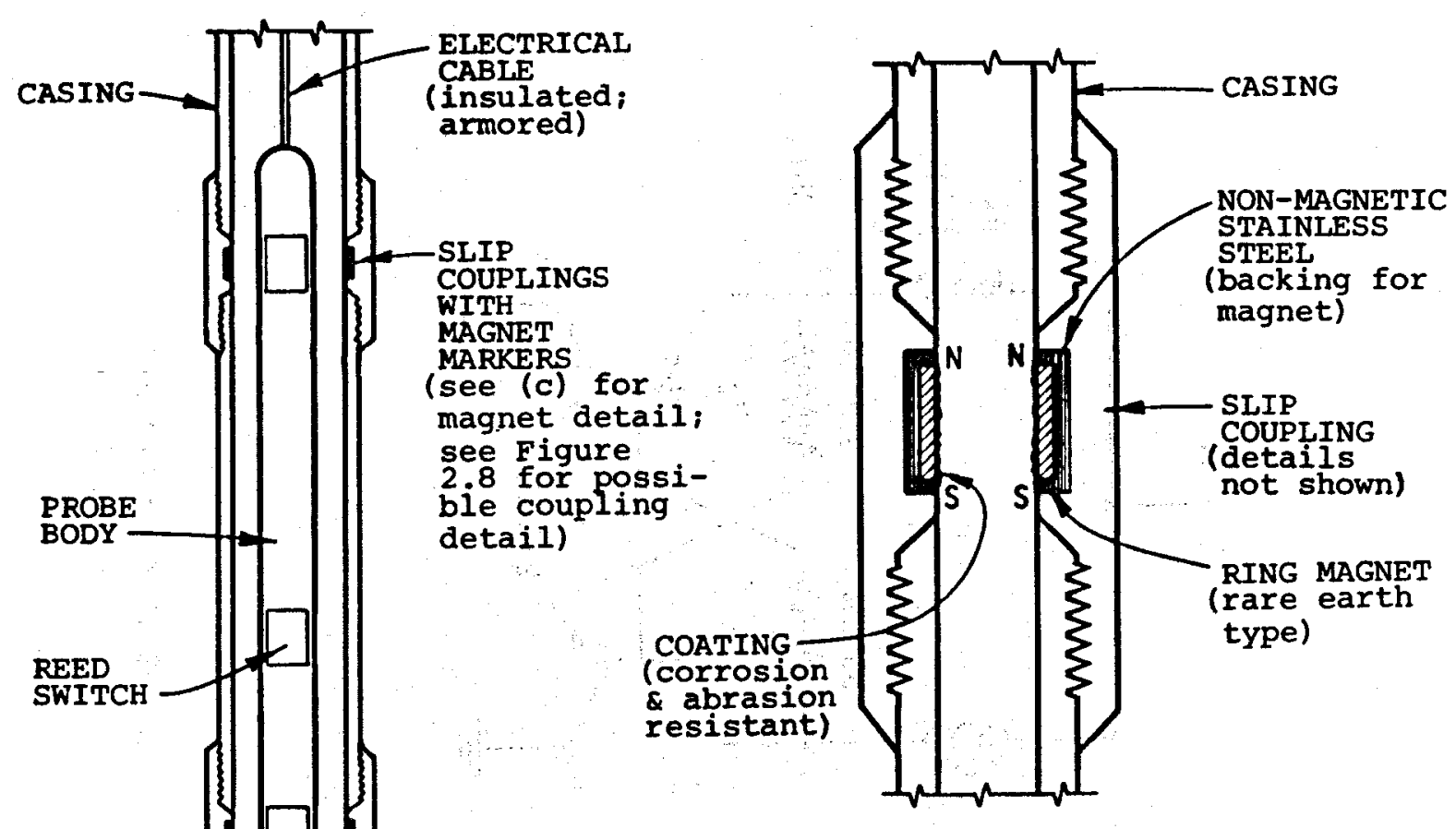

c) BASIC COMPONENTS OF MAGNET MARKER

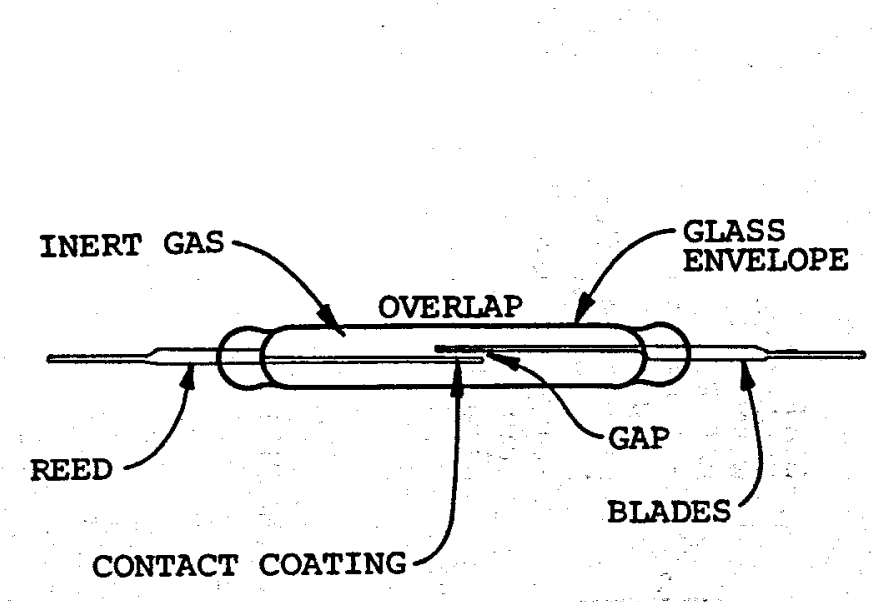

b) REED SWITCH COMPONENTS

a) REED SWITCH PROBE SYSTEM

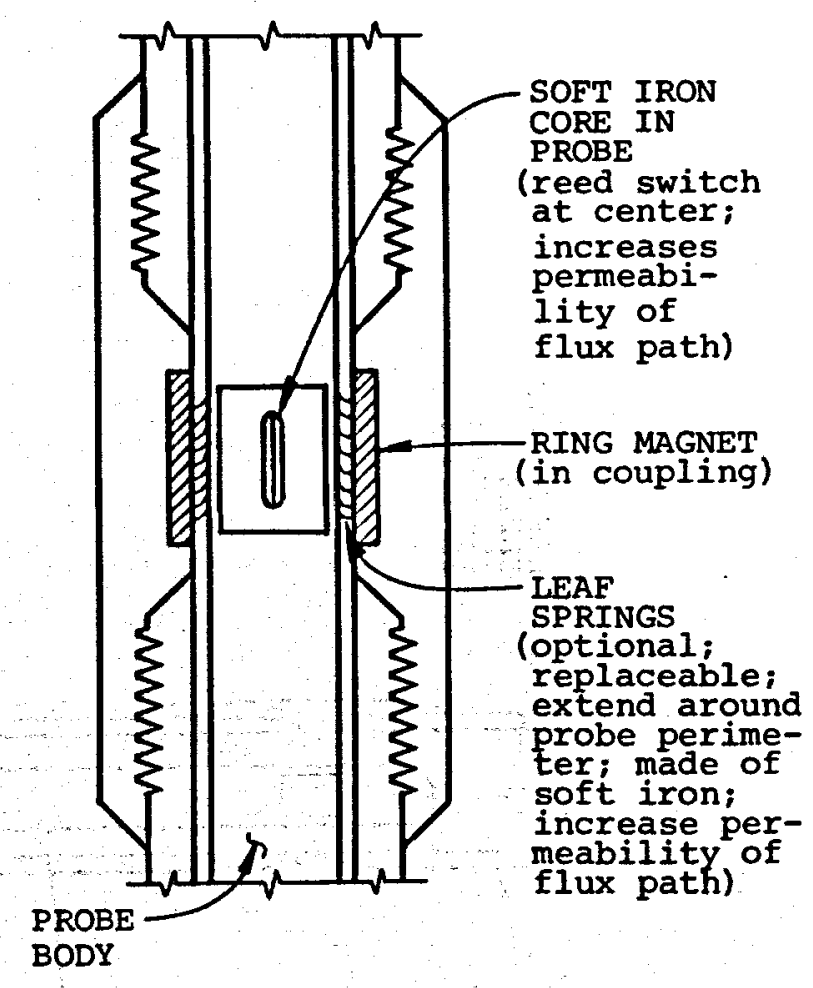

d) POSSIBLE DETAILS TO ENHANCE
SENSITIVITY OF SYSTEM

Figure 3.2 - TRIPLE SENSOR REED SWITCH PROBE METHOD 


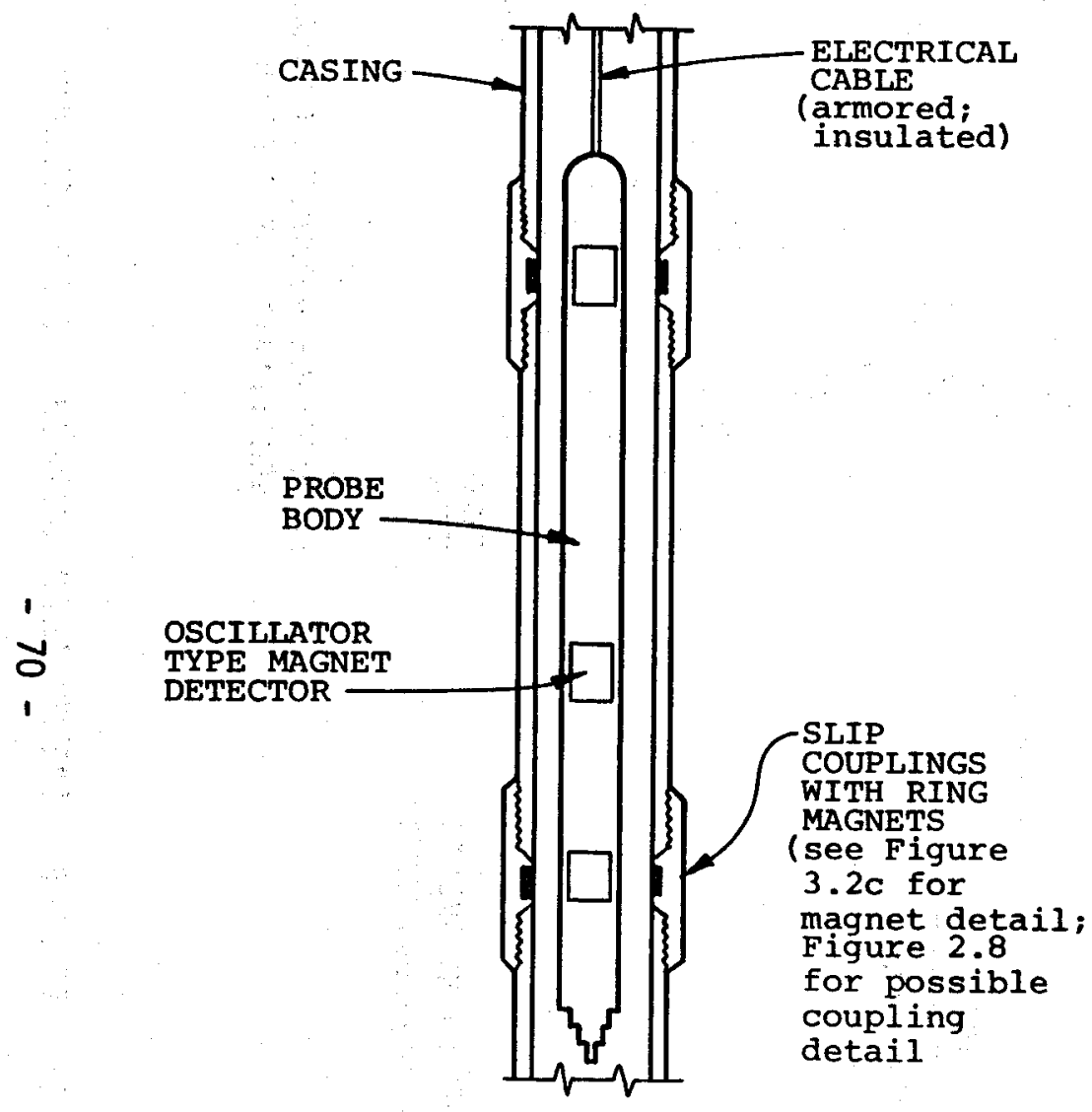

a) OSC ILLATOR-TYPE MAGNET DETECTOR SYSTEM

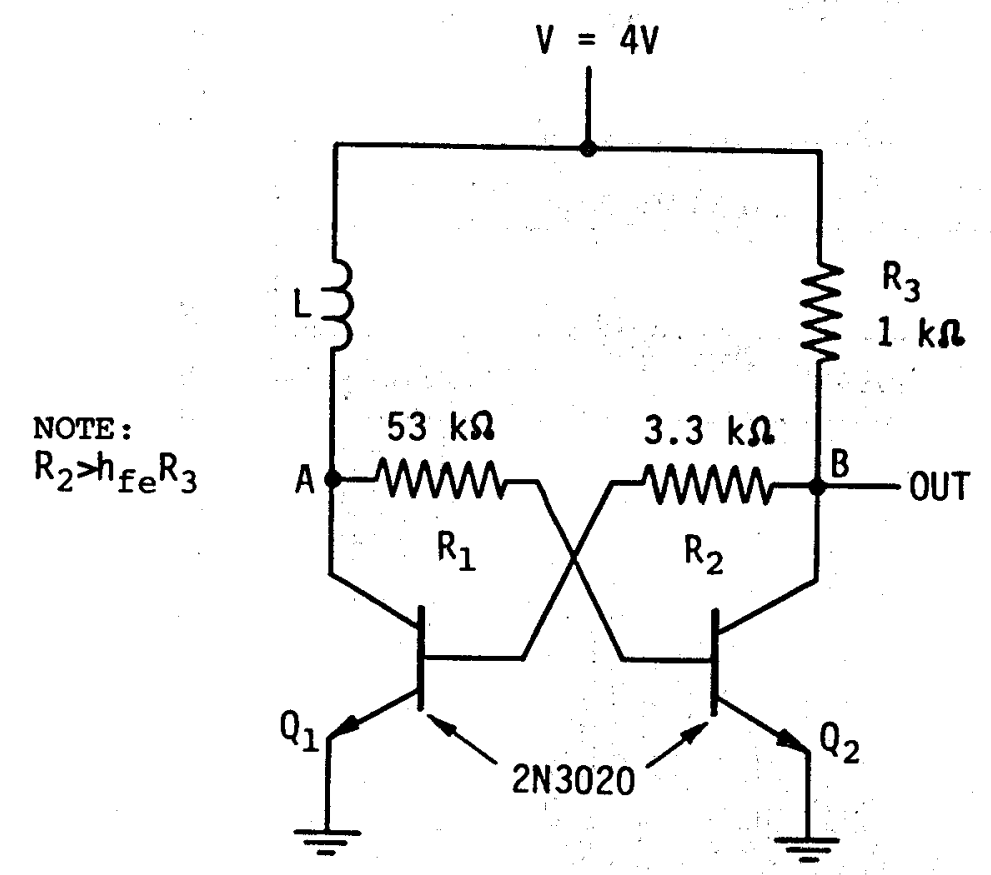

b) OSCILLATOR CIRCUIT

(Nair, 1977) 


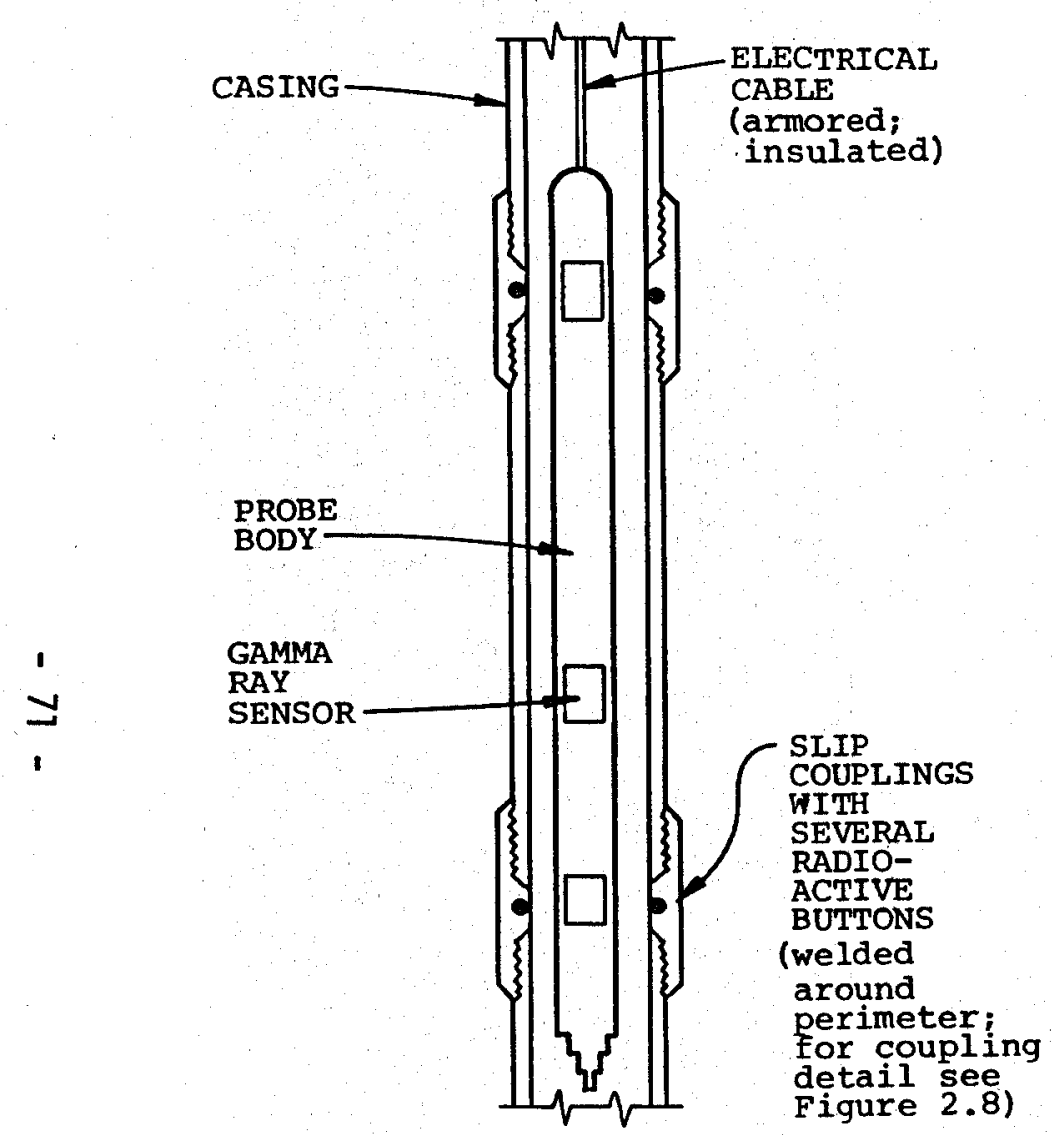

a) GAMMA RAY SENSOR PROBE SYSTEM

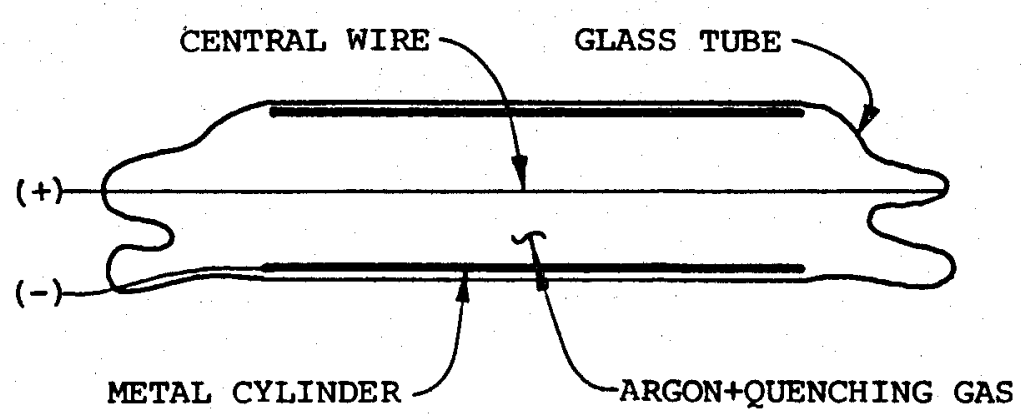

b) GEIGER-MULLER TUBE (after Glasstone, 1950)

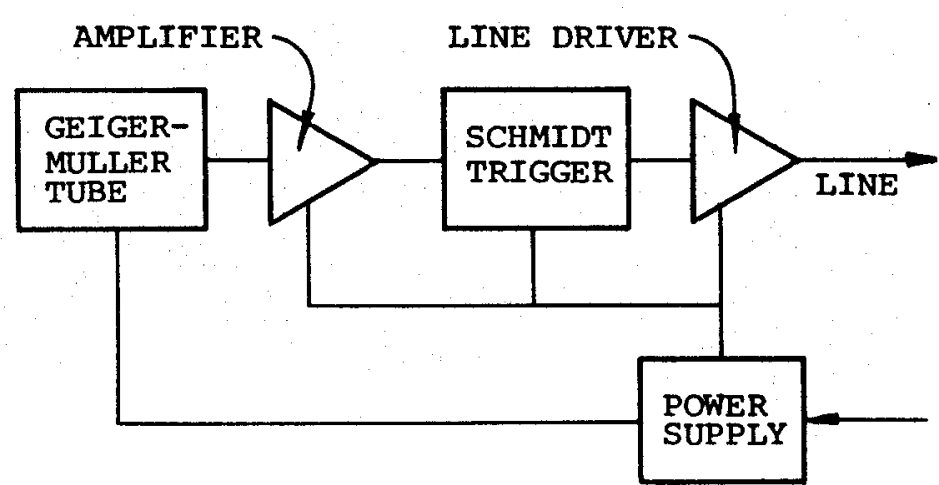

c) DOWNHOLE ELECTRONICS 


\section{Woodward.Clyde Consultants}

\subsection{RESEARCH PRIORITIES}

$-71 a-$ 


\subsection{RESEARCH PRIORITIES}

The first step toward the development of an instrument to monitor geothermal subsidence was undertaken with this research project (Geothermal Subsidence Research, Category 3, Project 1). With completion of this fifth and final report, we have assessed monitoring needs, reviewed relevant technology and selected four instrument systems for further development. These instrument systems, for monitoring vertical displacements above and within a geothermal reservoir, are presented in conceptual form in Section 3.0. The instrument designs include tested techniques and new technology from many disciplines, including electronics, materials science, geophysics, and the petroleum industry. Further development work is needed to determine which of the four instruments should be advanced to the prototype construction phase. This section discusses research strategies that will result in a working subsurface compaction monitoring instrument.

\subsection{PROPRIETARY INFORMATION}

Development of geothermal technology has been hampered by the limited market for it. According to a forecast developed by the Mitre Corporation (1978):
"The number of geothermal wel 1s... does not exceed 2000 in any year prior to 1990, and it does not exceed 500 before 1985. Compared to the 40,000-plus oil and gas wells now drilled annually in the U.S., the perceived market is likely to be too small to motivate industry to undertake major research, devel- opment and demonstration on its own in the near term. Govern- ment participation to accelerate the pace of geothermal specific well technology therefore appears justified."

Unfortunately, to date much of the research in the private sector, particularly with respect to scaling research and well logging technology, is proprietary. The authors of this report feel that if the government underwrites the development of geothermal subsidence monitor tools, it should be on a non-proprietary basis. Modifications and field testing can be performed more easily, and geothermal measurement technology can be advanced more quickly. The manufacturing and bench testing for instrument development should be open to any qualified precision mechanical or electrical fabricator, including well-logging service companies with manufacturing facilities.

No patentable items have been developed as a result of this study. However, if these conceptual instruments can be carried through to the prototype stage, patents may be possible. We feel that progress in geothermal well technology will be facilitated if the government owns all patents for which it financed the research. 


\subsection{POTENTIAL RESEARCHERS}

Figure 4.1 proposes a management structure, based on the assumption that instrument development will proceed under the overall management of Lawrence Berkeley Laboratory under the Geothermal Subsidence Research Program. A key feature of the management plan is the use of a geotechnical/subsidence engineering consultant to ensure that a workable, accurate subsidence monitoring system results from the research efforts of all the subcontractors. The roles of researchers with expertise in (1) geotechnical/subsidence engineering, (2) geophysical instrumentation engineering, and (3) geothermal well and production engineering are discussed below.

Geotechnical/Subsidence Engineer - Technical evaluation of research to ensure that the goals of the research program are being achieved is crucial to the successful development of a downhole compaction monitoring system. Civil engineers traditionally have managed technical projects involving other disciplines, such as mechanical and electrical engineering. With respect to the objective of developing a monitoring tool, geotechnical/civil engineers are experienced in the measurement of interest to this project. They understand the limitations of various direct measurement techniques and how all the components of a monitoring system interact to provide accurate data. Further, through the geotechnical engineers, technology transfer to and from related research in mining and waste disposal is facilitated.

LBL has excellent technical expertise, but may have limited staff availability to provide technical coordination and guidance to all of the researchers involved. We recommend that $L B L$ select a geotechnical/subsidence engineering consultant as a prime contractor to ensure that the research program achieves its objective of successful development of a compaction monitoring system (Figure 4.1). A more complicated but potentially satisfactory arrangement is shown in Figure 4.2 in which LBL manages al 1 contractual arrangements and provides overall technical direction. The geotechnical/subsidence engineering consultant is responsible for day-to-day technical coordination, essentialiy in the role of an LBL staff member, to achieve the project goals.

Geophysical Instrument Specialists - Development of a probe can probably best proceed under the direction of an organization experienced in geophysical instrumentation (e.g., Sandia Labs, Schlumberger, etc.). Because of the many technologies involved, including mechanical, electrical and materials engineering, they may find it appropriate to contract out portions of the work. Well logging companies should be involved in the development work because they are experienced in the costly and tricky procedures for running sensitive instruments through thousands feet of borehole. They also have support equipment for field operations, some of which may be directly and economically useful to the present project.

Geothermal Well and Production Engineering - A probe is only one component of a compaction monitoring system. Successful instrument system operation may require specialized couplings or casing, bullet-type markers, well head access glands, and means for controliting downhole scaling and corro- 
sion. Development of components for permanent downhole or wel lhead application might best proceed under overall supervision of a petroleum/geothemal engineer experienced in all phases of drilling and well completion. This management role could be filled by an energy (oil/geothermal) company or possibly a national laboratory such as Sandia or Los Alamos. Because of the wide variety of downhole and well lhead needs, several subcontractors may be involved, including designers and fabricators of tubing and couplings (e.g., Hydril, NL Atlas, etc.), well completion fluid suppliers, and well head assembly manufacturers.

\subsection{STRUCTURE OF FUTURE RESEARCH}

We recommend that future work be divided into two principal contracts (see Figure 4.3). In Project 2, the first of the two contracts, the four recommended instrument systems will undergo laboratory testing and development. From those results the most promising system will be selected for field testing in Project 3.

\subsubsection{Project 2 Research - Laboratory Testing}

This project comprises laboratory tests of the four key systems identified earlier in this report. The testing will evaluate materials, casing and coupling desing, sensor and signal conditioning design, and capabilities and other key features of the four candidate geothermal subsidence monitoring systems.

The project will require several areas of expertise. As noted in Section 4.2, it is recommended that the prime contractor be a firm experienced in geotechnical or subsidence engineering and monitoring. The prime contractor will coordinate the research in Project 2 and provide continual feedback to the subcontractors on the appropriateness of their findings to subsidence monitoring. Two main subcontractors will probably be required, as discussed in Section 4.1, one for probe development and one for well and logging equipment development. In the scheduling of probe development, it is recommended that research on the mechanical components begin six months in advance of the research on the electrical components because the capabilities of insulation and refrigeration systems will influence sensor development. In evaluating the sensor components, initial testing should determine the one most promising sensor type of the four recommended and further work should focus on improving probe performance. While probe development is underway, concurrent well and logging component development will concentrate on the design of slip couplings, well inead access glands, and logging cables. Overall, Project 2 should identify one of the four instrument systems and design, test and modify components to the point where a working field tool can be constructed in Project 3 .

The use of consultants by both the prime and subcontractors should be encouraged. For example, in the probe development subcontract, it is unlikely that one company has expertise in reed switch construction, hybrid circuit design and magnet technology together. However, it would probably be inefficient and cumbersome to award each of these study areas as separate contracts. Similarly, it may be useful for the prime contrac- 
tor to retain one or two consultants with expertise in oil well drilling and geothermal well operation. These consultants would advise on potential problems, operational requirements, and degree of acceptance by the geothermal industry for the proposed instruments systems.

At the completion of Project 2, the most promising instrument system from among the four tested will be selected for field testing in Project 3 .

\subsubsection{Project 3 Research, System Construction and Field Testing}

In this project, a full scale subsidence monitoring instrument system will be developed and tested. As in Project 2, the project work should be the responsibility of a single prime contractor, who should be experienced in geothermal or subsidence engineering and instrumentation. The function of the prime contractor is primarily inspection, coordination and administration. He will also be responsible for approving design changes as needed. The body of work for the project can be divided into three parts, as follows: (1) construct a prototype instrument, (2) concurrently furnish and install modified wel 1 casing and related equipment, and ( 3 ) then field test the prototype instrument system.

Before the letting of the subcontracts, an agreement with a geothermal operator must be obtained. The agreement will include the consent of the operator to install in a production drill hole modified casing furnished by the contract and permission to use the well for materials testing, monitoring, and probe testing for approximately two years.

The geothermal operator may a commercial venture or an experimental private or institutional operator under contract to a government agency, such as the national test site at East Mesa in the Imperial Valley of California. A cooperative agreement penmitting the modified casing installation might be arranged in principle by LBL, with installation and cost details to be negotiated between the prime contractor for Project 3 and the geothermal operator, subject to approval by LBL. To allow for negotiating flexibility, it might be well to have more than one candidate geothermal operator available.

\subsection{ANTICIPATED COSTS AND TIME FOR INSTRUMENT DEVELOPMENT}

It is expected that the development of a working subsidence monitoring system may cost about $\$ 625,000$, as follows:

$$
\begin{aligned}
& \text { PROJECT } 2 \text { - Design and Laboratory Test } \$ 150,000 \\
& \text { Wel } 1 \text { and Logging Components } \\
& \text { - Design and Laboratory Test 200,000 } \\
& \text { Probe Components } \\
& \text { - Management } \quad 60,000 \\
& \text { - Subtotal Project } 2 \quad \$ 410,000
\end{aligned}
$$




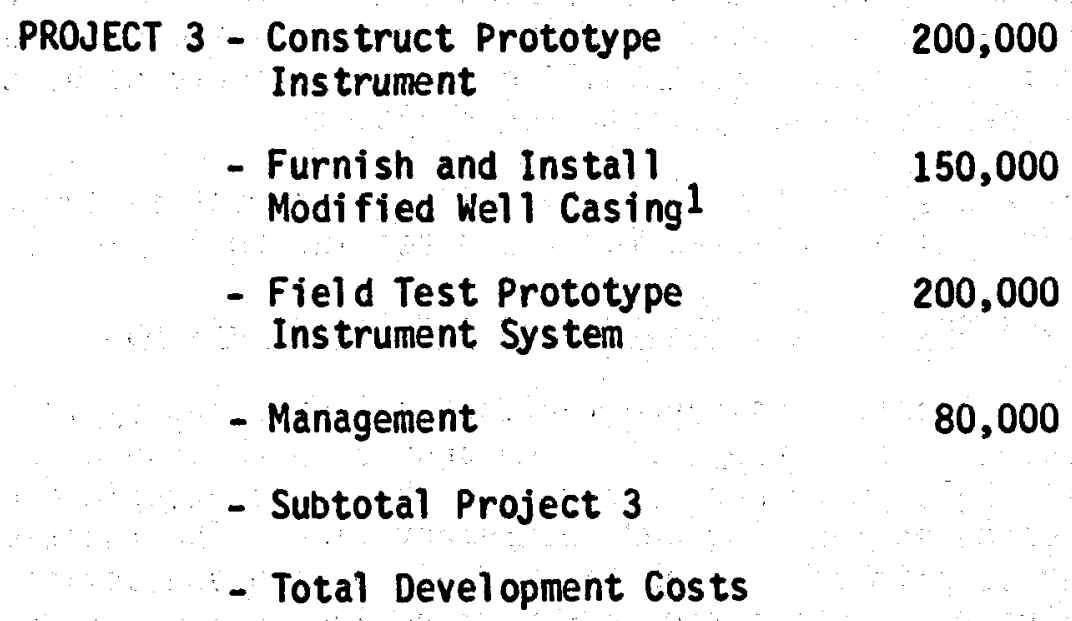

$\$ 1,040,000$

All costs presume that full advantage will be taken of all geothermal research efforts in related areas, so that the basic research for this project will be very specific and consequently not too costly.

Project 2 will take 1 year to 15 months to complete. Project 3 will take an additional 15 months to 2 years to complete. Its scheduling will depend on the availability of a geothermal well installation for field testing. These costs and schedule do not include verification of instrument ability to resist environmental damage and to monitor ground movements over a long-term monitoring program.

${ }^{1}$ Cost of modifications only 


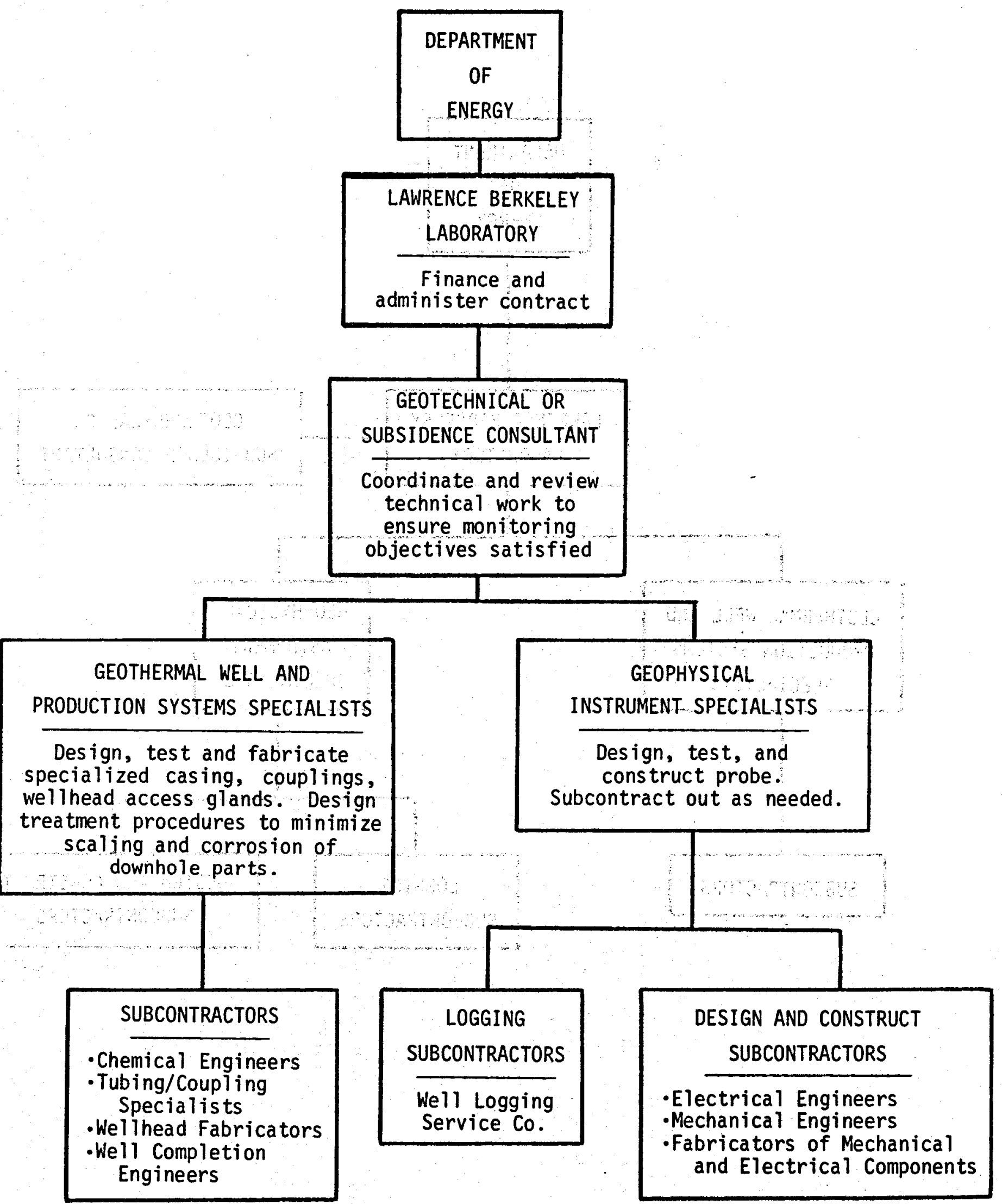

Figure 4.1 - RECOMMENDED MANAGEMENT STRUCTURE FOR SUBSIDENCE TOOL RESEARCH 


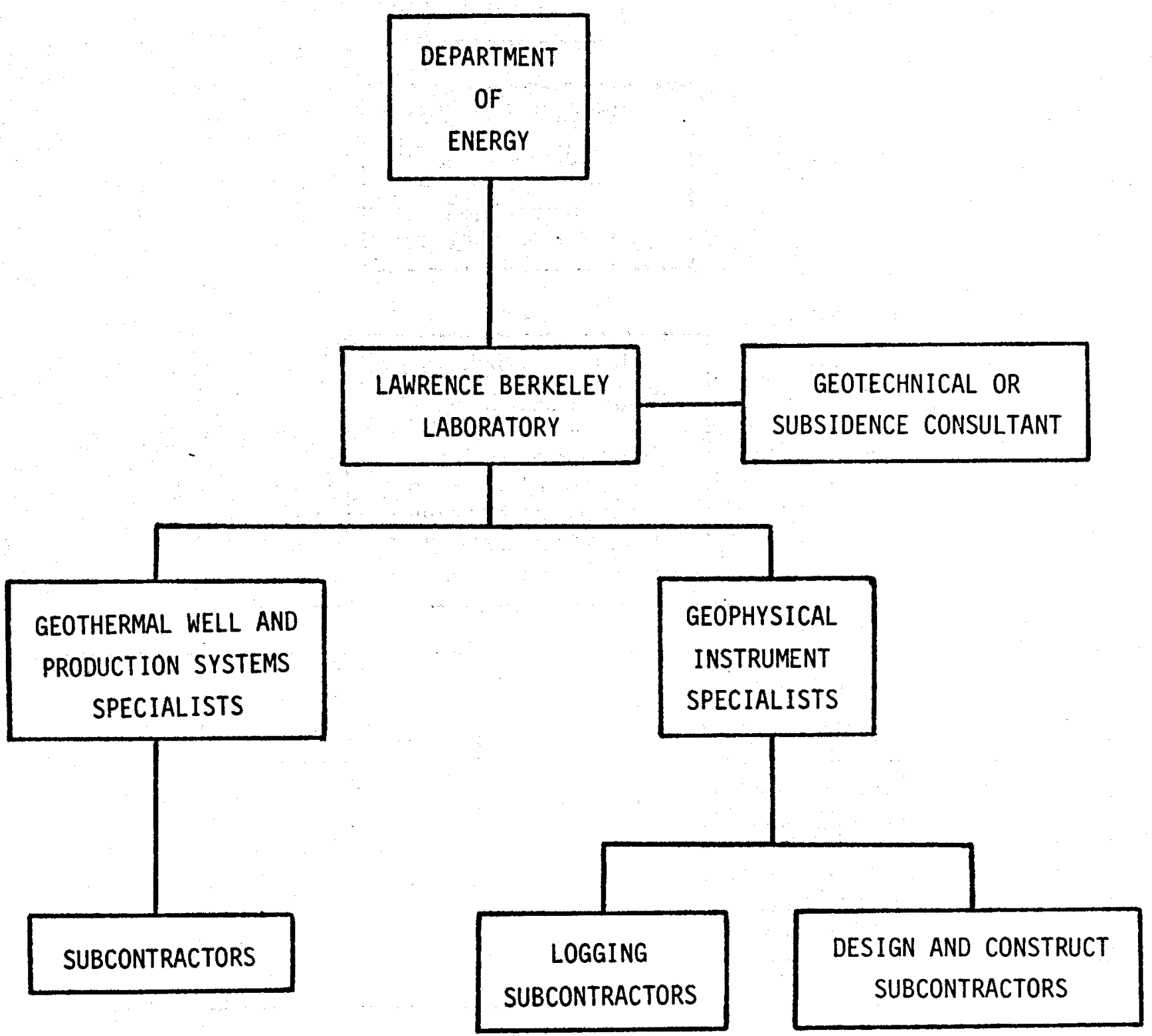

Figure 4.2 - ALTERNATE MANAGEMENT STRUCTURE FOR SUBSIDENCE TOOL RESEARCH 

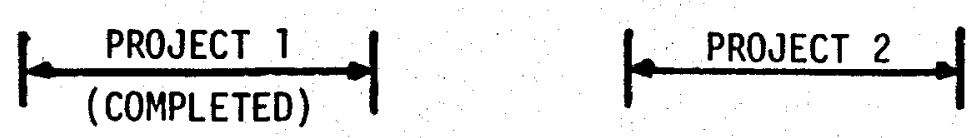

PROJECT 3

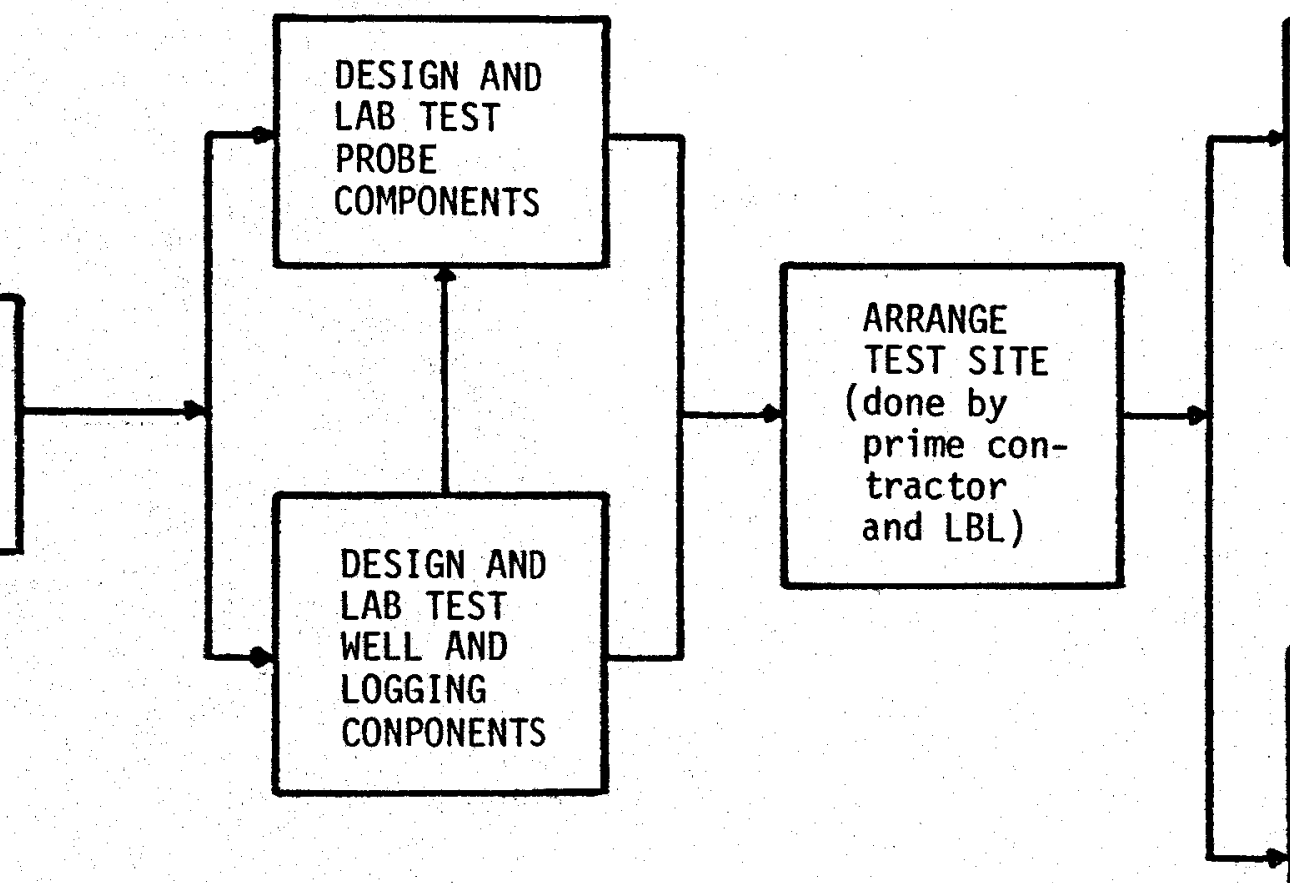

FURNISH AND

INSTALL

MODIFIED

WELL CASING

AND RELATED

EQU IPMENT

Figure 4.3 - OUTLINE OF PROPOSED GEOTHERMAL SUBSIDENCE RESEARCH - (CATEGORY 3) 
5.0 REFERENCES 


\section{REFERENCES}

Abrams, M. S., 1973, Compressive strength of concrete at temperatures to 1,6UUF: Portland Cement Association, Research and Development Bulletin RDO16, $11 \mathrm{p}$.

Ailtech 1975, Strain Gage Products: City of Industry, CA 91748.

Allegrini, G., and Benvenuti, G., 1970, Corrosion characteristics and geothermal power plant protection: UN Symposium on the Development and Utilization of Geothermal Resources, Pisa, in Geothermics, Special Issue 2, v. 2, pt. 1, p. $865-881$.

Allen, D. R., 1969, Collar and radioactive bullet logging for subsidence monitoring: SPWLA Tenth Annual Logging Symposium, P. G1-G19.

Allen, D. R., 1973, 0il field subsidence--susceptibility and monitoring techniques: presented to American Association of Petroleum Geologists Annual Meeting.

Allen, D. R., 1968, Physical changes of reservoir properties caused by subsidence and repressuring operations: Journal of Petroleum Technology, January, 1968, p. 23-29.

Allen, D. R., 1971, Update on collar and radioactive bullet logging for subsidence monitoring: unpublished, City of Long Beach, 5 .

Allen, D. R., 1977, Personal communication: Department of 0il Properties, City of Long Beach, California.

Altseimer, J. H., 1975, Geothermal well technology and potential application of subterrene devices - a status review: Second U.N. Symposium on the Development and Use of Geothermal Resources, Proceedings, San Francisco, v. 2, p. 1453-147u.

Aluminum Association Inc., 1976, Aluminum standards and data - 1976: the Aluminum Association, New York, 212 p.

American Society for Metals, 1977, Metal Progress 1977 Databook: Metal Progress, v. 112, no. 1, Mid-June 1977, 186 p.

Anon, 1978, Weigand Effect: A New Pulse - Generating Option: Automotive Engineering, February, p. 44-48.

Anon, 1977, Weigand Pulses break through into new applications: Canadian Controls and Instrumentation, December, p. 16-19.

ASHRAE, 1966, ASHRAE guide and handbook - fundamentals and equipment: American Society of Heating, Refrigerating and Air-Conditioning Engineers, New York, p. 1-28. 
Atherton, R. W., Finnemore; E. J., Gillam, M. L., DeGance, A. E., Nystrom, G. A., and Schainker, R. B., 1976. The analysis of subsidence associated with geothermal development: Volume I-Handbook, and Volume II-Research Report: Systems Control, Inc., Palo Alto, California, Reports 5139-1 and 5139-2.

BLH Electronics, 1977, Brochures: Waltham; MA 02154.

Babor, J. A., and Lehrman, A., 1954, Introductory College Chemistry: Thomas Y. Crowell Co., New York, $814 \mathrm{p}$ :

Bacon, C. F., 1976, Blowout of a geothermal well: California Geology, v. 29, no. 1, P. $13-17$.

Bajley, E. E., 1974, Strain gauges--what they are and their uses: Chartered Mechanical Engineer, v. 21, no. 8, p. 1U3-107.

Baker, L..E., Baker, R. P., Hughen, R. L., 1975, Report of the geophysical measurement in geothermal weils work shop - September 1975: Sandia Laboratories, AIbuquerque, N.M., SAND 75-U6U8, $69 \mathrm{p}$.

Baker, L. E., Campdeli, A. B., and Hughen, R. L., 1975a, Geothermal well logging - an assessment of the state of the art: The Log Analyst, v. 16, n. 6 , p. $21-24$.

Baker, L. E., Campbel1, A. B., Hughen, R. L., 1975b, We11-logging technology and geothermal applications - a survey and assessment with recommendations: Sandia Laboratories, Albuquerque, N.M., SAND 75-0275, 75 p.

Barnes, H. L., and Rimstidt, J. D., 1976, Chemistry of silica scale formation: in Second Workshop on Materials Problems. Associated with the Development of Geothermal Energy Systems, El Centro, California, May 1975, Bureau of Mines Open File Report 128-76, p. 1-14.

Barron, R., 1966, Cyrogenic systems: McGraw-Hill Book Co., New York, 678 p.

Batzle, M. L., and Simmons, G., 1976, Microfractures in rocks from two geothermal areas: Earth and Planetary Science Letters, v. 30, p. 71-93.

Bel teky, L., 1975, Problems related to operating geothermal wells subject to scaling in Hungary: Geothermics, v. 4, no. 1-4, p. 57-65.

Bjerrum, $L_{.,}$Johannessen, I. J., and Eide, $0 ., 1969$, Reduction of Negative Skin Friction on Steel Piles to Rock: Seventh International Conference on Soil Mechanics and Foundation Engineering, Proceedings, v. 2, p. 27-34.

Bleakley, W. B., 1973, Here's how Shell solves Uinta basin prodlems: The 0il and Gas Journal: Feb. 5, p. 45-5u.

Boyie, W. S., 1977, Light wave communications: Scientific American, v. 237 , no. 2 , p. $40-48$.

Bradley, F. N., 1971, Materials for magnetic functions: Hayden Book Co., Inc., New York, 348 p. 
Bromwell, L. G., Ryan, C. R., and Toth, W. E., 1971, Recording inclinometer for measuring soil movement: 4th Pan American Conference, San Juan, v. 2, p. 333-343.

Brookhaven National Laboratory, 1977, Alternate Materials for Construction for Geothermal Applications: Progress Reports 12 and 13, for DGEERDA, BNL 50699 and BNL 50665.

Brown, B. F., 1975, Environmentally assisted fracturing--research and standards: ASTM Standardization News, May, 1975, p. 8-16.

Budd, C. F., Jr., 1973, Steam production at the Geysers geothermal field, in Kruger, K., and Otte, C. (eds.), Geothermal energy: -- Resources, Production, Stimulation: Stanford University Press, Stanford, California, p. 129-144.

Bull, H. B., and Miller, R. E., 1975, Land subsidence due to ground-water wi thdrawal in the Los Banos-Kettlemen City area, California, part 1, changes in the hydrologic environment conducive to subsidence: U.S.G.S. Professional Paper 437-E, $71 \mathrm{p}$.

Burland, J. B., and Moore, J. F. A., 1973, The Measurement of Ground Displacement around Deep Excavations: Field Instrumentation in Geotechnical Engineering, Brihsh Geotechnical Society, Prooceedings, John Wiley \& Sons, New York, p. 70-84.

Burland, J. B., Moore, J. F. A., and Smith, P. D. K., 1972, A simple and precise borehole extensometer: Geotechnique, v. 22, no. 1, p. 174-177.

Burleigh Instruments, Inc., 1979, Super Invar Data Sheet and Personal Communication: Burleigh Park, Fishers, N.Y.

Burns, R. M., and Bradley, W. W., 1955, Protective Coatings for Metals: Reinhold Publishing Corp., New York, 643 p.

Carlson Instruments, 1978, Brochures: Campbel1, CA 95008.

Carpenter Steel Division, 1978, Brochure - Carpenter controlled - expansion alloys: Carpenter Technology Corporation, Reading, $\mathrm{Pa}$.

Case, L. C., 1970, Water Problems in 0il Production -- An Operator's Manual: Petroleum Publishing Co., p. 23-36.

Chemical Rubber Co. (CRC), 1966, Handbook of chemistry and physics: The Chemical Rubber Co., Cleveland, Ohio, 47 th ed.

Chen, Y. K., 1975, Chemistry, fate, and removal of trace contaminants from low and medium salinity geothermal waters: Presented to meeting of principle investigators of geothermal projects funded by National Science Foundation, held at Systems Control, Inc., Palo Alto, Calif.

Combs, J. B., 1975, The geology and geophysics of geothermal energy: Technology Review, v. 46. 
Cook, J. S., 1973, Communication by optical fiber: Scientific American, v. 229, Nov., p. 28-35.

Cording, E. J., et al., 1975, Methods for geotechnical observations and instrumentation in tunneling: Department of Civil Engineering, University of Illinois, Urbana, NSF Grant GI-33644X, UILU-ENG 75-2022, 2v.

Corrosion Control Handbook, 1970, Corrosion control methods: in Corrosion Control Handbook, Petroleum Engineer Publishing Co., p. 146-151.

Covington, L. C., 1978, Personal communication: Timet Division, Titanium Metals Corp. of America, Henderson, Nevada.

Covington, L. C., 1977, Titanium solves corrosion prodems in petroleum processing: Metai Progress, February, 1977, p. 38-45.

Cromling, J., 1973, Geothermal drilling in California: Journal of Petroleum Technology, AIME, September, 1973.

deLoos, J. M., 1973, In situ compaction measurements in Gronigen observation welis--the analysis of surface subsidence resulting from gas production in the Gronigen area, the Netherlands: Verhandelingen van het Koninklijk Nederlands geologisch mijnbouwkundig Genootschap, Royal Dutch Society of Geologists and Mining Engineers, Transactions, the Netherlanas, p. 79-104.

Dench, N. D., 1973, Well Measurements in Geothermal Energy: UNESCO, Earth Sciences 12, p. 85-96.

Denison, E. B., 1977, Shell's high-data-rate drilling telemetry system passes first test: The 0il and Gas Journal, June 13, p. 63-66.

Dodd, F. J., Johnson, A. E., and Ham, W. C., 1975, Material and corrosion testing at the Geysers geothermal power plant: Zna UN Symposium on the Development and Use of Geothermal Resources, San Francisco, v. 3, p. 1959-1963.

Donham, J. G., 1976, Chemical control of drillpipe corrosion: Materials Performance, v. 15, no. 10, p. 34-38.

Dunnicliff, C. J., 1971, Equipment for field deformation measurements: 4th Pan American Conference on Soil Mech. ana Foundation Engineering, Proceedings, San Juan, v. 2, p. 319-332.

Dunnicliff, C. J., 1970, Lecture notes, equipment and methods for measurement of displacements and settlements: Field Observations in Foundation Design and Construction, Seminar, Met. Section ASCE, Foundation and Soil Mechanics Groups, $31 \mathrm{p}$.

Echlin Mfg. Co., 1978, Brochures: Branford, CT, 06405.

Edwards, J.M., and Stroud, S.G., 1964, Field Results of the Electromagnetic Casing Inspection Log: Journal of Petroleum Technology, April, pp. 377-382. 
Electron Energy Corp., 1978, Brochures and Personnel Communication: Lanaisville, PA., July.

Ellis, A. J., 1975, Geothermal systems and power development, American Scientist, v. 63 , no. 5 , p. $509-521$.

Engineering, 1975, Thermal insulation: Engineering, v. 215, n. 1, p. I-VIII.

Evans, V., 1966, Plastics as corrosion-resistant materials: Pergamon Press, New York, 223 p.

Everard, N. J., and Tanner, J. L., 1966, Reinforced concrete design, Schaum's outl ine series: McGraw-Hili Book Co., New York, 325 p.

F-R Electronics Ltd., 1973, F-R Reed Switch Catalog, Wimborne, Dorset, U.K.

Fair, G. M., Geyer, J. C., and Okun, D. A., 1968, Water and Wastewater Engineering: John Wiley \& Sons, New York, vol. 2, p. 37:12-13.

Finnemore, E. J., and Gillam, M. L., 1976, Compaction processes and mathematical models of 1 and subsidence in geothermal areas: Proceedings, Second International Symposium on Land Subsidence, December 13-17, 1976, Anaheim, California, in press.

Fisher, E. N., 1978, Personal communication: E.N. Fisher \& Assocites, Belle Center, Ohio.

Fulcher, M. K., 1977, personal communication: U.S. Department of the Interior, Bureau of Reclamation, Boulder City, Nevada, Nov. 22, 1977.

Geonor AS, 1968, Brochures: 0s10 7, Norway.

Geospace, 1977, Brochures: Houston, TX 77036.

Giles, A.F., 1966, Electronic Sensing Devices: G. Newnes Ltd., London, U.K., 158 p.

Gilette, L. 0., 1975, Measurement of static strain at 20000F: Experimental Mechanics, v. 15, no. 8, p. 316-322.

Gl asstone, S., 1950, Sourcebook on Atomic Energy: D. Van Nostrand Co., Inc., New York, p. 136-142.

Glotzl, F., 1978, Brochures: Karlsruhe, W. Germany.

Goldsmith, P. R., and Hughes, J. M. 0., 1977, A Dorehole displacement gauge: Geotechnique, v. 27 , no. 2, p. 245-252.

Gould, J. P., and Dunnicliff, C. J., 1971, Accuracy of field deformation measurements: 4th Pan American Conference on Soil Mech. and Foundation Engineering, San Juan, v. 1, p. 313-366. 
Griffith, J. M., 1978, Mechanical seals in severe service face stiff requirements: $0 i 1$ and Gas Journal, v. $76, n .4$, p. 43-48.

Grindley, G. W., 1970, Subsurface structures and relation to steam production in the Broadiands geothermal field, New Zealand: Geothermics Special Issue 2, v. 2 , no. 1 .

Grindley, G. W., 1964, Geology of New Zealand geothermal steam fields, in Proceedings of the United Nations Conference on New Sources of Énergy, v. 2, no. 2, p. 237-245.

Gupta, R. C., Marshall, R. G., and Badke, D., 1973, Instrumentation for dykes on permafrost, Kettie generating station: Canadian Geotechnical Journal, v. 10, p. $410-427$.

Hanck, J. A., and Nekoksa, G., 1975, Corrosion rate monitoring at the Geysers geothermal power plant: Second UN Symposium on the Development and Use of Geothermal Resources, Proceedings, San Francisco, p. 1979-1984.

Harrison, M. R., and Pelanne, C. M., 1977, Cost-effective thermal insulation: Chemical Éngineering, December 19, p. 62-76.

Harting, D. R., 1976, Evaluation of a capacitive strain measuring system for use to 15U0of: ISA Transactions, v. 15, no. 1, p. 8-16.

Hartley, R. A., 1972, Coatings and corrosion: Journal of Materials, v. 7, no. 3, p. 361-379.

Hatley, H. M., 1971, Pipeline corrosion -- the legal aspects: Anti-Corrosion, November, p. 6-7.

Heikes, R. R., and Ure, R. W. Jr., 1961, Themoelectricity - Science and Engineering: Interscience Publishers, New York, $576 \mathrm{p}$.

Hermannsson, S., 1970, Corrosion of metals and the forming of a protective coating on the inside of pipes carrying thermal waters used by the Reykjavik Municipal District Heating Service: UN Symposiun on the Development and Utilization of Geothermal Resources, Proceedings, Pisa, in Geothermics Special Is sue 2, v. 2, pt. 2, p. 16U2-1612.

Hewlett-Packard (HP), 1964, Time Domain Reflectometry: Hewlett-Packard Co., Application Note 62, Palo Al to, CA.

Hewlett-Packard Co. (HP), 1965, Cable testing with time domain reflectometry: Hewlett-Packard Co. Application Note 67, Colorado Springs, C0, 18 p.

Hirasuna, A.R., Bilyeu, G.D., Davis, D.L., Stephens, C.A., and Veal, G.R., 1977, Geothermal Elastometric Materials: Tweive-Months Progress Report submitted by L'Garde, Inc., Newport Beach, CA, to DGE-ERDA, 84 pp. 
Hirono, T., 1969, Niigata ground subsidence and ground water change in Land Subsidence: Proceedings of the Tokyo Symposium, IAHS/AIHS/UNESCO, v. 1, P. 144-161.

Hoffman, M. R., 1975, Brine chemistry -- scaling and corrosion: Environmental Quality Laboratory, California Institute of Technology, Pasadena, California, EQL Memorandum No. 14, 48 p.

House, P. A., Johnson, P. M., and Jowse, D. F., 1975, Potential power generation and gas production from Gulf Coast geopressure reservoirs: California University, Lawrence Livermore Laboratory, Report 51813, $40 \mathrm{p}$.

Howell, R. C., Wright, F. D., and Dearinger, J. A., 1976, Ground movement and pressure changes associated with shortwall mining: Site Characterization, 17 th U.S. Symposium on Rock Nechanics, Proceedings, 6 p.

Hunt, T. M., 1970, Net mass loss from the Wairakei geothermal field, New Zeal and: UN Symposium on the Development and Utilization of Geothermal Resources, Proceedings, Pisa, in Geothermics Special Issue 2, v. 2, pt. 1, p. 487-4y1.

INCO, 1949, Iron nickel and related alloys of the invar and elinvar types - data sheet A: The International Nickel Co. Inc., New York, 20 p.

INC0, 1963, Mechanical and physical properties of the austenitic chronium-nickel stainiess steels at elevated temperatures: The International Nickel Co. Inc., New York, $17 \mathrm{p}$.

INC0, 1968, Properties of some metals and alloys: The International Nickel Co. Inc., New York, $63 \mathrm{p}$.

Joffe, A. F., 1958, The revival of themoelectricity: Scientific American, v. 199, n. 5, p. $31-37$.

John Chatillon \& Sons, 1978, Brochures - Isoelastic spring alloy: John Chatilion \& Sons, Kew Gardens, New York.

Johnson, C. S. 1976a, Telemetry data systems-- Part 1--Frequency Division Multiplexing: Instrumentation Technology, v. 23 , no. 8, p. 3y-43.

Johnson, C. S., 1976b, Telemetry data systems--Part II--Time Division Multiplexing: Instrumentation Technology, v. 23, no. 10, p. 47-53.

Johnston Division, 1972, Ground water and wells: Johnston Division, Universal 0 il Products, St. Paul, Minn., 440 p.

Kallstenius, T., and Bergau, W., 1961, In situ determination of horizontal ground movements: 5 th International Conference on Soil Mech. and Foundation Engineering, v. 1, p. 481-485.

Kirklen, C. A., 1974, Effectiveness of well casing cathodic protection -- an analysis: Journal of Petroleum Technology, July, p. 724-73u. 
Koenig, J. B., 1969, The Geysers geothermal field: California Division of Mines and Geology Mineral Information Service, v. 22, no. 3, p.-123-128.

Kokesh, F. P., 1951, Gamma-Ray Logging: 0il and Gas Journal, Vol. 50, No. 12, July 26, p. $284-300$.

Kolker, M., Greene, A.H., Kasevich, R.S., Robertson, J.C., and Grossi, M.D., 1978, Raytheon Downhole Information System-Electromagnetic Borehole Measurements while Drilling System: Final Report, Prepared for D.0.E. Department of Geothenmal Energy by Raytheon Company, Advanced Development Laboratory, Wayland, MA, $140 \mathrm{pp}$.

Komaki, S. 1969, On the variation of artesian head and land-surface subsidence due to groundwater withdrawl, in Land Subsidence: Proceedings of the Tokyo Symposium, IAHS/AIHS/UNESCO, v. 1, p. 256-271.

Kovach, R. L., 1974, Source mechanisms for Wilmington oil field, California subsidence earthquakes: Seismological Society of America bulletin, v. 64, no. 3 , p. 699-711.

Kryukov, P. A., and Larionov, E. G., 1970, Physico-chemical sampling of high temperature wells in connection with their encrustation by calcium carbonate: UN Symposium on the Development and Utilization of Geotherinal Resources, Proceedings, Pisa, in Geothermics, Special Issue 2, v. 2, pt 2, p. 1624-1628.

Lambe, T. W., and Whitman, R. V., 1969, Soil mechanics: John Wiley \& Sons, Inc., New York, $553 \mathrm{p}$.

LaMori, P. N., 1975, Hydrothermal geothermal resources and growth in utilization: Electric Power Research Institute, Palo Al to, California, in preparation.

Lee, K. L., and Shen, C. K., 1969, Horizontal movements related to subsidence: Journal of the Soil Mechanics and Foundation Division, ASCE, v. 95, no. SM1, p. 139-166.

Lofgren, B. E., 1961, Measurement of compaction of aquifer sy'stems in areas of 1 and subsidence: USGS Professional Paper 424-B, P. B49-B52.

Lofgren, B. E., 1968, Analysis of stresses causing land subsidence: U.S. Geological Survey Professional Paper 600-B, P. B219-B225.

Lofgren, B. E., 1969, Field measurement of aquifer-system compaction, San Joaquin Valley, California, USA, in Land Subsidence: Proceedings, IAHS/AIHS/UNESCO, Tokyo, p. 272-284.

Lofgren, B. E., 1973, Monitoring ground movement in geothermal areas: Hydraulic Engineering and the Environment, Proceedings, Hydraulics Division, ASCE, p. $437-447$.

Lofgren, B. E., 1974, Measuring ground movement in geothermal areas of Imperial Valley, California: Conference on Research for the Development of Geothermal Energy Resources, Jet Propulsion Laboratories, Pasadena, Calif., p. 128-138. 
Lofgren, B.E., 1978, Personal Communication: Woodward-Clyde Consultants, San Francisco, CA.

Lula, R. A., 1976, Ferritic stainless steels -- corrosion resistance and economy: Metal Progress, July, D. 24-29.

Lynes, Inc., 1979, 1978-1979 Catalog: Houston, TX 77017.

McConmick, J.B., ed., 1978, High-Temperature Electronics Workshop: Geothermal Energy Magazine, Vo1. 6, No. 9, Sept., pp. 11-14.

McDonald, W. J., 1978a, MWD looks best for direcional work and drilling efficiency: MWD-State of the Art-1, The 011 and Gas Journal, Mar. 27, p. 141-147.

McDonald, W. J., 1978b, Four different systems used for MWD: MWD-State of the Art-2, The 0il and Gas Journal, April 3, p. 115-124.

MCDonald, W. J., and Ward, C. E., 1975, Borehole telemetry is key to continuous downhole drilling measurements: The $0 i 1$ and Gas Journal, Sept. 15, p. 111147.

McLellan, H. J., 1965, El ements of physical oceanography: Pergamon Press, New York, p. 117-124.

McQueen, D. A., 1974, Junk car anodes may cut cathodic protection costs: World 0 il, v. 178 , no. 4 , p. 71 .

Maihak, H., 1977, Brochures: Hamburg, 60, W. Germany. Malloy, J. F., 1969, Thermal insulation: Van Nostrand Reinhold Co., New York,

Marsland, A., and Quarterman, R., 1974, Further development of mul tipoint magnetic extensometers for use in highly compressible ground:

Geotechnique, v. 24, no. 3, p. 429-433.

Martin, C. A., and Rust, D. H., 1976, Hostile environment logging: The Log Analyst, v. 17, n. 2, p. 3-1U.

Martin, R. E., 1975, Guide to better insulation: Chemical Engineering, May 12, p. $98-100$.

Matsuo, K., 1973, Drilling for geothermal steam and hot water in Geothermal Énergy: Unesco, Earth Sciences 12, p. 73-83.

Maurer, J. R., 1975, New austentitic and ferretic stainless steels for geothermal applications: 2nd workshop on materials problems associated with the development of Geothermal Energy, Proceedings, BuMines OFR 128-76, p. 1U5-119. 
Mayuga, M. N., 1970, Geology and development of California's giant-Wilmington oil field, in Geology of Giant Petroleum Fields: American Association of Petrology and Geology, Memoir 14, p. 158-184.

Mayuga, M. N., and Allen, D. R., 1969, Subsidence in the Wilmington oil field, Long Beach, California, U.S.A., in Land Subsidence: Proceedings, IAHS/AIHS/UNESCO, TOKYo, p. 66-79.

Meidav, H. T., and Sanyal, S., 1976, A comparison of hydrothermal reservoirs of the western United States: Electric Power Research Institute, Palo Alto, California, EPRI ER-364 Topical Report 3, 170 p.

Melcor, 1978, Brochures and personal communication: Materials Electronic Products Corporation, Trenton, N.J.

Metal Progress, 1975, Superalloys for superservice: Metal Progress, August, p. 37-46.

Mitre Corporation, 1978, Prospects for Improvement in Geothermal Well Technology and Their Expected Benefits: prepared for Division of Geothermal Energy-D.0.E., No. HCP/T4014-04, 128 pp.

Moffitt, F. H., and Bouchard H., 1973, Surveying: Intext Educational Publishers,
New York, 879 p.

Moffitt, L. R., 1972, Time-domain reflectometry-theory and applications: Hewlett-Packard Application Note 75, Colorado Springs, CO, $14 \mathrm{p}$.

Morris, W. S., 1970, Fighting scale in salt water lines, in Corrosion Control Handbook: Petroleum Engineer Publishing Co., P. 5u-52.

Mueller, W.A., Kalfayan, S.H., Reilly, W.W. and Ingham, J.D., 1978, Development and Evaluation of Elastometric Materials for Geothermal Applications: Annual Report October 1976-0ctober 1977, Prepared for Department of Energy, Division of Geothermal Energy by Jet Propulsion Laboratory, California Institute of Technology, Pasadena, CA $54 \mathrm{pp}$.

Nair, V. V., 1977, Variable oscillator reacts to magnetic flux changes: Electronics, November 24, p. 130, Reprinted from Electronics, November 24, 1977; Copyright (C) McGraw-Hill, Inc., 1977 all rights reserved.

Neale, L., 1974, Potentiometers: Engineering materials and design, v. 18, no. 10, p. 32-34.

Needham, P. B., Jr., and staff, 1975, Materials research and development program for geothermal environments: 2nd workshop on materials problems associated wi th the development of Geothermal Energy, Proceedings, BuMines OFR 128-78, p. 45-62.

Nicholson, R. W., 1974, Charts find acceptable dog-leg severity limits: $0 i l$ and Gas Journa1, April 15, 1974, p. 72-82.

Noltingk, B. E., 1974, Measuring static strains at high temperature: Experimental Mechanics, v. 14, no. 10, p. 420-423. 
$0 i 1$ and Gas Journal, 1971, Directional survey instrument gets field tests: The $0 i 1$ and Gas Journal, April 5, 1971, p. 66-67.

O'Rourke, J.E., 1977, U.S. Patent No. 4003263: Moraga, California, January, 18.

Ostroff, A. G., 1970a, Understanding and controlling oilfield corrosion, in Corrosion Control Handbook: Petroleum Engineer Publishing Co., p. 2-14.

Ostroff, A. G., 197Ub, Understanding and controlling oilfield corrosion, in Corrosion Control Handbook: Petroleum Engineer Publishing Co., p. $1 \overline{5-2 U}$.

Ozawa, T. and Fujii, Y., 1970, A phenomenon of scaling in production wells and the geothermal power plant in the Matsukawa area: UN Symposium on the Development and Utilization of Geothermal Resources, Pisa, in Geothermics Special Issue 2, v. 2, pt. 2, p. 1613-1618.

Pahler, R.H., Jr., and Roberts, A.S., Jr., 1977, Design of a Fiber Uptic Pressure Transducer: Trans. ASME, Feb. 1977, pp. 274-280.

Palmer, D. W., 1977, Hybrid microcircuitry for 3000C operation: IEEE Transactions non Parts, Hybrids and Packaging, $v$. PHP-13, no. 3, p. 252257.

Palmer, D.W., 1978, Hybrid Microcircuitry for $300^{\circ} \mathrm{C}$ Operation: Geothermal Energy Magazine, Vol. 6., No. 9, Sept., Pp. 24-29.

Palmer, D. W., Draper, B. L., McBrayer, J. D., and White, K. R., 1978, Achve devices for high temperature microcircuitry: Sandia Laboratories SAND77$1145,120 \mathrm{p}$.

Palmer, D.W. and Gayard, F.P.; 1978, Aluminum Wire to Thick-Film Connections for High-Temperature Operation: IEEE Transactions on Components, Hybrids, and Manufacturing Technology, Vol. CHMT-1, No. 3, Sept., pp. 219-222.

Palmer, D.W. and Heckman, R.C., 1978, Extreme Temperature Range Microelectronics: IEEE Transactions on Components, Hybrids, and Manufacturing Technology, Vol. CHMT-1, No. 4. Dec., pp. 333-340.

Palmer, J. D., 1976, Specialists check on metal oddities: Canadian Chemical Processing, February, p. 30-34.

Palmer, T. D., 1975, Characteristics of geothermal wells located in the Salton Sea geothermal field, Imperial County, California: Lawrence Livermore Laboratory, Livermore, Calif., UCRL-51976, 54 p.

Papadopulos, S. S., Wallace, R. H., Jr., Wesselman; J. B., and Taylor, R. E., 1975, As sessment of onshore geopressured-geothermal resources in the northern Gulf of Mexico basin, in White, D. E., and Williams, D. L. (eds.), Assessment of geothermal resources of the United States--1975: U.S. Geological Survey, Circular 726, p. 125-146.

Peterson, F. L., and Davis, S. N., 1969, Short-term movement of the land surface near water wells: Land Subsidence, Proceedings, IAHS/AIHS/UNESCO, Tokyo, p. 360-367. 
Phillips, J. N., and Sykes, C. R., 1970, Temperature, pressure--key hurdles ahead in ultradeep wells: 0 il and Gas Journal, July 20, 1970, p. 65-70.

Poland, J. F., 1976, Land subsidence stopped by artesian-head recovery, Santa Clara Valley, California in Second International Symposium on Land Subsidence: Proceedings, Anaheim, California, p. 124-132.

Poland, J. F., 1978a, Personal Communication in Review of WCC Interim Report No. 1: Sacramento, CA., February

Poland, J.F., 19780, Personal Communication: U.S.G.S.-W.R.D., Sacramento, CA, october.

Poland, J.F., and Davis, G. H., 1969, Land subsidence due to withdrawal of fluids: Reviews in Engineering Geology, Geological Society of America, v. 2, p. 187-270.

Poland, J. F., and Ireland, R. L., 1965, Shortening and protrusion of a well casing due to compaction of sediments in a subsiding area in California: U.S. Geological Survey Professional Paper 525-B, p. 180-183.

Portland Cement Association (PCA), 1968, Effect of Various Substance on Concrete and Protective Treatments, Where Required: Portland Cement Association, Skokie, Illinois, General Information Pamphlet No. 3, 11 p.

Potts, E. L., 1964, Current investigations in rock mechanics and strata control: 4th International Conference on Strata Control and Rock Mechanics, H. Krumb School of Mines, Columbia University, New York City, P. 29-45.

Powel 1, J.A., 1974, A Simple Two-Fiber Optical Displacement Sensor: Rev. Sci. Instrum., Vol. 45, No. 2, copyright 1974 by American Institute of Physics.

Ramey, H. J., Jr., 1968, A reservoir engineering study of the Geysers geothermal field: submitted as evidence, Reich and Reich, Petitions versus Commissioner of Internal Revenue, 1969 Tax Court of the United States, 52 T.C., no. 74,76 p.

Rehm, W.A., 1979, Drilling and Producing Wet Steam Wells at Cerro Prieto, Mexico: Petroleum Engineer International, No. 5, Yol. 51, Apr.,
Pp. 78-90.

Renner, J. L., White, D. E., and Williams, D. L., 1975, Hydrothermal convection systems, in White, D. E., and Williams, D. L. (eds.), Assessment of Geotherma TResources of the U.S.--1975: U.S. Geological Survey Circular 726 , p. 5-57.

Rex, R. W., and Howel1, D. J., 1973, As sessment of U.S. geothermal resources, in Kruger, $P_{.}$, and Otte, E. (eds,), Geothermal Energy, Resources Production, Stimulation: Stanford University Press, Stanford, Calif., p. 59-67. 
Riley, F. S., 1969, Analysis of borehole extensometer data from central

California: Land Subsidence Proceedings, IAHS/AIHS/UNESCO, Tokyo, p. 424-431.

Riley, F. S., 1978, Personal communication: U.S.G.S., Denver, Co.

Rosato, D. V., 1968, Other properties and characteristics: in Rosato, D. V., and Schwartz, R. T. (eds.), Environmental Effects on Polymeric Materials, Interscience Publishers, New York, p. 733-1102.

Runyan, E. E., 1965, An engineered approach to cement-lined tubing: Journal of Petroleum Technology, June, 1965, p. 626-632.

Sandia Laboratories, 1978a, GeoEnergy Technology: Sandia Laboratories, Albuquerque, N.M., June, 16 pp.

Sandia Laboratories, 1978b, Geothermal Logging Instrumentation Development Progress Report: Sandia Laboratories, Albuquerque, N.M., Dec., $29 \mathrm{pp}$.

Sandia Laboratories, 1979, Lab News: Alduquerque, N.M., Vol. 31, No. 7, Apr. 6.

Sano, S., 1969, Observation of compaction of formation in the land subsidence of Ni igata City: Land Subsidence, Proceedings, IAHS/AIHS/UNESCO, Tokyo, p. 401-409.

Schrier, L. L., (ed), 1976, Corrosion: George Newnes, London, 2 vols.

Selig, E. T., and Grangaard, O. H., Jr., 1970, A new technique for soil strain measurement: Materials Research and Standards, v. 1U, no. 10, p. 19-36.

Sellers, J. B., 1969, Rock mechanics instrumentation for salt mining: Third Symposium on Salt, N. Ohio Geological Society, v. 2, p. 236-248.

Shaevitz Engineering, 1972, Handbook of Measurement and Control: by E.E. Herceg, HB-72, Pennsauken, N.J.

Sharpe, W. N. Jr., 1975, Strain gages for long-term high-temperature strain measurement: Experimental Mechanics, v. 15, n. 12, p. 482-488.

Silberberg, J. H., 1976, Reservoir fluid sampling and analysis for a geopressured geothermal we11: Second Geopressured Geothermal Energy Conference, Proceedings, v. 3, p. 57-82.

Slope Indicator Co. (SINCO), 1977, Brochures: Seattle, WA 98103.

Smith, P. D. K., and Burland, J. B., 1976, Performance of a high precision multipoint borehole extensometer in soft rock: Canadian Geotechnical Journal, v. 13, p. $172-176$.

Snape, E., and Van Royden, D., 1969, Sweet-well corrosion of sucker-rod steels: The 0 il and Gas Journal, June 30, 1969, p. 98-105. 
Snyder, R.E., 1978, Geothermal Well Completions: An Overview of Existing Methods in Four Types of Developments: Prepared by Completion Technology Co., Houston, TX, SAND 78-7010, 128 pp.

Speller, F. N., 1951, Corrosion, causes and prevention: McGraw-Hill Book Co., Inc., New York, $686 \mathrm{p}$.

Spinnler, R. F., and Stone, F. A., 1978, MWD program nearing commerciality: MWD State of the Art-4, The 0il and Gas Journal, May 1, p. 59-66.

Standard Wire and Cable Co., 1977, Brochure: Standard Wire and Cable Co., El Segundo, California.

Starr, D., 1978, Personal communication: W.B. Driver Co., New Jersey.

Stengel, R. F., 1977, Pulse Generator produces rate-independent vol tage: Design News, April 18, 2 p.

Stilwell, W. B., Hall, W. K., and Tawhai, J., 1975, Ground movement in New Zealand geothermal areas: 2nd U.N. Symposium on the Development and Use of Geothermal Resources, Proceedings, San Francisco, California.

Teledyne, 1971, Thermoelectrics in Teledyne Report - 1st Quarter 1971: Teledyne, Inc., Los Angeles, Ca., p. I-10.

Teledyne, 1978, Brochures -- telan-2: Teledyne Energy Systems, Timonium, Maryiand.

Telemac International Inc., 1970, Brochures: Paris, France.

Terzaghi, K., and Peck, R. B., 1967, Soil mechanics in engineering practice: John Wiley \& Sons, Inc., New York, 729 p.

Thomas, H. P. and Luscher, U., 1978, Improvement of Bearing Capacity of Pipe Piles by Corrugations: unpublished paper prepared for Joing US-USSR Seminar on Building Under Cold Climates and Permafrost, Leningrad, USSR, October, $8 \mathrm{p}$.

Thorhallson, S., Ragnars, K., Arnorsson, S., and Kristmanndottir, H., 1975, Rapid scaling of silica in two district heating systems: 2nd $U . N$. Symposium on the Development and Use of Geothermal Resources, Proceedings, San Francisco, p. 1445-1449.

Toliva, E., 1970, Corrosion measurements in a geothermal environment: U.N. Symposium on the Development and Utilization of Geothermal Resources, Pisa, in Geothermics Special Issue 2, v. 2, pt. 2, p. 1596-1601.

Tomlinson, M. J., 1969, Foundation design and construction: Pitman Publishing, London, $785 \mathrm{p}$.

Traynor, B. $V_{0}, 1978$, Electrodril demonstration program shows promise: MWD-State of the Art-3, The 0il and Gas Journal, April 17, p. 108-129. 
Tskhvirashvilli, P., Vardigoreli, O., and Alcosin, P., 1972, On corrosion of metals in geothermal power plants: Geothermics, v. 1, no. 3, p. 113-118.

Turner, W. C., 1974, Criteria for installing insulation systems in petrochemical plants: Chemical Engineering Progress, v. 70, n. 8, p. 41-45.

Uhlig, H. H. (ed.), 1948, The corrosion nandbook: John Wiley Sons, New York, $1188 \mathrm{p}$.

U.S. Bureau of Reclamation (USBR), 1966, Concrete Manual: U.S. Government Printing Of fice, Washington, 7 th ed., $642 \mathrm{p}$.

U.S. Department of Interior, 1976, Geothermal resources operational orders issued under the Geothermal Steam Act of 1970: U.S. Department of the Interior, Geological Survey, Conservation Division, Office of the Area Geothermal Supervisor, GRO Order Nos. 1-4.

Van der Knapp, and van der Vi is, A. C., 1967, On the cause of subsidence in oil producing areas: 7 th World Petroleum Congress 3, Mexico City Proceedings, Elsevier Scientific Publishing Company, The Hague, p. 85-95.

Van Vlack, L. H., 1964, Elements of materials science: Addison-Wesley Publishing Co., Inc., Reading, Mass., 445 p.

Varnado, S.G. and Colp, J.L., 1978, Report of the Workshop on Magma/Hydrothemal Drilling and Instrumentation: Sandia Laboratories, Albuquerque, N.M., 68 pp.

Veneruso, A.F., Polito, J., and Heckman, R.C., 1978, Geothermal Logging Instrumentation Development Program Plan: Prepared by Sandia Laboratories, Albuquerque, N.M., for U.S.-E.R.D.A., SAND 78-0316, Aug., $72 \mathrm{pp}$.

Veneruso, A.F., 1979, Personal Communication: Sandia Laboratories, Albuquerque, Apr.

Veneruso, A.F., and Stoller, H.M., 1978, High Temperature Instrumentation for Geothermal Applications: Geothermal Resources Council, Transactions, Vol. 2, July, pp. 679-682.

Waddell, G. G., 1964, Application of instrumentation in determining rock behavior during stoping at the Star Mine, Burke, Iadho: Proceedings, Sixth Symposium on Rock Mechanics, University of Missouri, Rolla, p. 23-42.

Wahl, E., and Yen, I., 1975, Scale deposition and control research for geothermal utilization, in Second U.N. Symposium on the Development and Use of Geothermal Resources: Proceedings, San Francisco, v. 3, p. 1855-1864.

Wahl, E. F., 1976, Scale deposition in geothermal brines, in Second Workshop on Materials Problems Associated with the Development of Geothermal Energy Systems: El Centro, California, May 1975, U.S. Bureau of Mines Open File Report 128-76, p. 15-44.

Walker, G. M. 1977, Weigand Effect Getting Practical: Electronics, April 28, p. 85-86. 
Weeter, R. F., and Chandler, R. J., 1974, Mutual interference between well casings with cathodic protection: Materials Performance, v. 13, no. 1 , p. 26-30.

West, E. R., 1977, Snowbank drilling in the Delaware basin of west Texas: SPEAIME Deep Drilling and Product. Symposium, Amarillo, Texas.

White, D. E., and Williams, D. L. (eds.), 1975, Assessment of geothermal resources of the United States--1975: U.S. Geological Survey, Circular $726,155 \mathrm{p}$.

White, D. E., 1973, Characteristics of geothermal resources, in Geothermal Energy: Resources, Production, Stimulation, edited Paul Kruger and Carel 0tte, with the permission of the publishers, Stanford University Press. (C) 1973 by the Board of Trustees of the Leland Stanford Junior University.

White, D. E., 1974, Diverse origins of hydrothermal ore fluids: Economic Geology, v. 69, p. 954-973.

Whiting, R. L., 1973, Possible effects of geothermal water and steam production on the subsurface environment: Proceedings, 74th National Meeting of AICHE, New Orleans, Paper No. 50B, p. 762-771.

Wilson, J. S., Shepherd, B. P., and Kaufman, S., 1974, An analysis of the potential use of geothermal energy for power generation along the Texas gulf coast: Dow Chemical U.S.A., Texas Division, 63 p.

Wilson, S. D., 1962, The use of slope measuring devices to determine movements in earth masses, in Field Testing of Solls: ASTM Special Technical

Publication No. 322, p. 187-198.

Wolfe, R., 1964, Magnetothermoelectricity: Scientific American, June, p. 70-81.

Wonn, J.W., 1978, Acoustic Sensor Development for Geothemal Borehole Televiewer: Geothermal Resources Counc1l, Transactions, Vo1. 2, July, Pp. 735-738.

Woodward-Clyde Consultants (WCC), 1977a, Available subsurface instrumentation for geothermal subsidence: Unpublishea report submitted to Lawrence Berkeley Laboratory, Berkeley, by Woodward-Clyde Consultants, San Francisco, Interim Report Ho. 1, $40 \mathrm{p}$.

Woodward-Clyde Consultants, (WCC), 1977b, Subsidence monitoring systems for undermined areas: unpublished report submitted to the U.S. Bureau of Mines, Denver, by Woodward-Clyde Consultants, San Francisco, CA, 304 p.

Woodward-Clyde Consultants (WCC), 1978a, Methods for Improving Available Subsidence Instrumentation: Unpublished report submitted to Lawrence Berkeley Laboratory, Berkeley, by Woodward-Clyde Consultants, San Francisco, Interim Report No. 2, 40 p. 
Woodward-Clyde Consultants (WCC), 1978b, Sensor and Signal Technology for Geothermal Subsidence Monitoring: Unpublished report submitted to Lawrence Berkeley Laboratory, Berkeley, by Woodward-Clyde Consultants, San Francisco, Interim Report No. 3, 34 pp.

Woodward-Clyde Consultants (WCC), 1978c, New Concepts in Subsidence Instrumentation: Unpublished report submitted to Lawrence Berkeley Laboratory, Berkeley, by Woodward-Clyde Consultants, San Francisco, Interim Report No. 4, 15 pp.

Yanagase, T., Suginohara, Y., and Yanagase, K., 197U, The properties of scales and methods to prevent them: UN Sumposium on the Development and Utilization of Geothermal Resources, Proceedings, Pisa, in Geothermics Special Issue 2, v. 2, pt. 2, p. 1619-1623.

Yeakley, L. M., and Lindholm, U.S., 1974, Development of capacitance strain transducers for high-temperature and biaxial applications: Experimental Mechanics, v. 14, no. 8, p. 331-336.

Yerkes, R. F., and Castle, R. 0., 1969, Surface information associated with oil and gas field operations in the United States, in Land Subsidence: Proceedings of the Tokyo Symposium, IASH/AISH/UNESCO, v. 1, p. 55-56.

Zapffe, C. A., 1949, The corrosion resistance of the stainless steels, Stainless Steels, American Soceity for Hetals, p. 60. 
APPENDIX A

REPORT UPDATE IN

RESPONSE TO

GEOTHERMAL SUBSIDENCE

WORKSHOP 


\section{TABLE OF CONTENTS}

LIST OF TABLES AND FIGURES $\frac{\text { Page }}{i \mathbf{i}}$

1.0 INTRODUCTION

2.0 MONITORING SYSTEM COMPONENTS

2.1 Component Description 1

2.2 Influence of System Components on System Performance 2

3.0 MEASUREMENT REQUIREMENTS 2

3.1 Probable Users/Uses of Data 3

3.2 Specifications 3

4.0 RE-EVALUATION OF SLIP COUPLINGS 4

4.1 Past Use of Slip Couplings for Subsidence Monitoring 4

4.2 Compatibility of Slip Couplings with the Geothermal
Environment

4.3 Compatibility of Slip Couplings with Existing We11
Completion Techniques

4.4 Conclusion 9

$\begin{array}{lll}5.0 & \text { DEPTH PROBLEMS } & 10\end{array}$

5.1 Probes with Three or More Sensors 10

$\begin{array}{ll}5.2 \text { Strain-Free Inserts } & 10\end{array}$

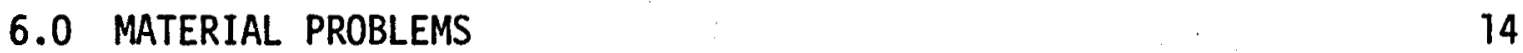

6.1 Super-Invar 14

6.2 High Temperature Electronics and Logging Tools 14

$\begin{array}{ll}7.0 \text { REFERENCES } & 19\end{array}$ 


\section{LIST OF TABLES AND FIGURES}

$\underline{\text { Page }}$

TABLE 6.1 - SUPER-INVAR DATA SHEET 15

TABLE 6.2 - GEOTHERMAL LOGGING TOOLS UNDER DEVELOPMENT 18

TABLE 6.3 - HIGH TEMPERATURE ELASTOMER AND SEALS 20

Figure 4.1 - COMPARISON OF RIGID AND SLIP COUPLINGS 5

Figure 4.2 - CASING ADJUSTER FOR WELLS IN SUBSIDING AREAS 7

Figure 5.1 - CORRECTION FOR PROBE ACCELERATION 11

Figure 5.2 - POSSIBLE APPLICATION OF STRAIN-FREE INSERT IN PRODUCTION WELL

Figure 6.1 - EXPANSION CURVES FOR LOW EXPANSION ALLOYS 16 
APPENDIX A: REPORT UPDATE IN RESPONSE TO GEOTHERMAL SUBSIDENCE WORKSHOP

\subsection{INTROOUCTION}

In October 1978, a Geothenmal Subsidence Workshop was held on the Geothermal Subsidence Research Program, sponsored by D,O.E. and managed by Lawrence Berkeley Laboratory. At that workshop, Woodward-Clyde presented the results of this study, LBL directed that the feedback from the workshop be incorporated into our report. This appendix contains our summaries of relevant opinions expressed at the workshop by industry representatives, government regulators, and reservoir engineers and modelers. It al so contains the results of some additional research performed to answer some of the questions raised at the workshop. The workshop provided constructive criticisms and suggestions which we believe have contributed significantly to the value of this report.

This appendix details selected problems raised at the workshop. In the following sections, we discuss:

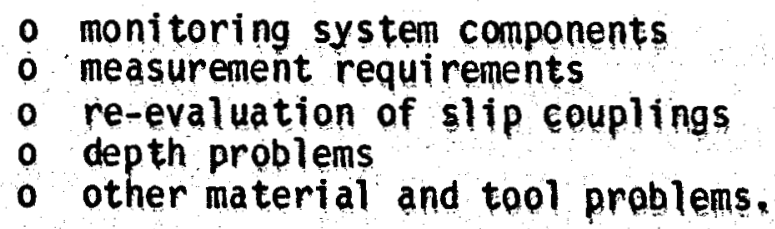

These discussions are included as an Appendix to allow the main text to remain a concise summary of our research. Pertinent points fran this appendix are incorporated in the main text.

\subsection{MONITORING SYSTEM COMPONENTS}

Same participants at the Geothemal Subsidence Workshop were not familiar with the various components of a subsurface monitoring system to detect ground displacements. Since the perfomance of a monitoring system depends on the capabilities of each of its components, we priefiy review here the elements which comprise inclinometer and extensometer systems.

\subsection{Component Description}

COMPONENT

Borehole

Markers

\section{DESCRIPTION}

In geothemal practice, a variety of hole completion techniques are used including unlined sections, slotted liners, and cemented, cased holes.

Markers are used to identify points in the fomation to be studied. Two examples are grouted anchors and radioactive bullets shot into the formation. For inclinometer use, the markers may be simply marks on the logging cable indicating distance from the downhole ins trument to the surface cable reel. 
Sensing Device (with readout)

Logging System

Orientation Device
In an extensometer, the sensing device locates the markers and determines the relative vertical displacement between them. In an inclinometer, the sensing device measures the relative angular rotation at the points under study; these points are located by the length of cable played out.

Most extensometer and inclinometer systems use sensing devices which must be lowered down a borehole to make readings. A logging system may include a winch, electrical cable, access gland and other equipment for handling the sensing device.

Inclinometers only. An orientation device such as a gyroscope or grooves in a casing is needed to indicate the compass bearing of the measured angular movement.

\subsection{Influence of System Components on System Performance}

While every engineer can identify some aspect of an instrumentation system which he feels is most critical, it is important to recognize that each component of an instrumentation system will influence its performance. In particular, the quality of the markers and sensing device should be comparable. An example from surveying may clarify this point. Good practice prohibits the use of low accuracy wooden rods or wooden stakes with a high accuracy first order level. Rather, an invar rod and carefully constructed benchmarks are used. Similarly then for subsurface monitoring, markers which provide a fuzzy signal are not compatible with precision sensing devices. Conversely, if the economics of geothermal well completion prohibits installation of precision markers in the well, then it may be sensible to develop a subsurface subsidence monitoring tool that provides only approximate measurement of downhole compaction.

In the main text of the report, we discuss ways in which each of the components of a compaction monitoring system can be improved to provide better performance. However, we recognize that, in the final analysis, the components for a compaction monitoring system for geothermal use will be selected based not only on the desired perfomance level but al so on economic constraints.

\subsection{MEASUREMENT REQUIREMENTS}

In Interim Report 1 (Appendix B) and in Section 2.1.1 of this report, we briefly examined what accuracies and other monitoring characteristics might be useful to people studying subsidence phenomena. However, a detailed study and interviews with potential data users was beyond the scope of this study. The workshop provided valuable insight into (1) who might use the data and why compaction data may be collected, and (2) what specifications might be desirable for instrument development. The discussion below summarizes some issues and opinions expressed at the workshop. 


\subsection{Probable Users/Uses of Data}

Two motivating factors will probably be responsible for subsurface compaction instrument development and use: (1) expectation of future federal regulation, and (2) data requirements for reservoir engineering. It was felt that specific federal requirements for subsurface subsidence monitoring were not expected, and subsurface monitoring would only be used if specifically required. Industry representatives appeared hesistant to endorse development of a compaction monitoring tool because they feared it might encourage federal regulation along those lines. With respect to (2), the modelers and reservoir engineers felt that in-situ compaction measurements were valuable because they (a) would permit a direct comparison of laboratory and field measurements of rock response, (b) they would help refine reservoir models, and $(c)$ they would be useful to help minimize reservoir damage. However, one attendee commented that, at Wilmington, subsurface compaction data from the field measurements were not used to any extent by reservoir engineers. Many of the workshop attendees envisaged compaction monitoring as a long-range scientific tool, not necessarily required by the DOE "On-line" date of 1985.

\subsection{Specifications}

3.2.1 Magnitude of Movement and Associated Accuracy - Because of brine disposal problems, injection will almost certainly be practiced. In most geothermal areas, brine will be returned to the producing reservoir. As a result of injection, the magnitude of subsidence movements is likely to be small, although it may vary from the injection area to the withdrawal area. Consequently, to observe compaction where reinjection is practiced, sensitive instruments will be required to detect and define the compacting zones. Thus, the specifications detailed in Table 2.8 (in main text) are reasonable and possibly even too lax.

In geopressured areas (Snyder, 1978), the situation may be somewhat different. Early laboratory data suggest that reinjection may substantially reduce the permeability in the fine sands of the geopressured zones (Snyder, 1978). Also very high injection pressures will be required to match downhole pressures, overcome flow loss of head, and ensure adequate fluid take. Consequently, waste brine may be reinjected into relatively shallow non-producing zones (Snyder, 1978). Without this source of recharge to the producing reservoir, the large movements (5-7 im total compaction) listed in Table 2.8 are more likely to develop. "Thus, geopressured wel is may be monitored satisfactorily with coarser instrumentation than is needed for other geothermal areas.

\subsubsection{Areal Extent of Monitoring}

At the Geothermal Subsidence Workshop, the question of the areal extent of monitoring was raised. A study of the number of installations in a given field and the locations of those installations was beyond the scope of this project. However, this point merits further study, since the number of required installations will affect the relative costs and benefits of each of the candidate instrument systems. 


\subsection{RE-EVALUATION OF SLIP COUPLINGS}

At the Geothermal Subsidence Workshop, industry representatives were resistant to concepts that required modification of the well to improve instrument system accuracy (such as the use of slip couplings). Their hesistancy is understandable since increased installation costs and more complicated technical problems would result from such modifications.

In our first Draft Final Report (October, 1978) we discussed several techniques for improving the accuracy of measurements, including the use of markers shot into the formation and the use of slip couplings. These modifications were proposed (1) to minimize the influence of the steel and cement in the borehole on the measured subsidence, and (2) to provide markers that accurately track formation movements. A convenient way to monitor ground movements is to monitor casing deformation and assume it corresponds to formation compaction. We were concerned that with this technique, rigid couplings might hinder accurate definition of the zone of compaction, as shown in Figure 4.1. The use of slip couplings can improve the sensitivity of techniques which monitor casing deformation as an indicator of formation compaction. Consequently, all four of the recommended instrument systems in the Draft Final Report incorporated slip couplings. In view of the comments at the workshop, we briefy re-examined some questions associated with the use of slip couplings, including:

- past use for subsidence monitoring

o compatibility with the geothermal environment

o compatibility with existing geothermal well completion techniques.

\subsection{Past Use of Slip Couplings For Subsidence Monitoring}

In this section, the previous use of slip couplings in subsidence ares is reviewed.

Couplings which allow slip have been used to prevent subsidence damage in oil and groundwater subsidence areas. Mayuga and Allen (1969) note that the use of a well completion technique that combined a compression joint and a bell hole packed with a viscous fluid was used to protect oil wells at Wilmington, California, against subsidence damage. Poland (1978b) notes that the Geological Survey installed slip joints in the San Joaquin and Santa Clara Valleys. In one well in western Fresno County, he observed, "We installed 8 slip joints in this well $2000 \mathrm{ft}$ deep in 1958. As of now we have measured 12.3 feet of compaction at this well. It is still functioning as a compaction recorder. I am sure it would have failed many years ago wi thout slip joints."

The USGS has generally designed and fabricated their own slip couplings (Poland, 1978b; Lofgren, 1978). As shown in Figure 2.8a of the main text, commercial casing expansion joints are available, although their design function is to absorb thermal length changes in production casing rather than subsidence-induced length changes. A commercial "Casing Adjuster" for subsidence damage control was available for a limited time from the Creighton 0 il Tool Co. and was used at Wilmington and at Lake Maracaibo, 


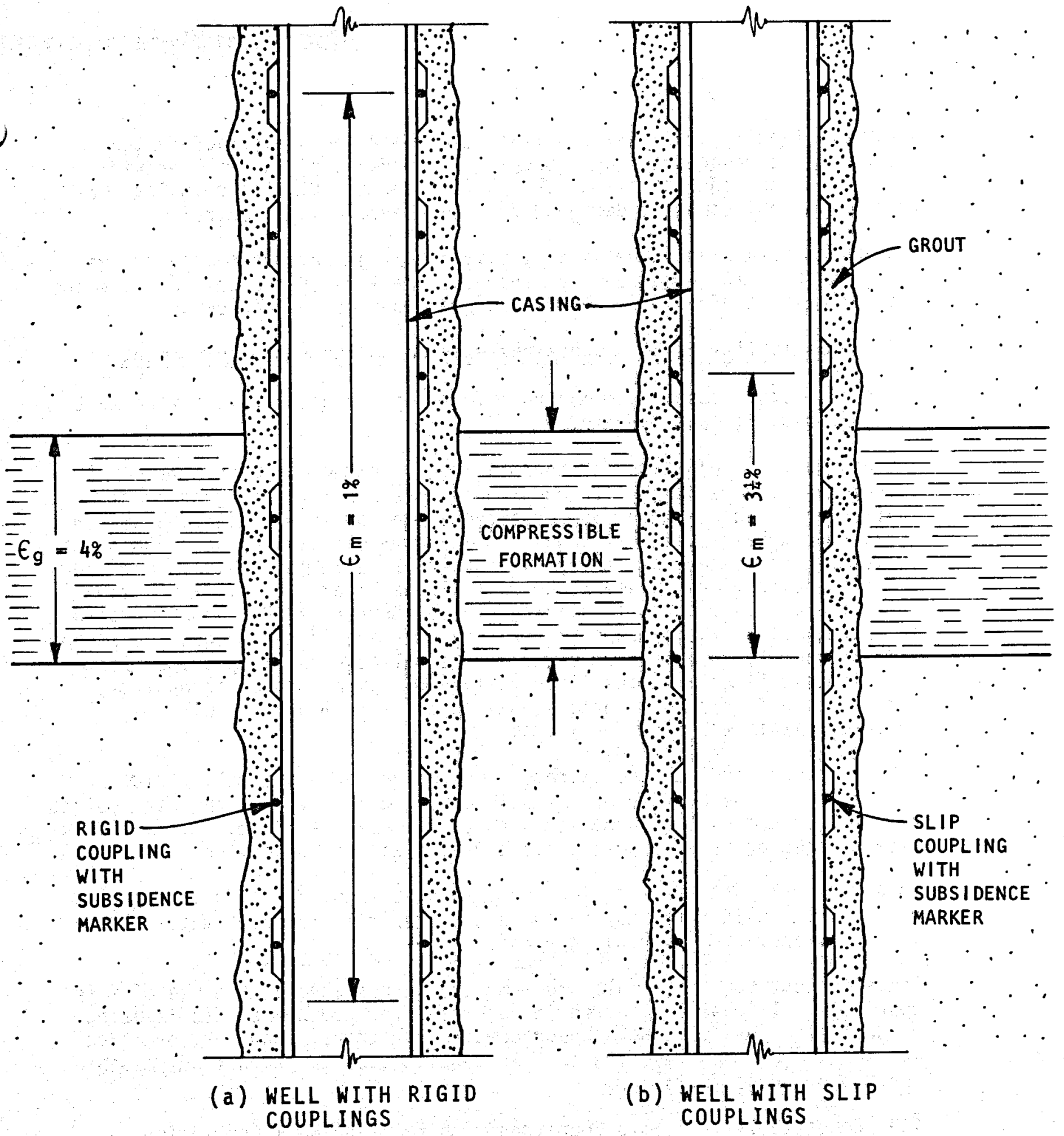

NOTES:

(1) $\epsilon_{m}=$ measured strain; $\epsilon_{g}=$ ground strain

(2) In well (a) with rigid couplings, some slip between the casing and the formation may occur making it difficult to define the zone undergoing compaction. In well (b), the zone of compaction is defined more precisely with slip couplings. 
Venezuela (see Figure 4.2). Currently, A and A Tools manufacture the Creighton casing adjuster and Bowen Tools of Houston, TX, will provide slip joints on request (Allen, 1979). of a commercial slip coupling currently available that is designed for subsidence damage control.

The above discussion suggests that slip couplings have been used successfully in subsiding areas and can increase the life of the well and improve the performance of instrumentation such as compaction recorders.

\subsection{Compatibility of Slip Couplings with the Geothermal Environment}

In this section, possible damaging effects of the geothermal environment on slip couplings are examined.

The problems of adjusters for thermal length change may be comparable to potential problems which slip couplings may encounter in a geothermal environment. In oil wells where string expansion is expected due to temperature changes after well completion, a downhole casing expansion joint is used in the innermost string (see Figure 2.8a in main text). For geothermal applications, however, no suitable seals are yet available to allow the use of a downhole sliding sleeve (Snyder, 1978). The approach in geothermal wells has been to fix the lower end of the production string and incorporate an expansion spool in the well lhead to accommodate thermally-induced length changes (Snyder, 1978). New elastomer technology, discussed in Section 6.0 , offers hope that the seal prodlem will be resolved within the next few years.

Other problems include the extremely corrosive, abrasive well fluid. Unless the inside profile of the slip coupling is very smooth, high points will be abraded by the formation sand carried by the fluid. Also at diameter changes or in recesses, scale products will be deposited.

An additional potential prodlem for slip couplings, as shown in Figure $2.8 \mathrm{~b}$, of the main text, may be the use of several different materials, which may encourage gaivanic corrosion.

It is evident that materials problems are an obstacle to the use of slip couplings. Intensive research in materials for geothermal use is being conducted now by DOE and $i$ ts geothermal contractors. However, practical application of this research to slip coupling design may not be possible for two or three years.

\subsection{Compatibility of Slip Couplings with Existing Well Completion Techniques}

The instrument systems developed in this project utilize slip couplings because they provide an accurate way to track subsurface movements. In this section, we assess whether this dependence on slip couplings is appropriate. First of all, the well must be cased or lined to use slip couplings. Secondly, slip couplings are effective as markers for monitoring only if there is a certain minimum adhesion between the casing and the formation. In that case, the compression of the casing (concentrated at 

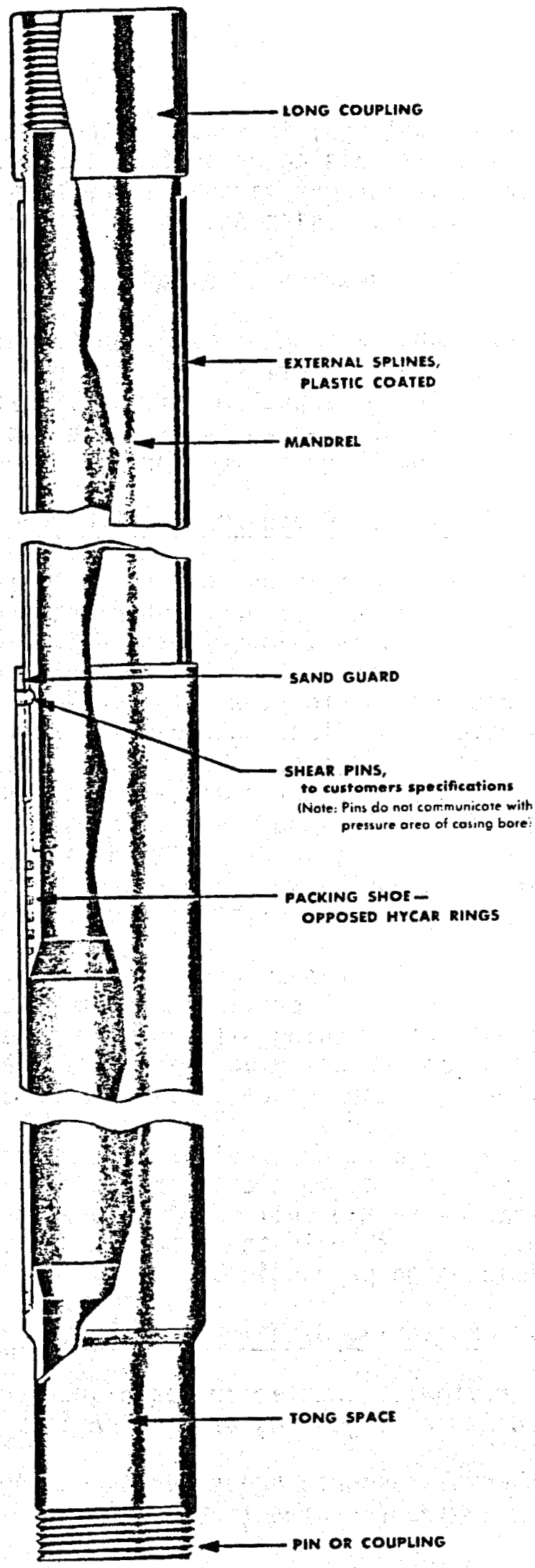

Figure 4.2 - CASING ADJUSTER FOR WELLS IN SUBSIDING AREA
(Creighton, 195 
the slip couplings) is equal to the compression of the formation over that interval. If this adhesion is not present, the couplings may not be as effective as markers since all the slip may concentrate at the coupling with the lowest resistance to sliding.

Thus, in this section we examine:

(1) What are the completion techniques?

(2) When are wells cased/lined?

(3) Can we expect minimum casing/formation adhesion?

(4) To what extent will we have to use methods other than slip couplings to monitor formation movements around a well?

\subsubsection{Review of Geothermal Wel 1 Completion Techniques}

In all geothermal areas, casing is fully cemented above the producing formation. Practice differs within the production zone. The casing program at the Geysers is unique in the U.S. Decause of the competent nature of the geologic formation. An open hole completion is used to provide minimum restrictions to flow. In the Imperial Valley, wells are lined in the production zone since downhole formations are not competent enough for open hole completions. Most of ten, uncemented slotted liners are used, a) though one operator does cement casing and perforate it. Significant skin effects and sand proauction may result with the use of slotted, uncemented liners. Snyder (1978) suggests that these problems will become apparent as more power plants are constructed, as more wells are drilled, and as longer-term operating data become available for existing wells.

Sand production can be minimized by several techniques (Snyder, 1978). One approach is to provide support to the formation around the well so that natural bridging will prevent sand movement. This approach also ensures good coupling between the formation and the casing for accurate monitoring. One method, gravel packs around slotted liners, is expected to gain acceptance in the Imperial Valley (Snyder, 1978). Cementing and perforating a liner provides even more support. This technique may receive some use in the Imperial Valley, but will probably gain greater acceptance in the Gulf Coast geopressured areas. Sands there are considerably finer than in the Imperial Valley and will require more support to minimize sand production.

\subsubsection{Potential Site for Slip Coupling Use}

This review of completion techniques answers several of the questions posed at the beginning of Section 4.3 .

Geothermal resource areas having casing or liners in which slip couplings could be incorporated include:

- all geothermal resource areas above the producng zone (especially if compaction due to other sources is occurring in these formations) 


\section{o al1 geopressured areas}

- all liquid-dominated wells.

The minimum casing/ground adhesion needed to make slip couplings act as accurate formation markers will be present:

0 in cemented wells

o in gravel packed, lined wells

o in wells with slotted liners, provided the formation is soft enough to move in and grip the liner, and provided erosion around the liner is limited.

Alternate techniques for accurate tracking of formation movements must be considered for:

- Vapor-dominated wells in the producing zone

- Some liquid-dominated wells with slotted liners in the producing zone.

\subsection{Conclusion}

The approach in this research project has been to try to use producing wells for subsidence monitoring. As a result we concluded, as presented in earlier reports, that slip couplings would improve the quality of the data. This conclusion was based on subsidence phenomena and measurement experience in non-geothermal areas. The review in this secion has indicated that while the slip coupling technique is valid, its potential for use may not be as extensive as we had hoped. Our conclusions on slip couplings are presented in the following paragraphs.

Casing movement in a cemented well may not be representative of formation movement without the use of slip couplings. Slip couplings can prevent casing damage and improve instrumentation performance. These conclusions were reached in earlier reports.

The use of slip couplings offers the most potential for geopressured wells, where the combination of potentially large movements (Section 3.0 ) and the use of cemented casing favor slip coupling use. Also, a longer development time is available to al low materials problems in slip coupling design to be overcome. Slip couplings also appear to be suitable for producing zones with gravel-packed slotted liners and for some wells using only slotted liners.

Slip couplings are compatible with well completion techniques in all geothemal wells above the production zone. They will improve the sensitivity of the measurement. 
In the producing zones of vapor and some liquid-dominated wells, it appears that alternate techniques for tracking formation displacements should be explored. Some techniques are discussed in the main text. They include:

- use of markers (such as radioactive or magnetic bullets) shot into the formation around producing wells

- use of auxiliary casing attached to production well casing

- use of a separate installation for compaction monitoring -- fully cemented in the producing zone and incorporating slip couplings or bellows sections.

of these, the use of markers shot into the formation will probably minimize the capital investment in permanent downhole subsidence monitoring equipment.

\subsection{DEPTH PROBLEMS}

One problem which is encountered by probe type monitoring devices is variable velocity of the probe caused by "tool bounce" and "cable stretch." This effect can be minimized by the use of three or more sensors per probe (Main Text --Section 2.2.5) or the use of strain-free sections in casing (Main Text -- Section 2.4.3). Some brief comments on these two techniques follow.

\subsection{Probes with Three or More Sensors}

Figure 2.4 in the main text shows how the distance between downhole markers can be calculated from the signals from a three sensor probe travelling at a constant velocity between the two markers. If the ratio of the distances $L_{12}$ and $L_{23}$ (Main Text -. Figure 2.4) as shown by the strip chart recorder is different from that on the probe, the velocity of the probe is probably not constant. If one makes the assumption that the acceleration of the probe is constant over the interval between the markers, then a slightly more accurate estimation of distance can be obtained, as shown on Figure 5.1 in this appendix. The error, if this correction is not made, is about eleven percent at a constant acceleration of $1 \mathrm{~m} / \mathrm{sec}^{2}$, reducing to one percent at $10^{-4} \mathrm{~m} / \mathrm{sec}^{2}$ acceleration. Typical logging rates and marker distances were used in the calculations.

\subsection{Strain-Free Inserts}

In Figures 2.13, 2.14, and 2.15 in the main text, we suggest the use of strain-free inserts for use in auxiliary casing. Strain-free inserts provide a known length downhole and were proposed to reduce the measurement problems caused by varying probe velocity as it traverses the borehole. At the Geothermal Workshop, D. Allen (City of Long Beach) suggested that this technique would also work in producing wells. A sketch of such a system is shown in Figure 5.2. Its advantage is that the cost of installation is less than for an auxiliary casing installation. Also, in common 


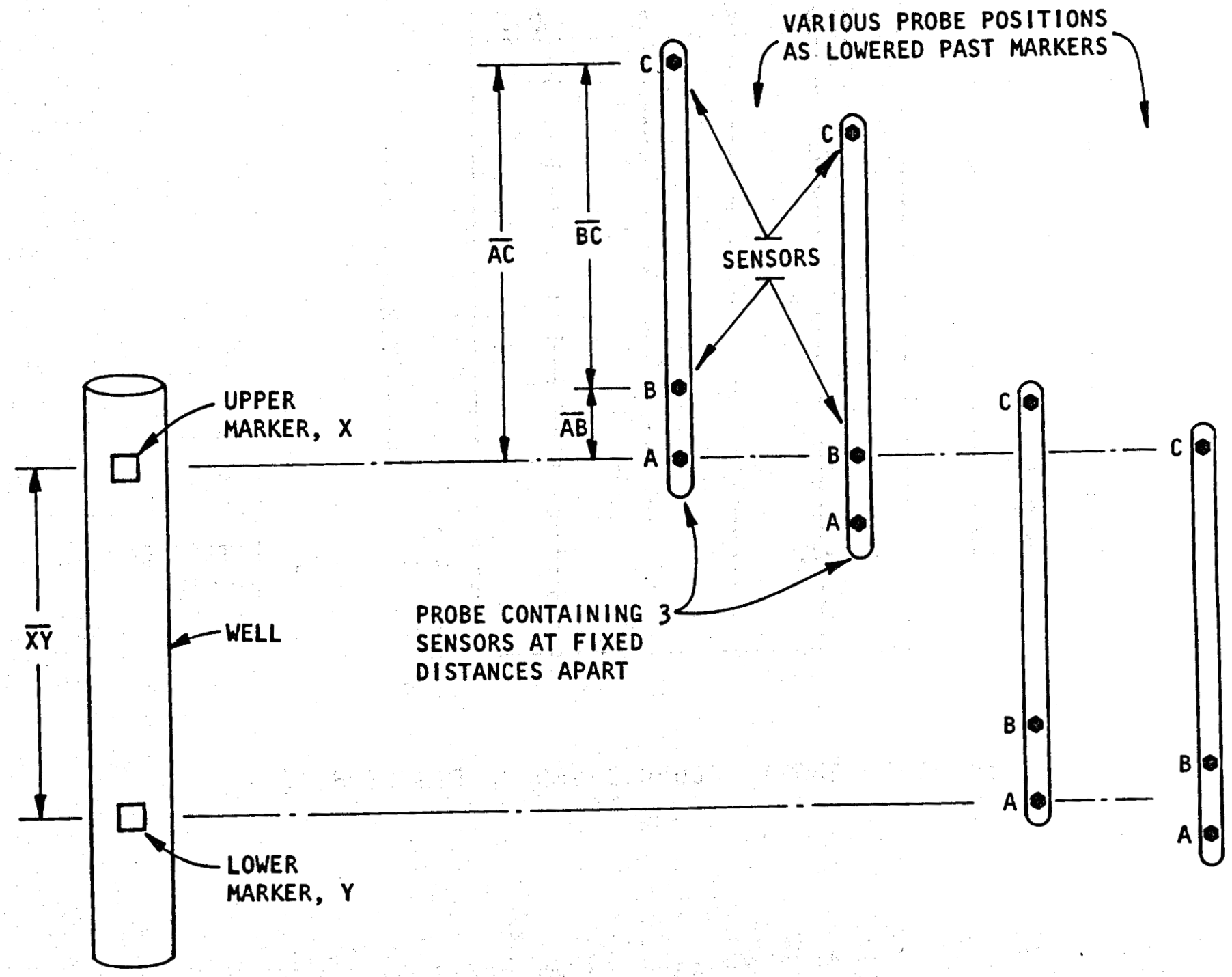

(a) POSITION OF PROBE AS EACH SENSOR RECORDS MARKER

Figure 5.1 - CORRECTION FOR PROBE ACCELERATION 


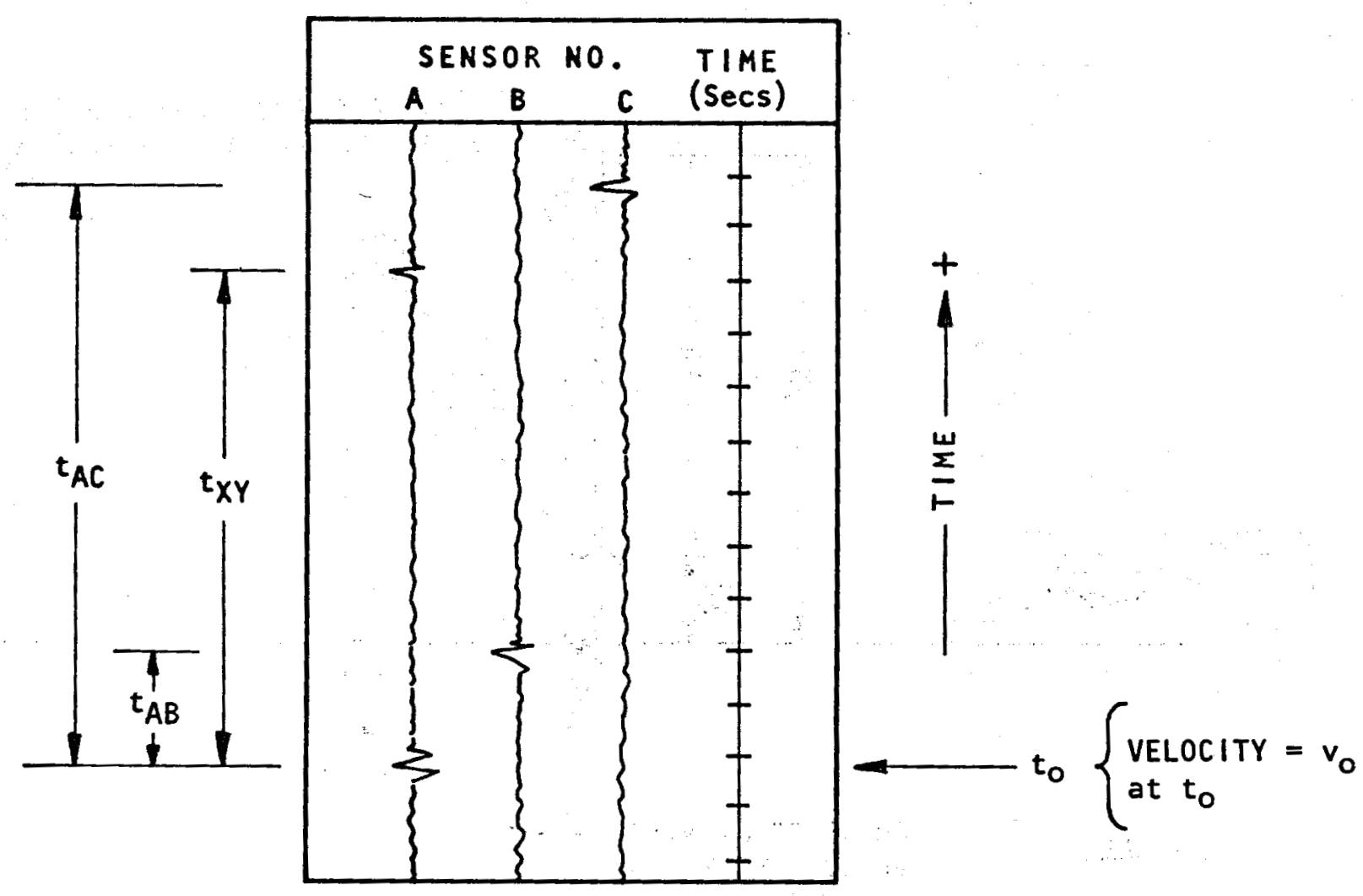

(b) STRIP CHART RECORD BASED ON FIGURE 5.1 (a)

$$
\left.\begin{array}{l}
\overline{A B}=v_{0} t_{A B}+\frac{1}{2} a t_{A B}^{2} \\
\overline{A C}=v_{0} t_{A C}+\frac{1}{2} a t_{A C}^{2}
\end{array}\right\} \text { Solve for a and } v_{0}
$$

then:

$\overline{X Y}=v_{0}\left(t_{X Y}\right)+\frac{1}{2} a\left(t_{X Y}\right)^{2}$

(c) CALCULATIONS TO DETERMINE SPACING BETWEEN MARKERS

Figure 5.1 (Cont'd) - CORRECTION FOR PROBE ACCELERATION 


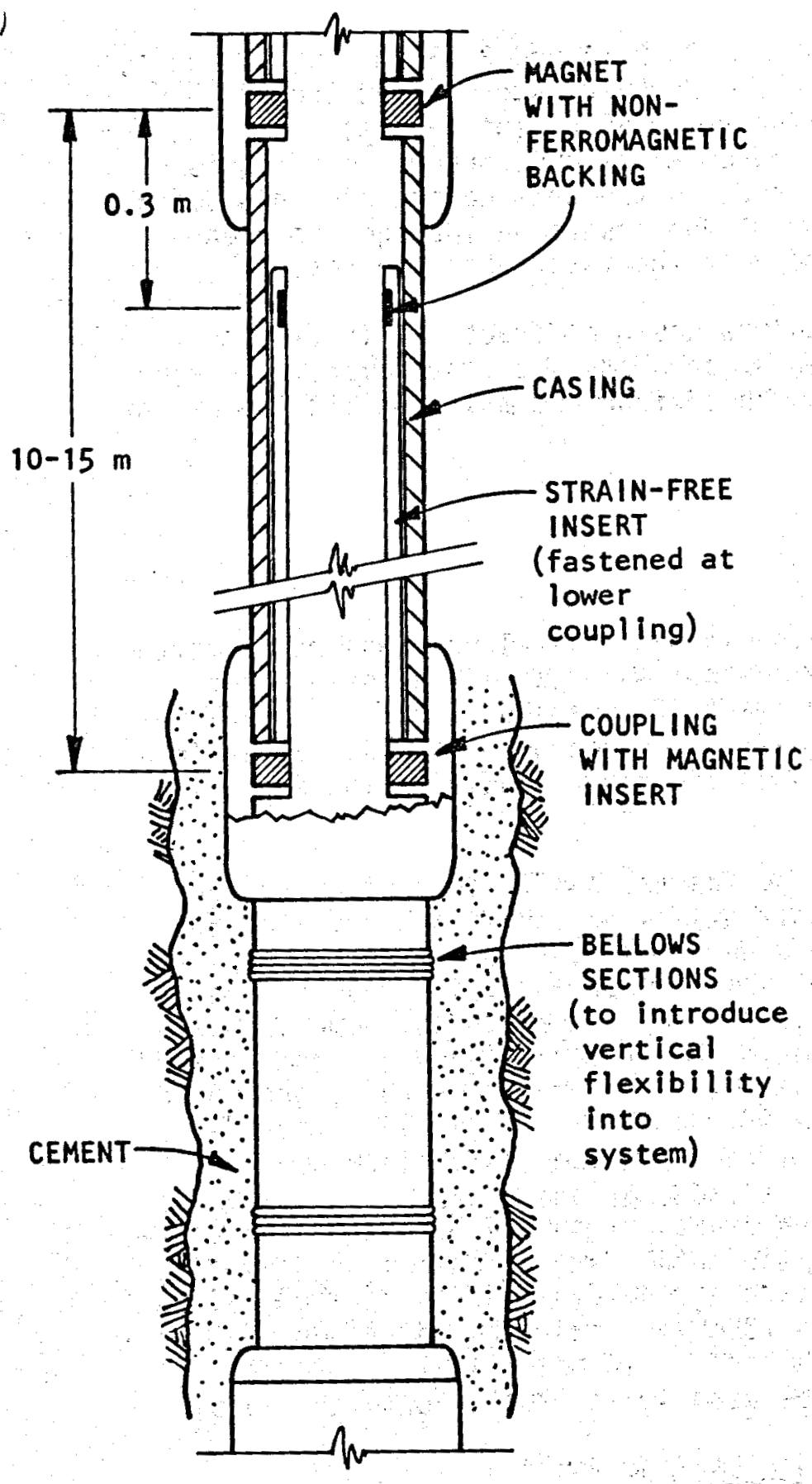

(a) STRAIN-FREE INSERT IN PRODUCTION CASING

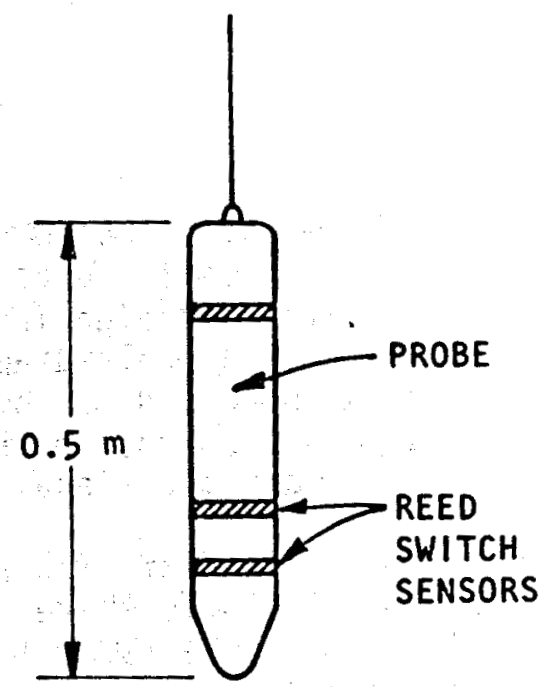

(b) PROBE FOR MONITORING CASING WITH STRAINFREE INSERTS
NOTES:

1) The probe has three sensors. From the strip chart record and from the known sensor spacing on the probe one can calculate the distance between the top of the insert and the coupling (See Figure 2.4 in main text).

2) The probe velocity can be also determined as the probe passes the strain-free insert. This can be used to check the distances calculated from the known lengths on the probe body. 
with instruments proposed in Figures $2.13,2.14$ and 2.15 in the main text, the probe is small and easy to handle. However, strain-free inserts may be difficult to design for the geothemal environment for use in a flowing well. Where the hole diameter increases above each insert, scaling is likely to occur and may bond the insert to the well casing and thus defeat its purpose. Corrosion and erosion of the insert may damage its clean edges and make the signal at the edge of the insert hard to detect.

This application of strain-free inserts could be practical in flowing water or oil wells where environmental problems are less severe. However, in flowing geothermal wells, the harsh environment may prohibit the use of this monitoring technique.

\subsection{MATERIAL PROBLEMS}

\subsection{Super-Invar}

A relatively new controlled expansion alloy, Super-Invar, was not covered in Interim Report 3. Of available controlled-expansion alloys, SuperInvar has the lowest thermal coefficient of expansion: $3.6 \times 10-70 \mathrm{C}$ or about one fourth that of Invar-36 over the range $0^{\circ}$ to $100^{\circ} \mathrm{C}$. Super Invar is available from Burleigh Instruments and their specification sheet is attached as Table 6.1.

For Super-Invar (Burleigh, 1979), the thermal coefficient of expansion increases rapidly above $200^{\circ} \mathrm{C}$. Unit expansion curves are not available for Super-Invar as currently formulated and heat-treated, but an approximate curve is given in Figure 6.1. Where Super-Invar is used in field instrumentation over the range of geothermal temperatures, some problems may result. Length corrections due to temperature change are complicated by the non-linear relationship between temperature and strain, and where accurate knowledge of length change is important, it may be better to select $42 \%$ nickel al loy with a constant expansion coefficient over $0-300^{\circ} \mathrm{C}$. Generally, the reason for selecting Super-Invar is to minimize the total length change due to temperature. From the limited data available (Figure 6.1), it appears that the total length changes of bars of Super-Invar and Low-Expansion "42" are essentially identical when raised from $0^{\circ} \mathrm{C}$ to geothemal temperatures $\left(300^{\circ}\right)$. Thus, the advantage of SuperInvar is lost over this temperature range. An additional limitation of Super-Invar is that it should not be used below $-20^{\circ} \mathrm{C}$ (Burleigh, 1979).

\subsection{High Temperature Electronics and Logging Tools}

Current research in high temperature electrical and mechanical components for geothermal use and testing of prototype logging tools may provide techniques for construction of a geothermal compaction monitoring tool. While these topics were reviewed in depth in Interim Reports 2 and 3, some recent developments are summarized below. 
TABLE 6.1

SUPER-INVAR DATA SHEET

Super-Invar is a special composition of a nickel/iron alloy with an extremely low thermal expansion coefficient: $\alpha \leq 0.36 \times 10^{-6} /{ }^{\circ} \mathrm{C}$. The machinability of Super-Invar makes it ideal for many applications requiring thermal stability. Burleigh offers standard size rounds, bars and flats rough cut to a tolerance of $\pm .2 \mathrm{~cm}$ with a mill quality surface finish. Maximum length of any piece is $180 \mathrm{~cm}$ due to chamber limitations in the Super-Invar heat treating process.

TYPICAL SPECS OF SUPER-INVAR

Thermal Expansion Coefficient at $20^{\circ} \mathrm{C}\left(\mathrm{cm} / \mathrm{cm} /{ }^{\circ} \mathrm{C}\right): \leq 3.6 \times 10^{-7}$

Density (1bs/cu.in.): .294

Tensile Strength (p.s.i.): 69,800

Yield Strenght (p.s.i.): 43,900

Elongation in 2 in., \%: 38

Modulus of Elasticity (p.s.i. $\left.\times 10^{6}\right): 21.5$

Poisson's Ratio: .234

Rockwell Hardness: $R_{b} 77$

MACHINING

The machining of Super-Invar is only slightly more complicated than regular invar. Machining properties are similar to stainless steels. However, slower speeds will be required. An experienced machinist is necessary to achieve high quality surface finishes without cold working the material. Excessive heat generated during the machining process will anneal the SuperInvar and alter the thermal expansion coefficient. (Burleigh, 1979) 


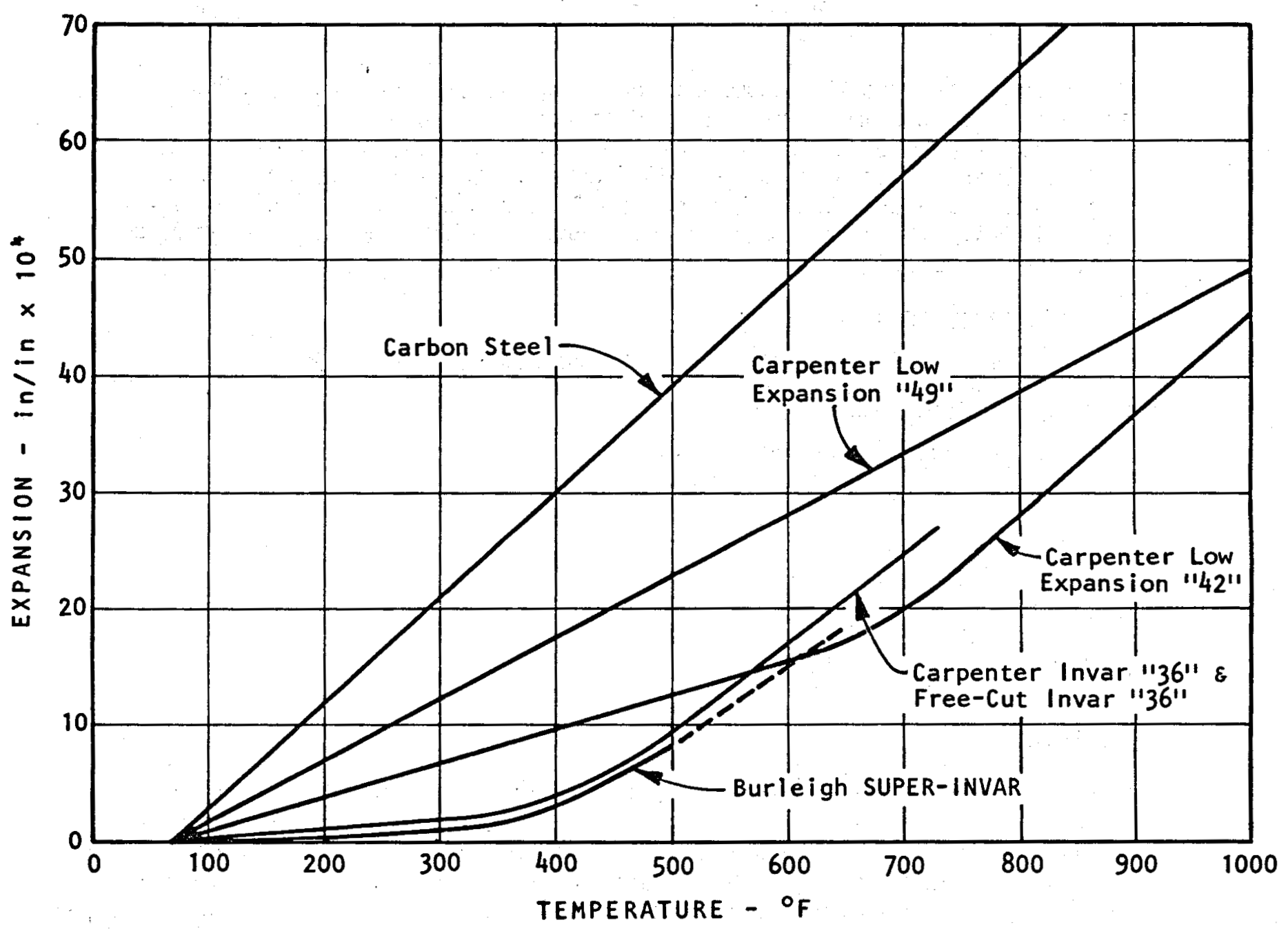

NOTE:

Curve representing SUPER-INVAR is an approximation and is based on a material with the same formulation but a different heat treatment (Burleigh, 1979). Dashed line is our extrapolation.

Figure 6.1 - EXPANSION CURVES FOR LOW EXPANSION ALLOYS 
6.2.1 Basic Circuit Elements - In Table 2.20 and Section 2.3.2 (in main text) we summarized some of the on-going research in high-temperature electronics. Significant progress has been made and reported in the literature since that information was collected for Interim Report No. 3 in early 1978. In view of the rapidly changing state-of-the-art, we present here sources of information rather than a summary of current progress.

An excellent review (Dec. 1978) of high temperature devices, both active and passive, attachment and packaging techniques and circuit fabrication technology can be found in Palmer and Heckman (1978). Sandia Laboratories is a center of this research, and conducts in-house studies and manages outside contracts for DOE in high temperature electronics for its Geothermal Well Technology Logging Instrumentation Program. Further information on their programs can De obtained from Dr. A.F. Veneruso at (505) 264-9162. Another source of high temperature research results is the IEEE Transactions. At the IEEE Midcon Convention in Dallas (Dec. 1978), papers on high temperature electronics were presented by the Naval Research Lab, Harris Semiconductor, Sandia Labs, Micropac, Inc. and Seismic Engineering, Inc.

The rapidly growing knowledge in this area offers promise that suitable electronics and packaging will be available for a geothermal compaction logging tool within the near future.

6.2.2 Logging Tool Development - Development of basic circuit el ements, di scussed above, is the first step in the development of high temperature logging tools. These basic elements have been used to build more complex circuit components, including voltage regulators, line drivers, voltage comparators, special purpose amplifiers and multiplexers for high temperature use. Such devices are now being tested for use to $300^{\circ} \mathrm{C}$ (Palmer and Heckman, 1978). Construction and testing of complete logging tools is now feasible. In a recent test (Sandia Labs, 1979), a temperature logging tool operated successfully for $1-1 / 2$ hours at $275^{\circ} \mathrm{C}$ and for 18 hours at $241{ }^{\circ} \mathrm{C}$ in a geothermal well. Also tested were a flow tool, a low resolution pressure tool and a casing collar locator. The casing collar locator is an upgraded commercial model and currently is not accurate enough for subsidence logging (A. Veneruso, 1979, personal communication). Also under development are a borehole caliper and a borehole televiewer. A brief description of these tools may be found in Table 6.2. These developments listed above suggest that field-tested components for a subsidence monitoring system may be available within a few years.

6.2.3 Logging Accessories - In Interim Reports 2 and 3 we discussed several accessory components for a logging system which could improve current capabilities. The following paragraphs discuss recent advances in these areas.

Cooling System: DOE sponsored research on a refrigeration system to extend the temperature range of existing logging tools has focused on the development of a miniature reciprocating compressor, with steam as a working fluid. Currently, materials problems with respect to valves and 
TABLE 6.2 - GEOTHERMAL LOGGING TOOLS UNDER DEVELOPMENT

\begin{tabular}{|c|c|}
\hline Temperature & $\begin{array}{l}\text { Design based on platinum-resistance transducer; } \\
\text { Compatible with multi- and mono-conductor cables. }\end{array}$ \\
\hline Pressure & $\begin{array}{l}\text { Design uses high resolution quartz crystal } \\
\text { pressure sensor. }\end{array}$ \\
\hline Flow & $\begin{array}{l}\text { Design is a high-temperature impeller-type } \\
\text { transducer with signal feed-through mechanism. }\end{array}$ \\
\hline Caliper & $\begin{array}{l}\text { Upgrade commercial calipers by replacement of } \\
\text { deficient metals, seals and transducer. Four-arm } \\
\text { caliper to be developed first. }\end{array}$ \\
\hline $\begin{array}{l}\text { Borehole } \\
\text { Televiewer }\end{array}$ & $\begin{array}{l}\text { Uses an experimental acoustic sensor, based on a } \\
\text { lead metaniobate transducer; Upgrade existing } \\
\text { Mobil 0il-patented tool for geothermal use. }\end{array}$ \\
\hline
\end{tabular}

(Veneruso et al, 1978; Sandia Laboratories, 1978b; Wonn, 1978; and Veneruso and Stoller, 1978) 
dynamic seals have slowed this research (A. Veneruso, 1979, personal communication).

Cables: Laboratory tests of two cables provided by Vector Cable Company have shown promising results. Aerospace Research Corporation tests of a 7 conductor-PFA teflon insulated cable and a monoconductor. TFE insulated cable are encouraging. Leakage resistance of the 7 conductor cable dropped from 400 GS $\left(G=10^{9}\right)$ to no less than $132 \mathrm{M} \Omega$ under simulated geothermal well logging conditions but returned to normal af ter the test. The TFE monocable's performance was better even at higher temperatures (Sandia Laboratories, 1978b).

Seals: Metal 0-Rings look promising for seals, as shown in Table 6.3a. Elastomers for dynamic seals, blowout preventers, cable insulation and other geothermal uses are under intensive study. Chevron USA tested many oil field rubbers in flowing steam at $450-475^{\circ} \mathrm{F}$. Their test indicated that all the tested rubbers except Ethylene Propylene (EPR/EPDM) lost their resiliency (Snyder, 1978). Similar conclusions were reached by Hirasuna, et a1. (1977). The applications of Ethylene-Propylene are limited, however, because it will not bond to metal (Snyder, 1978). Some high temperature elastometers are summarized in Table 6.3b. A new elastometer, Kalrez, is promising (but costly) and has been used in field logging tools at the Geysers (Snyder, 1978).

\subsection{REFERENCES}

A comprehensive reference list may be found in the main text of this report. 
TABLE 6.3 - HIGH TEMPERATURE ELASTOMER AND SEALS

(Veneruso and Stoller, 1978)

(a) - HIGH TEMPERATURE SEALS

Type

Elas tomeric 0-Rings

Metal 0-Rings

Wave Rings

Metal Softening $500^{\circ} \mathrm{C}$
Performance

Reuse Permitted Least Dirt Sensitive

Reuse not Recommended Soft Plating Recommended Nick and Scratch Sensitive Minor Joint Motion Tolerated

Reuse Not Recommended Soft Plating Recommended Nick and Scratch Sensitive

(b) - HIGH TEMPERATURE ELASTOMERS

\begin{tabular}{|c|c|c|c|c|}
\hline Trade Name & Type & $\begin{array}{l}\text { Decomposition } \\
\text { Temperature }{ }^{\circ} \mathrm{C} \\
\end{array}$ & Cost $\$ / L B$ & Performance \\
\hline BUNA $N$ & NITRILE & 150 & 6 & $\begin{array}{l}\text { Good Oil Resistance } \\
\text { Poor Resistance to } \mathrm{H}_{2} \mathrm{~S} \\
\text { and Steam }\end{array}$ \\
\hline VITON E- $-C$ & FLUORO ELASTOMER & 290 & 35 & $\begin{array}{l}\text { Fair Resistance to Steam } \\
\text { Poor for } \mathrm{H}_{2} \mathrm{~S}\end{array}$ \\
\hline $\begin{array}{l}\text { VITON G } \\
\text { (Peroxide Cure) }\end{array}$ & FLUORO ELASTOMER & 290 & 40 & $\begin{array}{l}\text { Improved Steam Resistance } \\
\text { Poor for } \mathrm{H}_{2} \mathrm{~S}\end{array}$ \\
\hline KALREZ & $\begin{array}{l}\text { FLUORO ELASTOMER } \\
\text { (Fully Fluorinated) }\end{array}$ & 400 & 2000 & $\begin{array}{l}\text { Best for } \mathrm{H}_{2} \mathrm{~S} \\
\text { Fair for steam }\end{array}$ \\
\hline$E P R^{*}$ & POLYOLEFIN & 250 & 8 & $\begin{array}{l}\text { Excellent Steam Resistance } \\
\mathrm{H}_{2} \mathrm{~S} \text { Unknown }\end{array}$ \\
\hline SILOXANE & SILICONE & 300 & 17 & $\begin{array}{l}\text { Poor Steam and Poor } \mathrm{H}_{2} \mathrm{~S} \\
\text { Resistance }\end{array}$ \\
\hline
\end{tabular}

(Ethylene Propylene Terpolymers include EPR and EPDM) 